Critical behavior of thermal expansion and magnetostriction in the vicinity of the first order transition at the Curie point of $\mathrm{Gd}_{5}\left(\mathrm{Si}_{\mathbf{x}} \mathrm{Ge}_{1-\mathrm{x}}\right)_{4}$

by

\title{
Mangui Han
}

A dissertation submitted to the graduate faculty

in partial fulfillment of the requirements for the degree of

DOCTOR OF PHILOSOPHY

Major: Materials Science and Engineering

Program of Study Committee:

David C. Jiles, Major Professor

John E. Snyder

Bulent Biner

Roger Clive Woods

Gary Tuttle

Iowa State University

Ames, Iowa

2004

Copyright $\odot$ Mangui Han, 2004. All rights reserved. 


\section{Table of Contents}

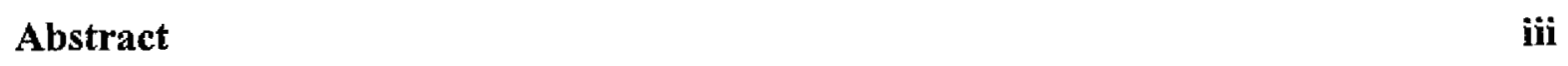

1. Fundamentals of magnetic materials 1

1.1 History of magnetism $\quad 1$

1.2 Magnetic field $\mathrm{H} \quad 2$

1.3 Magnetic induction B 3

1.4 Magnetic moment $m$ and magnetization $M$

1.5 Units Systems in magnetism $\quad 5$

1.6 Demagnetizing field $\quad 6$

1.7 Magnetic materials $\quad 7$

$1.8 \mathrm{M}-\mathrm{H}$ Hysteresis Loop $\quad \mathbf{8}$

1.9 Thermal effect on magnetic materials $\quad 11$

1.10 Anisotropy $\quad 11$

1.11 Domains and domain dynamics $\quad 12$

$\begin{array}{ll}1.12 \text { Curie temperature and Neel temperature } & 18\end{array}$

$\begin{array}{ll}1.13 \text { Critical phenomena in magnetic materials } & \mathbf{2 0}\end{array}$

2. First order and second order transformations $\quad 24$

2.1 Classification of Phase Transformations $\quad 24$

2.2 First Order Transition $\quad \mathbf{2 5}$

2.3 Second Order Transition $\quad 27$

2.4 Characteristics of phase transitions

3. Thermodynamics of the magnetocaloric effect 32

3.1 Components of heat capacity

3.2 Heat capacity, entropy change and temperature differences $\quad 35$

$\begin{array}{ll}3.3 \text { Effect of magnetic field } & \mathbf{3 6}\end{array}$

$\begin{array}{ll}3.4 \text { Magnetocaloric effect } & 38\end{array}$

4. Introduction to the $\mathrm{Gd}_{5}\left(\mathrm{Si}_{\mathrm{x}} \mathrm{Ge}_{1-\mathrm{x}}\right)_{4}$ system 39

5. Thermal expansion and magnetostriction $\quad 44$

5.1 Thermal Expansion $\quad 44$

5.2 Magnetostriction $\quad \mathbf{4 8}$

5.3 Characterization of magnetostriction curve $\quad \mathbf{5 1}$

5.4 Temperature dependence of magnetostriction $\quad \mathbf{5 6}$

$\begin{array}{ll}5.5 \text { RKKY exchange and superexchange coupling } & 57\end{array}$

6. Experimental details $\quad \mathbf{6 0}$

6.1 Closed-cycle refrigerator system

6.2 Thermal expansion $\quad 63$

6.3 Magnetostriction measurements $\quad 66$

$\begin{array}{ll}6.4 \text { Measurement techniques } & 67\end{array}$ 
6.5 Fundamentals of Magnetic Force Microscopy (MFM) $\quad 72$

6.6 Vibrating Sample Magnetometer (VSM)

$\begin{array}{ll}\text { 6.7 Measurement of magnetic field } & \mathbf{7 6}\end{array}$

$\begin{array}{ll}\text { 7. Results and discussions } & \mathbf{7 8}\end{array}$

7.1 $\mathrm{Gd}_{5}\left(\mathrm{Si}_{\mathrm{x}} \mathrm{Ge}_{4-\mathrm{x}}\right)$ with $\mathrm{x}$ range of $0<x \leq 0.3$

$7.2 \mathrm{Gd}_{5}\left(\mathrm{Si}_{x} \mathrm{Ge}_{1-x}\right)_{4}$ with $x$ range of $0.4<x \leq 0.503 \quad \mathbf{8 7}$

$7.3 \mathrm{Gd}_{5}\left(\mathrm{Si}_{\mathrm{x}} \mathrm{Ge}_{4-\mathrm{x}}\right)$ with $\mathrm{x}$ range of $0.575 \leq \mathrm{x} \leq 1$

$\begin{array}{lr}\text { Conclusions } & 148\end{array}$

$\begin{array}{ll}\text { References } & 154\end{array}$

$\begin{array}{lll}\text { Appendix A } & \text { Operation procedures for Janis Closed Cycle refrigerator } & \mathbf{1 5 7}\end{array}$

$\begin{array}{lll}\text { Appendix B Strain gage installation procedure } & 167\end{array}$

$\begin{array}{lll}\text { Appendix C } & \text { Strain gage indicator (model 3800) } & 169\end{array}$

$\begin{array}{ll}\text { Appendix D Operation procedure for VSM measurement } & 171\end{array}$

$\begin{array}{ll}\text { List of publications } & 175\end{array}$

$\begin{array}{ll}\text { Acknowledgements } & 177\end{array}$ 
iii

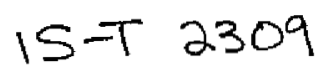

\begin{abstract}
Thermal expansion (TE) and magnetostriction (MS) measurements have been conducted for $\mathrm{Gd}_{5}\left(\mathrm{Si}_{\mathrm{x}} \mathrm{Ge}_{1-\mathrm{x}}\right)_{4}$ with a series of $\mathrm{x}$ values to study its critical behavior in the vicinity of transition temperatures.
\end{abstract}

It was found that the Curie temperature of $\mathrm{Gd}_{5}\left(\mathrm{Si}_{\mathrm{x}} \mathrm{Ge}_{1-\mathrm{x}}\right)_{4}$ for $\mathrm{x} 0 \sim 0.5$ is dependent on magnetic field, direction of change of temperature (Tc on cooling was lower than Tc on heating), purity of Gd starting material, compositions, material preparation methods, and also can be triggered by the external magnetic field with a different $\mathrm{dT} / \mathrm{dB}$ rate for different $\mathrm{x}$ values. For $\mathrm{Gd}_{5}\left(\mathrm{Si}_{1.95} \mathrm{Ge}_{2.05}\right), \mathrm{Gd}_{5}\left(\mathrm{Si}_{2} \mathrm{Ge}_{2}\right), \mathrm{Gd}_{5}\left(\mathrm{Si}_{2.09} \mathrm{Ge}_{1.91}\right)$, it was also found that the transition is a first order magneto-structural transition, which means the magnetic transition and crystalline structure transition occur simultaneously, and completely reversible. Temperature hysteresis and phase coexistence have been found to confirm that it is a first order transformation. While for $\mathrm{Gd}_{5}\left(\mathrm{Si}_{0.15} \mathrm{Ge}_{3.85}\right)$, it is partially reversible at some temperature range between the antiferromagnetic and the ferromagnetic state. For $\mathrm{Gd}_{5}\left(\mathrm{Si}_{2.3} \mathrm{Ge}_{1.7}\right)$ and $\mathrm{Gd}_{5}\left(\mathrm{Si}_{3} \mathrm{Ge}_{1}\right)$, it was a second order transformation between the paramagnetic and ferromagnetic state, because no $\Delta \mathrm{T}$ have been found. Giant magnetostriction was only found on $\mathrm{Gd}_{5}\left(\mathrm{Si}_{1.95} \mathrm{Ge}_{2.05}\right), \mathrm{Gd}_{5}\left(\mathrm{Si}_{2} \mathrm{Ge}_{2}\right), \mathrm{Gd}_{5}\left(\mathrm{Si}_{2.09} \mathrm{Ge}_{1.91}\right)$ in their vicinity of first order transformation. MFM images have also been taken on polycrystal sample $\mathrm{Gd}_{5}\left(\mathrm{Si}_{2,09} \mathrm{Ge}_{1,91}\right)$ to investigate the transformation process. The results also indicates that the Curie temperature was lower and the thermally-induced strain higher in the sample made from lower purity level $\mathrm{Gd}$ starting materials compared with the sample made from high purity Gd metal. 
TE, MS, MFM and VSM measurements on single crystal $\mathrm{Gd}_{5}\left(\mathrm{Si}_{2} \mathrm{Ge}_{2}\right)$ and $\mathrm{Gd}_{5}\left(\mathrm{Si}_{2.3} \mathrm{Ge}_{1.7}\right)$ samples have shown their strong anisotropic behaviors. 


\section{Chapter One}

\section{Fundamentals of Magnetic Materials}

\section{1 History of magnetism}

It is believed that the first magnetic material that was discovered was magnetite $\left(\mathrm{Fe}_{3} \mathrm{O}_{4}\right)$.

More than four thousand years ago, Chinese have already begun to use a lodestone called "Chi Shi" to make a spoon-shape tool (Shi Nan) to tell the direction, shown in Fig.1-1, the handle of spoon is always pointing to the South [1]. According to the book "Meng Xi Bi Tan" written in 1086, a more sensitive compass had been used later in China [1].

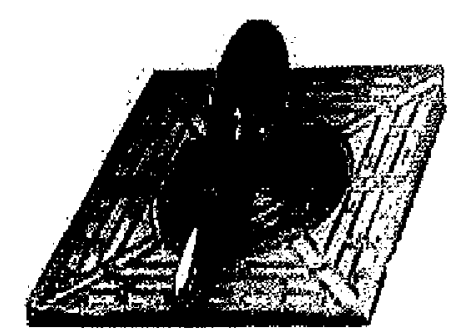

Fig. 1-1 Shi Nan made in China four thousands years ago [1]

Although the interesting phenomenon of ferromagnetic materials has been observed for a long time, the physics behind it was studied since 1600 , a first rational explanation about the mysterious ability of the compass needle to point north-south was given in "De Magnete" written by William Gilbert (1544-1603), a physician in England: "the Earth itself was magnetic" [2]. In the following centuries, when Ampere's Law, Faraday' Law, Maxwell equations, quantum theory of magnetism have been established, magnetism can be scientifically studied on both macroscopical and microscopical scales. 


\subsection{Magnetic field $H$}

There are many sources that can produce magnetic field. Such as, bar magnet, the earth, electromagnet, wire carrying current, etc. However, all of them are related to electric current from the point of macroscopic and microscopic view. In macroscopic view, when a current passes through a length of wire, a magnetic field is generated; the direction of $\mathrm{H}$ can be determined by the so-called "right-hand" rule. If a current carrying wire is formed into a solenoid shape, magnetic field is generated within and around the solenoid. The distribution of $\mathrm{H}$ is shown in Fig.1-2.

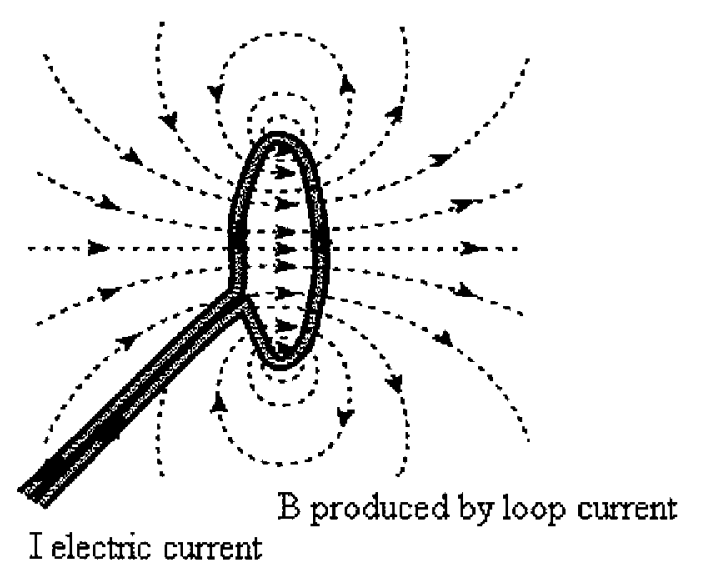

(a)

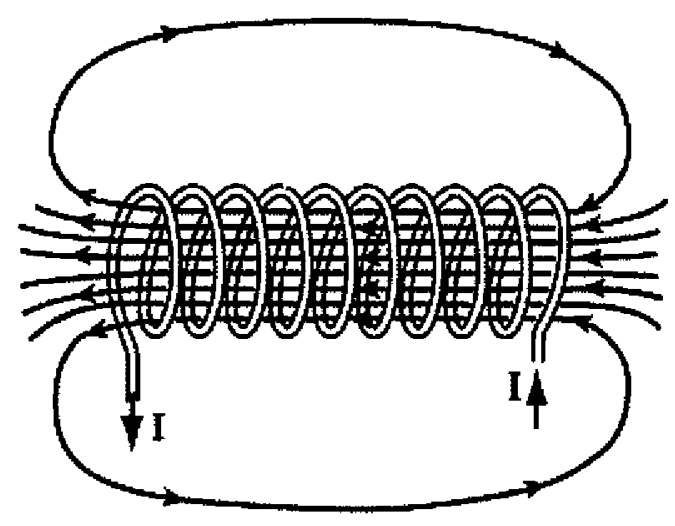

(b)

Fig. 1-2 Magnetic field produced by current in a wire and a solenoid. In (b), $B=\mu_{0} n l$. The pictures are from ref [3]

According to Ampere's Law, the magnetic field at the center of solenoid can be expressed as:

$$
\mathrm{H}=\mathrm{n} \mathbf{I}
$$

Here $\mathrm{n}$ is the number of turns of wire per meter, $\mathrm{l}$ is the current.

Magnetic materials can also produce a magnetic field. From the microscopic point of view, the underlying scientific fact is that there are orbital motions and spins of moving electrons inside atoms. Moving charges produce a so-called "molecular current", a magnetic moment 
results. If the directions of magnetic moments are lined up, a magnetization within the material is formed, and a magnetic field is generated outside.

\subsection{Magnetic induction $B$}

When a magnetic field is present in a medium, such as in free space, magnetic flux $\Phi$ will result. The SI unit of flux is called Weber. According to the following equation, the flux density can be expressed as:

$$
B=\frac{\Phi}{A}
$$

where $A$ is the area that magnetic flux passing through, the flux density in webers per square meter is also called magnetic induction B. A flux density of 1 Weber per square meter is identical to a magnetic induction of 1 tesla. There are two contributing factors for $B$, one is magnetic field in free space. Their relationship can be written as:

$$
B=\mu_{0} H
$$

where $\mu_{0}$ is a universal constant, and is called the permeability of free space. The value of $\mu_{0}$ is equal to $4 \pi \times 10^{-7} \mathrm{H} \mathrm{m}^{-1}$.

When a magnetic field is applied to a magnetic material, the magnetic induction $B$ around the magnetic material will be altered, depending on the types of magnetic materials. If it is a paramagnetic material, $\mathrm{B}$ is a slightly greater than the value of $\mu_{0} \mathrm{H}$. If it is a ferromagnetic material, B can be greatly enhanced by the following equation.

$$
B=\mu_{0}(H+M)
$$


If it is diamagnetic material, $\mathrm{B}$ will be slightly less than the value of $\mu_{0} \mathrm{H}$. From the equation $1-4$, where $\mathrm{B}$ is in Tesla, and $\mathrm{H}$ and $\mathrm{M}$ are in amperes per meter, it is easy to see that there are two factors which contribute to the B value: from the applied magnetic field $\mathrm{H}$, and from the magnetization of material $\mathrm{M}$. The magnetization is the result of net magnetic moment of spin and orbital angular momentum of electrons within the solid and the domain configuration within a magnetic material. Detailed discussions can be found in the references [2]

\subsection{Magnetic moment $m$ and magnetization $M$}

We have mentioned the magnetic moment in solids before. Here we are going to talk in more detail. Magnetic moment, usually its symbol is with a lower case letter $\mathbf{m}$, is the most elementary unit of magnetism. According to reference [4], there are two widely used mathematic approximation models, the equivalent current loop model and the dipole model, as shown in Fig. 1-3. In the equivalent current loop model, the magnitude of the magnetic moment is the product of the current $i$ and the area of the loop $A$. The direction of the magnetic moment is determined by the right-hand rule. Microscopically speaking, the current is due to the motion of electrons and spins of unpaired electrons. However, in the dipole model, the magnitude of the magnetic moment equals the product of the pole strength $p$ and the distance between poles $l$. The direction of the magnetic moment points from the South (p) pole to the North Pole $(+p)$.

For magnetic materials, magnetization $M$ is defined as the vector sum of magnetic moment per unit volume of a solid.

$$
\mathbf{M}=\sum \mathbf{m} / \mathbf{V}
$$

The unit of magnetization $\mathrm{M}$ is $\mathrm{A} / \mathrm{m}$. 

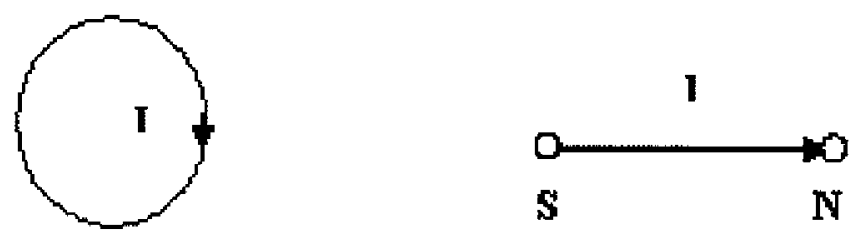

Fig. 1-3 Current loop model and dipole model

In a magnetic field, some magnetic materials will respond to the applied field by changing their magnetization based on the following equation.

$$
\mathbf{M}=\chi \mathbf{H}
$$

Where $\chi$ is called susceptibility. Table 1 gives the susceptibility for some materials.

Table 1 . Susceptibility of some materials $[2,4]$

\begin{tabular}{c|l|c}
\hline Materials & \multicolumn{1}{|c}{ Types } & Susceptibility $(\chi)$ \\
\hline $\mathrm{Al}$ & Paramagnetic & $1.65 \times 10^{-6}$ \\
\hline $\mathrm{Ge}$ & Diamagnetic & $-0.56 \times 10^{-6}$. \\
\hline $\mathrm{Fe}$ & Ferromagnetic & 3000 \\
\hline $\mathrm{MnZn}\left(\mathrm{Fe}_{2} \mathrm{O}_{4}\right)_{2}$ & Ferrimagnetic & 2500 \\
\hline Terbium & Antiferromagnetic & $9.51 \times 10^{-2}$ \\
\hline
\end{tabular}

\subsection{Units Systems in magnetism [4]}

\begin{tabular}{c|c|c|c|c}
\hline Quantity & Symbol & $\begin{array}{c}\text { SI } \\
\text { (Sommerfeld }\end{array}$ & $\begin{array}{c}\text { SI } \\
\text { (Kennelly) }\end{array}$ & $\begin{array}{c}\text { EMU } \\
\text { (Gaussian) }\end{array}$ \\
\hline Magnetic field & $\mathbf{H}$ & $\mathrm{A} \mathrm{m}^{-1}$ & $\mathrm{~A} \mathrm{~m}^{-1}$ & Oersteds \\
\hline magnetic flux & $\boldsymbol{\phi}$ & Weber & Weber & Maxwell \\
\hline Magnetic Induction & $\mathbf{B}$ & Tesla & Tesla & Gauss \\
\hline Intensity of magnetization & $\mathbf{I}$ & $\cdots$ & Tesla & --- \\
\hline
\end{tabular}




\begin{tabular}{|c|c|c|c|c|}
\hline Quantity & Symbol & $\begin{array}{c}\text { SI } \\
\text { (Sommerfeld }\end{array}$ & $\begin{array}{c}\text { SI } \\
\text { (Kennelly) }\end{array}$ & $\begin{array}{c}\text { EMU } \\
\text { (Gaussian) }\end{array}$ \\
\hline Moment & $\mathbf{m}$ & $\mathrm{Am}^{2}$ & Weber meter & Emu \\
\hline Pole Strength & $\mathbf{p}$ & $\mathrm{Am}$ & Weber & $\mathrm{emu} \mathrm{cm} \mathrm{cm}^{-1}$ \\
\hline $\begin{array}{l}\text { Energy of moment } \\
\text { (in free space) }\end{array}$ & & $E=-\mu_{0} \mathbf{m} \cdot \mathbf{H}$ & $E=-\mathbf{m} \cdot \mathbf{H}$ & $E=-m \cdot H$ \\
\hline $\begin{array}{l}\text { Torque on moment } \\
\text { (in free space) }\end{array}$ & & $\tau=\mu_{0} \mathbf{m} \times \mathbf{H}$ & $\tau=\mathbf{m} \times \mathbf{H}$ & $\tau=\mathbf{m} \times \mathbf{H}$ \\
\hline Field Equation & & $\mathbf{B}=\mu_{0}(\mathbf{H}+\mathbf{M})$ & $\mathbf{B}=\mu_{0} \mathbf{H}+\mathbf{I}$ & $\mathrm{B}=\mathrm{H}+4 \pi \mathrm{M}$ \\
\hline
\end{tabular}

Conversion factors:

1 Oersted $=79.58 \mathrm{~A} \mathrm{~m}^{-1}$

1 Gauss $=10^{-4} \mathrm{~T}$

$1 \mathrm{emu} \mathrm{cm}^{-3}=1000 \mathrm{~A} \mathrm{~m}^{-1}$

1 Weber $=10^{8}$ maxwells

$1 \mathrm{emu} / \mathrm{cm}^{3}=12.57 \times 10^{-4}$ weber $/$ meter $^{2}$

$1 \mathrm{erg} / \mathrm{cm}^{3}=10^{-1} \mathrm{~J} / \mathrm{m}^{3}$

$1 \mathrm{emu} / \mathrm{g}=1$ A. $\mathrm{m}^{2} / \mathrm{kg}=4 \pi \times 10^{-7} \mathrm{~Wb} \cdot \mathrm{m} / \mathrm{kg}$

\subsection{Demagnetizing field}

If we consider a magnetized material with a finite dimension using the dipole model, both ends of the material should be two magnetic poles, which themselves cause a magnetic field in the material. The direction of this field is opposite to the direction of the magnetization. The field is then called the demagnetizing field $\mathbf{H}_{\mathrm{d}}$. The demagnetizing field depends on the magnetization of the material and demagnetizing factor, $\mathrm{N}_{\mathrm{d}}$, which is determined by the sample geometry.

$$
\mathbf{H}_{\mathrm{d}}=-\mathrm{N}_{\mathrm{d}} \mathbf{M}
$$


The demagnetizing field plays an important role in magnetic materials, particularly in high permeability materials $(\mathbf{M}>>\mathbf{H})$ and permanent magnets. Approximate calculated values of $\mathrm{N}_{d}$ can be found by using a chart. The following figure shows such a chart.

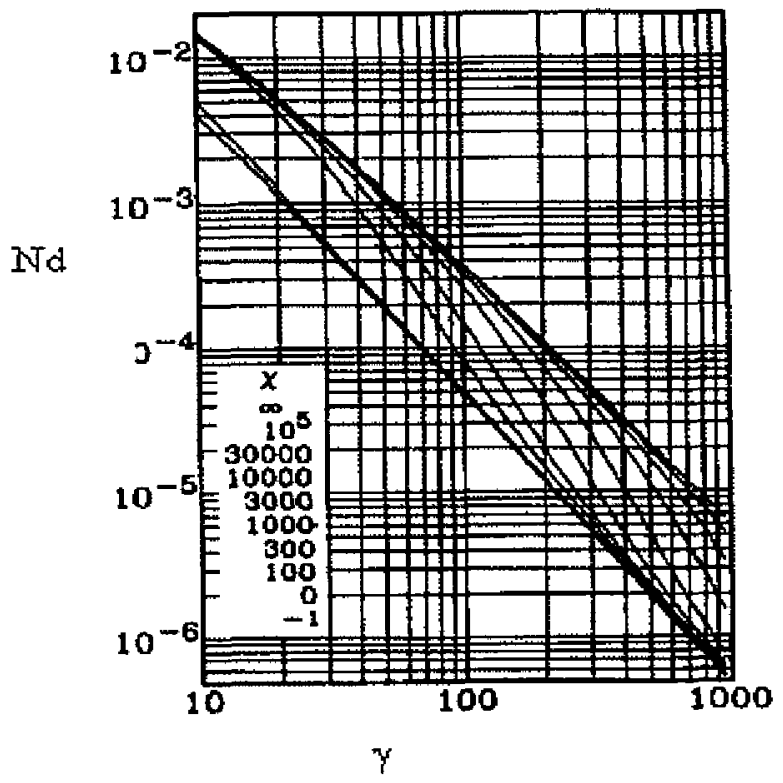

Fig. 1-4 Demagnetization factor $N_{d}$ for a cylinder with the field applied along the unique axis of the cylinder, where $\gamma$ is the ratio of length to diameter [5]. The different curves represent different values of susceptibility $\chi$.

\subsection{Magnetic materials}

Traditionally, magnetic materials are classified into three groups: diamagnets, paramagnets, and the group of ferromagnets, antiferromagnets, and ferrimagnets; according to their responses to a magnetic field, such as susceptibility $\chi$. Diamagnets are those materials for which susceptibilities are negative and it is about $-10^{-5}$. When an external magnetic field is exerted on it, their magnetic moments align along the opposite direction to the external magnetic field. Paramagnets are those materials for, which $\chi$ are positive and equal to about $10^{-3}-10^{-5}$, which means it needs a very strong $\mathrm{H}$ so that magnetic moments can completely 
align along the direction of $\mathrm{H}$. The third group is called ferromagnetic material. Their susceptibility is positive and typically has value $50-10,000$, which means a relatively small field can align magnetic moments along the direction of applied field. Antiferromagnetic material has strong exchange like a ferromagnet, but its susceptibilities are small like paramagnet, because antiferromangets usually have two sublattices whose magnetic moments are aligned antiparallel. Ferrimagnetic materials, such as $\mathrm{Fe}_{3} \mathrm{O}_{4}$, are a special kind of antiferromagnetic material, which usually have two sublattices. Magnetic moments in each lattice are aligned antiferromagnetically, but with different magnitudes. The result is that the net magnetization is not zero. Ferrimagnetic ferrite materials have very good high frequency properties.

\subsection{M-H Hysteresis Loop}

The response of magnetization to the cyclic magnetic field $\mathrm{H}$ can be recorded by a loop called the $\mathrm{M}-\mathrm{H}$ hysteresis loop, which is shown in Fig. 1-5. The $\mathrm{x}$ axis is the magnetic field $\mathrm{H}$, the $y$ axis is magnetization $M$. The possible causes for hysteresis which include: (1) imperfections in materials such as impurity elements, dislocations which will cause the magnetization process to require more energy to overcome the internal friction. (2) Magnetocrystalline anisotropy is another mechanism which can cause the hysteresis. For instance, ferromagnetic materials with high anisotropy show greater hysteresis. Detailed discussion of magnetocrystalline anisotropy will be given later.

Starting from the demagnetization state, point $\mathrm{O}, \mathrm{M}$ increases with $\mathrm{H}$ until it saturates at point $B$, where $M$ is called saturation magnetization. In the first section of $O A, M$ increases 
with $\mathrm{H}$ approximately linearly. Generally speaking, this process is due to the reversible domain wall motion, such as domain bending at low field magnitude. In the section $\mathrm{AB}, \mathrm{M}-\mathrm{H}$ relationship is nonlinear, it is due to the irreversible domain wall motion (such as domain walls passing through pinning sites), and irreversible magnetic moments rotation from its original easy axis to the easy axis closest to the direction of $\mathrm{H}$. When $\mathrm{H}$ decreases to zero, $\mathrm{M}$ does not return to zero, and at point $C$, it is called remanent magnetization $M_{R}$. The negative field $\mathrm{H}$ needed to reduce $\mathrm{M}$ to zero at second quadrant is called intrinsic coercivity ${ }_{M} \mathrm{H}_{c}$, point $\mathrm{D}$ in the curve. If the magnetic field magnitude is increased further, the ferromagnetic material will saturate at point $\mathrm{E}$. If $\mathrm{H}$ is removed, it will go back to point $\mathrm{G}$.

As seen from the $\mathrm{M}-\mathrm{H}$ hysteresis loop, $\mathrm{M}$ value depends on the magnetizing history. There are two methods that can demagnetize the material, back to point $\mathrm{O}$. One way is to heat the material above its Curie temperature and then cool down. The other way is to apply a magnetic field with constant frequency and decreasing field magnitude, as shown in Fig. 1-6.

According to the coercivity value, magnetic materials can be simply classified into two groups: soft magnets and hard magnets (also sometimes called permanent magnets). Generally speaking, magnetic materials with coercivities greater than $10 \mathrm{kA} / \mathrm{m}$ are hard magnets and soft magnets are those with coercivities below $1 \mathrm{kA} / \mathrm{m}$. 


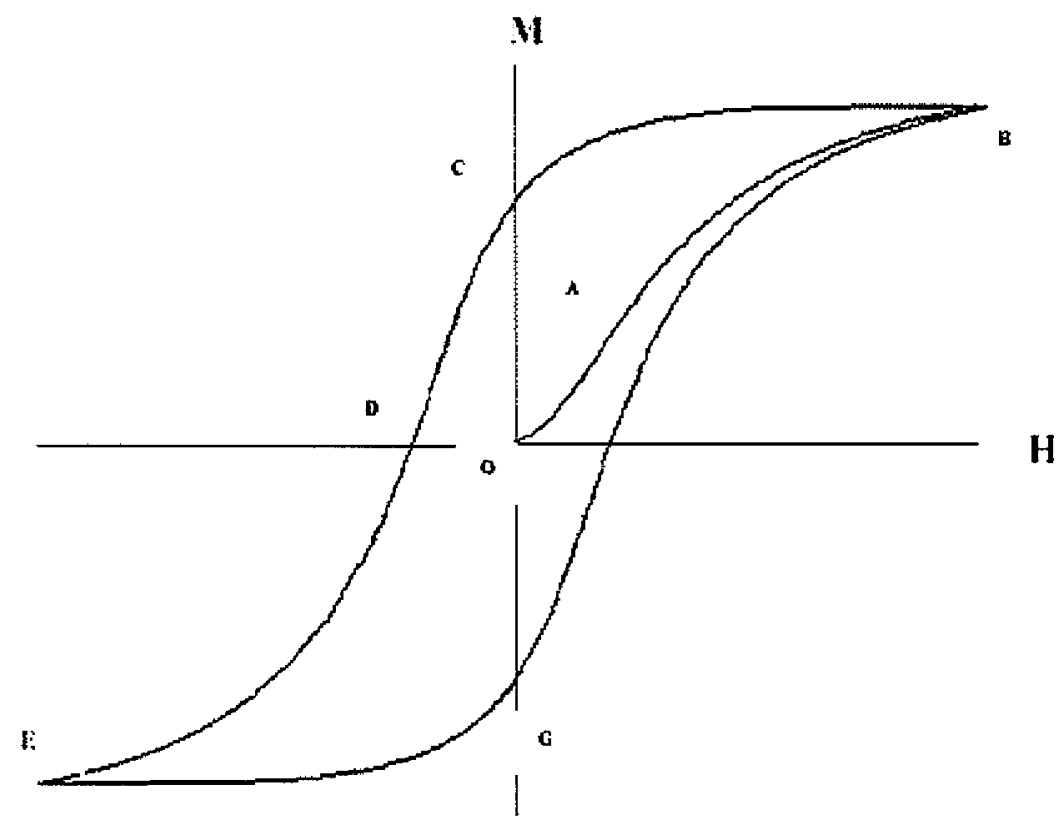

Fig. 1-5 M-H hysteresis loop.

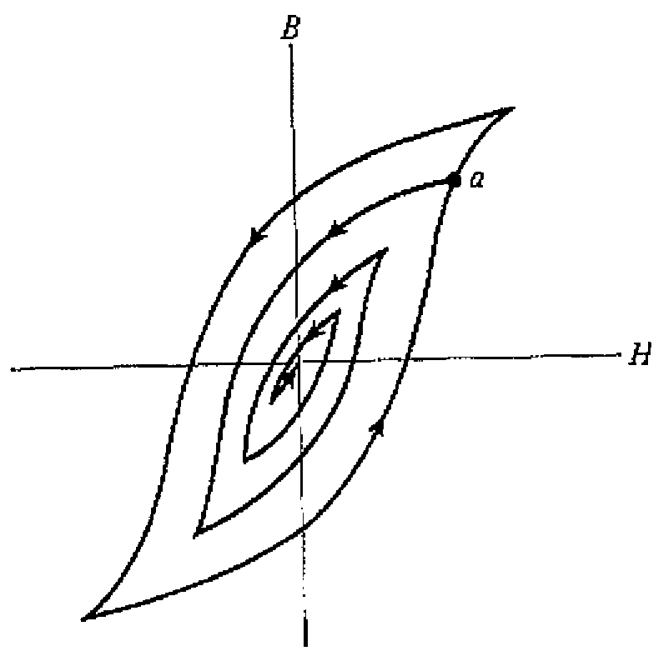

Fig. 1-6 Demagnetizing process by cycling $H$ with decreasing amplitude. Picture is from ref [2] 


\subsection{Thermal effect on magnetic materials}

All ferromagnetic materials have maximum spontaneous magnetization within the domains at $0 \mathrm{~K}$ and magnetization decreases as the temperature increases. The decrease of spontaneous magnetization is due to magnetic moment precession relaxation caused by thermal energy. Furthermore, the materials become paramagnetic when heated to a characteristic temperature, which is called the Curie temperature, $T_{\mathfrak{c}}$. At the $T_{\mathfrak{c}}$, the spontaneous magnetization of the material suddenly drops. The reason is that the thermal energy of the magnetic moment reaches a critical value at the Curie temperature which destroys the magnetic order in the ferromagnetic materials.

\subsection{Anisotropy}

In some degree, all magnetic materials are anisotropic, which implies that the magnetic properties are not completely identical in all directions. For example, in terms of $\mathrm{M}-\mathrm{H}$ curves, it has been found that magnetic field required to saturate magnetization are different when measurements are conducted along different directions. The preferred directions of magnetization are called magnetic easy axes. This can be explained by domain rotation mechanism against the energy of crystal anisotropy. The anisotropic energy is defined as the value of energy required to rotate the magnetization from a preferred "easy" direction into a so-called "hard" direction. For a cubic crystal, such as iron single crystal, and supposing the $M_{s}$ makes angles $\theta_{1}, \theta_{2}, \theta_{3}$ with the three principal crystal directions.

$$
E_{a}=K_{0}+K_{1}\left(\cos ^{2} \theta_{1} \cos ^{2} \theta_{2}+\cos ^{2} \theta_{2} \cos ^{2} \theta_{3}+\cos ^{2} \theta_{1} \cos ^{2} \theta_{3}\right)+K_{2}\left(\cos ^{2} \theta_{1} \cos ^{2} \theta_{2} \cos ^{2} \theta_{3}\right)+\ldots
$$


A one-constant equation is used as a first approximation of the anisotropy of cubic materials [2]

$$
\mathrm{E}_{\mathrm{a}}=\mathrm{K}_{1}\left(\cos ^{2} \theta_{1} \cos ^{2} \theta_{2}+\cos ^{2} \theta_{2} \cos ^{2} \theta_{3}+\cos ^{2} \theta_{1} \cos ^{2} \theta_{3}\right)
$$

For a hexagonal crystal, such as a cobalt single crystal, the anisotropy energy can be represented by a uniaxial approximation [2]

$$
E_{a}=K_{0}+K_{u 1} \sin ^{2} \phi+K_{u 2} \sin ^{4} \phi+\ldots
$$

where $\phi$ is the angle of magnetization with respect to the unique axis. Using a one constant approximation, it can be simplified as axial anisotropy by the equation:

$$
E_{a}=K_{u 1} \sin ^{2} \theta
$$

In the following table, anisotropy constants have been given for some substances.

Table 2. Anisotropy Coefficients

\begin{tabular}{c|c|c|c}
\hline Structure & Substance & $\mathrm{K}_{1}\left(10^{4} \mathrm{~J} / \mathrm{m}^{3}\right)$ & $\mathrm{K}_{2}\left(10^{4} \mathrm{~J} / \mathrm{m}^{3}\right)$ \\
\hline \multirow{3}{*}{ Cubic } & $\mathrm{Fe}$ & 4.8 & \pm 0.5 \\
\cline { 2 - 4 } & $\mathrm{Ni}$ & -0.5 & -0.2 \\
\hline \multirow{3}{*}{ Hexagonal } & $\mathrm{Co}$ & 45 & 15 \\
\cline { 2 - 4 } & $\mathrm{MnBi}$ & 89 & 27 \\
\hline
\end{tabular}

\subsection{Domains and domain dynamics}

(1) Domains and domain walls

The formation of domains and domain walls can be explained by thermodynamic energy minimization in magnetic materials. The magnetostatic energy (MS energy for short) and domain wall energy (DW energy for short) play critical roles in the configuration of domains. 
The domain size is the result of competition between the magnetostatic energy and the domain wall energy. The magnetostatic energy tends to decrease the size of domains while the domain wall energy tends to increase the size. Typically, there are $10^{12}$ to $10^{18}$ atomic moments in each domain [4]. Magnetostatic energy is expressed by the following equation.

$$
E=-\mu_{0} \int H_{d} \cdot d M=\mu_{0} N_{d} \int M \cdot d M=\mu_{0} N_{d} M^{2} / 2
$$

The magnetostatic energy tends to keep the net bulk magnetization zero so that the energy can be minimized. If the demagnetization process starts from the saturated condition, originally, the sample consists of a single domain. The single domain breaks into several small regions to provide a flux closure at the ends of specimen. The result is that the magnetostatic energy is reduced. Within each domain, the magnetic moments are aligned parallel. In fully demagnetized states, the orientations of domains are random if the materials are isotropic and are along their crystallographic magnetic easy axes if the materials are anisotropic. The following figure illustrates in principle how demagnetization proceeds from the saturated state with a single domain to the fully demagnetized state with many domains.

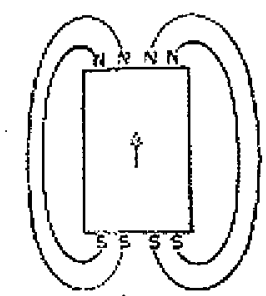

(A)

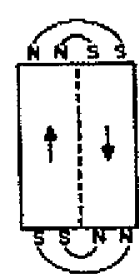

(B)

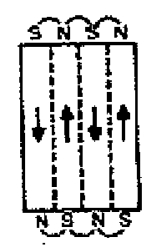

(c)

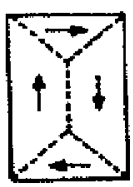

(D)

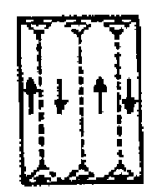

(E)

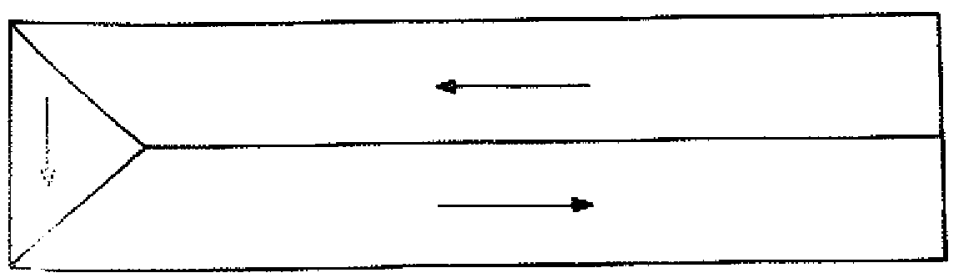

Fig. 1-7 in upper diagram, (A) single domain with higher MS energy; (B) lower MS energy and smaller domain wall energy; (C) much lower MS energy and higher DW energy; (D) and 
(E) lower MS energy and higher DW energy. In lower diagram, a closure domain is shown at the end of iron sample. (After Jiles in ref [4])

The region between the adjacent domains is called domain wall, where the magnetic moments gradually change directions from one domain to the other domain, the most common type of domain wall is the Bloch wall which is shown in the following figure.

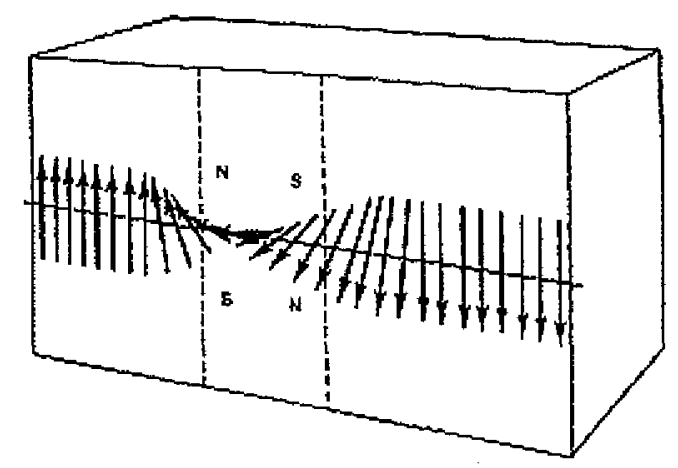

Fig. 1-8 Domain wall (Bloch wall) (after Kittel [6])

There is another type domain wall, called Neel wall, which is usually found in magnetic thin films. In the Neel wall, the moments rotate within the plane of the specimen, as shown below.

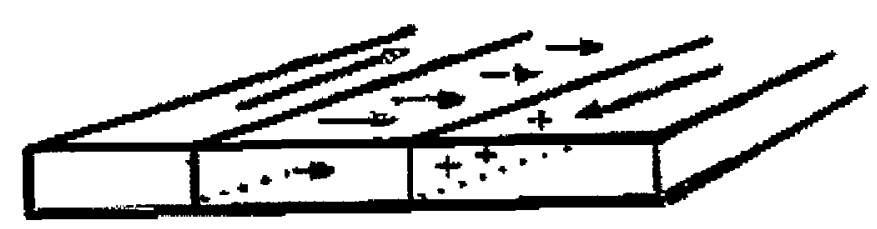

Fig. 1-9 Neel wall in thin film [4].

The domain wall thickness is determined by two energies, exchange energy between neighboring moments and anisotropy energy. The exchange energy tends to keep the angle between moments or neighboring atoms small because the exchange energy is smaller when neighboring moments are aligned closer to parallel. The anisotropy, on the other hand, tends to make the wall thinner since the anisotropy energy is smaller when all moments align along 
easy axes. The domain wall energy is considered as the sum of exchange energy and anisotropy energy of the moments in a unit area of domain wall. The domain wall energy per unit area of the wall can be written as [4]:

$$
\gamma=\frac{\mu_{0} \xi m^{2} \pi^{2}}{\delta a}+K_{\mathrm{I}} \delta
$$

where $a$ is the lattice spacing, $K_{1}$ is the anisotropy constant, and $m$ is magnetic moment of atoms. The first term on the right of above equation is the exchange energy; the second term is the anisotropy energy.

The domain wall width is determined by minimizing the domain wall energy with respect to its width, therefore,

$$
\frac{d \gamma}{d \delta}=\frac{-\mu_{0} \xi m^{2} \pi^{2}}{\delta^{2} a}+K_{\mathrm{l}}=0
$$

Then the domain wall width $\delta$ can be calculated as [4]:

$$
\delta=\sqrt{\frac{\mu_{0} \xi m^{2} \pi^{2}}{K_{1} a}}
$$

(2) Domain wall pinning

The domain and domain wall structures in magnetic materials not only depend on the material's composition, which determines the exchange energy and the domain wall energy, but also depends on the metallurgy and treatment of the materials, which determines distribution of stress, dislocations, voids and second phases. Since these defects result in local energy minima when domain walls intersect them, an extra energy is needed for domain walls to move. This is called the domain wall pinning energy. In other words, it is the magnetoelastic coupling between the structural defects and the domain walls that affects the 
motion of the domain wall, therefore controls the magnetic properties, such as permeability and coercivity.

\section{(3) Domain processes}

In the demagnetized state, magnetic domains are randomly, arranged while magnetic moments within domains are all aligned collinear to each other along one of the crystallographic easy axes. Under the action of an applied magnetic field, magnetic domain processes occur. The driving force comes from the magnetic field energy, which is the energy of individual magnetic moment $\boldsymbol{m}$ in a magnetic field $\mathrm{H}$.

$$
E=-\mu_{0} \vec{M} \cdot \vec{H}
$$

which ensures that the energy minimum occurs when all moments are aligned parallel with the applied field. Basically, there are two domain processes: domain wall motion and domain rotation. The domain wall motion process causes the growth of domains which are aligned with respect to the field together with a reduction in size of domains which are aligned in other directions. The domain rotation process occurs when the domains which are not in the direction of the applied magnetic field, overcome the anisotropy energy and rotate towards the direction of the field. Both processes can be either reversible or irreversible. In fact, the domain wall motion is also caused by localized rotation of magnetic moments, where the rotating moments are located within the domain walls and each moment rotates through a small angle.

Generally, the number of moments within domain walls is much less than the number of moments within domains. At low applied field, domain wall motion usually dominates the 
magnetization processes unless there are very strong pinning sites when domain wall energies are too high to be moved, e.g. very hard materials. Wall motion incorporates two distinct effects: domain wall bowing and translation. Domain wall bowing occurs when the domain wall energy is low and the pinning strength is strong. Domain wall bowing is a reversible process at low field, in which a domain wall under the action of a magnetic field behaves like an elastic membrane. When the field is removed the wall returns to its original position. Domain wall bowing becomes irreversible once the domain wall is sufficiently deformed that the expansion continues without further increase of field. Wall translation is usually irreversible and occurs at moderate field strength unless the material is nearly free of defects, which implies few domain wall pinning sites. In the domain wall translation process, the domain wall breaks away from its current pinning site and becomes pinned by another pinning site. At higher applied magnetic field, the domain wall bowing and translation processes might occur together.

At moderate field strength, domain rotation becomẹs significant because the magnetic field energy can be high enough to overcome the anisotropy energy. When the angular displacement of the domain's magnetization is slight, it is reversible. When the moments rotate from their original easy axes to the easy axis closest to the field direction, it is irreversible.

At some point, all moments are aligned along their closest easy axes within all crystalline grains. In other words, single domains are formed at high fields in all single grains in a polycrystalline material and a single domain is obtained in a single crystal material. At yet higher applied field, coherent rotation occurs. The magnetic moments are gradually rotated from preferred easy axes towards the field direction. This process is also reversible. Finally 
at very high fields there is a reversible change in which the magnetic moments within the sample, which is already a single domain, are aligned more closely with the field direction. This occurs because the individual magnetic moments precess about the field direction due to thermal energy. As the field strength increases the angle of precession is reduced.

\subsection{Curie temperature and Neel temperature}

All ferromagnetic materials when heated to sufficiently high temperatures become paramagnetic. The transition temperature from ferromagnetic to paramagnetic behavior is called the Curie temperature. At this temperature the permeability of the material drops suddenly and both coercivity and remanence become zero. If the unpaired electronic magnetic moments which are responsible for the magnetic properties are localized on the atomic sites then we can consider an interaction between the unpaired moments. This interaction, which Weiss introduced to explain the paramagnetic susceptibilities of certain materials, leads to the existence of a critical temperature below which the thermal energy of the electronic moments is insufficient to cause random paramagnetic alignment. This means that the effective field $\mathrm{H}_{\mathrm{eff}}$ can be used to explain the alignment of magnetic moments within domains for temperature below Tc. There are several theories to explain this critical phenomenon: the mean-field approximation, which was used successfully in the paramagnetic region, and a nearest-neighbor-only coupling, which is more appropriate in the ferromagnetic regime. The Curie temperature is expressed as follows on the basis of the mean-field model, assuming that the magnetic moments do not experience any anisotropy.

$$
T_{c}=\frac{\mu_{0} N \alpha m^{2}}{3 k_{B}}
$$


Similarly for a nearest-neighbor coupling, $\mathrm{Tc}$ is given as below.

$$
T_{c}=\frac{\mu_{0} z \xi m^{2}}{3 k_{B}}
$$

The Curie temperature for some magnetic materials is given as below.

Table 3 Curie temperature of some materials, data is from ref [4]

\begin{tabular}{c|c}
\hline Material & Curie temperature ${ }^{0} \mathbf{C}$ ) \\
\hline Iron ( Fe) & 770 \\
\hline Nickel (Ni) & 358 \\
\hline Cobalt (Co) & 1130 \\
\hline Gadolinium (Gd) & 20 \\
\hline Terfenol & $380-430$ \\
\hline $\mathrm{Nd}_{2} \mathrm{Fe}_{14} \mathrm{~B}$ & 312 \\
\hline $\mathrm{Alnico}$ & 850 \\
\hline $\mathrm{Sm}_{2} \mathrm{Co}_{17}$ & 810 \\
\hline
\end{tabular}

When a magnetic material is in paramagnetic regime, its susceptibility $\chi$ obeys the Curie-

Weiss law, which is given by the following equation

$$
\chi=\frac{M}{H}=\frac{C}{T-T_{c}}
$$

As $T$ approaches to the ordering temperature $T c$, the susceptibility $\chi$ becomes very large.

For a simple antiferromagnetic material, the nearest-neighbor moments are aligned antiparallel. According to the Curie-Weiss model, it can be divided into two lattices, A and B. 
Within either $\mathrm{A}$ or $\mathrm{B}$, magnetic moments interact with neighboring moments with a positive coupling coefficient just like ferromagnetic materials. However, the coupling coefficient between $\mathrm{A}$ and $\mathrm{B}$ is negative, which results in neighboring magnetic moments aligning antiparallel. If we plot $1 / \chi$ against temperature for antiferromagnetic material, like one shown below for $\mathrm{Tb}$, a straight line intercepts the temperature axis at a temperature $(230 \mathrm{~K})$ which is called Neel temperature, $\mathrm{T}_{\mathrm{N}}$. Above $\mathrm{T}_{\mathrm{N}}$, it is in paramagnetic disordered state. Below $\mathrm{T}_{\mathrm{N}}$, it is in antiferromagnetic ordered state.

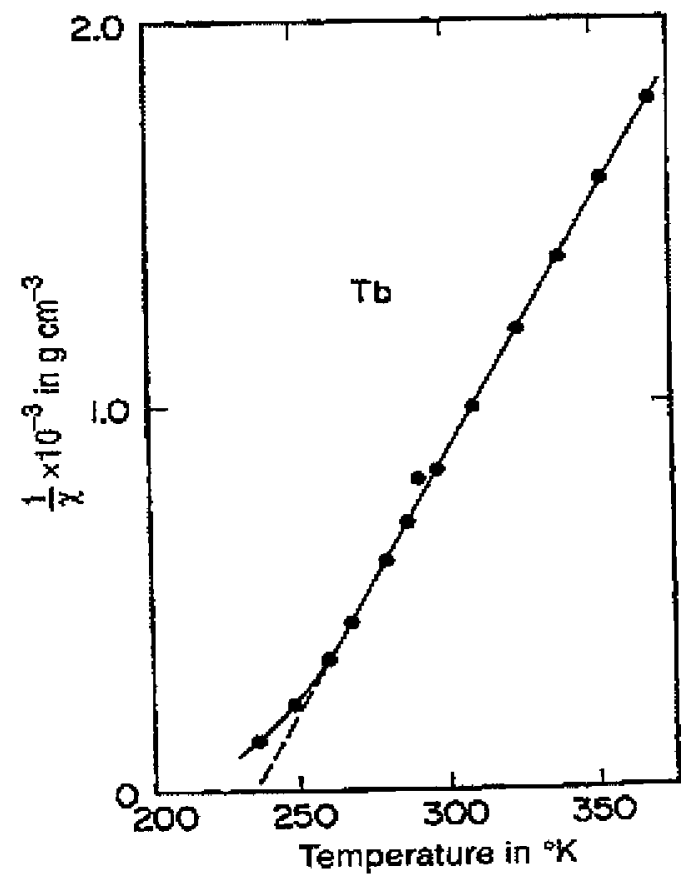

Fig. $1-101 / \chi$ against temperature for $\mathrm{Tb}$ indicating Neel temperature at $\mathrm{T}=230 \mathrm{~K}$, the graph is from ref [7].

\subsection{Critical phenomenon in magnetic materials}

The bulk properties of magnetic materials show anomalous behavior in the vicinity of the transition temperatures such as the Curie points and Neel points. The anomalous behavior is 
due to coupling between the particular property and the magnetic structure, and will be also useful for us to understand these coupling effects. The effects are one of a class of behavior known as critical phenomena. The main objective of this thesis is to study critical behavior for $\mathrm{Gd}_{5}\left(\mathrm{Si}_{\mathrm{x}} \mathrm{Ge}_{1-\mathrm{x}}\right)_{4}$. For a better understanding of the research results in this thesis, we are going to discuss some anomalous behaviors at the ordering temperature here.

\section{(1) Susceptibility anomaly}

As mentioned above when $\mathrm{T}$ approaches to the ordering temperature $\mathrm{Tc}, \chi$ starts to become very large. The variation of $\chi$ with temperature for paramagnetic materials which undergo a transition to ferromagnetism at the Curie temperature $\mathrm{Tc}$ is shown in the figure below.

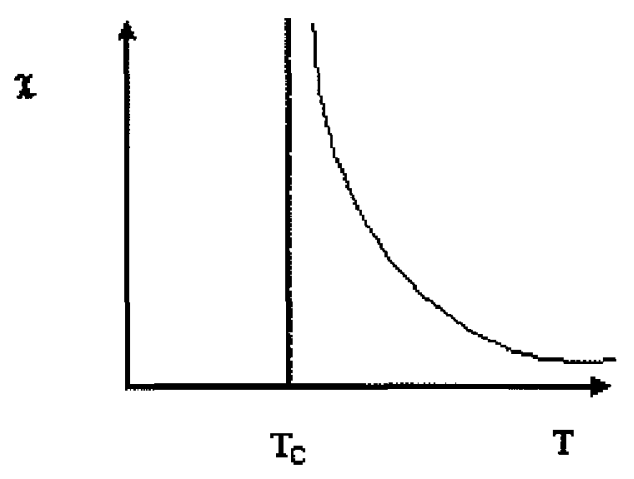

Fig. 1-11 Curie-Weiss Law

\section{(2) Specific heat anomaly}

The specific heat of a ferromagnetic is greater than if the material was a nonferromagnetic and it goes through a maximum at the Tc point. When heat is added to any metal, part of it increases the amplitude of thermal vibration of the ions (lattice specific heat) and the remainder increases the kinetic energy of the valence electrons (electronic specific heat). If the metal is ferromagnetic, then still additional heat is required to disorder the spins 
(magnetic specific heat). The specific heat of a magnetic material has a magnetic component $\mathrm{C}_{\mathrm{m}}$ given by:

$$
C_{m}=\frac{\left(d E_{e x}\right)}{d T}
$$

Where $\mathrm{E}_{\mathrm{ex}}$ is total exchange self-energy of the material per unit volume.

$$
\begin{aligned}
& E_{e x}=-\mu_{0} \alpha M_{s}{ }^{2} \\
& C_{m}=-2 \mu_{0} \alpha M_{s} \frac{d M_{s}}{d T}
\end{aligned}
$$

The number of spins disordered per degree rise in temperature increases with the temperature in accordance with the appropriate Brillouin function, and becomes very large just below the Curie temperature, where the magnetization is decreasing rapidly with temperature. The specific heats of materials which undergo order-disorder transitions show lambda-type anomalies at the critical temperature. The variation of $\mathrm{C}_{\mathrm{m}}$ with temperature is plotted for $\mathrm{Gd}$ in Fig. 1-12 [4].

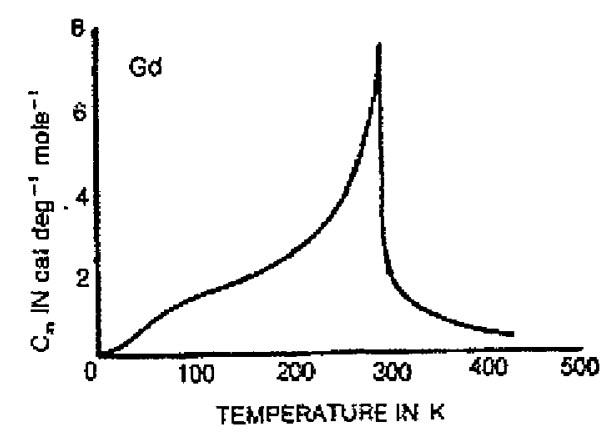

Fig. 1-12 Anomalous heat capacity behavior around the critical temperature [4] 


\section{(3) Elastic constant anomaly}

The elastic constants of materials show critical behavior close to magnetic phase transitions such as the Curie point as shown in Fig. 1-13. Magnetoelastic anomalies are known to occur in rare earth metals as a result of very strong magnetoelastic coupling.

\section{(4) Thermal expansion anomaly}

The thermal expansion and magnetostriction also exhibit unusual behavior at phase transitions such as at the Curie and Neel points will be shown in the later chapter. This is because there is the sudden appearance of spontaneous magnetostriction at the order-disorder transition temperature. This anomaly will be extensively studied in this thesis for $\mathrm{Gd}_{5}\left(\mathrm{Si}_{\mathbf{x}} \mathrm{Ge}_{1-}\right.$ $x)_{4}$.

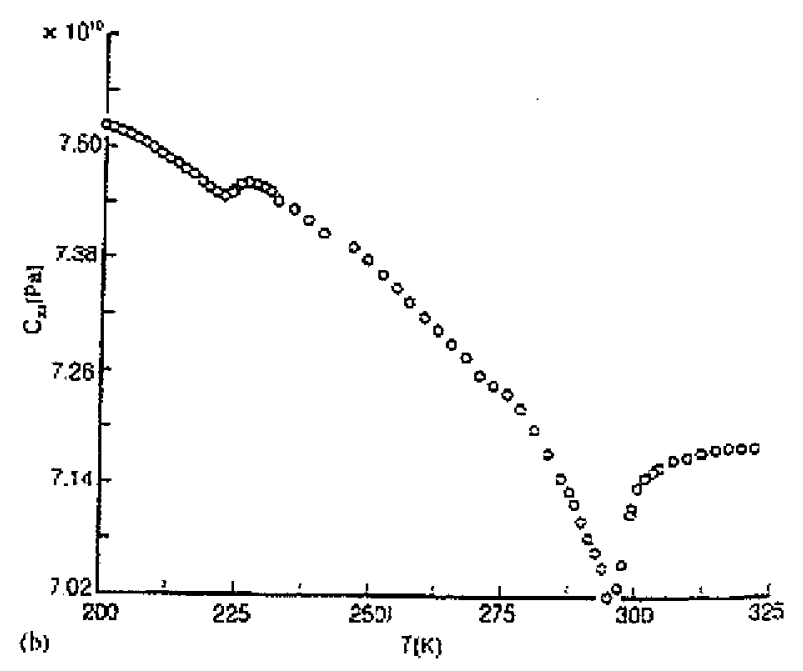

Fig. 1-13 Critical behavior of the elastic constant of gadolinium (Gd) (after Jiles [4]) 


\section{Chapter Two}

\section{First Order and Second Order Transformations}

\subsection{Classification of Phase Transformations}

Phase transformations can be classified from different points of view. In 1933 Ehrenfest devised a classification of phase transitions based on the discontinuity in derivatives of the free energy function [8]. If we use the criterion that whether the phase transformation is completed via atom diffusion or not, the phase transformation can also be classified as diffusion type transformation and non-diffusion transformation. In this thesis, we adopt the Ehrenfest's criterion.

Since the first order and second order transitions are central to the theme of this thesis, it is crucial for us to understand their definitions and their characteristics. If a material transforms from phase $I$ into phase $I I$, its thermodynamic potential $G$ is continuous, but its first derivative (such as entropy S) may have a discontinuity. Another possibility is that the first derivative is continuous, but the second derivative (such as volume thermal expansion coefficient $\alpha$ ) is discontinuous. Therefore, the discontinuity can occur in the first, second derivative or higher derivative of the free energy. Here we give the general classification of phase transformation based on the derivative discontinuity of free energy: If the $n^{\text {th }}$ derivative of free energy is discontinuous, and all lower derivatives are not, the transition is of $n^{\text {th }}$ order. 


\subsection{First Order Transition}

As a matter of fact, most of phase transformations we come across are first order transitions. Here we give an example of first order transition to show its characteristics: the pure metal solidification process. When the pure metal liquid is cooled at very slow rate, the curve of temperature versus time is given as following.

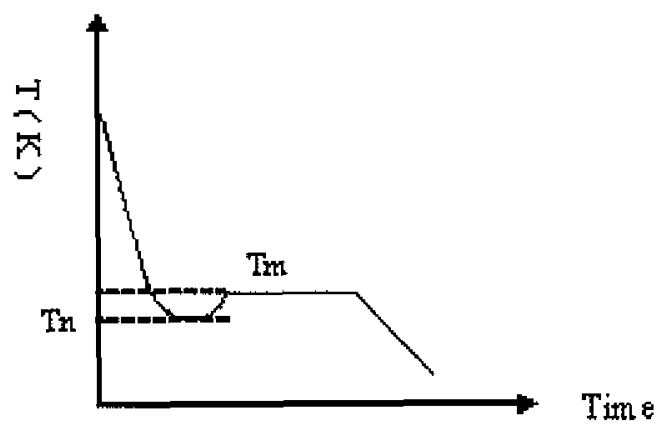

Fig. 2-1 Solidification Curve of Pure Metal at very low cooling rate

In order to start up the solidification process, the actual temperature must be lower then the melting temperature of the pure metal. This phenomenon is called "supercooling". This is a characteristic feature of $1^{\text {st }}$ order phase transformations.

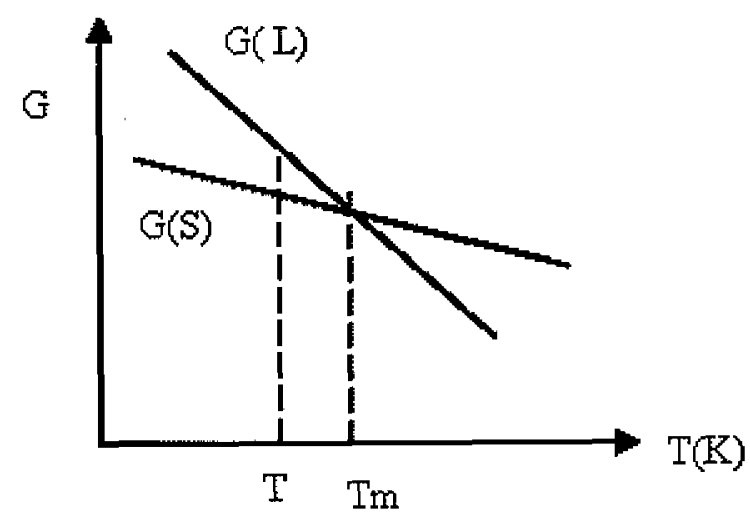

Fig. 2-2 Free Energy versus Temperature for $L \leftrightarrow S$ Transition 
From the thermodynamics of transformation, at $T m$, the free energy of liquid metal $G(L)$ is equal to that of solid metal $\mathrm{G}(\mathrm{S})$. But if the solidification can only start at some temperature less than $\operatorname{Tm}$ (supercooling), so that $\Delta \mathrm{G}=\mathrm{G}(\mathrm{S})-\mathrm{G}(\mathrm{L})<0$, the solidification process can automatically carry on at a constant temperature. At the flat plateau in the cooling curve, and this period, the liquid and the solid co-exist, here we give the second characteristic of the $1^{\text {st }}$ order transition, coexistence. The explanation for the appearance of the flat plateau is that during the phase transformation process, it releases the latent heat that is equal to the heat of the system lost to the environment. Therefore, latent heat is another characteristic for the $1^{\text {st }}$ order transition. Only when all the liquid has transformed into the solid, the temperature will decrease further, this process is completed via a dynamic atomic diffusion process between the liquid state and the solid state. If on the other hand the transition is from the solid to the liquid phase, it needs a superheating to initiate the process. Then, the final result is that there exists a temperature hysteresis for the cooling and heating process. Therefore, a temperature hysteresis is another signature of the first order transformation.

There is an important law for the first order transition, called Clausius-Clapeyron equation which is given in many thermodynamics textbooks:

$$
\frac{d T}{d(\ln P)}=\frac{R T^{2}}{\Delta H}
$$

Where $\mathrm{R}$ is the gas constant, $\mathrm{H}$ is the heat of vaporization for liquid-vapor transformation [9]. As we shall see the Curie point transition for some range of compositions of the alloys exhibits a first order transition which will be governed by this equation. It is clear from equation 2-1 that the temperature can be changed by the pressure P. Later, we will see a similar equation for a second order transition in magnetic materials. 


\subsection{Second Order Transition: Paramagnetic $\leftrightarrow$ Ferromagnetic}

As discussed before, for magnetic materials, the competition between thermal energy and exchange energy between magnetic moments determines whether it is in an order state or in a disordered state. For $\mathrm{T} \gg \mathrm{Tc}$, thermal energy destroys all orientation orders, and $\mathrm{M}$ is zero. For $\mathrm{T}$ slightly greater than $\mathrm{Tc}$, magnetic moments show a short-range order in some magnetic moment clusters. It needs a strong external magnetic field to increase magnetization magnitude. For $\mathrm{T}=\mathrm{T} c$, thermal energy and exchange energy balance, at this temperature, spontaneous magnetization appears. In other words, a long range magnetic order is established, and a weak magnetic field can increase the magnitude of magnetization many times.

In addition to the appearance of long-range order, there is a discontinuity change of symmetry of magnetic system. According to Laudau's theory of ferromagnetic transformation [10], we need two functions to describe the system, one is a scalar function $\rho(\mathrm{r})$, called the probability density for electron distribution at each point in the material. The other vector probability density function is $s(r)$, sometimes known as the order parameter, which can describe the density probability of atomic magnetic moments. When a paramagnetic (PM) to a ferromagnetic (FM) transition occurs, and if there is no structural change (as is normally the case), then $\rho(r)$ is unchanged. However, the $s(r)$ will be changed. Above $T c, s(r)=0$. Below Tc, $s(r) \neq 0$.

From the thermodynamic perspective, if PM to FM transition is completed under zero field, we can derive the following equations based on the classification of phase transformation. 
As pointed out before, for the $2^{\text {nd }}$ order transformation, the first derivative of $\mathrm{G}$ is continuous, but the second derivative of $\mathrm{G}$ is discontinuous.

Therefore, we have a discontinuous change in the specific heat $\mathrm{C}_{\mathrm{p}}$ :

$$
\Delta C_{p}=\left(C_{p 2}-C_{p 1}\right)=T \cdot \Delta\left(\frac{\partial S}{\partial T}\right)=-T\left(\frac{\partial^{2} G_{2}}{\partial T^{2}}-\frac{\partial^{2} G_{1}}{\partial T^{2}}\right)
$$

The second parameter with a sharp change is the compressibility:

$$
\Delta \chi=\left(\chi_{2}-\chi_{1}\right)=-\frac{1}{V}\left(\frac{\partial V_{2}}{\partial P}-\frac{\partial V_{1}}{\partial P}\right)=-\frac{1}{V}\left(\frac{\partial^{2} G_{2}}{\partial P^{2}}-\frac{\partial^{2} G_{1}}{\partial P^{2}}\right)
$$

The third parameter with a sharp change is the volume thermal expansion coefficient:

$$
\Delta \alpha=\frac{1}{V}\left(\frac{\partial V_{2}}{\partial T}-\frac{\partial V_{1}}{\partial T}\right)=\frac{1}{V}\left(\frac{\partial^{2} G_{2}}{\partial T \partial P}-\frac{\partial^{2} G_{1}}{\partial T \partial P}\right)
$$

For the $2^{\text {nd }}$ order transformation, the first derivative of $\mathrm{G}$ is continuous at the transition temperature, therefore, we get:

$$
\Delta S=-\left(\frac{\partial G_{2}}{\partial T}-\frac{\partial G_{1}}{\partial T}\right)=0
$$

and

$$
\Delta V=\left(\frac{\partial G_{2}}{\partial P}-\frac{\partial G_{1}}{\partial P}\right)=0
$$


Since $\Delta S$ and $\Delta V$ are the function of the variables $S, T$, and $P$, if we differentiate the equations above for $\Delta \mathrm{S}$ and $\Delta \mathrm{V}$ with respect to $\mathrm{P}$ and $\mathrm{T}$, then we have:

$$
\begin{aligned}
& \Delta\left(\frac{\partial^{2} G}{\partial T \partial P}\right) d P+\Delta\left(\frac{\partial^{2} G}{\partial T^{2}}\right) d T=0 \\
& \Delta\left(\frac{\partial^{2} G}{\partial P^{2}}\right) d P+\Delta\left(\frac{\partial^{2} G}{\partial T \partial P}\right) d T=0
\end{aligned}
$$

Substituting the equations above for $\Delta \mathrm{C}, \Delta \mathrm{x}, \Delta \alpha$, we can obtain:

$$
\frac{d T}{d P}=\frac{T V \Delta \alpha}{\Delta C_{p}}=\frac{\Delta x}{\Delta \alpha}
$$

At transition temperature $T_{\mathfrak{c}}$, the above equation can be revised as:

$$
\frac{d T_{c}}{d P}=\frac{T V \Delta \alpha}{\Delta C_{P}}=\frac{\Delta x}{\Delta \alpha}
$$

The above equation for $2^{\text {nd }}$ order phase transformations is analogous to the ClausiusClapeyron equation shown in (2-1) for $1^{\text {st }}$ order transformations. In addition, it is clear that Tc can be changed by the pressure, and this has been verified by the experiments [11].

In conclusion, for a PM $\leftrightarrow$ FM transition, there are several characteristics for this so-called second order transformation ( detailed classification of transformation given below): 1 . It is a point transformation, which means that a certain temperature (Curie temperature, or Neel temperature) is the border between the ordered state and the disordered state; 2 . As stated 
before, at Curie temperature, the symmetry of material changes; 3 . There is no latent heat required for a second order phase transformation. 4. At transition point, PM and FM can not co-exist.

\subsection{Characteristics of Phase Transitions}

Above, the two types of transformations have been discussed from the thermodynamics point of view. Here we give some typical thermodynamic parameter' graphs for them, so that these two type transformations can be easily distinguished from experimental data. In Fig. 2-3, the temperature variations of $G, S, V$ and $C_{p}$ are shown by four graphs for the first order transition.

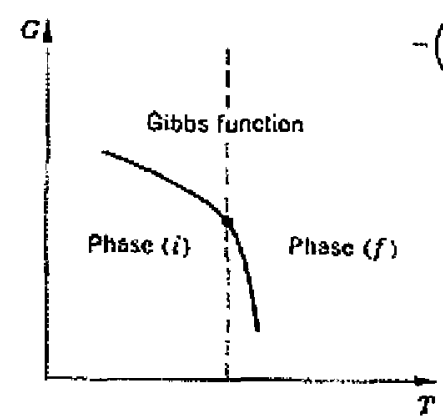

(a)

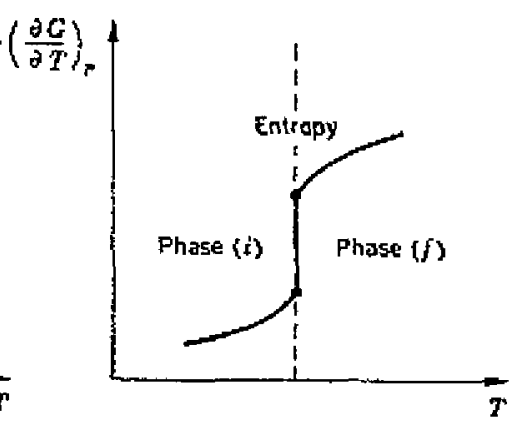

(b)

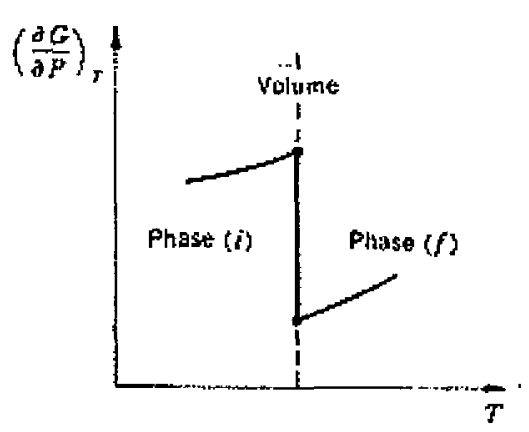

(c)

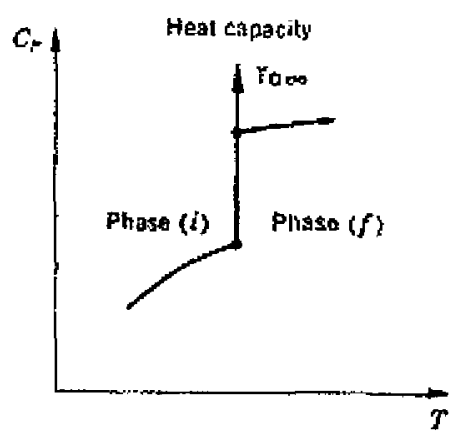

(d)

Fig. 2-3 Characteristics of a first-order phase transition. (a) Gibbs function; (b) entropy; (c) volume; (d) heat capacity, ref $[42,44]$. 
A graph of $C_{p}$ against $T$ can be used to distinguish among the types of phase transitions, as shown in Fig.2-4. The name of "lambda transition" is for the fact that the shape of $\mathrm{C}_{\mathrm{p}}-\mathrm{T}$ curve in the third graph resembles the Greek letter lambda. It can be seen in graph (a) that as a substance in any one phase approaches the temperature at which a first-order phase transition is to occur, its $\mathrm{C}_{\mathrm{p}}$ remains finite up to the transition temperature. It becomes infinite only when a small amount of the other phase is present, and its behavior before this takes place shows no evidence of any premonition of the coming event. However, in the case of a lambda transition, as is evident in graph (c), $C_{p}$ starts to rise before the transition points is reached, as though the substance, in the form of only one phase, "anticipated" the coming phase transition. A lambda transition is a special case of second order transitions. Usually, Lambda transition is also classified as the $2^{\text {nd }}$ order transition.
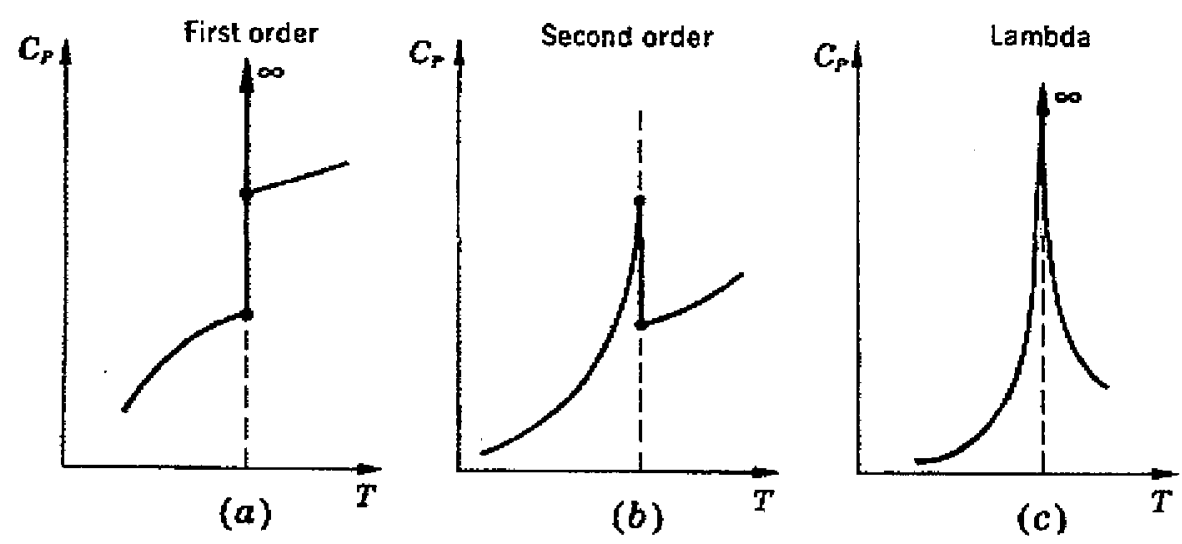

Fig. 2-4 Distinguishing characteristic among the three types of phase transitions. (a) Firstorder; (b) second-order; (c) lambda, ref [42, 44] 


\section{Chapter Three}

\section{Thermodynamics of the Magnetocaloric Effect}

In this chapter, magnetocaloric effect will be discussed. $\mathrm{Gd}_{5}\left(\mathrm{Si}_{\mathrm{x}} \mathrm{Ge}_{1-\mathrm{x}}\right)_{4}$ was found to have a giant magnetocaloric effect over part of the range of compositions. Detailed discussion of this will be given in next chapter, which shows a possible application of the material in magnetic refrigeration at near room temperature.

\subsection{Components of heat capacity}

From the purely engineering viewpoint, the heat capacity is the rate of change of internal energy with temperature, sometimes, written as "the amount of heat energy required to raise the temperature by one degree". However, this heat capacity has various components, and in case of a magnetic material, we can write:

$$
C_{t o t}=C_{e}+C_{l}+C_{m}
$$

where $C_{1}$ is the lattice specific heat, $C_{e}$ is the electronic specific heat and $C_{m}$ is the magnetic specific heat.

The lattice specific heat $C_{1}$ is expected to obey a Debye law which describes the vibrations of the ionic lattice in terms of simplified quantities such as the frequency of vibration $\omega$, the mass of the atoms $\mathrm{m}$ and the lattice stiffness (or force coefficient) $\mathrm{k}$. At higher temperature, $\mathrm{C}_{\mathrm{I}}=3 \mathrm{Nk}_{\mathrm{B}}$, which is the classical limit. 
The electronic specific heat is usually a much smaller contribution than the lattice specific heat, and in simplified case of a "free electron" solid, this electronic specific heat is given by

$$
C_{e}=\frac{\pi^{2}}{2} \frac{N \kappa_{B}{ }^{2} T}{E_{F}}
$$

where $\mathrm{N}$ is the total number of conduction electrons per unit volume, $\mathrm{T}$ is the thermodynamic temperature and $\mathrm{E}_{\mathrm{F}}$ is the energy at the Fermi level. This means that the number of electrons which can contribute to the heat capacity is much lower than the number of conduction electrons, and the contribution depends linearly on $\mathrm{T}$, meaning that as $\mathrm{T}$ is increased, more electrons come within range of being thermally excitable into available energy states and therefore $\mathrm{C}_{\mathrm{e}}$ can increase with temperature.

As a result, the variation of heat capacity $C_{\text {tot }}$ non-magnetic material behaves approximately as follows.

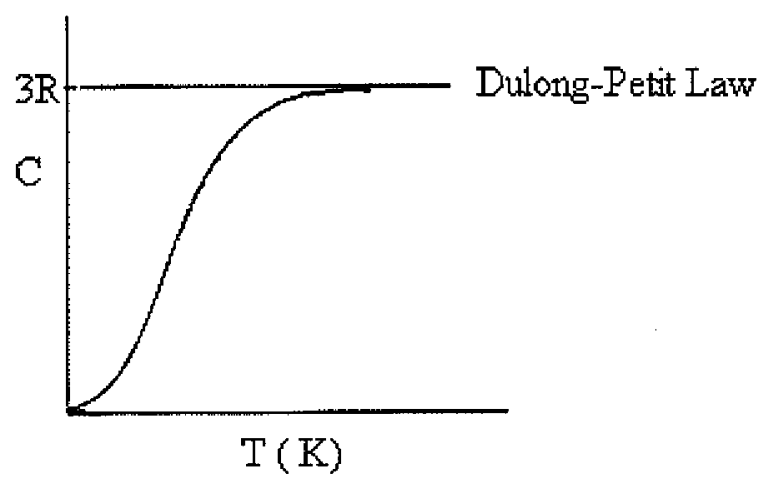

Fig. 3-1 Typical variation of heat capacity with temperature [43]

In a magnetic material, the heat capacity behaves somewhat differently with the following general shape. 


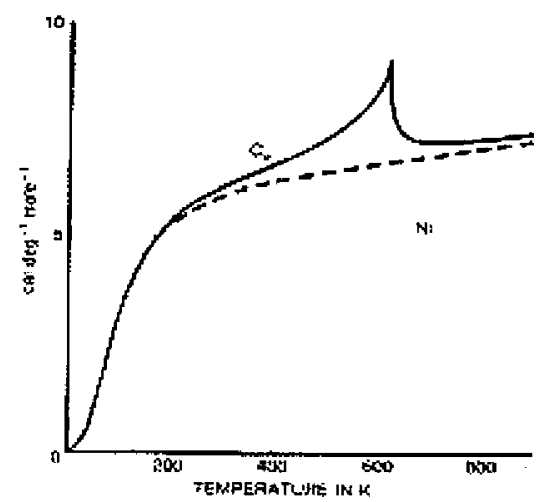

Fig.3- 2 Typical variation of heat capacity with temperature for a magnetic material [4] The magnetic contributions to the heat capacity can be determined by subtracting off the other components from the total as a non-magnetic background.

$$
C_{m}=C_{t o t}-C_{l}-C_{e}
$$

This leaves a characteristic "lambda" shape dependence of the magnetic heat capacity on temperature. See the figure below.

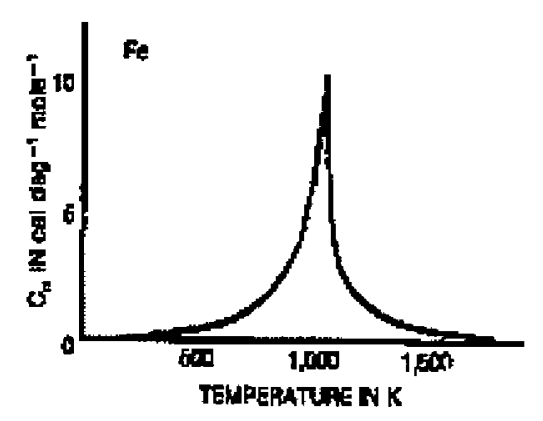

Fig. 3-3 Variation of magnetic contribution to heat capacity with temperature [4] It is this component of the heat capacity that we are particularly interested for the magnetocaloric effects and magnetic refrigeration. 


\subsection{Heat capacity, entropy change and temperature differences}

We know from the measurement of heat capacity of these materials that most of the interest will focus on the order-disorder transition temperature. The amount of heat that can be accepted or rejected by the magnetic material as it passes through the Curie temperature is

$$
\Delta Q=\int_{T_{1}}^{T_{2}} C(T) d T
$$

where of course $\mathrm{C}$ is now a strong function of temperature.

On the other hand, if the process proceeds at a fixed temperature, there can still be an amount of heat accepted or rejected simply because the heat energy can be used to change the entropy of the system. In this case, the amount of heat accepted or rejected is

$$
\Delta Q=\int_{S_{1}}^{S_{2}} T d S
$$

The exact relations between these quantities have to be handled carefully because the value of the quantity depends on which property is being held constant during the thermodynamic process.

Clearly the materials that will perform best in magnetic refrigeration are those for which the magnetic contribution the heat capacity $\mathrm{C}_{\mathrm{mag}}$ is large, or for which the change in magnetic entropy $\Delta \mathrm{S}_{\mathrm{mag}}$ is large. Measurements of either of these quantities will be indicative of a suitable material, always recognizing that the operating temperature range will be close to the Curie temperature. 
Under isothermal conditions the measurement of entropy change is useful, while under adiabatic condition, the measurement of temperature change is useful.

\subsection{Effect of magnetic field}

Application of a magnetic field can cause a change in the magnetic order in materials, such as $\mathrm{Gd}_{5}\left(\mathrm{Si}_{x} \mathrm{Ge}_{1-\mathrm{x}}\right)_{4}$. In particular, at temperatures close to the Curie temperature, a field of $\sim 1$ Tesla can be used to induce a phase transformation from the paramagnetic to the ferromagnetic phase. This will result in an effective change in temperature if conducted under adiabatic conditions, or a change in entropy if conducted under isothermal condition.

The isothermal magnetic entropy change that occurs where the material is magnetized is given by

$$
\Delta S_{\operatorname{mag}}=\int_{0}^{H}\left(\frac{\partial M}{\partial T}\right)_{H} d H
$$

which arises from the energy equation $\mathrm{E}=\mathrm{T} d \mathrm{~S}_{\mathrm{mag}}=\mathrm{M} \mathrm{dH}$, assuming that these are the only energy terms affected.

On the other hand, the total entropy change is

$$
\Delta S_{\text {tot }}=\int_{0}^{T}\left(\frac{C}{T}\right) d T
$$

which derived from the equation $\mathrm{E}=\mathrm{T} \mathrm{dS}=\mathrm{C} \mathrm{dT}$, assuming that these are the only energy terms affected.

For the magnetic entropy contribution, 


$$
\Delta S_{\text {mag }}=\int_{0}^{T}\left(\frac{C_{m a g}}{T}\right) d T
$$

So that $\mathrm{T} d \mathrm{~S}_{\text {mag }}=\mathrm{C}_{\mathrm{mag}} \mathrm{dT}=\mathrm{E}$

The expected temperature rise is therefore linked to the change in entropy through the amount of energy that is transformed.

$$
\mathrm{T} \mathrm{dS} \text { mag }=\mathrm{MdH}=\mathrm{C}_{\mathrm{mag}} \mathrm{dT}
$$

And therefore, the change in temperature in an adiabatic process is related to the change in entropy in an isothermal process by

$$
\frac{d T}{T}=\frac{d S_{m a g}}{C_{m a g}}
$$

where units of $\mathrm{C}_{\text {mag }}$ are Joules per $\mathrm{kg} \bullet$ per degree $\mathrm{K}$, and similarly the units of entropy are the same.

The magnetocaloric effect is therefore manifested either as an isothermal magnetic entropy change or an adiabatic temperature change of a magnetic material that occurs on the application of a magnetic field.

For reversible process, the Maxwell relation can be used,

$$
\left(\frac{\partial S}{\partial B}\right)_{T}=\left(\frac{\partial M}{\partial T}\right)_{B}
$$

The amount of heat transferred between two heat sinks (hot and cold) at temperatures, $T_{2}$ and $T_{1}$ is

$$
\Delta Q=\int_{T_{1}}^{T_{2}} \Delta S_{\operatorname{mag}}(T) d T
$$


The change in the magnetic entropy $\Delta \mathrm{S}_{\mathrm{m}}$ is given by

$$
\Delta S_{m}=\int_{0}^{T} \frac{C(T)}{T} d T
$$

or equivalently if there is a change in magnetic field, this is given by

$$
\Delta T=-\int_{0}^{B} \frac{T}{C(T)}\left(\frac{\partial M}{\partial T}\right) d B
$$

\subsection{Magnetocaloric effect}

When a magnetic material is subjected to an applied field, the orientation of the magnetic moments (spins) is changed, and if the material is close to its Curie temperature, these changes can be quite large, resulting in ordering of the magnetic structure from an initially disordered state.

There is an entropy associated with the magnetic degree of freedom, and this is denoted by the "magnetic entropy" $\mathrm{S}_{\mathrm{m}}$. Under adiabatic change, for example where a magnetic field is applied suddenly and there is no opportunity to reach a thermodynamic equilibrium, the change in magnetic entropy must be exactly compensated by a change in lattice entropy. This means that there will be an increase in temperature, $\Delta \mathrm{T}$, which is known as the magnetocaloric effect. As a result, heat can be rejected from this material. Similarly if the magnetic field is removed suddenly, the temperature decreases and so heat can be absorbed. It is important to note that as a result of these processes heat can be transferred from a cold sink to a hot sink, the principle of refrigeration. In later chapter, the magnetic refrigeration will be further discussed when we discuss $\operatorname{Gd}_{5}\left(\mathrm{Si}_{\mathrm{x}} \mathrm{Ge}_{1-\mathrm{x}}\right)_{4}$, a material has a giant magnetocaloric effect. 


\section{Chapter Four}

\section{Introduction to $\mathrm{Gd}_{5}\left(\mathrm{Si}_{\mathrm{x}} \mathbf{G e}_{1-\mathrm{x}}\right)_{4}$ system}

In the previous chapter, we have discussed the magnetocaloric effect: When a magnetic material is subjected to an external magnetic field, the material will release the heat into the environment. When the applied magnetic field is removed, the magnetic material will absorb heat from the environment. In recent years, the magnetocaloric effect, especially giant magnetocaloric effect (GMC) has triggered a lot of research interests since the discovery of GMC effect in $\operatorname{Gd}_{5}\left(\mathrm{Si}_{\mathrm{x}} \mathrm{Ge}_{1-\mathrm{x}}\right)_{4}$ system $(0 \leq \mathrm{x} \leq 0.503)$ by Pecharsky, Gschneidner, et al in 1997 [12], later on, the GMC effect was also reported on $\mathrm{MnFeP}_{0.45} \mathrm{As}_{0.55}$ by Tegus, et al in 2002 [13]. It was found that for these two types' materials, both of their GMC effects are the results of the so-called first order magneto-structural transformations [13].

Pecharsky, et al proposed a phase diagram for the as-prepared pseudo-binary for $\mathrm{Gd}_{5}\left(\mathrm{Si}_{\mathrm{x}} \mathrm{Ge}_{1}\right.$ x) $)_{4}$ in 2002 [14], as shown in Fig. 4-1, which is the refined crystal phase diagram proposed in ref [12]. As seen in this diagram, $\mathrm{Gd}_{5}\left(\mathrm{Si}_{x} \mathrm{Ge}_{1-\mathrm{x}}\right)_{4}$ has a complex magnetic and crystal structure depending on the $\mathrm{x}$ value and temperature. As for magnetic structure, it could be in paramagnetic, ferromagnetic and antiferromagnetic state. As for crystalline structure, it could be two types, orthorhombic structure and monoclinic structure. Pecharsky, et al [14], [15] have divided the phase diagram into three non-shaded main regions from the left to the right as shown in Fig.4-1:

(I) $0<x \leq 0.3$

In this Ge rich region, at higher temperature, it is in paramagnetic state with a so-called $\mathrm{Gd}_{5}\left(\mathrm{Ge}_{4}\right)$ type orthorhombic crystal structure, which was discussed in details in Choe, et al 
[16], as shown in Fig. 4-2. At intermediate temperature range, it is in antiferromagnetic state with $\mathrm{Gd}_{5}\left(\mathrm{Ge}_{4}\right)$ type structures. At lower temperature range, it is in ferromagnetic state with a different crystal structure: $\mathrm{Gd}_{5}\left(\mathrm{Si}_{4}\right)$ type orthorhombic structure with space group Pnma. According to Choe in ref [16], the only difference between the $\mathrm{Gd}_{5}\left(\mathrm{Ge}_{4}\right)$ type and the $\mathrm{Gd}_{5}\left(\mathrm{Si}_{4}\right)$ type is that the $\mathrm{Si}(\mathrm{Ge})-\mathrm{Si}(\mathrm{Ge})$ bonds between the adjacent slabs in $\mathrm{Gd} 5(\mathrm{Ge} 4)$ type are all broken, while in Gd5(Si4) type, they are all connected together, as shown in Fig. 4-2.

\section{(II) $0.4<x \leq 0.503$}

In this region, at high temperature, it is a monoclinic structure and a paramagnet with space group $\mathrm{P} 112_{1} / \mathrm{a}$, also called $\mathrm{Gd}_{5}\left(\mathrm{Si}_{2} \mathrm{Ge}_{2}\right)$ type. As shown in the Fig. 4-2, some $\mathrm{Si}(\mathrm{Ge})-\mathrm{Si}(\mathrm{Ge})$ bonds between the layers are broken. When it was cooled down, the crystal structure changed into the $\mathrm{Gd}_{5}\left(\mathrm{Si}_{4}\right)$ type (space group Pnma) and a ferromagnet, and the broken bonds are connected together.

\section{(III) $0.575 \leq x \leq 1$}

In this $\mathrm{Si}$ rich region, when it is cooled down from high temperature to low temperature, it remains its magnetic and crystal structure: $\mathrm{Gd} 5$ (Si4) type orthorhombic structures ( space group Pnma) with all $\mathrm{Si}(\mathrm{Ge})-\mathrm{Si}(\mathrm{Ge})$ bonds connected together between the layer, as shown in Fig. 4-2.

The graded regions shown in Fig. 4-1 which is the two-phase region, and was found that the heat treatment on the as-prepared alloys compound has considerable effect on the phase diagram [14]. Much research work, including the work done in this thesis, has demonstrated that the phase transition diagram depends on many factor, such as the purity of starting materials, the external magnetic field, hydrostatic pressure, materials preparing methods, heat treatment, and so on, and these effects will be discussed in the later chapters. 


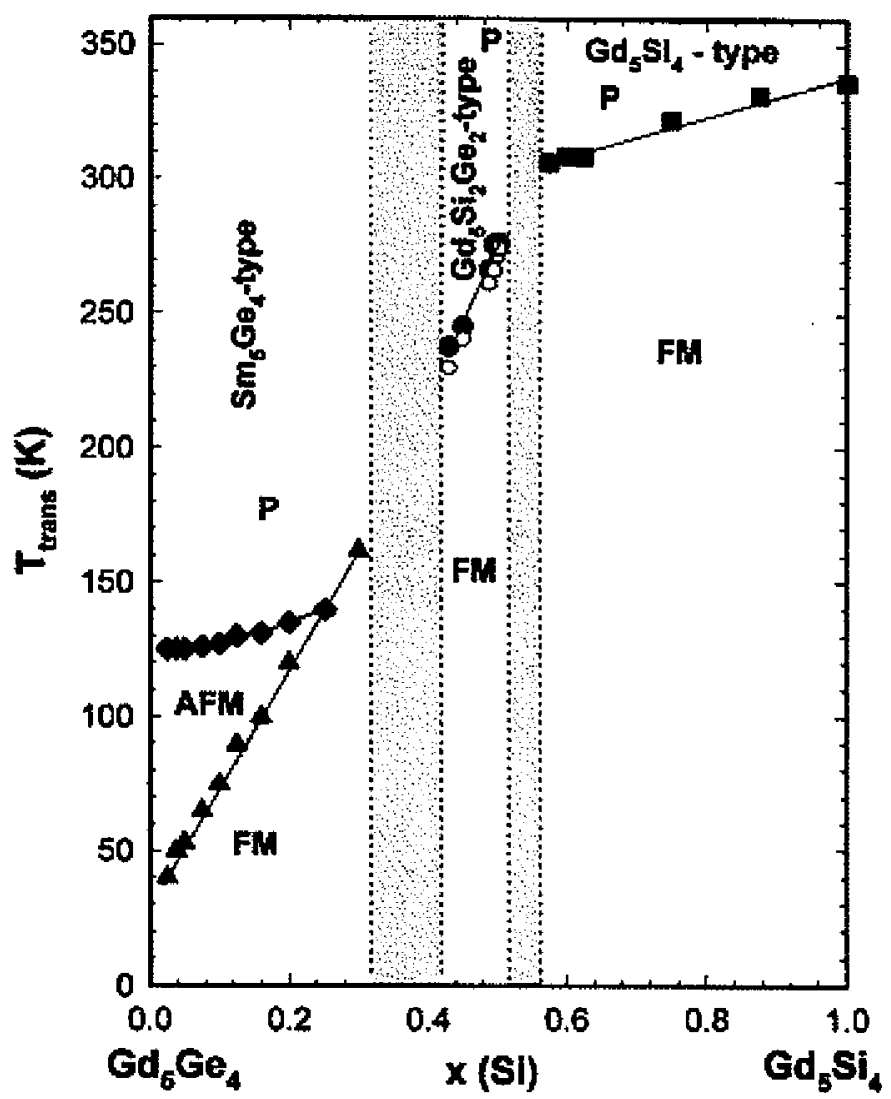

Fig. 4-1 Magnetic and crystalline structure phase diagram for $G d_{5}\left(\mathrm{Si}_{x} G e_{1-x}\right)_{4}$ proposed by A.O. Pecharsky, et al. in ref [14]. 


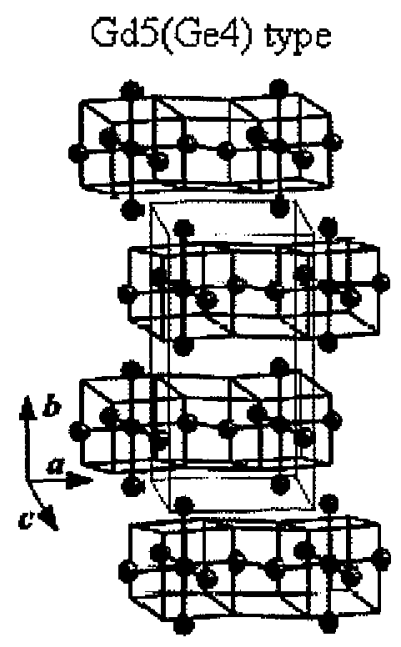

Paramagnet

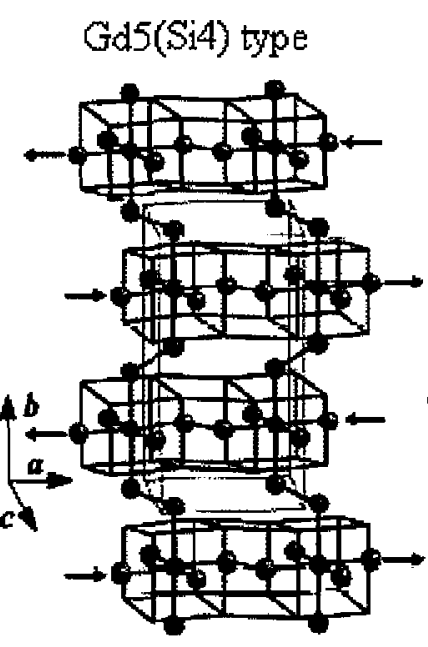

Ferromagnet

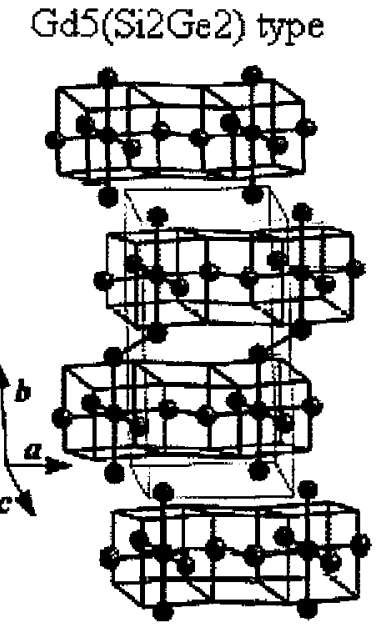

Paramagnet

Fig. 4-2 Crystal structure of $\mathrm{Gd}_{5}\left(\mathrm{Si}_{\mathrm{x}} \mathrm{Ge}_{1-\mathrm{x}}\right)_{4}$. The green atoms are $\mathrm{Gd}$, The red and blue atoms are $\mathrm{Si}$ or $\mathrm{Ge}$ [14] [17].

Pecharsky, et.al have also published the crystalline data for $\operatorname{Gd}_{5}\left(\mathrm{Si}_{\mathrm{x}} \mathrm{Ge}_{1-\mathrm{x}}\right)$ 4, which is shown in the following Table. The data will be helpful for us to explain the thermal expansion results when the transition occurs, therefore, it is given here. 
Table 4: The crystallographic data for $\mathrm{Gd}_{5}\left(\mathrm{Si}_{x} \mathrm{Ge}_{1-x}\right)_{4}$, published in ref [14]

\begin{tabular}{|c|c|c|c|c|c|c|}
\hline \multirow[t]{2}{*}{ Composition } & \multirow{2}{*}{$\begin{array}{l}\text { Space } \\
\text { group }\end{array}$} & \multicolumn{3}{|c|}{ Lattice paranneters $(\hat{A})$} & \multirow[t]{2}{*}{$\gamma\left({ }^{\circ}\right)$} & \multirow{2}{*}{$\begin{array}{l}\text { Unit cell } \\
\text { volume }\left(\alpha^{3}\right)\end{array}$} \\
\hline & & $a$ & $b$ & $c$ & & \\
\hline \multicolumn{7}{|c|}{$\mathrm{Sm}_{5} \mathrm{Ge}_{4}-\mathrm{type}$ structure } \\
\hline $\mathrm{Gd}_{5} \mathrm{Ge}_{2}{ }^{\mathrm{a}}$ & Pnma & $7.6968(5)$ & $14.831(1)$ & $7.7851(1)$ & -- & $888.7(2)$ \\
\hline Gds(Sio.0s $\left.\mathrm{Ge}_{0.995}\right)_{4}$ & Pnna & $7.6967(6)$ & $14.831(1)$ & $7.7819(6)$ & - & $888.3(2)$ \\
\hline $\mathrm{Gd}_{5}\left(\mathrm{Si}_{0.025} \mathrm{Ge}_{0.9 n s}\right)_{4}$ & Pnma & $7.6953(5)$ & $14.830(1)$ & $7.7829(6)$ & - & $888.2(2)$ \\
\hline $\mathrm{Gd}\left(\mathrm{Si}_{0,038} \mathrm{Ge}_{0.962}\right)_{4}$ & Pnma & $7.6951(5)$ & $14.826(1)$ & $7.7816(5)$ & - & $887.8(2)$ \\
\hline $\mathrm{Gd}_{5}\left(\mathrm{Si}_{0.050} \mathrm{Ge}_{0.950}\right)_{4}$ & Pnmo & $7.6939(5)$ & $14.827(1)$ & $7.7812(6)$ & - & $887.7(2)$ \\
\hline Gds(Sions Geo.92s) & Pnna & $7.6893(5)$ & $14.822(1)$ & $7.7782(5)$ & - & $886.5(2)$ \\
\hline $\mathrm{Gds}\left(\operatorname{Si}_{0.083} \operatorname{Ge}_{0.917}\right)_{4} \mathrm{a}$ & Pnma & $7.689(1)$ & $14.822(1)$ & $7.777(2)$ & - & $886.4(3)$ \\
\hline $\mathrm{Gds}_{5}\left(\mathrm{Si}_{0.100} \mathrm{Ge}_{0.900}\right)_{4}$ & Pnma & $7.6881(7)$ & $14.822(1)$ & $7.7763(7)$ & - & $886.2(2)$ \\
\hline $\mathrm{Gds}_{5}\left(\mathrm{Si}_{0.125} \mathrm{Gre}_{0.875}\right)_{4}$ & Pnma & $7.6858(4)$ & $14.817(1)$ & $7.7752(5)$ & - & $885.5(2)$ \\
\hline Gds $\left(S_{10.150} G_{0.050}\right)_{s}$ & Pnma & $7.6868(5)$ & $14.819(1)$ & $7.7738(6)$ & - & $885.4(2)$ \\
\hline $\mathrm{Gds}_{5}\left(\mathrm{Si}_{0.160} \mathrm{Ge}_{0.840}\right)_{4}$ & $P_{n m a}$ & $7.6797(4)$ & $14.816(1)$ & $7.7713(5)$ & - & $884.2(2)$ \\
\hline Gds(Sio.225 Geo.77s) & Pnma & $7.6745(4)$ & $14.811(1)$ & $7.7670(4)$ & - & $882.8(2)$ \\
\hline $\operatorname{Gds}\left(\operatorname{Si}_{0.253} \operatorname{Gec}_{0.747}\right)_{4}$ & Pnma & $7.6689(6)$ & $14.804(1)$ & $7.7609(6)$ & - & $881.1(2)$ \\
\hline $\mathrm{Gd}_{5}\left(\mathrm{Si}_{0.300} \mathrm{Ge}_{0,700}\right)_{4}$ & Pnma & $7.6617(6)$ & $14.801(1)$ & $7.7604(6)$ & - & $880.1(2)$ \\
\hline \multicolumn{7}{|c|}{$\mathrm{Gd}_{5}\left(\mathrm{Si}_{2} \mathrm{Ge}_{2}\right)$-type structure } \\
\hline $\mathrm{Gd}_{5}\left(\mathrm{Si}_{410} \mathrm{Ge}_{5} \mathrm{~S}_{2}\right)_{4} \mathrm{a}$ & $P 112_{\jmath} / a$ & $7.5906(7)$ & $14.810(1)$ & $7.7864(8)$ & $93.146(5)$ & $874.0(2)$ \\
\hline $1570 \mathrm{~K} / 1 \mathrm{~h}$ & $P 112_{3} / a$ & $7.5932(6)$ & $14.812(1)$ & $7.7843(7)$ & $93.202(4)$ & $874.1(2)$ \\
\hline $\mathrm{Gds}\left(\mathrm{Si}_{0.450} \mathrm{Ge}_{0.550}\right)_{4}$ & $P 112 / a$ & $7.5904(7)$ & $14.810(1)$ & $7.7830(4)$ & $93.191(4)$ & $873,6(3)$ \\
\hline $1570 \mathrm{~K} / 1 \mathrm{~b}$ & $P 112, / a$ & $7.5910(6)$ & $14.811(1)$ & $7.7828(4)$ & $93.197(4)$ & $873.7(2)$ \\
\hline $\mathrm{Gd}_{5}\left(\mathrm{Si}_{0.488} \mathrm{Ge}_{0.512}\right)_{4}$ & $P 112, / a$ & $7.5859(6)$ & $14.808(1)$ & $7.7817(6)$ & $93.173(2)$ & $872.8(2)$ \\
\hline $1570 \mathrm{~K} / 1 \mathrm{~h}$ & $P 112, / a$ & $7.5873(5)$ & $14.808(1)$ & $7.7819(5)$ & $93.216(3)$ & $872.9(2)$ \\
\hline $\mathrm{Gd}_{5}\left(\mathrm{Si}_{0,4 \% 6} \mathrm{Geos}_{05}\right)_{4}$ & $P 112, / a$ & $7,5835(6)$ & $14.807(1)$ & $7.7815(6)$ & $93.182(4)$ & $872.4(2)$ \\
\hline $1570 \mathrm{~K} / 1 \mathrm{~h}$ & $P 112 / a$ & $7.5845(6)$ & $14.807(1)$ & $7.7802(7)$ & $93.305(4)$ & $872.4(2)$ \\
\hline $\mathrm{Gd}_{5}\left(\mathrm{Si}_{0.500} \mathrm{Ge}_{0.500}\right)_{4}$ & $P 112, / a$ & $7.5854(7)$ & $14.802(2)$ & $7.7800(8)$ & $93.176(4)$ & $872.5(3)$ \\
\hline $1570 \mathrm{~K} / 1 \mathrm{~h}$ & $P 112, / a$ & $7.5863(6)$ & $14.810(1)$ & $7.781(6)$ & $93.200(4)$ & $872.8(2)$ \\
\hline $\mathrm{Gd}_{5}\left(\mathrm{SL}_{\mathrm{SO}} \mathrm{Ge}_{0.497}\right)_{4}$ & $P 112_{1} / a$ & $7.5825(5)$ & $14.806(1)$ & $7.7798(6)$ & $93.182(4)$ & $872.1(2)$ \\
\hline $1570 \mathrm{~K} / 1 \mathrm{~h}$ & $P 112_{1} / a$ & $7.5838(6)$ & $14.806(1)$ & $7.7797(7)$ & $93.199(4)$ & $872.1(2)$ \\
\hline \multicolumn{7}{|c|}{$\mathrm{Gd}_{5} \mathrm{Si}_{4}-\mathrm{type}$ structure } \\
\hline$G d_{5}\left(S_{0.575} G_{0.425}\right)_{4}$ & Pnma & $7.5119(5)$ & $14.788(1)$ & $7.7963(4)$ & - & $866.0(?)$ \\
\hline $\mathrm{Gd}_{5}\left(\mathrm{Si}_{0.600} \mathrm{Ge}_{0.400}\right)_{4}$ & Prima & $7.5084(5)$ & $14.782(1)$ & $7.7956(5)$ & - & $865.2(2)$ \\
\hline $\mathrm{Gd}_{5}\left(\mathrm{Si}_{0.625} \mathrm{Ge}_{3755}\right)_{2}$ & Pnma & $7.5059(5)$ & $14.775(1)$ & $7.7839(5)$ & $\ldots$ & $863.2(2)$ \\
\hline $\mathrm{Gd}_{5}\left(\mathrm{Si}_{0.750} \mathrm{Ge}_{0.750}\right)_{4}$ & Pnma & $7.4972(5)$ & $14.765(1)$ & $7.7709(5)$ & - & $860.2(2)$ \\
\hline $\mathrm{Gd}_{5}\left(\mathrm{Si}_{0.800} \mathrm{Ge}_{0.200}\right)_{4}^{*}$ & Pruma & $7.4963(9)$ & $14.765(2)$ & $7.771(1)$ & - & $860.1(3)$ \\
\hline $\mathrm{Gd}_{5}\left(\mathrm{Si}_{0875} \mathrm{Ge}_{0.125}\right)_{4}$ & Pnma & $7.4902(5)$ & $14.7522(9)$ & $7.7559(5)$ & - & $857.0(2)$ \\
\hline $\mathrm{Gd}_{5} \mathrm{Si}_{4}{ }^{2}$ & Pnma & $7.4822(4)$ & $14.7396(7)$ & $7.7453(4)$ & - & $854.2(1)$ \\
\hline
\end{tabular}

The above crystallographic data published by Pecharsky et al. for single phase $\mathrm{Gd}_{5}\left(\mathrm{Si}_{\mathrm{x}} \mathrm{Ge}_{1-\mathrm{x}}\right)_{4}$ in ref [14]. 


\section{Chapter Five}

\section{Thermal Expansion and Magnetostriction}

\subsection{Thermal Expansion}

The thermal expansion coefficient is a basic physical property which can be of considerable importance in mechanical and structural design applications of a material. In most solid state physics textbooks, a classical model assumes that it is the anharmonic terms in the interatomic potential energy that affect the thermal expansion coefficient. In this model, the potential energy of an atom, $\mu(x)$, is approximated by the following equation $[18,19]$.

$$
\mu(x)=c x^{2}-g x^{3}-f x^{4}
$$

where $\mathrm{c}, \mathrm{g}$, and $\mathrm{f}$ are all positive constants, $\mathrm{x} \equiv \mathrm{R}-\mathrm{R}_{0}, \mathrm{R}_{0}$ is the equilibrium separation at $\mathrm{T}=$ $0 \mathrm{~K}$, where $\mu(\mathrm{x})$ is minimized. $\mathrm{R}$ is the interatomic distance at temperature $\mathrm{T}$. Based on this model, and using the Boltzmann distribution, the mean value of $\mathrm{x}$ can be expressed as following equation.

$$
<x>=\frac{\int_{-\infty}^{+\infty} x e^{-\mu(x) / k T} d x}{\int_{-\infty}^{+\infty} e^{-\mu(x) / k T} d x}
$$

where $\mathrm{k}$ is the Boltzmann constant. The coefficient of thermal expansion $\alpha$ is given as:

$$
\alpha=\frac{1}{R_{0}} \frac{d<x>}{d T}
$$

and linear thermal expansion $<\mathrm{x}>$ is given as:

$$
<x>=\frac{3 g}{4 c^{2}} k T
$$


From this equation, $<x>$ should be linearly dependent on the temperature $\mathrm{T}$. This model works very well at high temperature, but fails in low temperature. Due to this shortcoming, in ref [20], a new model was proposed by using the Lennard-Jones potential model for a diluted-gas solid, where the measured data fits the calculated data very well for a whole temperature range.

Thermal expansion measurements are also useful for investigating phase transitions in alloys. Nix and Shockley [21] first suggest that the state of order could affect the lattice vibrations through a change in Debye temperature, and an equation has been given to show the vibrational entropy difference between an ordered and disordered phase of an alloy:

$$
\Delta S_{v i b}=S_{v i b}^{D}-S_{v i b}^{o}=3 R \ln \left(\frac{\Theta^{0}}{\Theta^{D}}\right)
$$

Where $\Theta$ is the Debye temperature and the superscripts $O$ and $D$ refer to the ordered and disordered phases, respectively. Considerable effort has been put into understanding the significant contribution of vibrational entropy to the phase stability in alloys. For instance, it has been shown that a sizable reduction in the critical temperature for ordering can been achieved if the vibrational entropy terms is incorporated in the free energy [22, 23, 24]. Furthermore, according to the Bethe-Slater curve, the magnetic state is dependent on the interatomic spacing, as shown in the following figure. Pauli's exclusion principle demands the two electrons which are too close must have opposite spins, i. e., antiferromagnetism is favored when the inter-atomic spacing is small. Parallel spin is favored if electrons share the same wavefunction but confined to separate regions of space, shown in the Fig. 5-1. 


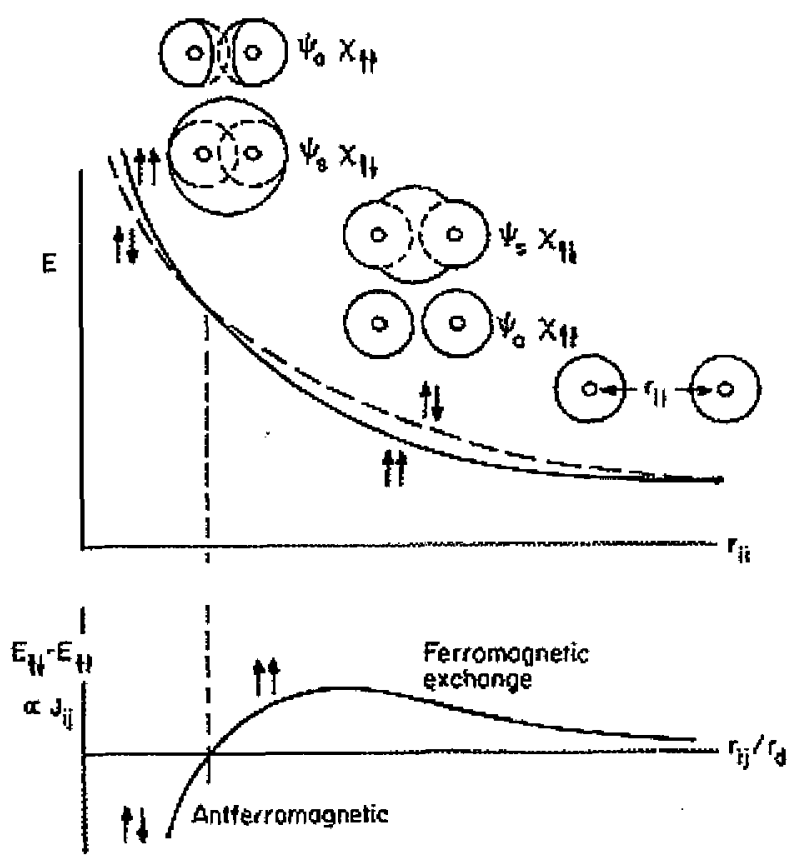

Fig.5-1 Dependence of energy of spatially symmetric and antisymmetric wavefunctions versus atomic spacing $\mathrm{r}_{\mathrm{ij}}$ (After O'Handley [25])

In conclusion, thermal expansion is a useful measurement for us to investigate the phase transition in magnetic solids and non-magnetic solids.

Fig.5-2 (a) shows the thermal expansion of a nonferromagnetic material Au. The dots were obtained from experiment, the solid line was the fitting curve based on Grueneisen theory, detailed description can be found in ref [45]. From equation (5-4), we expect that the thermal expansion is linearly dependent at some low temperature and high temperature range. Fig.5-2 (b) shows the thermal expansion of a ferromagnetic material Ni. Same as the Fig. 5-2 (a), the dots are experimental results points, the solid lines is the fitting curve of Grueneisen theory. As seen from Fig.5-2 (b), the experimental results show a larger difference from the fitting curve at the temperature range of $325 \mathrm{~K}$ and $800 \mathrm{~K}$, especially a peak appearing at $\mathrm{T}=635 \mathrm{~K}$, 
which was identified as the Curie temperature. Above $\mathrm{Tc}$, because the value of local magnetic moment is not equal to zero, but will reach to zero gradually, i. e., it gradually loses its long-range ordering, that is why we see a tail at temperature higher than Tc.

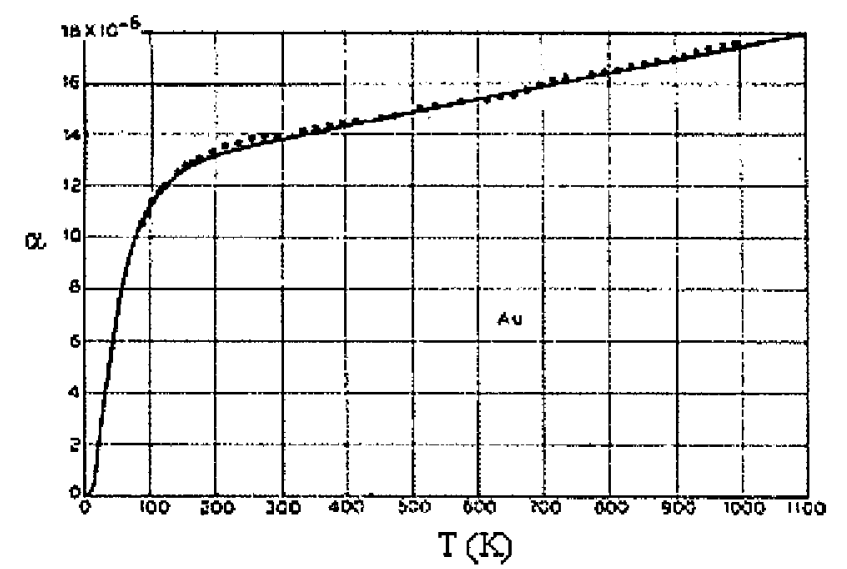

Fig.5-2 (a) Thermal expansion of $\mathrm{Au}$, where $\gamma$ is the thermal expansion coefficient. The picture is from ref [45]

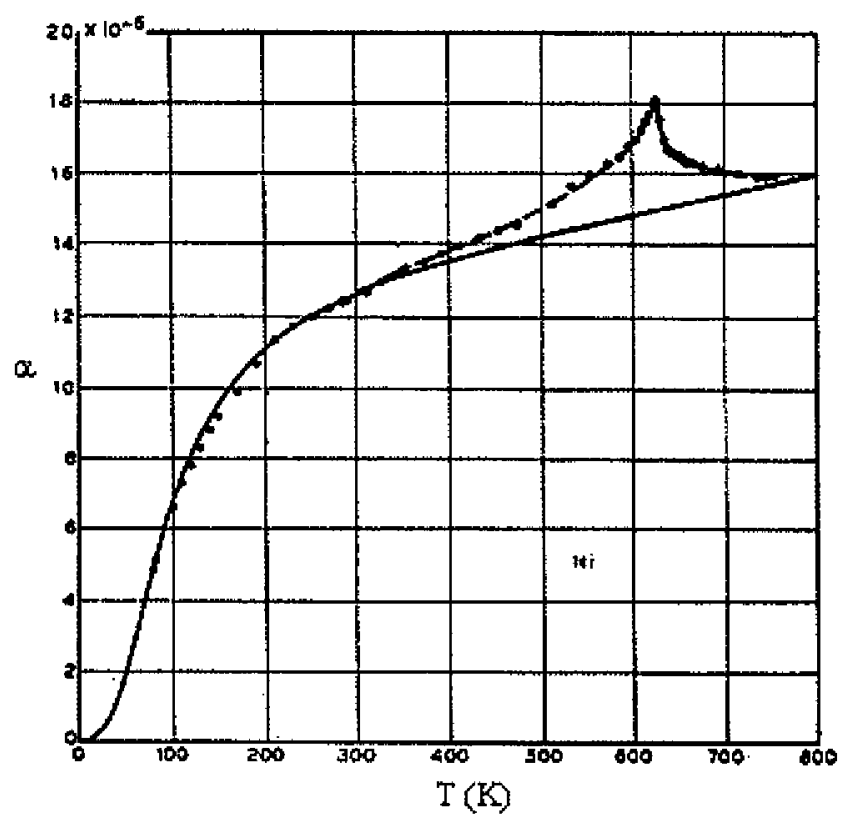

Fig.5-2 (b) Thermal expansion of ferromagnetic material Ni, [45, 26]. The picture is from [45].

Below Tc, additional magnetovolume effects due to long-range magnetic ordering occur; they may add to the volume expansion due to the presence of a local moment. If we derivate 
curve in the Fig. 5-2, a discontinuity in slope exists at $T_{\mathfrak{c}}$. The volume expansion, which is expressed as $\omega=\Delta \mathrm{V} / \mathrm{V}$, if the material is isotropic, then the volume thermal expansion will be 3 times that of linear thermal expansion. The linear coefficient of thermal expansion, $\alpha=$ $\Delta \mathrm{l} /(\mathrm{l} \times \Delta \mathrm{T})$, has also been measured for Ni by Killie [26]. For some materials with $\mathrm{Tc}$ above room temperature, the magnetovolume effects associated with long-range order turn on a positive strain below Tc. For some alloy, its thermal expansion coefficient can be zero, such an alloy is called invar alloys, for example $\mathrm{Fe}_{70} \mathrm{Ni}_{30}$.

\section{2 Magnetostriction}

\section{(1) Introduction}

When a magnetic material is exposed to a magnetic field, its dimensions change, this phenomena was first found in nickel sample by James Joule in 1842 [27]. This effect is called "magnetostriction". Magnetostriction can be classified into different deformation modes for different crystal symmetry. The following is the 4 normal deformation modes for cubic symmetry.

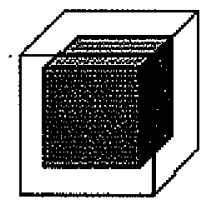

(1)

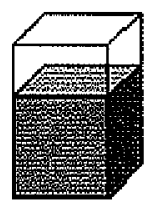

(2)

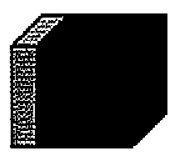

(3)

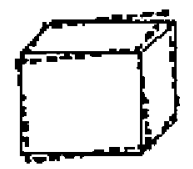

(4)

Fig. 5-3 Four modes of magnetostriction, (1) volume magnetostriction (2) elongation (3) contraction, (4) shear

Through the magnetostriction mechanisms, magnetic energy can be converted into mechanical energy and vice versa. The physics behind magnetostriction is magnetoelastic 
coupling which will be discussed later. There are some other effects which are related to the magnetoelastic coupling. By applying a stress on a magnetic material, the magnetization of material will also be altered, this effect is called the Villari effect. By passing a current longitudinally through the material to cause a circulating magnetic field, and superimposing on this an axial magnetic filed, the magnetic material will produce a torque, known as the Wiedemann effect. Conversely by applying a torque on magnetic material, a helical magnetic field results in the magnetic material. This effect is called the Matteucci effect. These magnetoelastic effects can be found useful in many magnetic sensor applications.

\section{(2)Spin-Orbit Coupling}

Phenomenologically, magnetostriction can be divided into two types: spontaneous magnetostriction which arises from the ordering of magnetic moments into domains at the Curie temperature, and field-induced magnetostriction arising from the reorientation of domains. Field-induced magnetostriction is the more interesting feature of magnetostrictive materials for applications. Spontaneous magnetostriction within domains arises from the creation of domains as the temperature of the ferromagnet passes through the Curie temperature. Field-induced magnetostriction arises when domains that have spontaneous magnetostriction are reoriented under the action of a magnetic field. On the fundamental level, magnetostriction is mainly due to spin-orbit coupling: when an external field tries to reorient the spin of an electron, the orbit of that electron also tends to be reoriented. The orbit is strongly coupled to the lattice and therefore causes a change in dimensions as it is reoriented. Because the orbit is strongly coupled to the lattice, it also resists the attempt to rotate the spin axis. This coupling is thus also responsible for magnetocrystalline anisotropy. 
It is relatively weak, because applied fields of a few hundred Oersteds usually suffice to rotate the spins away from the magnetic easy direction. The relation between magnetostriction and spin-orbit coupling can be simply shown in the Fig. 5-4, which is a row of atoms in a crystal. The black dots represent atomic nuclei. The arrows show the net magnetic moment per atom, and the oval lines enclose the electrons belonging to, and distributed nonspherically about each nucleus. The upper row of atoms depicts the paramagnetic state above $T_{c}$. If we assume that the spin-orbit coupling is very strong, then the effect of the spontaneous magnetization occurring below $T_{c}$ would be to rotate the spins and the electron clouds into some particular orientation determined by the crystal anisotropy, left to right, say. The nuclei would be forced further apart, and the spontaneous magnetostriction would be $\Delta \mathrm{L} / \mathrm{L}$. If we then apply a strong field vertically, the spins and electron clouds would rotate through 90 degrees, and the domains of which these atoms are a part would magnetostrictively strain by an amount $\Delta \mathrm{L} / \mathrm{L}$. The rare earth metals are exceptions to the above statements. Many of them are only ferromagnetic at temperatures far below room temperature, and their orbital moments are not quenched, i.e., the spin-orbit coupling is strong. Moreover, the electron cloud about each nucleus is decidedly non-spherical. When an applied field rotates the spins, the orbits rotate too and considerable distortion results. Through this interaction, crystal energy can be reduced in the so-called magnetoelastic energy via the magnetostrictive deformation. 


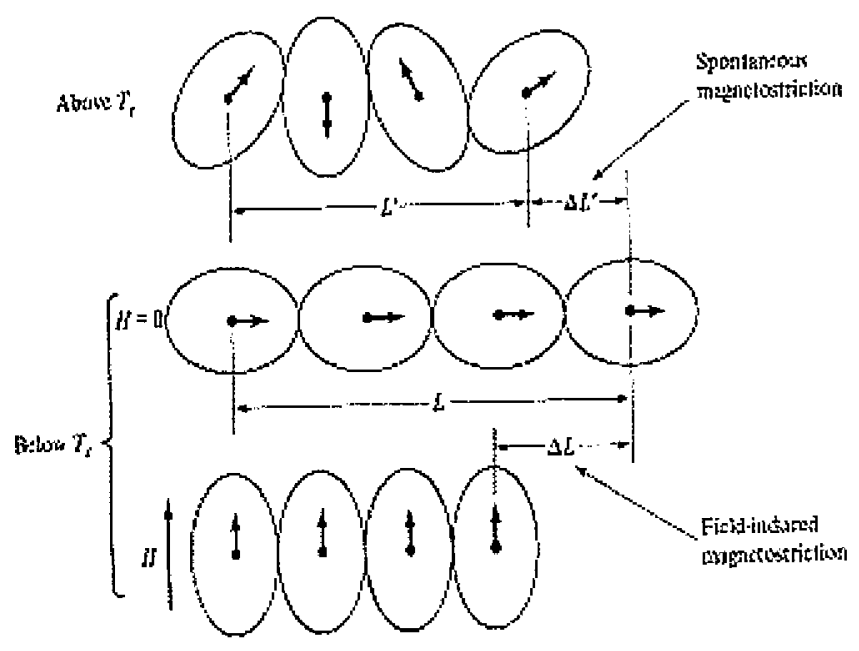

Fig.5-4 Phenomenological description of the Mechanism of magnetostriction (After Jiles, [4])

\section{3 Characterization of magnetostriction curve}

The magnetostriction curve is found to be symmetric with respect to the magnetic field, $M$ direction since $\mathrm{M}$ will be rotated into the $\mathrm{H}$ direction if $\mathrm{H}$ is strong enough.

To understand this characteristics of magnetostriction, here for simplicity, we suppose a 2-D single crystal with single domain magnet is under an external magnetic field applied along y axis, and suppose $\mathrm{x}$ axis is the easy axis, $\mathrm{y}$ is the hard axis, as shown below.

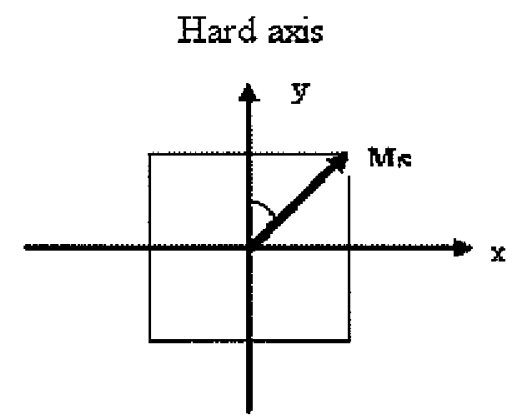

Fig. 5-5 The 2-D magnet with single domain, $\mathrm{x}$ axis is the easy axis, the angle between Ms and $y$ axis is equal to $\theta$. 
Under this circumstance, the total energy for this 2-d magnet is the sum of:

$$
E_{\text {total }}=E_{\text {mag }}+E_{\text {anis }}+E_{\text {mag-elast }}+E_{\text {elast }}+E_{\text {stress }}
$$

where $E_{\operatorname{mag}}$ is the magnetic field energy which is equal to

$$
E_{\operatorname{mag}}=-\vec{M} \cdot \vec{H}=-M H \alpha_{y}
$$

$\alpha_{y}=\cos \theta$.

$E_{\text {anis }}$ is the magnetic anisotropy energy, which is the energy required to rotate the $M$ from its easy axis, and can be expressed as:

$$
E_{\text {anis }}=-K \alpha_{x}
$$

where $\alpha_{x}=\sin \theta$

$E_{\text {mag-elast }}$ is the magnetoelastic coupling energy, if the material is considered isotropic, the energy will have this form:

$$
E_{\text {mag-elast }}=-b\left(\varepsilon_{x x} \alpha_{x}^{2}+\varepsilon_{y y} \alpha_{y}^{2}+\varepsilon_{x y} \alpha_{x} \alpha_{y}\right)
$$

where $b$ is the magnetoelastic coupling constant.

$E_{\text {elast }}$ is the elastic energy, and can be written as:

$$
E_{\text {elast }}=\frac{1}{2} C_{11} \varepsilon_{x x}^{2}+\frac{1}{2} C_{11} \varepsilon_{y y}^{2}+C_{12} \varepsilon_{x x} \varepsilon_{y y}+\frac{1}{2}\left(\frac{C_{11}-C_{12}}{2}\right) \varepsilon_{x y}^{2}
$$

where $\mathrm{C}_{11}, \mathrm{C}_{12}$ is the elastic constant, the notation for the subscript following the rule: $\mathrm{xx}=1$, $y y=2$. 
$E_{\text {stress }}$ is the coupling energy between strain and stress $\sigma$ applied along y axis, can be written as:

$$
E_{\text {stress }}=-\sigma \varepsilon_{y y}
$$

Substituting each energy express into the equation (5-6), and take the derivative with respect to strain. Since we want to find the response of strain to the applied field, we need to make

$$
\begin{aligned}
& \left(\frac{\partial E_{\text {total }}}{\partial \varepsilon_{i j}}\right)=0 \text {, then we get: } \\
& \qquad \begin{array}{c}
b \alpha_{x}^{2}=b\left(\frac{M_{x}}{M_{S}}\right)^{2}=C_{11} \varepsilon_{x x}+C_{12} \varepsilon_{y y} \\
b \alpha_{y}^{2}+\sigma=b\left(\frac{M_{y}}{M_{S}}\right)^{2}+\sigma=C_{11} \varepsilon_{y y}+C_{12} \varepsilon_{x x} \\
b \alpha_{x} \alpha_{y}=b\left(\frac{M_{x}}{M_{S}}\right)\left(\frac{M_{y}}{M_{S}}\right)=\frac{1}{2}\left(C_{11}-C_{12}\right) \varepsilon_{x y}
\end{array}
\end{aligned}
$$

where $\mathrm{M}_{\mathrm{x}}, \mathrm{M}_{\mathrm{y}}$ is the component of magnetization along $\mathrm{x}, \mathrm{y}$ axis. From above equations, it is clear that the magnetostriction $\varepsilon$ is a function of $\mathrm{M}^{2}$. Therefore, the typical magnetostriction curve has the following shape with regard to the magnetization curve. 


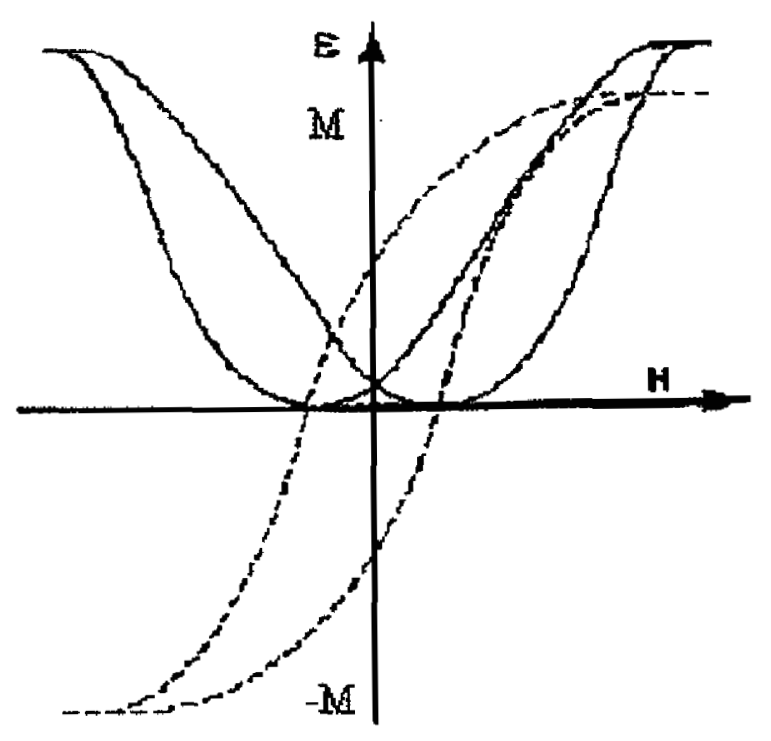

Fig. 5-6 The relationship between the magnetization curve and the magnetostriction curve. $\mathrm{M}-\mathrm{H}$ curve is odd, $\varepsilon-\mathrm{H}$ is even with respect to the applied magnetic field $[2,28]$.

In the discussion above, we have mentioned two terms to characterize the magnetostriction response to the applied field: magnetostriction, and magnetostriction constant. Here we clarify the difference between them. When we talk about magnetostriction in this thesis, we actually refer to the Joule magnetostriction, which is the change in length as a response to the applied field. However magnetostriction constant is defined as the change in length measured along $\mathrm{M}$ direction at saturation.

\section{(2) Anisotropic Magnetostriction}

It is found that the magnetostriction value and sign along different crystallographic directions are quite different in iron single crystal [28], as shown below. 


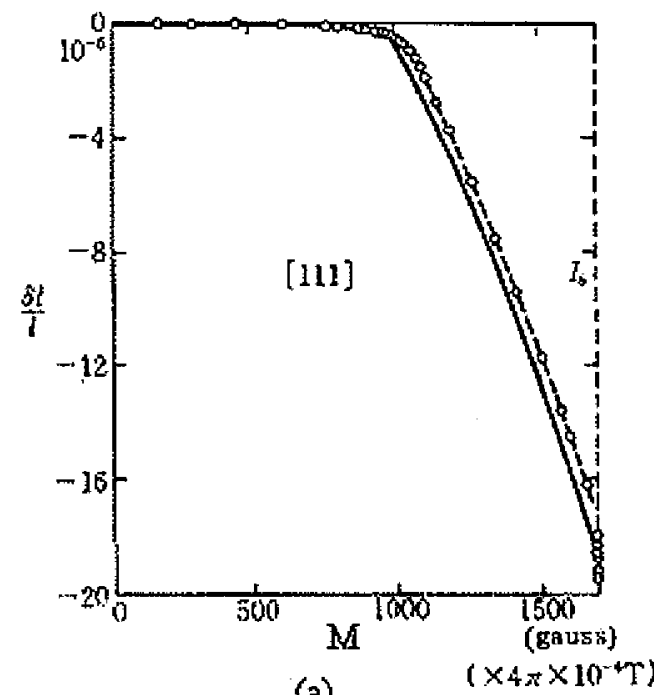

(a)

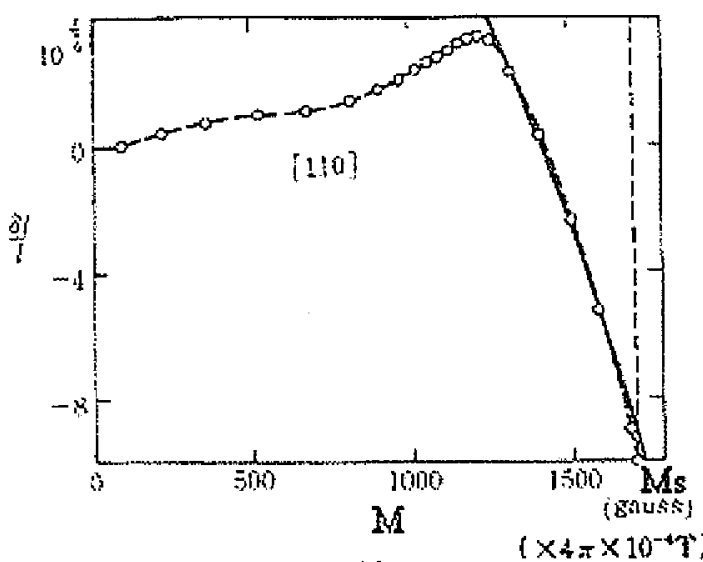

(b)

Fig.5-7 Magnetostriction of iron single crystal as function of M/Ms along different direction (a) along [111]; (b) along [110] (after Chikazumi [28]).

For a single crystal sample, during the technical magnetization process, firstly, it is magnetized by domain wall displacement, and then the magnetization process is completed by magnetization rotation in the domains. When magnetic field is applied along different axes, the domain configuration and displacement are different, such as along [111], first the domains configuration is reduced into those domains with their magnetization directions along the easy axes [100], [010], [001] by wiping out the $180^{\circ}$ domain walls, by this mechanism, the net magnetization is increased. However, $180^{\circ}$ domain wall displacement can not produce magnetostriction. Further increasing magnetic field, $M$ is increased by rotating the magnetization in each domain into the field direction, and magnetostriction only then is observed. If $\mathrm{H}$ is applied along the [110] direction, the remaining domains before magnetization rotation start are those domains with $M$ parallel to [100] or [010] direction. The phenomenon that magnetostriction value and sign depends on the crystallographic direction of magnetization is called anisotropic magnetostriction. 
For a randomly oriented polycrystal, the magnetostriction is isotropic if no stress is applied on it, and there is no textural structure in it.

The sample shape also has an effect on the magnetostriction. This effect is called the "form effect". However, usually it is very small [29], as shown in the following figure for an iron sample with different dimension rations: $\mathbf{k}=\mathbf{l} / \mathbf{d}$, where $l$ is the length, $d$ is the diameter for ellipsoid shape sample.

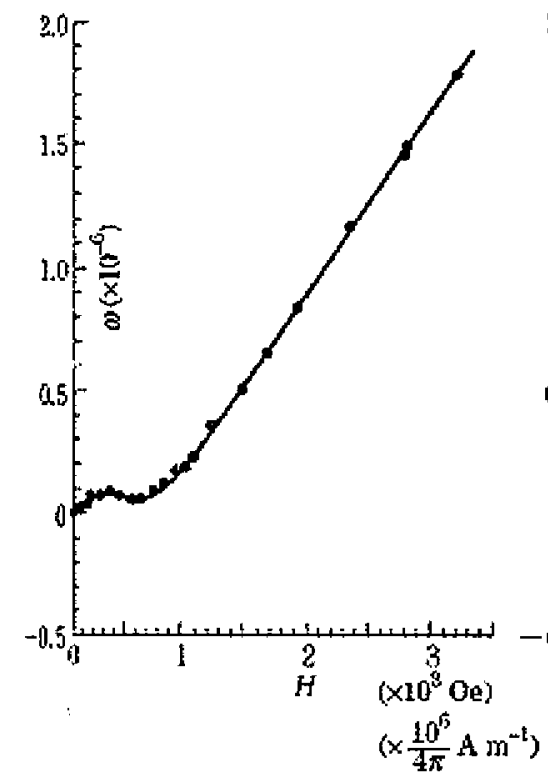

(a)

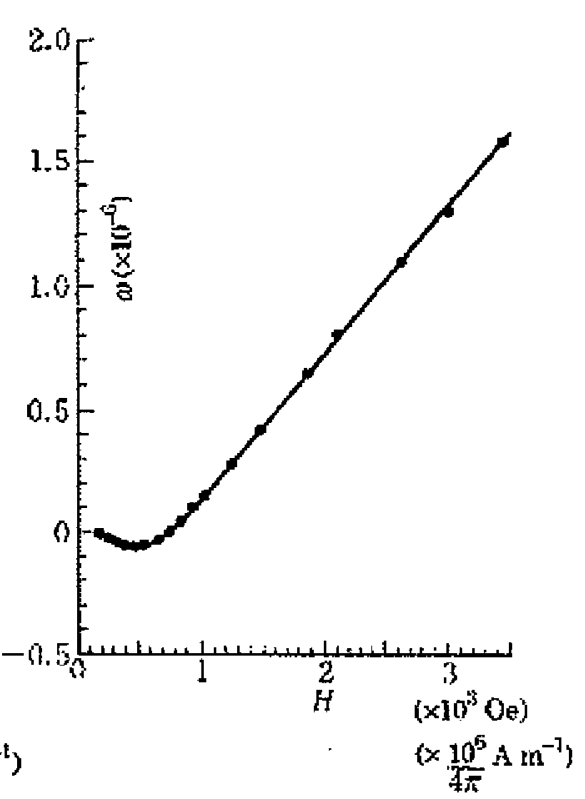

(b)

Fig.5-8 The effect of iron sample shape on the magnetostriction with different dimensional ratios: (a) $\mathrm{k}=16.9$ (b) $\mathrm{k}=41$ 1.6. (After Kornetzki [29] from Chikazumi's book: "Physics of Ferromagnetism").

\subsection{Temperature dependence of magnetostriction}

As pointed out before, magnetostriction is not only a function of composition, magnetic field, stress, crystallographic direction, sample shape, but also is a function of temperature. This is because the magnetoelastic coupling coefficient $b$ varies with temperature. Callen et al [30]. 
have developed a model based on the localized moment assumption, and shown that the magnetoelastic coefficient $b^{\gamma, 2}(T)$ and $b^{\varepsilon, 2}(T)$ vary as the spontaneous magnetization of material is reduced. The following figure shows magnetostriction constant change with temperature for Gd metal.

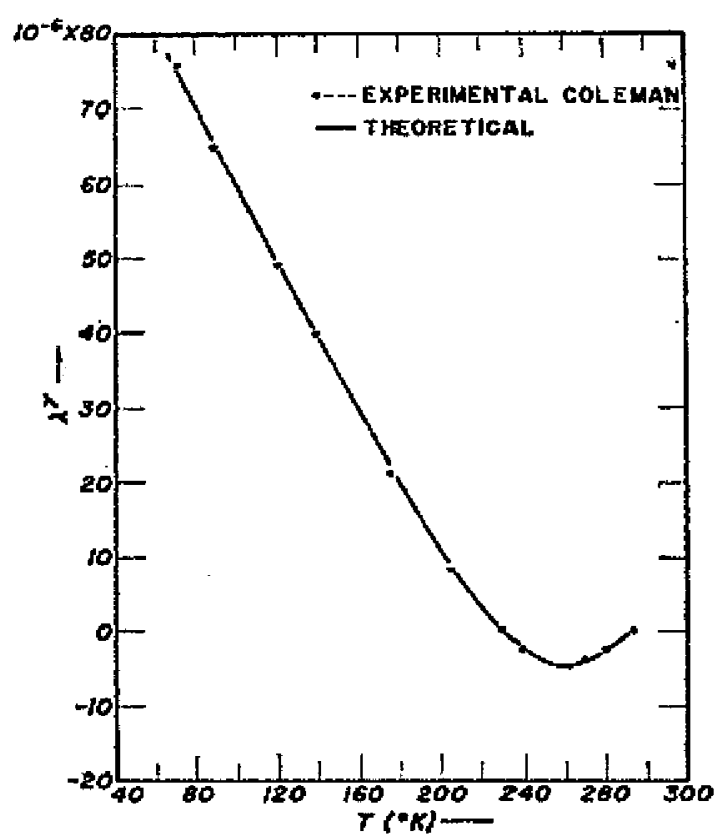

Fig.5-9 Temperature dependence of magnetostriction constant of Gd (After E. Callen [30])

\subsection{RKKY exchange and superexchange coupling}

As mentioned in the previous chapter, for the $\mathrm{Gd}_{5}\left(\mathrm{Si}_{\mathrm{x}} \mathrm{Ge}_{1-\mathrm{x}}\right)_{4}$ system, it is thought that the RKKY exchange and superexchange coupling that are responsible for magnetic ordering.

RKKY exchange is believed to take responsibility for the magnetic ordering for rare earth metals and their compounds. For these pure metals, there are 3 electrons in their outer shell, these electrons make no contribution to atomic magnetic moment, but serve as a medium for exchange interaction which produces the ordered magnetic structure. These 3 electrons also 
contribute to the conductivity and metallic bonding. The magnetic moments comes from the unpaired $4 \mathrm{f}$ electrons which are inside the shell. For example in $\mathrm{Gd}$, the orbital moment $\mathrm{L}$ is equal to 0 , the magnetic moments comes from the contribution of spin moment of 7 unpaired electrons, $\mathrm{S}=\mathrm{J}=7 / 2$, resulting in $\mathrm{m}=7.9 \mu_{\mathrm{B}}$.

The RKKY exchange for the helimagnetic structure is believed due to the oscillatory polarization of 3 conduction electrons [28] in the outer shell. The polarization can be described by a function:

$$
\sigma(r) \propto S \times f\left(2 k_{F} r\right)
$$

where $s$ is the spin, $k_{F}$ is the wave vector at the Fermi surface, $r$ is the distance of spins. The $f$ is a damping function which is very sensitive to the distance $r$, and can be expressed as:

$$
f(x)=\frac{1}{x}(x \cos x-\sin x)
$$

The oscillatory behavior of $\mathrm{f}(\mathrm{x})$ can be drawn as:

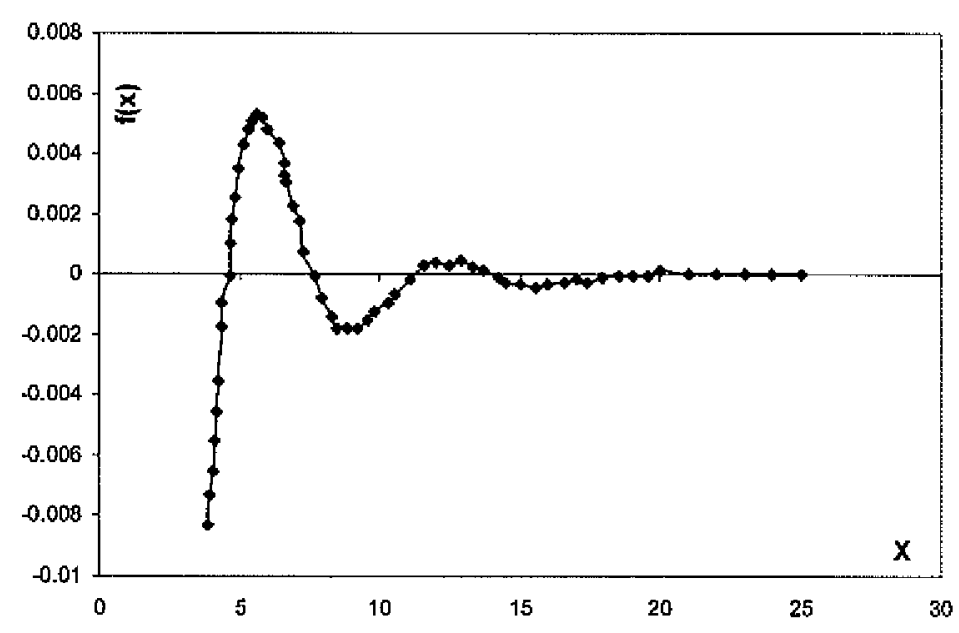

Fig .5-10 Damping characteristics of $\mathrm{f}(\mathrm{x})$ function for RKKY interaction (after Chikazumi $[28]$ 
Therefore, from the above discussion, it is clear that the RKKY interaction is sensitive to the atomic distance and the conduction electron distribution. For $\mathrm{Gd}_{5}\left(\mathrm{Si}_{\mathrm{x}} \mathrm{Ge}_{1-\mathrm{x}}\right)_{4}$, during the heating and cooling process, the crystallographic phase transformation and associated bond breaking and reforming between the slabs, greatly changes the atomic distance and conduction electron distributions, and results in a simultaneous magnetic ordering and structural transformation.

As for superexchange coupling, it is believed that an indirect exchange interaction occurs between magnetic atoms through the nonmagnetic atoms $(\mathrm{Si})-(\mathrm{Si})$ or $(\mathrm{Si})-(\mathrm{Ge})$. This type of exchange is sensitive to the nonmagnetic atoms, and sensitive to the angle between the M1$\mathrm{O}-\mathrm{M} 2$, where $\mathrm{M} 1, \mathrm{M} 2$ represent the magnetic atoms, $\mathrm{O}$ represents nonmagnetic atoms. For example, for $\mathrm{MnO}$ on which this theory is first developed by Shull and Smart [31], the superexchange coupling is increased in the order of $\mathrm{O}^{2-}, \mathrm{S}^{2-}, \mathrm{Se}^{2-}$. And it is found that the superexchange is strongest when the angle M1-O-M2 is $180^{\circ}$. When a crystallographic phase change occurs, the crystal structure changes, not only the distance between magnetic atoms changes, but also the angle between them will be changed, therefore, it maybe expected that the superexchange will also be affected. 


\section{Chapter Six}

\section{Experimental Details}

\subsection{Closed-Cycle Refrigerator System}

Since most of the interesting behavior of the samples used in this study is below ambient temperature, we used a closed-cycle refrigeration system to achieve the necessary low temperatures. The system schematic is shown in Fig.6-1 (a).

The commercial closed cycle refrigerator (CCR) system can provide low temperature environments (from $10 \mathrm{~K}$ to $325 \mathrm{~K}$ ) for the thermal expansion and magnetostriction measurements reported in this thesis. Closed cycle systems recirculate a fixed volume of gas to provide cooling to the sample mounting stages, and no gas is added or removed during operation. The CCR utilizes the Gifford-McMahon thermodynamic cycle, based on the controlled compression and expansion of helium gas. A compressor is used to provide the high pressure helium gas needed for the cycle. Flexible metal gas lines deliver the compressed helium gas to the refrigerator, and return the low pressure gas to the compressor for recirculation. The refrigerator includes two cold stages, one for cooling the sample and one for cooling a radiation shield that surrounds the sample. The system is shown in Fig. 61(b). 


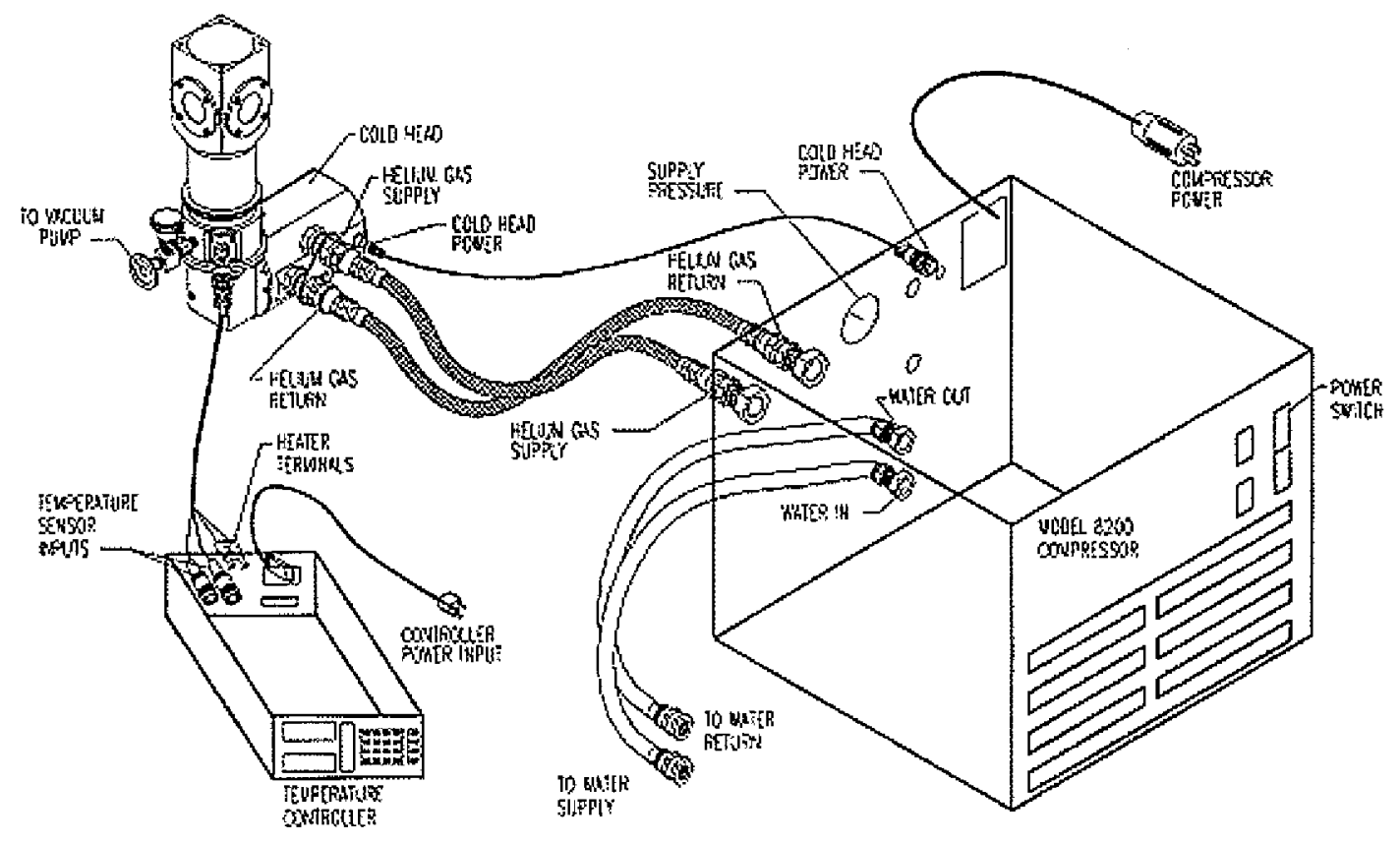

Fig. 6-1(a) Interconnection of Janis CCR System (Courtesy by CTI-Cryogenics Helix Tech Corp.)

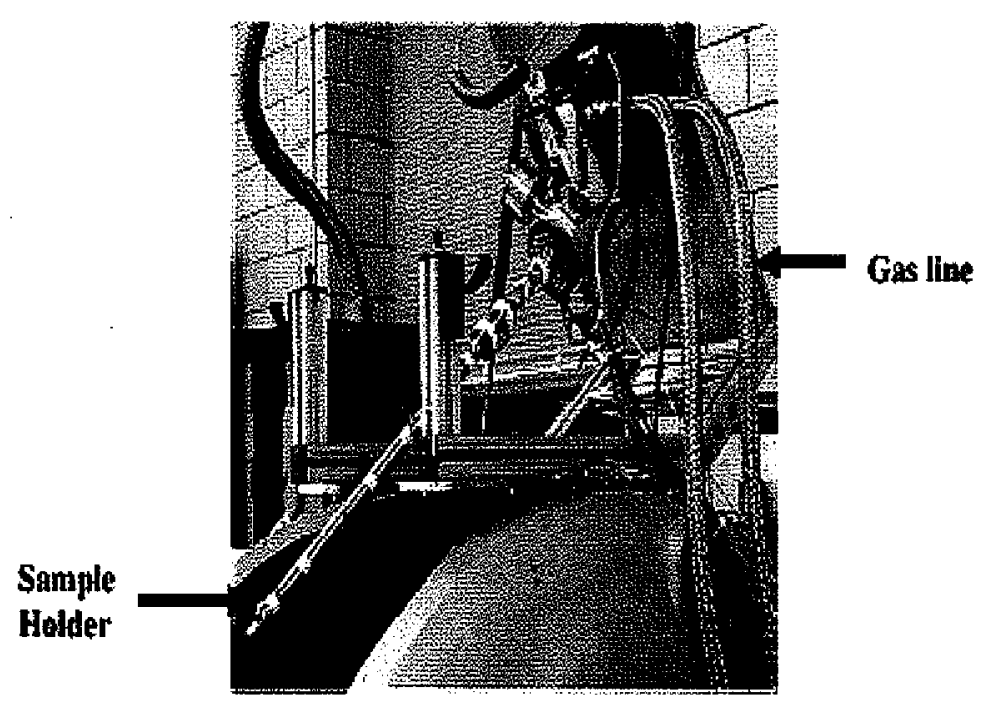

Fig. 6-1 (b) The Janis closed cycle refrigerator system (CCR) 
The measurement system for thermal expansion and magnetostriction measurements is shown in Fig.6.2 . Detailed operational procedure is enclosed in Appendix A.

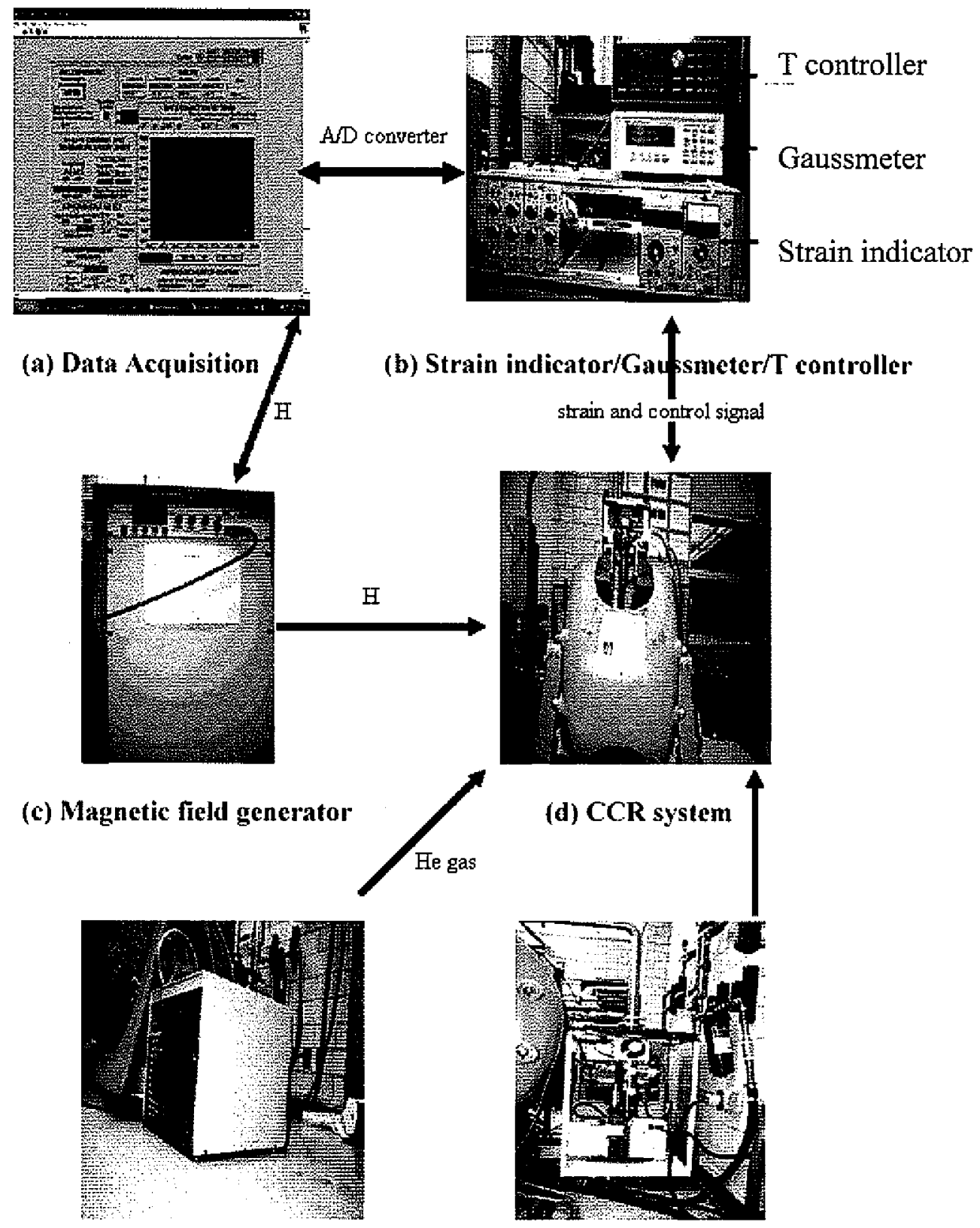

(c) Compressor

(f) Vacuum system

Fig. 6-2 Measurement system for low temperature thermal expansion and magnetostriction 


\subsection{Thermal expansion}

In this thesis, the thermal expansion was measured by using the strain gauge method. Fig. 6-3 shows how the strain gauge was mounted on the sample. As shown, the surface where the strain gauge was mounted should be perpendicular to the surface which is mounted on the sample holder, leaving the measurement direction free to expand and contract. In Appendix B, a detail sample mounting procedure is given. Magnetic field can be applied along the measurement direction, and can be reversed by changing the dc current direction. The sample can also be rotated to make an angle between the strain measurement direction and magnetic field.
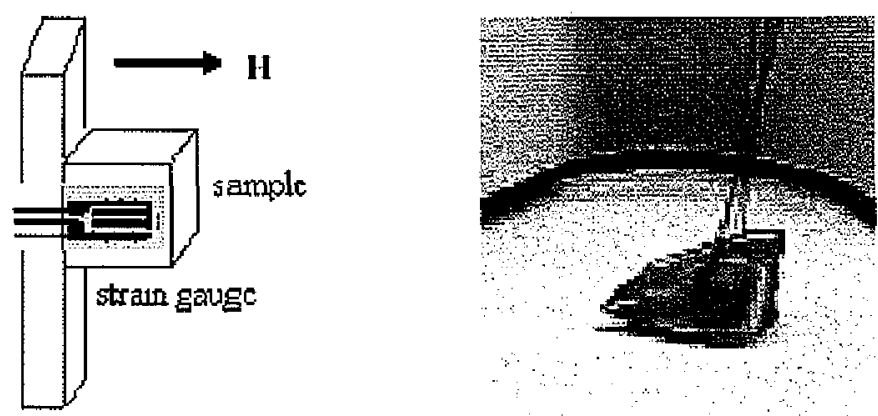

Fig.6-3 sample mounting schematic and actual sample with strain gauge

Fig. 6-4 shows how data were collected in the software "Cryostat Controller" written in LabView $^{\odot}$. In this user interface, we can choose which channel to use for the measurement (for model 3800 wide strain gauge indicator, the gauge factor is set in the front panel. The detailed operational procedure will be discussed later), and use which sensor to use for temperature measurement. The strain gauge factor, heater power, and temperature upper limit can be set by the user. For these studies, each data point is acquired either when the temperature changes by 1 degree or strain changes by $4 \mathrm{ppm}$. 


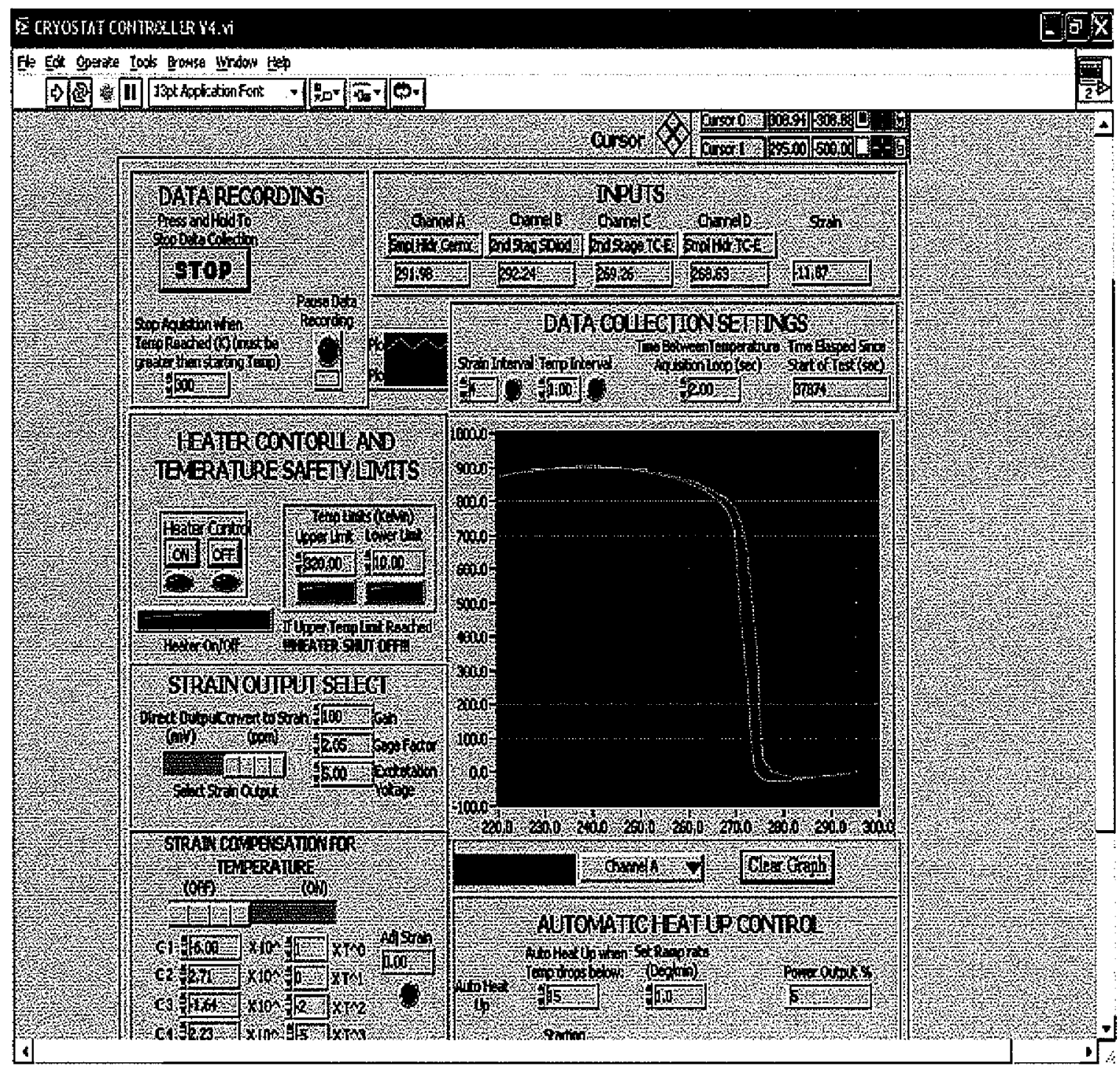

Fig.6-4 Thermal expansion measurement software user interface

Fig. 6-5 shows the measurement system with strain gage indicator (VISHAY Micromeasurement Group, Model 2130), Gaussmeter (Lakeshore ${ }^{\circledast}$ Model 4500) for magnetic field measurement, and temperature controller (CRYO.CON ${ }^{\circledR}$, Cryogenic temperature controller, Model 34). In the temperature controller, accurate temperature 
control is realized by adopting PID (Proportion, Integration, and Differentiation) algorithm. There are four sensors used for four channels in the Janis CCR system, as follows:

Channel A: Cernox sensor at the sample holder for measuring the sample temperature, its working range is $4 \sim 325 \mathrm{~K}$.

Channel B: Silicon diode sensor at the refrigerator second state, its working range is 1.4 $\sim 325 \mathrm{~K}$;

Channel C: Thermocouple at the sample holder for $\mathrm{T}>325 \mathrm{~K}$ measurement.

Channel D: Thermocouple at the higher temperature stage, we leave it idle in this CCR system;

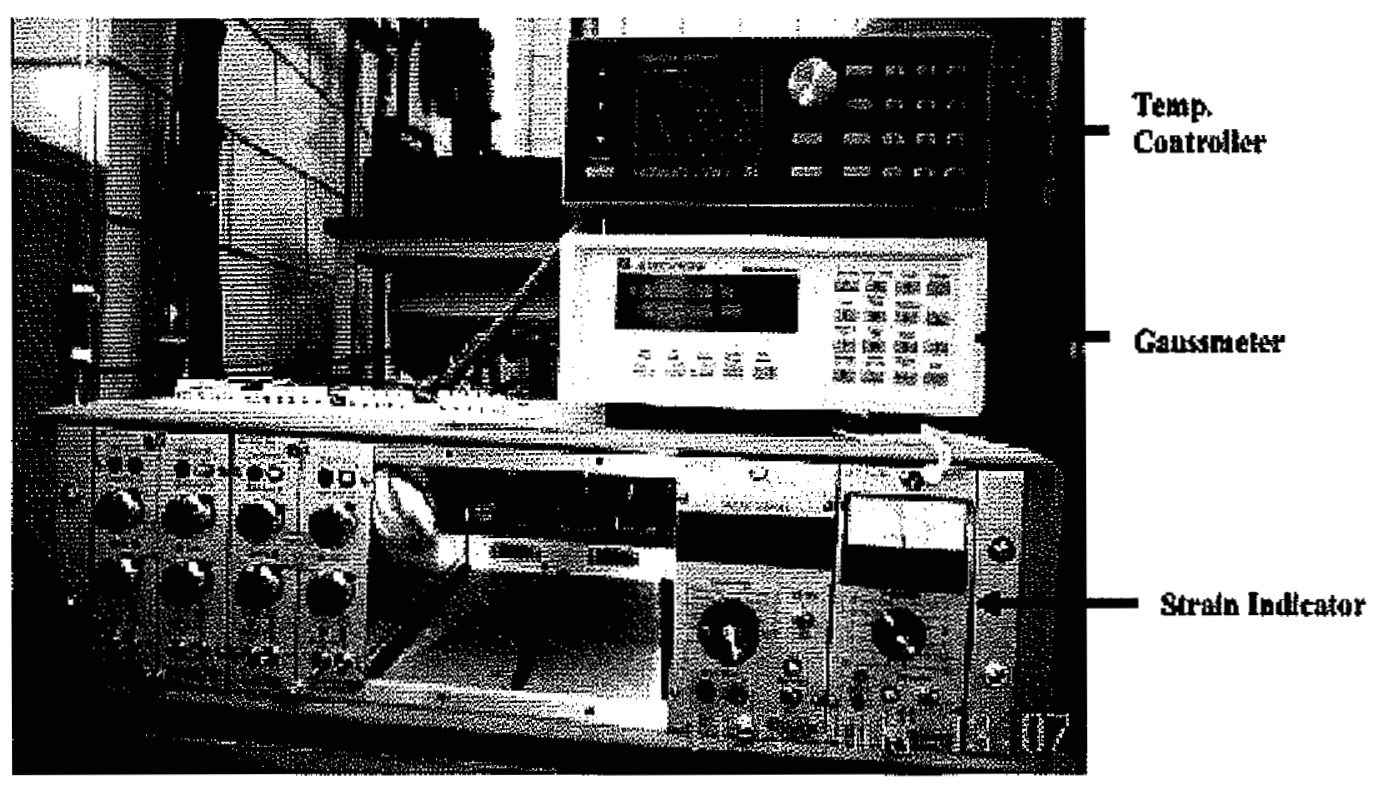

Fig. 6-5 Strain indicator, gaussmeter, and temperature controller

Beside the strain gauge indicator (VISHAY ${ }^{\circledR}$ 2130) discussed above, we also used another type strain gauge indicator in our measurements: VISHAY ${ }^{\circledR 3} \mathbf{3 8 0 0}$ wide range strain gauge indicator, as shown below. The 2130 can measure four channels simultaneously, but needs to 
translate the measured voltage signals into the strain by the software. The latter, 3800 , however, can directly measure the strain if properly calibrated, and can only measure one channel. Detailed procedure for calibration is given in Appendix $\mathbf{C}$. The wiring between strain gauge and indicator poles are shown in Fig. 6-6.

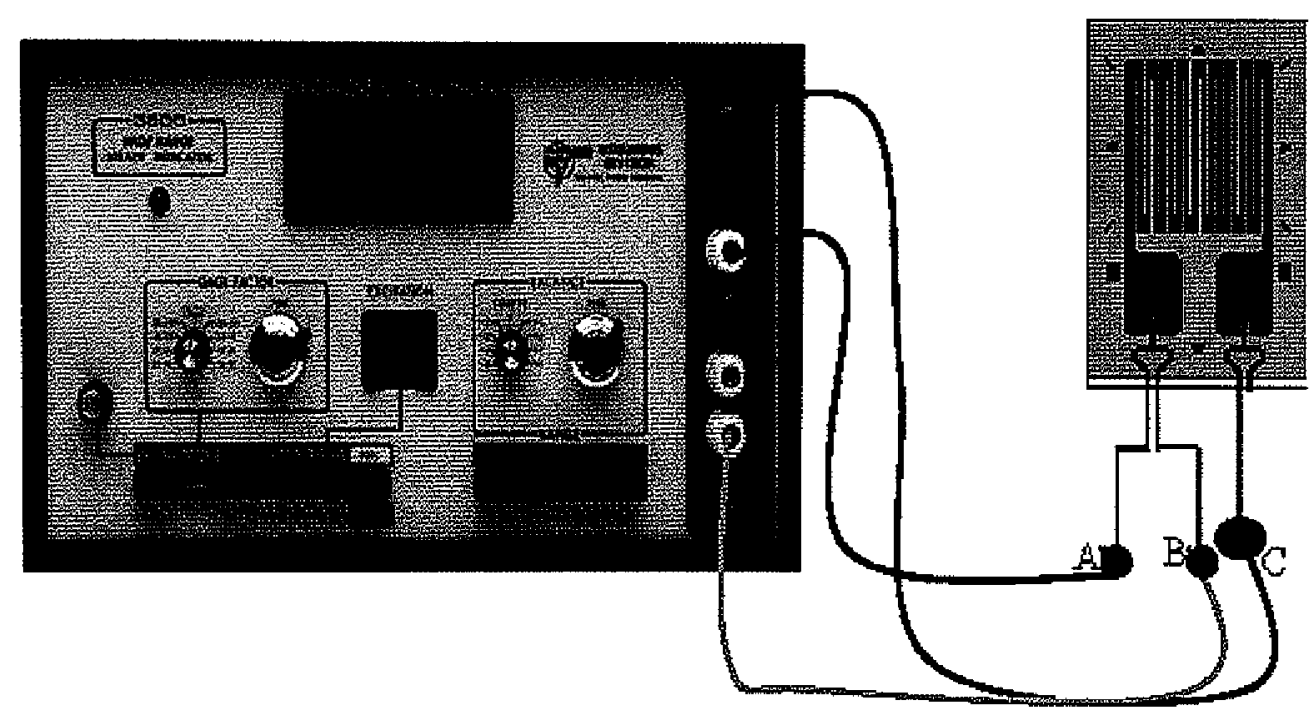

Fig. 6-6 Wiring schematic: $\mathrm{R}_{\mathrm{AB}}=0.6 \Omega, \mathrm{R}_{\mathrm{AC}}=350 \Omega, \mathrm{R}_{\mathrm{BC}}=350 \Omega$

\subsection{Magnetostriction Measurements}

Magnetostriction measurements use the same techniques as the thermal expansion, except that magnetostriction was carried out at a constant temperature and a varying magnetic field, whereas thermal expansion was carried out at constant field and varying temperature. Fig. 67 shows the user interface of MS measurement software. In this software, we can set up the field increment, the time waiting for the field to be stable, the maximum field, Hall sensor parameter, gauge factor, and excitation voltage. 


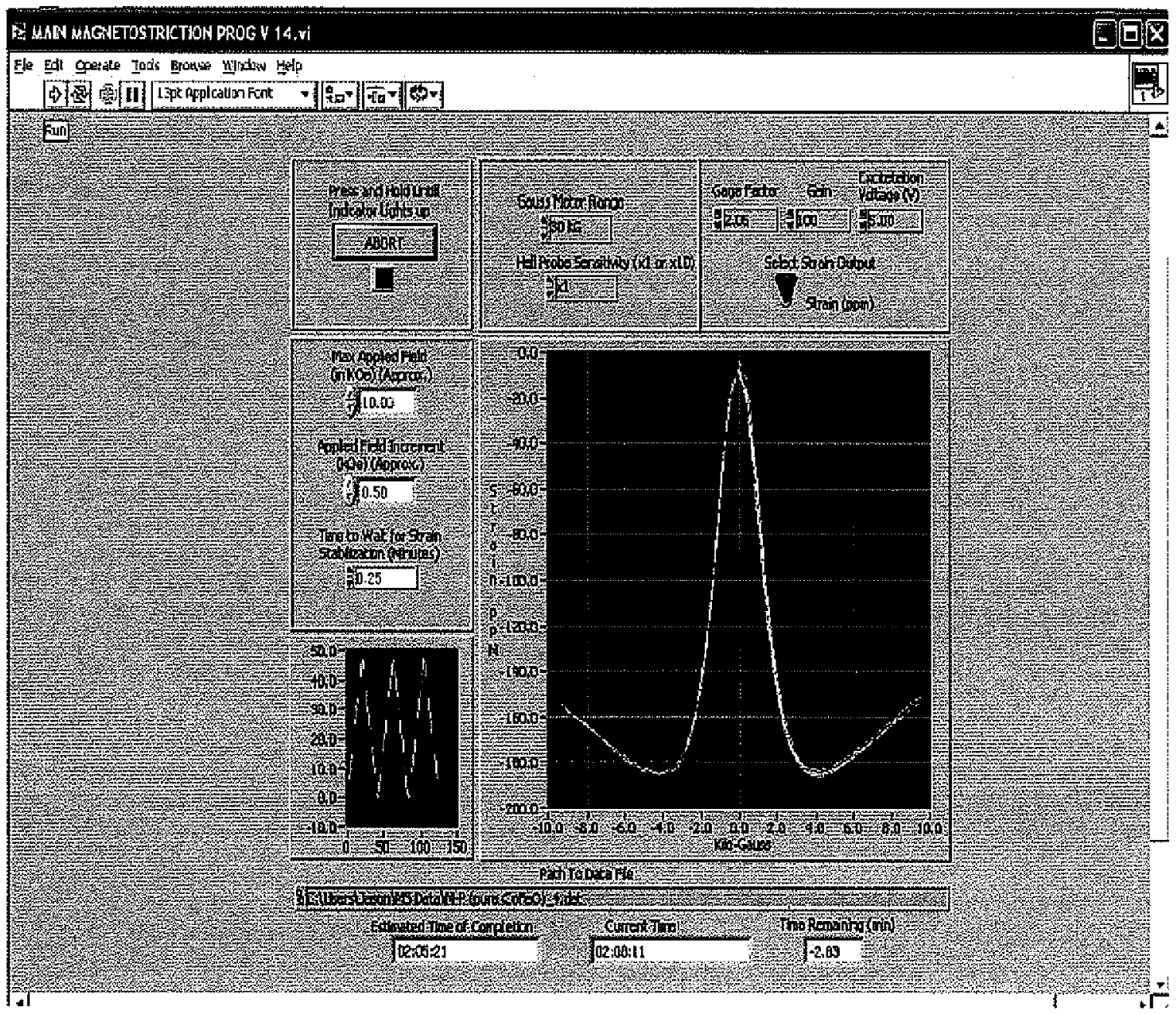

Fig. 6-5 Magnetostriction Measurement software

\subsection{Measurement Techniques}

From the definition of thermal expansion coefficient, we know that determination of the thermal expansion coefficient $\alpha$ needs measurement of two physical quantities: strain and temperature. Ref. [27] has compared several common used methods for $\alpha$ measurements: Mechanical dilatometry, optical methods, strain gauge methods, diffraction techniques. In this thesis, we use the strain gauge method. 


\section{(1) Strain Gauge Method}

The strain gauge used in this study is the bonded metallic strain gauge, which has the characteristic that its electrical resistance changes in proportion to the change of strain in the strain gauge, which is bonded properly on the surface of the studied sample. When the sample changes its length on cooling/heating, or changed by magnetic field, the strain gauge also changes its shape. Therefore, the strain gauge mounting is the most important step in thermal expansion and magnetostriction measurements, which determines the experimental errors. Detailed strain gage installation procedure can be found in the website of MicroMeasurement Inc. [46].

The metallic strain gage looks like the picture shown in the following figure (Fig. 6-8), which consists of a very fine wire arranged in a grid pattern, flexible support which should be boned on the sample surface, and four wires coming out. Strain gauges used (type WK-06-031CF350 ) in this study is the one with the following specifications: Resistance $=350 \Omega \pm 0.4 \%$, Gage factor $=2.05 \pm 1.0 \%$.

Active grid

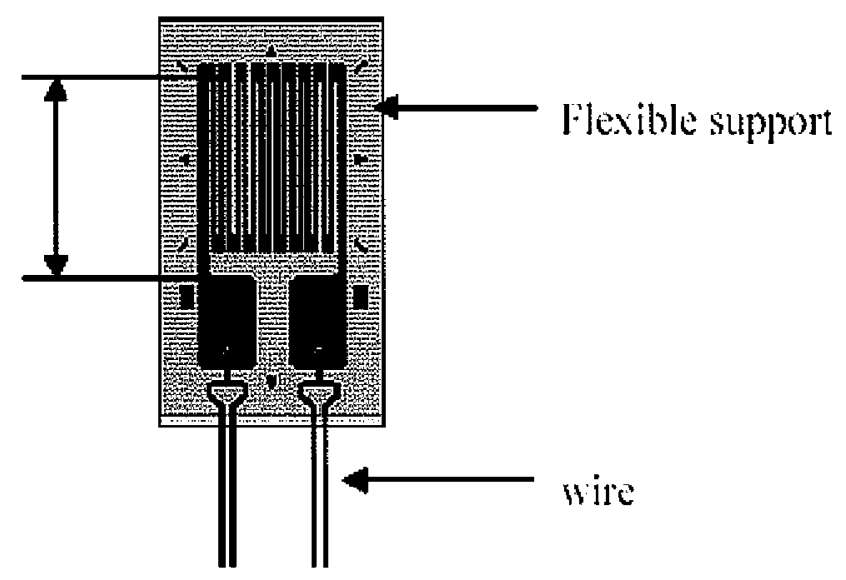

Fig. 6-8 WK-Series Gages manufactured by Micro-Measurements Division (Courtesy of Micro-Measurements Inc.) 
Gage factor is a very important specification in strain gage measurement. It is defined as follows: It is the ratio of fractional change in electrical resistance to the fractional change in length (strain):

$$
G F=\frac{\Delta R / R}{\Delta L / L}=\frac{\Delta R / R}{\varepsilon}
$$

the gauge factor for this study is about 2.05 or 2.04 depending on which type is used. There are four wires coming out from the strain gage, but we only use three of them, leaving one idle in the quarter bridge circuit. The resistance $350 \Omega$ is also important specification, which will be used to test whether the strain gage working well and to connect the strain gage wire with the strain gage indicator, this was shown clearly in Fig. 6-6.

\section{(2) Strain Gauge Measurement circuits}

The basic principle of strain gage measurement is based on the Wheatstone bridge circuit, as shown in Fig. 6-9. It consists of four so-called resistive arms (R1, R2, R3, R4) with an excitation voltage source $V_{E X}$.

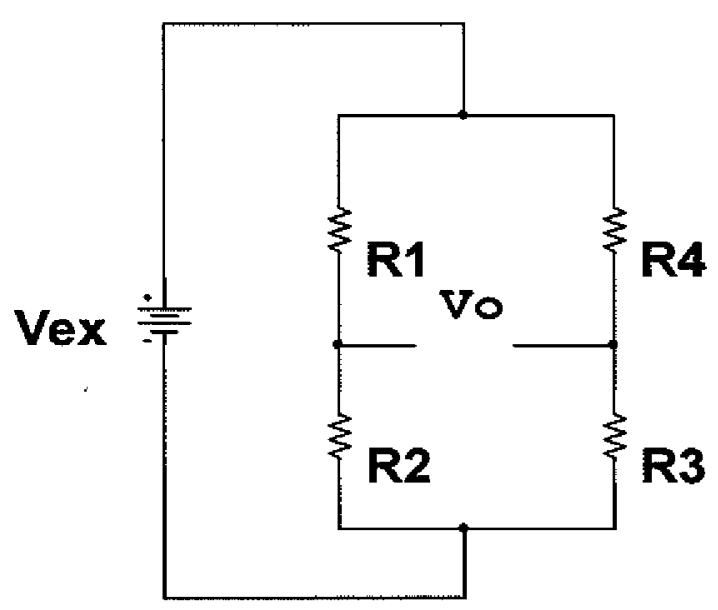

Fig.6-9 Wheatstone Bridge 
The output voltage $\mathrm{V}_{0}$, is found to be:

$$
V_{0}=\left[\frac{R_{3}}{R_{3}+R_{4}}-\frac{R_{2}}{R_{1}+R_{2}}\right] \cdot V_{e x}
$$

If any arm $\left(R_{1}, R_{2}, R_{3}, R_{4}\right)$ changes its resistance linearly then the measured out-of-balance voltage $\mathrm{V}_{0}$ will change in proportion to the resistance change. There is a special case: if $R_{1} / R_{2}=R_{4} / R_{3}, V_{0}$ is equal to 0 . If this occurs, we call the circuits balanced.

If we replace $R_{4}$ in Fig.6-9 with an active strain gauge as shown in Fig.6-10 (a) and balanced by adjusting the resistance of $\mathrm{R} 1, \mathrm{R} 2$ and $\mathrm{R} 3$, because the strain gage is bonded on the studied sample, any factor, such as temperature in thermal expansion measurement, which changes the sample shape, will result in a change in the resistance of strain gage boned onto it. The balance will be broken, and a non-zero voltage resulting in the output voltage, which is input into the data acquisition software. If the nominal resistance of the strain gauge is designated as $R_{G}$, in our case, it is $350 \Omega$, according to the definition of gage factor, the change resistance due to the change in sample shape, the $\Delta R=R_{G} \cdot G F \cdot \varepsilon$. If we assume $R_{1}=$ $R_{2}$ and $R_{3}=R_{G}$, the relationship between the $V_{0}$ and strain $\varepsilon$ can be expressed as the equation in 6-3.

The simulation of equation $6-3$ is shown in the Fig. 6-10 (b). It is clear that $V_{0}$ is linear with the strain $\varepsilon$. 


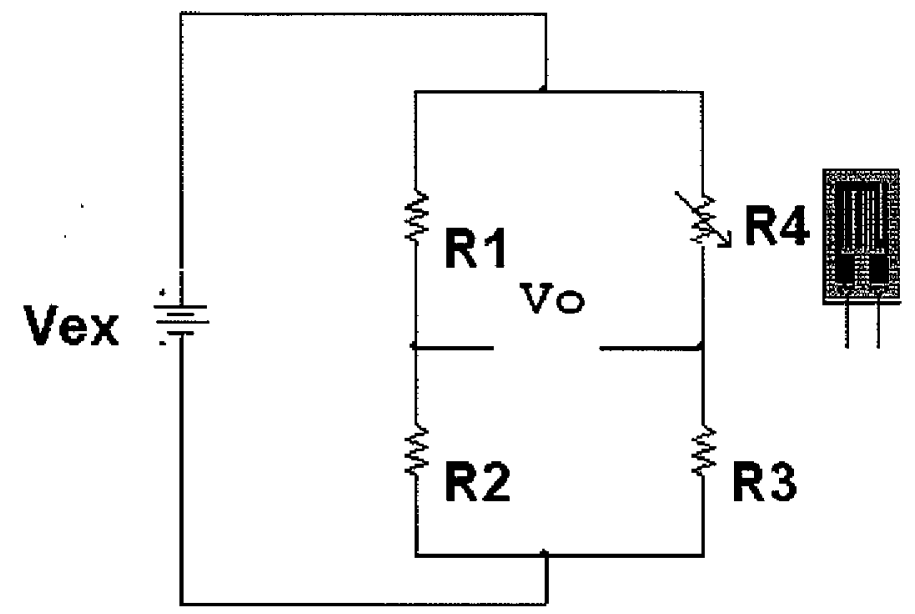

$$
V_{0}=\frac{V_{c x}}{2+G F \times \varepsilon}-\frac{V_{c x}}{2}
$$

Fig. 6-10 (a) Quarter-Bridge Circuit

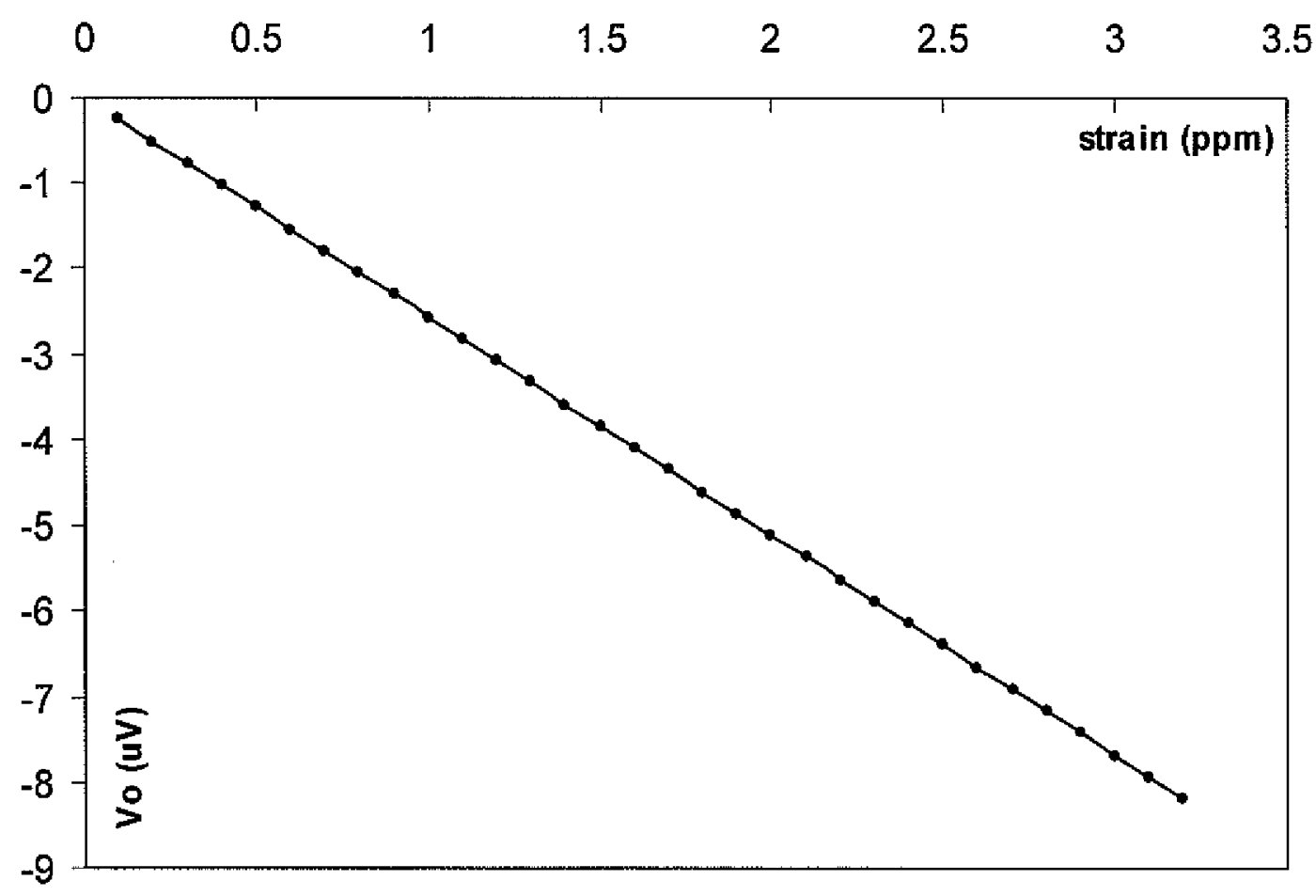

Fig. 6-10 (b) Simulation of equation (6-3) 


\subsection{Fundamentals of Magnetic Force Microscopy (MFM)}

The magnetic force microscopy which was first reported by Martin and Wickramasinghe [32] was developed from the earlier atomic force microscope of Binnig, Quayte and Gerber [33] in which the alternating voltage on the probe tip is replaced with an alternating magnetic field. The device samples the magnetic field gradients close to the surface of specimen. The method is therefore basically a scanning force probe technique based on magnetic forces between the magnetized tip of the microscope and specimen, as shown in Fig. 6-11.

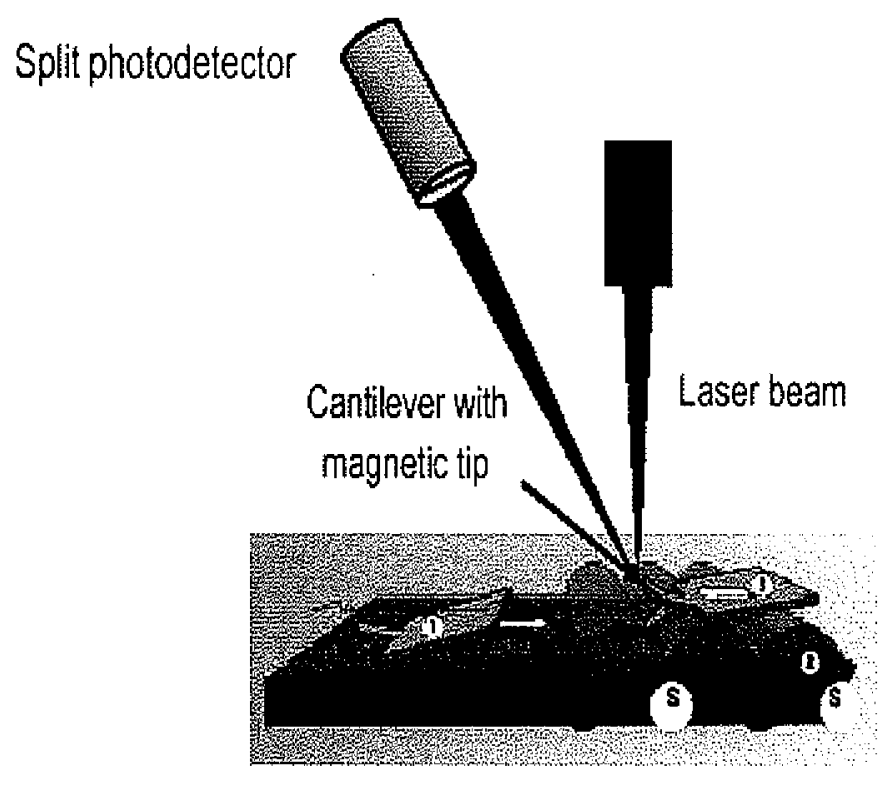

Fig. 6-11 Magnetic imaging using lift mode. For each scan line, the surface topography is first determined with Tapping Mode, in which the tip lightly taps the sample (1) shown in the figure. The tip is then raised to the lift height (2), and a second pass is made to acquire magnetic force data (3) while keeping the tip-to-sample spacing constant (Courtesy of Digital Instrument Co.).

In this thesis, a magnetic force microscopy manufactured by Digital Instrument Inc. is used to image the magnetic samples. Fig. 6 shows how it is used to detect magnetic field variation on the surface of magnetic material. There are two main modes of operation: constant height 
and variable height. In the first mode, it operates by scanning the tip attached to the end of an oscillating cantilever across the sample surface. A laser light from a solid state diode is reflected off the back of the cantilever and collected by a position sensitive detector (PSD) consisting of two closely spaced photodiodes whose output signal is collected by a differential amplifier. Angular displacement of cantilever results in one photodiode collecting more light than the other photodiode producing an output signal (the difference between the photodiode signals normalized by their sum) which is proportional to the deflection of the cantilever. The change in phase angle of vibration of the tip can be related to the magnetic force gradient at the surface. The typical scanning height in this mode of operation is $100 \mathrm{~nm}$. The second mode relies on moving the tip closer to the surface by controlling the tip-sample spacing. This can be used to image static magnetic fields. The magnetization in the tip is modulated and the force on the tip is detected in the same way as before, using the laser interferometer to measure vibration amplitude, phase and frequency of the tip. The image formed as result of magnetic force microscopy is obtained by measuring the force or the force gradient, and these images can be used to study magnetic structures such as domain walls, closure domains, Bloch lines and, in the case of magnetic recording media, intentionally magnetized regions of a material. In fact today, it is in the area of magnetic recording media that the MFM is finding the most applications. 


\subsection{Vibrating Sample Magnetometer (VSM)}

In this thesis, $\mathbf{M}-\mathbf{H}$ hysteresis loop is measured by Vibrating Sample Magnetometer, Model 4500, EG\&G Princeton Applied Research, shown in Fig. 6-12.

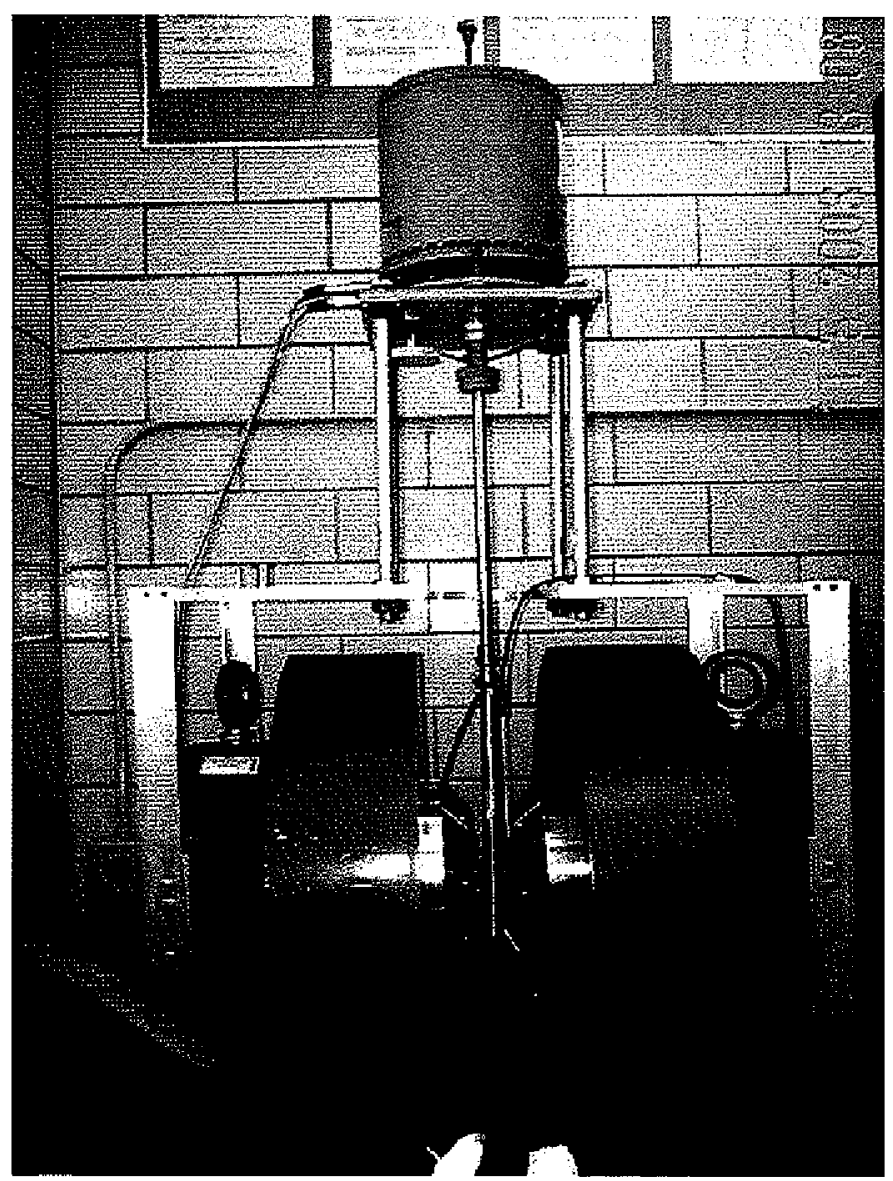

Fig. 6-12 the Model 4500 Vibrating Sample Magnetometer

The basic principle of how this equipment works is easily understood, detailed descriptions were given in ref $[2,4]$. The sample, usually a sphere, small disc or cubic, was glued to the end of the rod. The other end of rod was screwed into a mechanical vibrator. Before the 
measurement, the sample should be centered between two pole pieces by using the three screws to center the sample between two pole pieces: We then (1) adjusted the "UP/Down" screw to maximize the measured moment; (2) adjusted the "Front/Back" screw to maximize the measured moment; (3) adjusted the "Left/Right" screw to minimize the measured moment.

The rod was positioned between the pole pieces of large electromagnet (by Magnion Inc.,). A set of pickup coils was fixed against the pole pieces. The sample rod was fastened to an electric motor, which vibrates the sample up and down in small oscillations. When the electromagnet was energized, it magnetized the sample. The fringing magnetic field produced by the vibrating magnetized sample will result in a time-varying magnetic flux, and be sensed by the pickup coils. The magnitude of induced e. $\mathrm{m}$. $\mathrm{f}$ signals depends on the total magnetic moment of the sample. In the process of measuring the hysteresis loops, the magnetic field firstly was swept at a low rate from maximum positive, then through zero to maximum negative and back again, the magnetization is recorded continuously by the VSM controlled and displayed in the software interface, and the applied field was measured by a Hall-effect gaussmeter. For the M-T measurement, a constant small magnetic field was applied during the heating process. For the $\mathrm{M}-\mathrm{H}$ hysteresis loop measured at low temperature (the capacity of low temperature measurement is 20 degree below the room temperature), a coolant of mixture of dry ice and ethanol was circuited around the sample holder. The temperature was controlled by the Lakeshore temperature controller 3400 . 


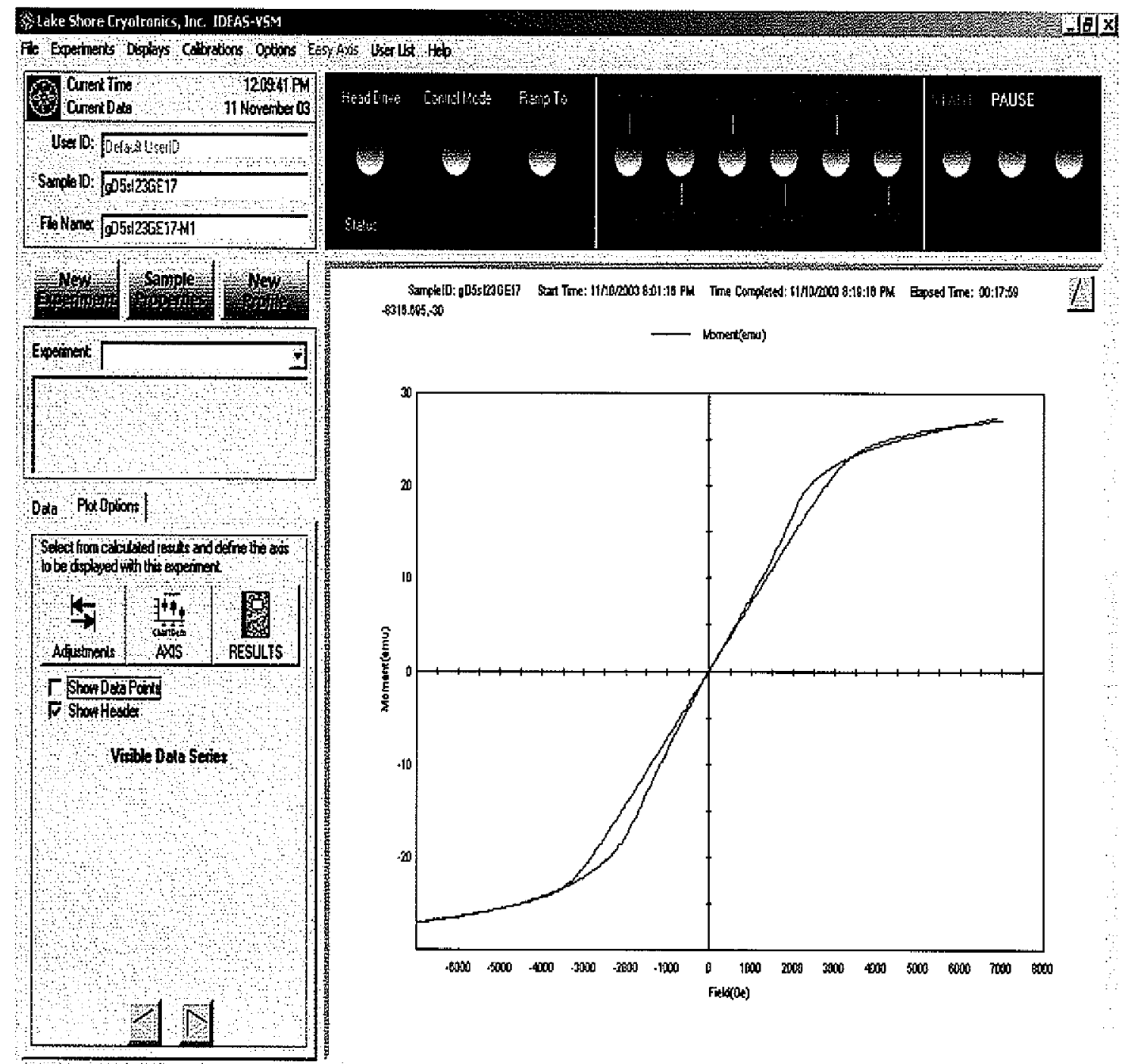

Fig.6-13 Software User Interface for VSM

\subsection{Measurement of magnetic field}

While conducting magnetostriction measurements and thermal expansion measurements in the closed-cycle refrigeration system, applied magnetic field needs to be measured. In these 
experiments, a Lake Shore Model 450 Gaussmeter was used to measure the applied magnetic field based on the Hall Effect. 


\section{Chapter Seven}

\section{Results and Discussions}

In this chapter, the results for $\mathrm{Gd}_{5}\left(\mathrm{Si}_{\mathrm{x}} \mathrm{Ge}_{1-\mathrm{x}}\right)_{4}$ were organized according to the $\mathrm{x}$ values in the three regions discussed in Chapter four:

(1) Region I: $0 \leq x \leq 0.3$

(2) Region II: $0.4 \leq x \leq 0.503$

(3) Region III: $0.575 \leq x \leq 1$

In each region a composition was selected to study the behavior in the vicinity of transformation temperature by thermal expansion measurements and magnetostriction measurements. 


\section{1 $\mathrm{Gd}_{5}\left(\mathrm{Si}_{\mathrm{x}} \mathrm{Ge}_{4-\mathrm{x}}\right)$ with $\mathrm{x}$ range of $0<x \leq 0.3$}

\subsubsection{Experimental details}

In this region, we selected a polycrystal sample $\mathrm{Gd}_{5}\left(\mathrm{Si}_{0.15} \mathrm{Ge}_{3.85}\right)\left(\left(\mathrm{Gd}_{5}\left(\mathrm{Si}_{\mathrm{x}} \mathrm{Ge}_{1-\mathrm{x}}\right)_{4}\right.\right.$ with $\mathrm{x}=$ 0.0375), which was prepared by arc-melting a stoichiometric mixture of pure components in an argon atmosphere under normal pressure. The sample used the same purity $\mathrm{Si}$ and $\mathrm{Ge}$ starting materials (both $>99.99$ at.\%). Ames Laboratory (AL) Gd $(99.8 \%$ pure, the major impurities (in atomic ppm) of which were: 440-O, 200-C, 160-H) was used for Gd content, the major impurities (in atomic ppm) of AL Gd were: 440-O, 200-C, 160-H. Mass losses after arc-melting were less than $0.5 \mathrm{wt} \%$, so the alloy chemical compositions were assumed to be to a first approximation unchanged in the final product. The thermal expansion measurements were conducted using the quarter bridge strain-gauge method in a Janis Research 2-stage closed cycle helium refrigeration. The sample was cooled down from room temperature down to the $15 \mathrm{~K}$. At $15 \mathrm{~K}$, a magnetic field of 2 Tesla was then applied to trigger the transition. Then, after the magnetic field was removed, magnetostriction measurements were carried out within $\pm 10 \mathrm{kOe}, \pm 20 \mathrm{kOe}$. When magnetostriction measurements were finished, the sample was heated back to room temperature with zero magnetic fields.

\subsubsection{Results and discussions}

As pointed out in previous chapter, in the region of $0 \leq x \leq 0.3$, when the sample is cooled down from the room temperature, there are two kinds of transitions that occur. One is the second order transition at higher temperature; it is from paramagnetic state to antiferromagnetic state with the same crystal structure: orthorhombic structure with the 
$\mathrm{Si}(\mathrm{Ge})-\mathrm{Si}(\mathrm{Ge})$ interlayer bonds broken [17], as shown in Fig. 4-2. It is interesting to notice that in the thermal expansion measurement shown in Fig.7-1, there is no abnormaly observed when the sample is cooled down from room temperature down to $15 \mathrm{~K}$. From this, it can be inferred that this kind magnetic transition makes no contribution to thermal expansion. This result is consistent with the finding in ref [45 ] by x-ray method. However, when a magnetic field of $20 \mathrm{kOe}$ was applied, the strain value shows an abrupt jump from $-3022 \mathrm{ppm}$ to $767 \mathrm{ppm}$. This is believed to be due to the unusual properties of $\mathrm{Gd}_{5}\left(\mathrm{Si}_{\mathrm{x}} \mathrm{Ge}_{1-\mathrm{x}}\right)_{4}$ that its transition temperature can be shifted by magnetic field: AFM state to FM state, and $\mathrm{Sm}_{5} \mathrm{Ge}_{4}$ type orthorhombic to $\mathrm{Gd}_{5} \mathrm{Si}_{4}$ type orthorhombic [17]. The difference between this two types structures is that the inter-layer bonds in the $\mathrm{Sm}_{5} \mathrm{Ge}_{4}$ type are all broken, while in $\mathrm{Gd}_{5} \mathrm{Si}_{4}$ type they are all connected. According to ref [34], a magnetic field can trigger this transition, but partially reversible between $10 \mathrm{~K}$ and $20 \mathrm{~K}$, therefore, our result in Fig.7-1 can be explained that the transition temperature was raised across $15 \mathrm{~K}$, and a structure change occurs which lead to a discontinuous change in strain. 


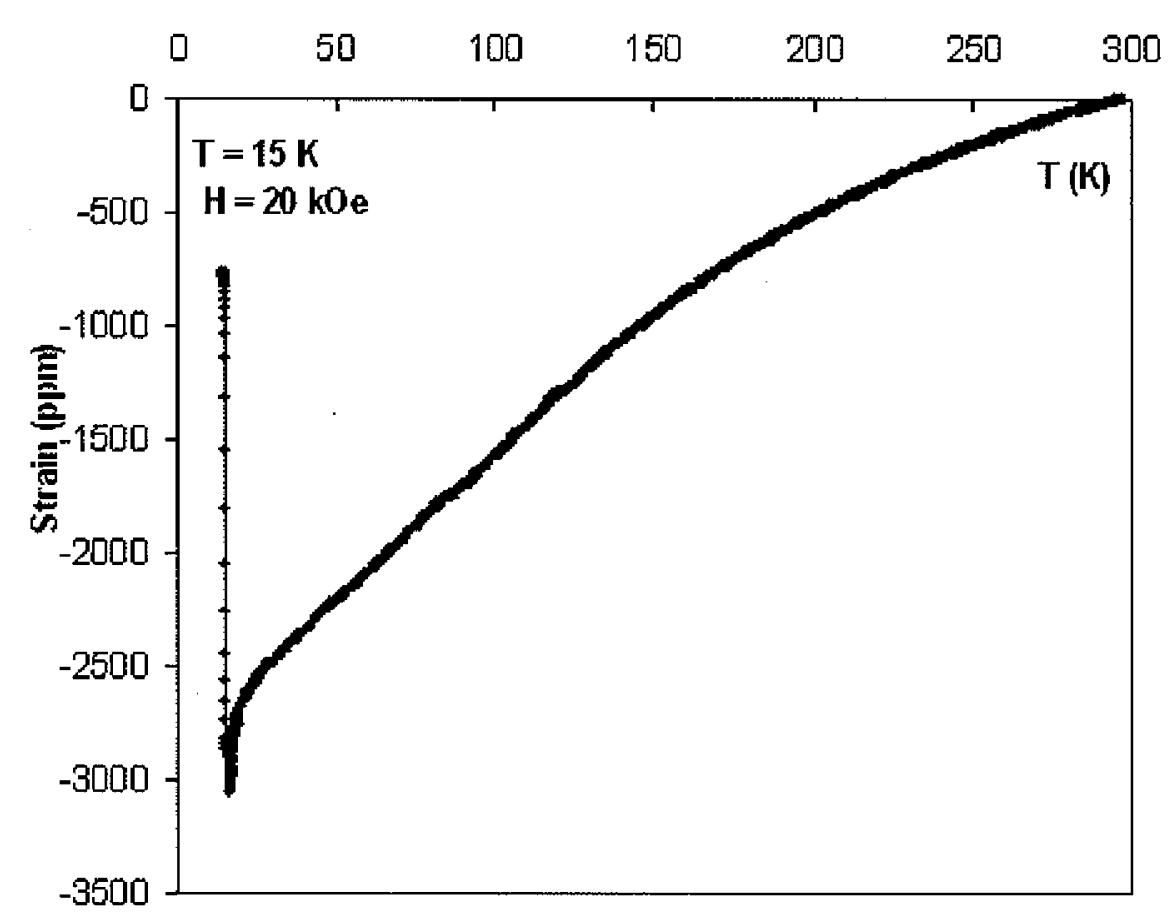

Fig.7-1 Thermal expansion measurement for polycrystalline sample $\mathrm{Gd}_{5}\left(\mathrm{Si}_{0.15} \mathrm{Ge}_{3.85}\right)$ on cooling, at $\mathrm{T}=15 \mathrm{~K}$, a magnetic field of 2 Tesla was applied.

After the magnetic field was removed, the strain returned to $2100 \mathrm{ppm}$ along the same path without showing a hysteresis, as shown below Fig.7-2. This result is similar to the results found in ref [35], where it is pointed out: for Ge-rich region, such as a polycrystal sample of $\mathrm{Gd}_{5}\left(\mathrm{Ge}_{4}\right)$, when it is cooled down to $5 \mathrm{~K}$ with zero field, there is no so-called first order AFM $\rightarrow$ FM transition found. However, when a magnetic field is applied, a nonreversible transition from $A F M \rightarrow F M$ happens, and it remains ferromagnetic state when the applied magnetic field is removed. When the sample is heated back to room temperature without magnetic field, a first order martensitic-like magnetocrystalline transition from FM $\leftrightarrow$ AFM happens at $\mathrm{T}=25 \mathrm{~K}$ as shown in the Fig.7-2. Similar behavior has been reported for a polycrystalline sample of $\mathrm{Gd}_{5} \mathrm{Ge}_{4}$ in ref $[36,37]$ via electrical resistivity studies. The transition temperature 
between AFM $\leftrightarrow$ FM found here is different from the phase diagram published in ref [14], indicates the necessary changes of phase diagram. In addition, the Neel temperature $T_{N}$ between $\mathrm{AFM} \leftrightarrow \mathrm{PM}$ has not been found by the thermal expansion measurements, other measurements techniques, such as specific heat capacity measurement, or magnetoresistance measurement could be used to find it.

When the applied magnetic field was removed, the sample retained its partially ferromagnetic state at $T=15 \mathrm{~K}$. Magnetostriction measurements were conducted for $|\mathrm{H}|_{\max }$ $=10 \mathrm{kOe}$ and $20 \mathrm{kOe}$, see Fig.7-3, and Fig.7-4 respectively. When the magnetic field was cycled within $\pm 10 \mathrm{kOe}$, the magnetostriction result showed a small magnitude value, the maximum strain was about $350 \mathrm{ppm}$. This result indicates that the sample is actually in ferromagnetic state throughout the range of fields measured. 


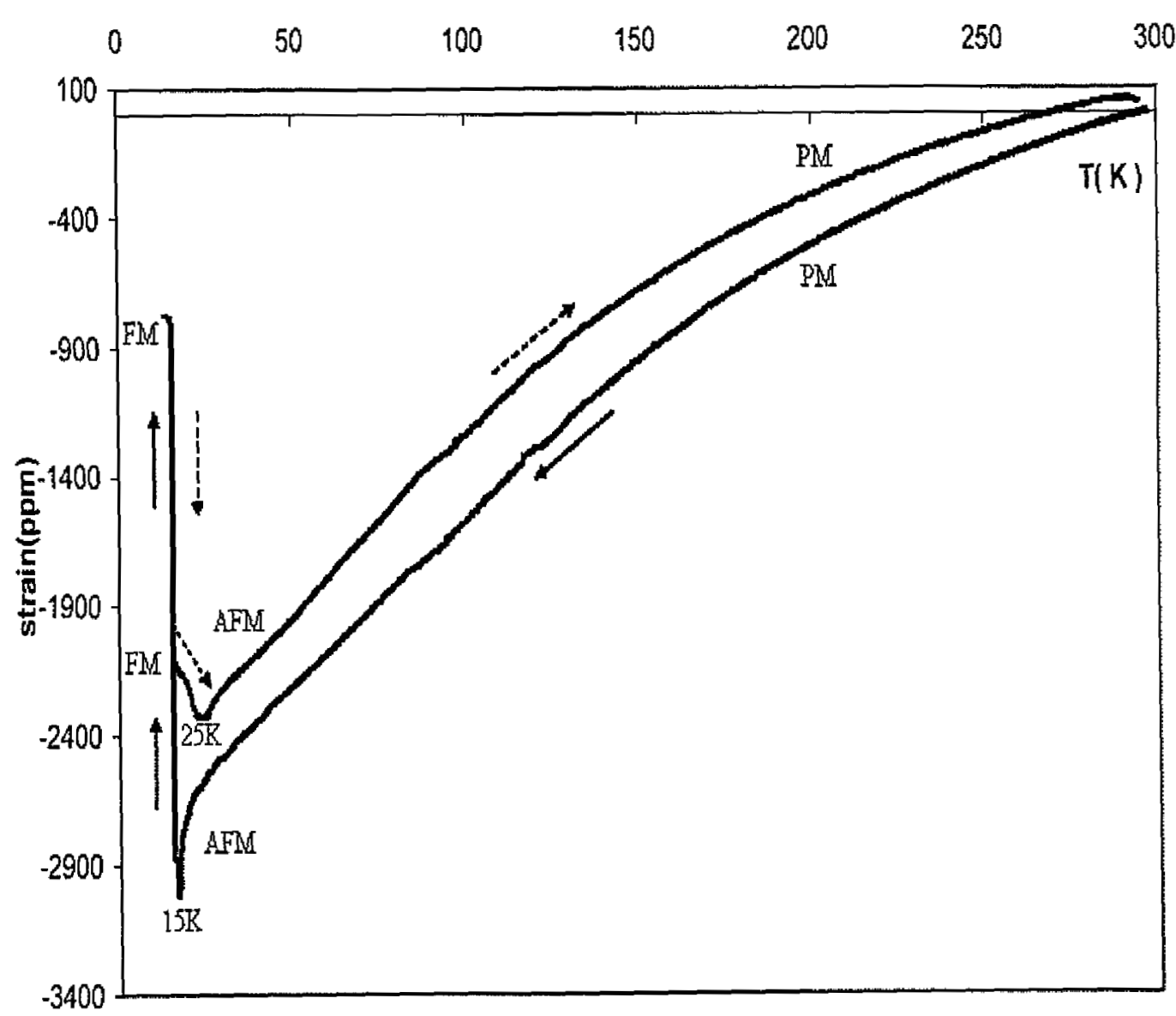

Fig.7-2 Thermal expansion measurement for polycrystal $\mathrm{Gd}_{5}\left(\mathrm{Si}_{0.15} \mathrm{Ge}_{3.85}\right)$ sample. A magnetic field of $20 \mathrm{kOe}$ was applied at $\mathrm{T}=15 \mathrm{~K}$, then removed at $\mathrm{T}=15 \mathrm{~K}$. Heating curve from $15 \mathrm{~K}$ to $T=296 \mathrm{~K}$ was obtained under zero magnetic field. At $\mathrm{T}=25 \mathrm{~K}$, first order $\mathrm{FM}$ to AFM magnetocrystalline transition happens. 


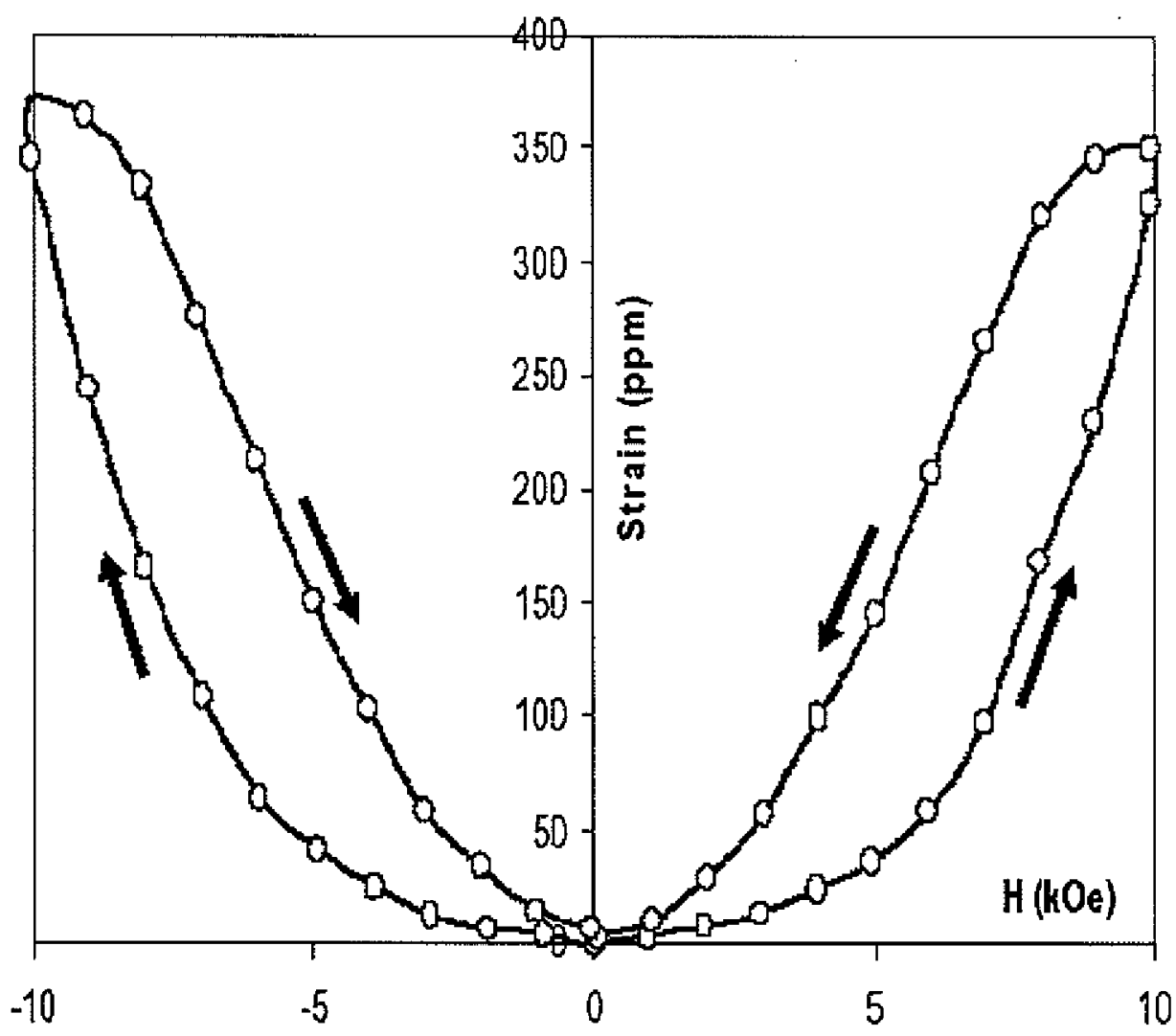

Fig.7-3 Magnetostriction at $\mathrm{T}=15 \mathrm{~K}$ for polycrystalline sample $\mathrm{Gd}_{5}\left(\mathrm{Si}_{0.15} \mathrm{Ge}_{3.85}\right)$ with $\mid \mathrm{H}_{\max }$ $\mid=10 \mathrm{kOe}$

When magnetostriction measurement was carried out at $\mathrm{T}=15 \mathrm{~K}$ under $\left|\mathrm{H}_{\max }\right|=20 \mathrm{kOe}$, as shown below, the result shows a giant magnetostriction, maximum magnetostriction value is about $1350 \mathrm{ppm}$, it comes to be saturated at $\mathrm{H}= \pm 20 \mathrm{kOe}$. 


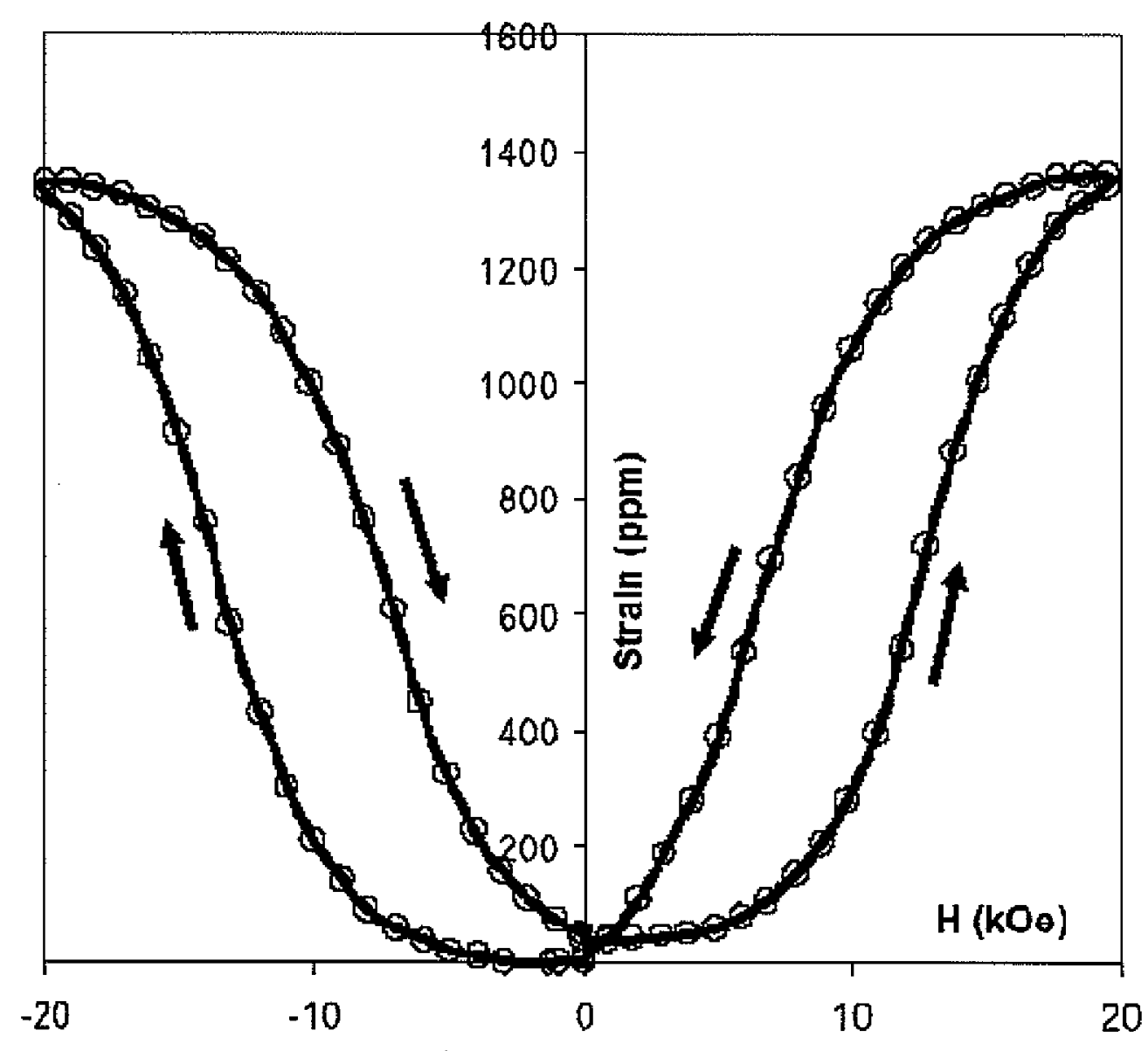

Fig.7-4. Giant Magnetostriction at $\mathrm{T}=15 \mathrm{~K}$ for polycrystalline sample $\mathrm{Gd}_{5}\left(\mathrm{Si}_{0.15} \mathrm{Ge}_{3.85}\right)$ with $\left|\mathrm{H}_{\max }\right|=20 \mathrm{kOe}$

After magnetostriction measurement, the sample was heated back to room temperature under zero magnetic fields, see the heating curve as shown in the Fig.7-2.

\section{Conclusions}

In the Ge-rich region of $\mathrm{Gd}_{5}\left(\mathrm{Si}_{x} \mathrm{Ge}_{4-\times}\right)$, such as $\mathrm{Gd}_{5}\left(\mathrm{Si}_{0.15} \mathrm{Ge}_{3.85}\right)$ discussed above, there are several different behaviors compared to the other regions that have been revealed using the magnetostriction and thermal expansion measurement in this work. When the sample is cooled down to $15 \mathrm{~K}$, thermal expansion measurements did not reveal a first order transition 
at low temperature, and did not reveal a second order transition from PM state to AFM state at high temperature. However, an external magnetic field can induce an irreversible first order transition from $\mathrm{AFM}$ to $\mathrm{FM}$ at $\mathrm{T}=15 \mathrm{~K}$ which showed a discontinuous strain change. When the magnetic field was removed, the sample remained in its partially ferromagnetic state, this is confirmed by the magnetostriction measurements with $\left|\mathrm{H}_{\max }\right|=10 \mathrm{kOe}, 20 \mathrm{kOe}$ at $15 \mathrm{~K}$. Giant magnetostriction measurement of about $1350 \mathrm{ppm}$ was found when $\left|\mathrm{H}_{\max }\right|=$ $20 \mathrm{kOe}$. After magnetostriction measurement, it was also found that when the sample was heated back to room temperature under zero field, there was a first order transition FM $\leftrightarrow$ AFM which occurred at $\mathrm{T}=25 \mathrm{~K}$. 


\section{$7.2 \mathrm{Gd}_{5}\left(\mathrm{Si}_{x} \mathrm{Ge}_{1-\mathrm{x}}\right)_{4}$ with $\mathrm{x}$ range of $0.4<x \leq 0.503$}

In this region, we selected a single crystal sample $\mathrm{Gd}_{5}\left(\mathrm{Si}_{1.95} \mathrm{Ge}_{2.05}\right)(\mathrm{x}=0.4875)$, three polycrystal samples $\mathrm{Gd}_{5}\left(\mathrm{Si}_{2.09} \mathrm{Ge}_{1.91}\right)(\mathrm{x}=0.5225)$, two of them were prepared by arcmelting method but with different purity grade Gd metals, one was prepared by induction melting with commercial grade $\mathrm{Gd}$, and a single crystal cubic sample $\mathrm{Gd}_{5}\left(\mathrm{Si}_{2} \mathrm{Ge}_{2}\right)(\mathrm{x}=0.5)$, Thermal expansion measurements, magnetostriction measurements, and VSM measurements were made on the same single crystal sample along different axes.

\subsubsection{Experimental Details}

The $\mathrm{Gd}_{5}\left(\mathrm{Si}_{1.95} \mathrm{Ge}_{2.05}\right)$ single crystal $(5 \mathrm{~mm} \times 4 \mathrm{~mm} \times 1 \mathrm{~mm})$, and $\mathrm{Gd}_{5}\left(\mathrm{Si}_{2} \mathrm{Ge}_{2}\right)(4 \mathrm{~mm} \times 4 \mathrm{~mm} \times$ $4 \mathrm{~mm}$ ) were grown by the Bridgman method. Appropriate quantities of gadolinium $99.996 \%$ (metals basis, wt. \%), silicon (99.9999\%) and germanium (99.999\%) were cleaned and arc melted several times under an argon atmosphere. The as-cast ingot was electron beam welded into a tungsten Bridgman style crucible for crystal growth. The ingot was then heated to $2000^{\circ} \mathrm{C}$ and held at this temperature for 1 hour to allow thorough mixing before withdrawing the sample from the heat zone at a rate of $4 \mathrm{~mm} / \mathrm{hr}$. The as-grown crystal was oriented by back-reflection Laue X-ray diffraction and the crystallographic directions assigned using x-ray diffraction two-theta scans of the single crystal. The sample was cut by spark cutting and the faces were polished using standard metallographic techniques.

Two polycrystalline $\mathrm{Gd}_{5}\left(\mathrm{Si}_{2.09} \mathrm{Ge}_{1.91}\right)$ samples were prepared by arc-melting a stoichiometric mixture of pure components in an argon atmosphere under normal pressure. The two samples used the same purity Si and Ge starting materials (both >99.99 at.\%). One of the samples was 
prepared using Ames Laboratory (AL) Gd ( $99.8 \%$ pure), the major impurities (in atomic ppm) of which were: $440-\mathrm{O}, 200-\mathrm{C}, 160-\mathrm{H}$. Sample 2 was fabricated by using commercial purity $\mathrm{Gd}(96.9$ at. $\%$ pure) which contained $18300-\mathrm{O}, 4300-\mathrm{C}, 4300-\mathrm{N}$ and $3700-\mathrm{F}$ (in atomic ppm). Mass losses after arc-melting were less than $0.5 \mathrm{wt} \%$, so the alloy chemical compositions were assumed to be to a first approximation unchanged in the final product.

The polycrystalline $\mathrm{Gd}_{5}\left(\mathrm{Si}_{2.09} \mathrm{Ge}_{1.91}\right)$ sample, which was prepared by induction melting of a stoichiometric mixture of pure components in an argon atmosphere under normal pressure, has the same high purity $\mathrm{Si}$ and Ge starting materials as the above two polycrystal sample $\mathrm{Gd}_{5}\left(\mathrm{Si}_{2.09} \mathrm{Ge}_{1.91}\right)$. Thermal expansion measurements with different magnitudes of magnetic field were conducted over different temperature ranges as shown in the figures. Magnetostriction measurements were also carried out for different temperatures, see the results shown later. In-situ magnetic force microscopy (MFM) was used to observe the phase transformation. This was carried out by using an MFM equipped with a heating-cooling stage.

The thermal expansion measurements and magnetostriction measurement techniques were similar to those described in section 7.1. The location of the steepest point of the strain versus temperature curve was taken to be the transition temperature. However, there were some minor experimental differences, such as temperature range for the measurements, the applied magnetic fields, etc. Therefore, they are discussed separately here.

For single crystal $\mathrm{Gd}_{5}\left(\mathrm{Si}_{1.95} \mathrm{Ge}_{2.05}\right)$, the dc magnetic field was produced by a standard electromagnet with full range of \pm 2.5 Tesla. Thermal expansion measurements were carried out initially without applying a magnetic field. Then, a series of thermal expansion 
measurements were made for different constant applied magnetic field strengths along the $a$ axis due to the shape of sample. The magnetic field was maintained constant throughout the measurement cycle. The values of the field strength used were: $0,1.0,1.5,2.0,2.5$ Tesla.

For the single crystal sample $\mathrm{Gd}_{5}\left(\mathrm{Si}_{2} \mathrm{Ge}_{2}\right)$, measurements were made along $\mathrm{a}, \mathrm{b}$, c axis, Along the $c$ axis and thermal expansion measurement was made without a magnetic field applied, the sample was cooled from room temperature through the phase transition down to $15 \mathrm{~K}$, and then was heated up through the transition for each measuring cycle. With magnetic field applied, thermal expansion measurements were conducted along a, b and c axis, detailed temperature range and magnitude are shown on the graphs in each subsection later.

\subsubsection{Results and Discussions}

The results will be separated into three part based on their composition. Conclusions will be made on each part separately.

\section{I. $\mathrm{Gd}_{5}\left(\mathrm{Si}_{1.95} \mathrm{Ge}_{2.05}\right)$}

Fig.7-5 shows the linear thermal expansion measured along the $a$-axis in zero applied magnetic field. The strain $\lambda\left(\lambda=\Delta 1 / 1_{0}\right.$, where $l_{0}$ denotes the original length $)$ showed an abrupt change during the cooling and heating processes due to the phase transformation. The transformation is also manifested as a sharp peak in the plot of the differential thermal expansion against temperature as shown in Fig.7-6. On cooling, there are two transformations that happen simultaneously at $267 \mathrm{~K}$. One is the crystalline structural transition: monoclinic $\rightarrow$ orthorhombic. The other is the magnetic transition: paramagnetic

$\rightarrow$ ferromagnetic. An ordinary magnetic transition between paramagnetic state and 
ferromagnetic state is an order-disorder transition which is not accompanied by a simultaneous crystallographic transformation.

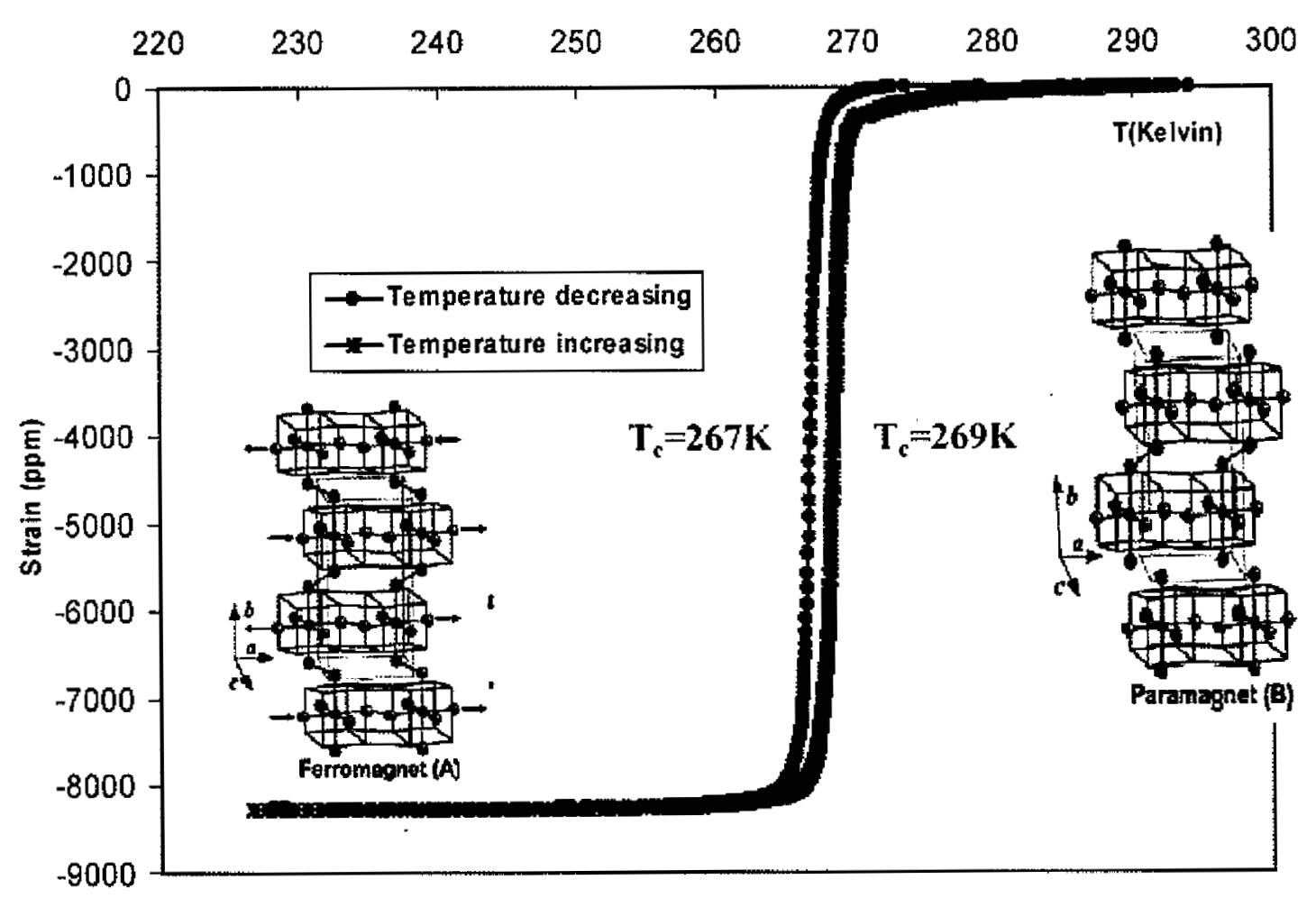

Fig.7-5. Thermal expansion of $\mathrm{Gd}_{5}\left(\mathrm{Si}_{1.95} \mathrm{Ge}_{2.05}\right)$ along the a-axis under zero field. $\mathrm{T}_{\mathrm{c}}$ is $267 \mathrm{~K}$ on cooling and $269 \mathrm{~K}$ on heating. The crystal pictures above are from ref [17].

In this material it was found that on heating the transition occurs at a higher temperature of $269 \mathrm{~K}$. The temperature hysteresis is therefore $2 \mathrm{~K}$. One of the features of a first-order phase transformation is the presence of hysteresis in the transition temperature which is due to the latent heat at the transition temperature for a first order transition. Therefore the thermal expansion data confirm that the phase transformation in this material is an unusual simultaneous first order magnetic-structural phase transformation; that is magnetically an order-disorder transition, but structurally an order-order transition. In these materials the structural or crystalline phase transition can be caused either by a change in temperature or a 
change in magnetic field. Therefore the structural transition can be induced by the presence of a magnetic field at temperatures above the normal transition temperature. For example,

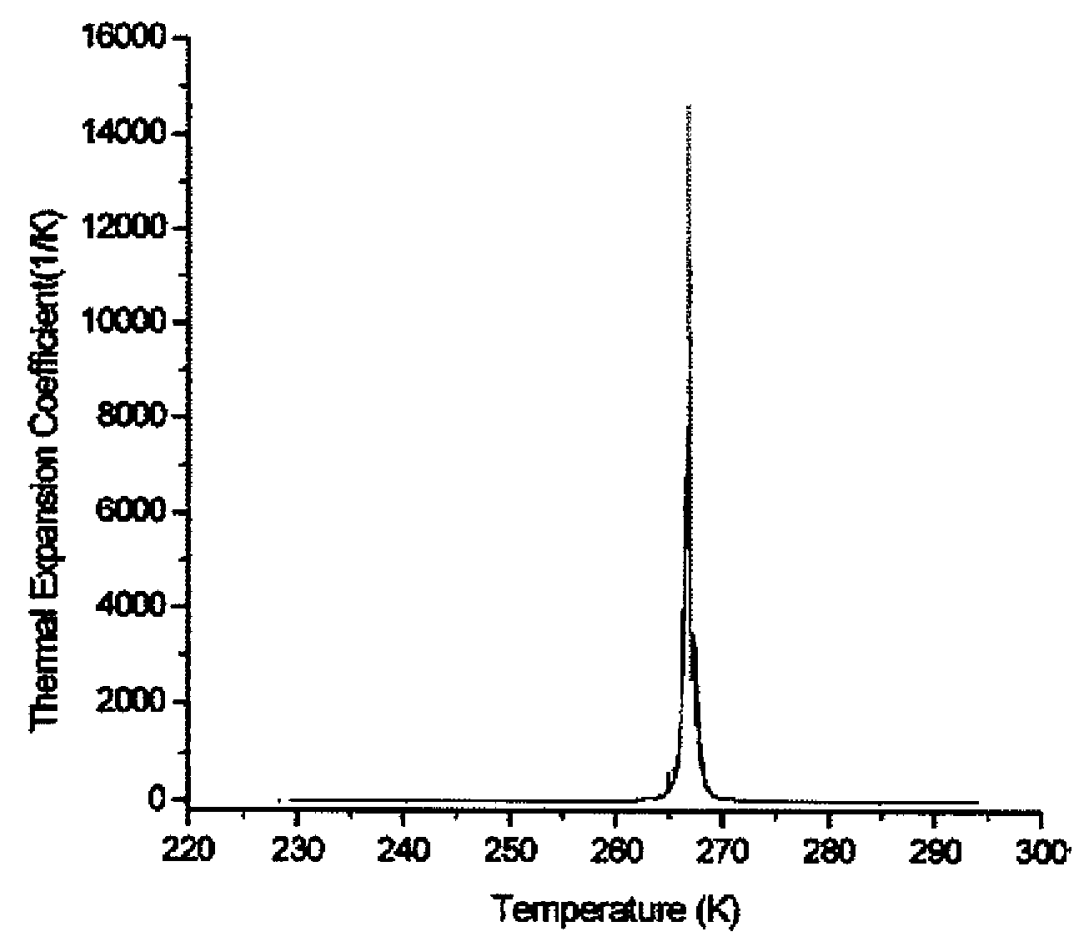

Fig.7-6 Dependence of the differential thermal expansion coefficient $\frac{d l}{d T} \cdot \frac{1}{l_{0}}$ on temperature while cooling down the sample.

in Fig. 7-7 (a) (b) (c), which shows that the thermal expansion of $\mathrm{Gd}_{5}\left(\mathrm{Si}_{1.95} \mathrm{Ge}_{2.05}\right)$ under an applied field of 1, 1.5 and 2 Tesla. The transition temperatures under a 2 Tesla applied field were found to be $276 \mathrm{~K}$ on cooling and $279 \mathrm{~K}$ on heating which are higher than the corresponding transition temperatures measured without any applied field. The dependence of the transition temperature of single crystal $\mathrm{Gd}_{5}\left(\mathrm{Si}_{1.95} \mathrm{Ge}_{2.05}\right)$ on the magnetic field along the $a$-axis is shown in Fig. 7-8. On either cooling or heating the transition temperature increases linearly with magnetic field at a rate of $4.9 \mathrm{~K} /$ Tesla. A plot of Tc against fields is shown in Fig. 7-8. 
The magnetic structure of $\mathrm{Gd}_{5}\left(\mathrm{Si}_{\mathrm{x}} \mathrm{Ge}_{1-\mathrm{x}}\right)_{4}$ is determined by two competing factors: the exchange interaction between Gd atoms which tends to order the magnetic moments, and the thermal energy which tends to disorder the magnetic moments. When the former is greater the material is ferromagnetic. At the transition temperature they are equal. Above the transition temperature the thermal energy is greater and the material is magnetically disordered. Since the magnetic field is applied along one direction, in this case the $a$-axis, we assume that the magnetic field suppresses the mechanical vibration of $\mathrm{Gd}$ atoms. Therefore in order to disrupt the magnetic order in the presence of a magnetic field, additional thermal 


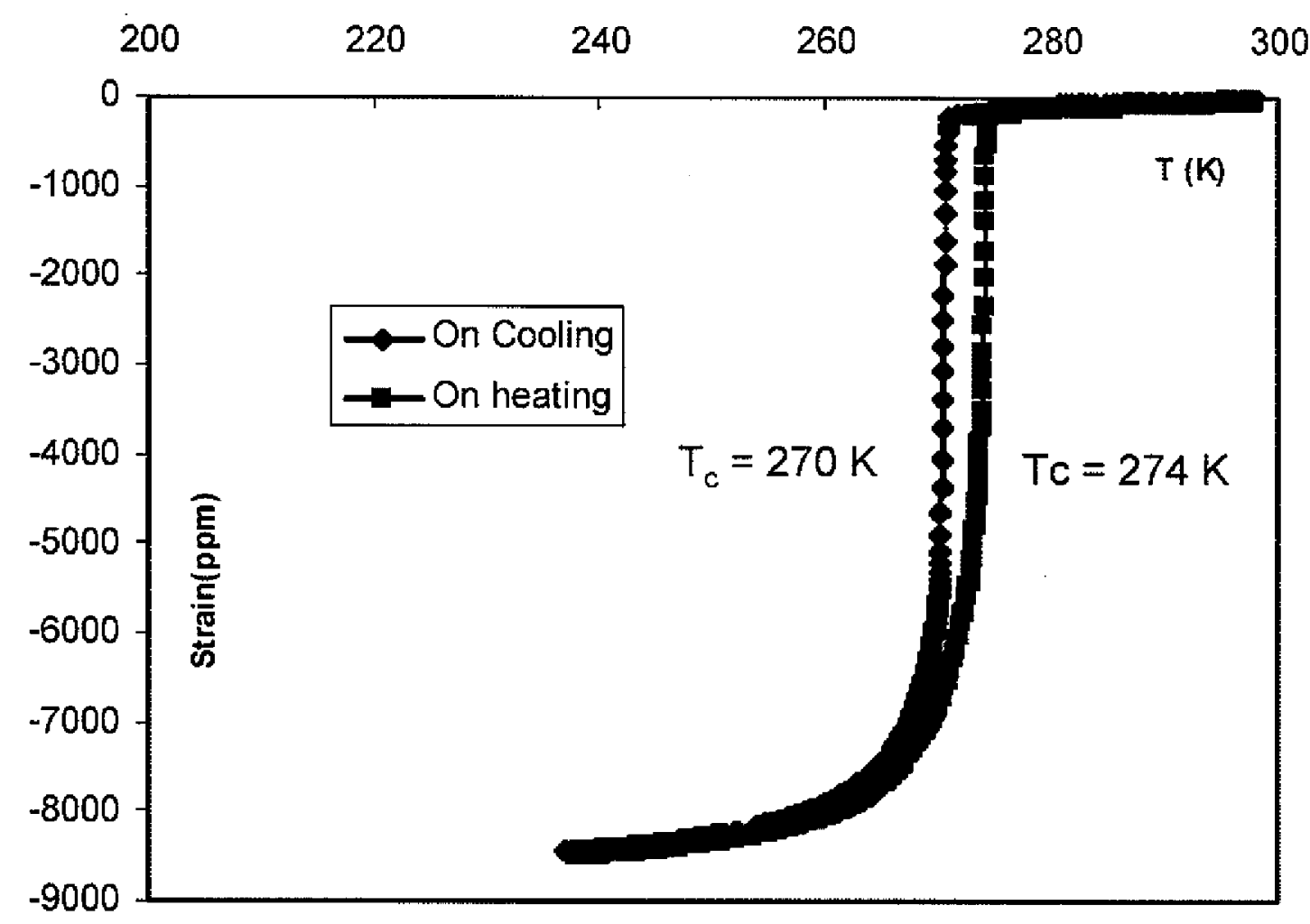

Fig 7-7 (a) . Thermal expansion of $\mathrm{Gd}_{5}\left(\mathrm{Si}_{1.95} \mathrm{Ge}_{2.05}\right)$ along the a-axis under an applied field of 1 Tesla. 


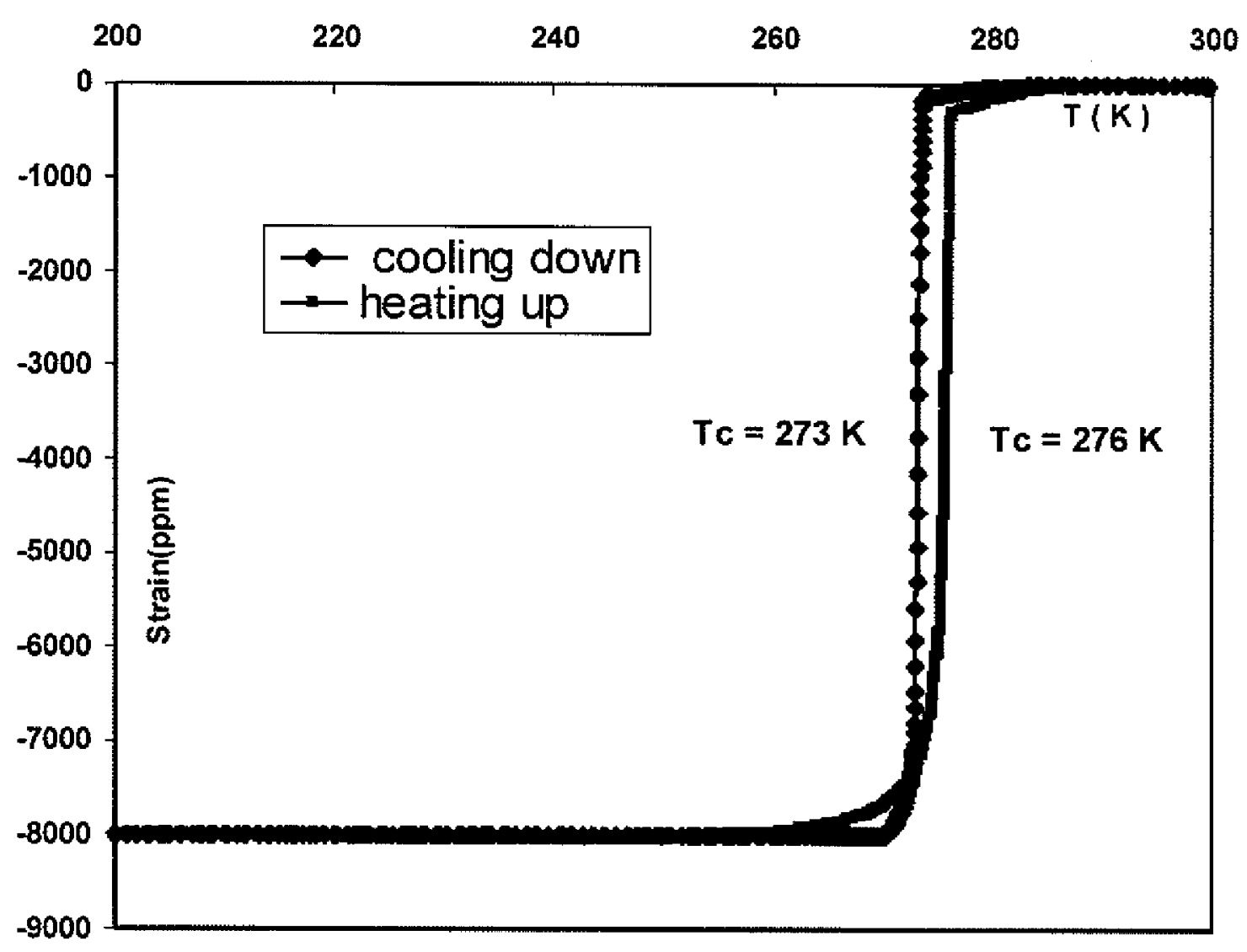

Fig 7-7 (b) . Thermal expansion of $\mathrm{Gd}_{5}\left(\mathrm{Si}_{1.95} \mathrm{Ge}_{2.05}\right)$ along the a-axis under an applied field of 1.5 Tesla. 


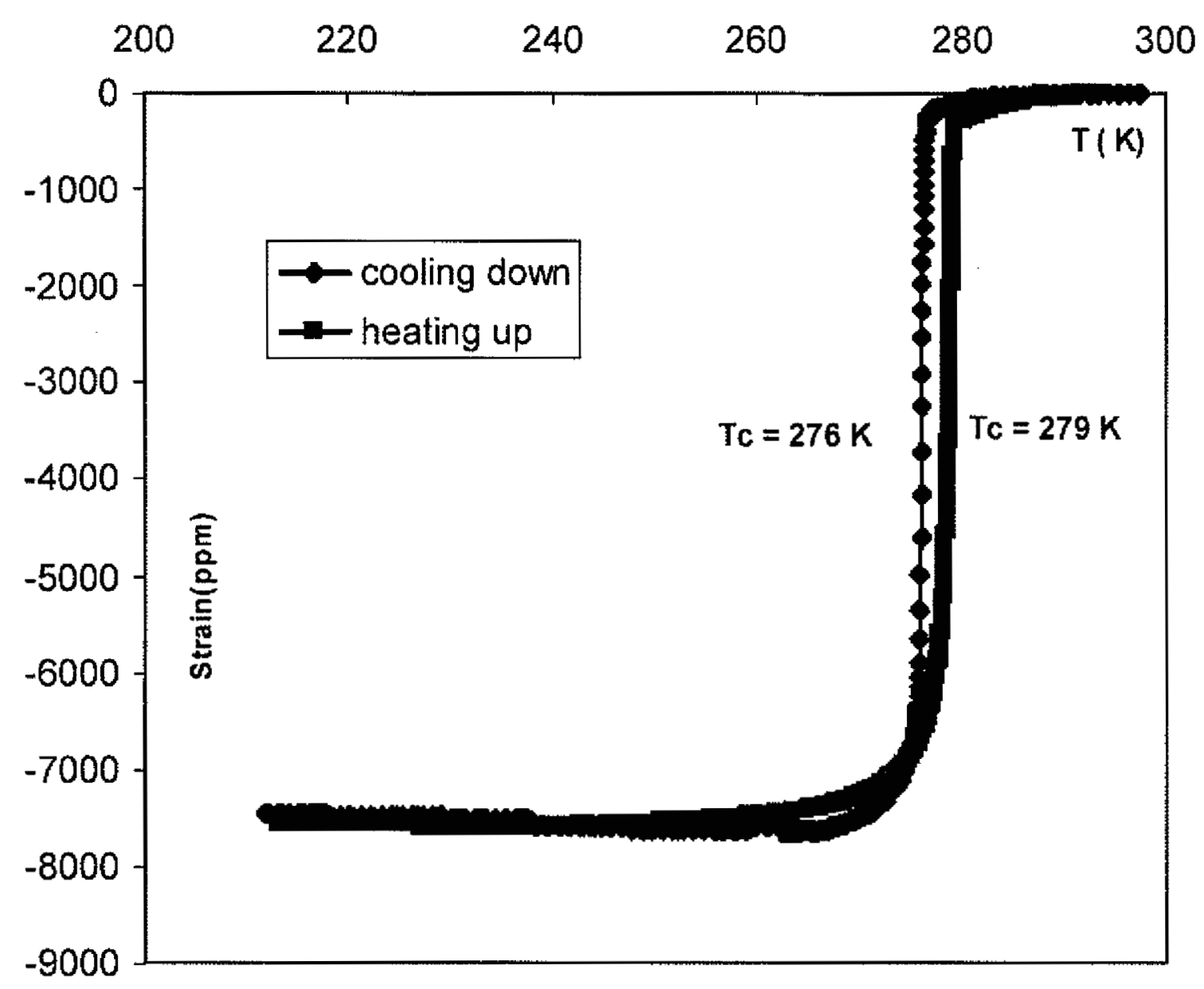

Fig 7-7 (c) Thermal expansion of $\mathrm{Gd}_{5}\left(\mathrm{Si}_{1.95} \mathrm{Ge}_{2.05}\right)$ along the a-axis under an applied field of 2.0 Tesla.

energy is required. The result is that the transition temperature is shifted toward a higher temperature when a magnetic field is applied. 


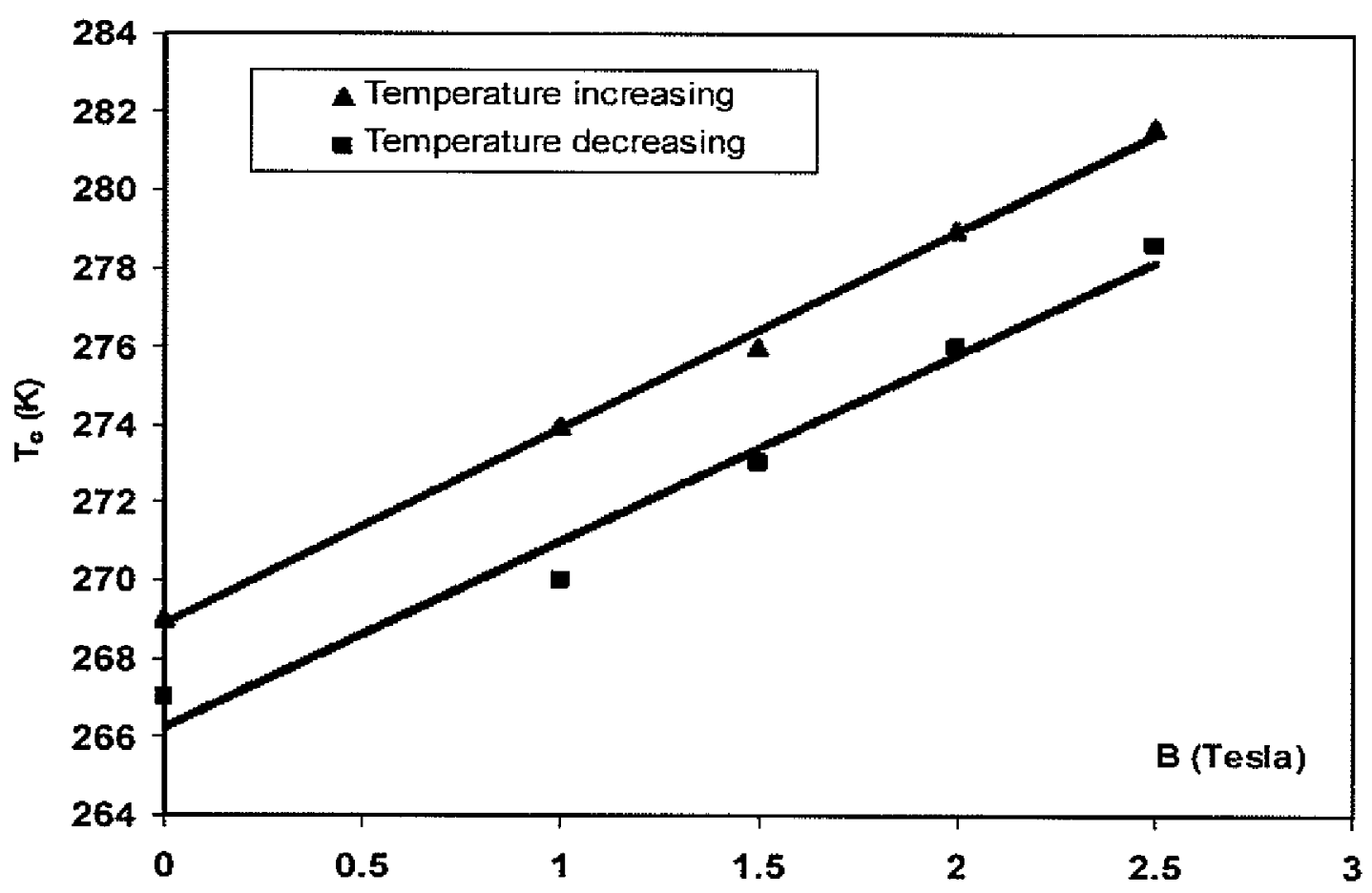

Fig 7-8 The dependence of transition temperature on the magnetic field applied along the aaxis.

The extra thermal energy $\Delta \mathrm{E}$ for each atom of $\mathrm{Gd}$ required to disorder the magnetic moments is equal to the magnetic energy $E_{H}$ exerted by the external magnetic field:

$$
\begin{aligned}
& \Delta E=\frac{n}{2} k_{B} \Delta T \\
& E_{H}=m \Delta B
\end{aligned}
$$

where $\mathrm{n}$ is the number of degrees of freedom, $\mathrm{k}_{\mathrm{B}}$ is the Boltzmann's constant $\left(1.38 \times 10^{-23}\right.$ $\left.\mathrm{J} \cdot \mathrm{K}^{-1}\right), \mathrm{m}$ is the magnetic moment of Gd atom $\left(7.9 \mu_{\mathrm{B}}=7.3 \times 10^{-23} \mathrm{~A} \cdot \mathrm{m}^{2}\right)$. Based on this assumption the expected rate of change of transition temperature with field is

$$
\frac{\Delta T}{\Delta B}=\frac{2 m}{n k_{B}} \approx \frac{10.6}{n} K \cdot T^{-1}
$$


The measured rate of change of transition temperature with field $(\Delta \mathrm{T} / \Delta \mathrm{B})_{\text {meas }}$ is $4.9 \mathrm{~K} / \mathrm{Tesla}$. So, in order to agree with the experimental data, the number of degrees of freedom appears to be

$$
n=\frac{10.6}{(\Delta T / \Delta B)_{\text {measured }}} \approx 2
$$

According to equation (3) $n=2$ would give the expected rate of change of transition temperature with field to be $5.3 \mathrm{~K} / \mathrm{Tesla}$, which is very close to the measured value of 4.9 K/Tesla. This suggests that the thermal energy required to overcome the effect of the applied field is equivalent to a system with only 2 degrees of freedom.

\section{Conclusions}

The linear thermal expansion along the $a$-axis of single crystal $\mathrm{Gd}_{5}\left(\mathrm{Si}_{1.95} \mathrm{Ge}_{2.05}\right)$ was measured for the first time. The results showed that the sudden change in strain is due to simultaneous magnetic and structural phase transformations. Both temperature and magnetic field influence the phase transformation. The sudden change in strain occurs at different temperatures depending on whether the temperature is being increased or decreased. This hysteresis in temperature is characteristic of a first order phase transformation. The temperature of the transition can also be increased by the presence of a magnetic field. An applied field shifts the transformation temperature by about $4.9 \mathrm{~K} / \mathrm{Tesla}$. It appears therefore that the magnetic field suppresses the vibration of the Gd atoms so that the magnetic and structural phase transitions are moved to higher temperature. 


\section{II. $\mathrm{Gd}_{5}\left(\mathrm{Si}_{2} \mathrm{Ge}_{2}\right)$}

\section{(a)Magnetization measurement along a, b, c axis:}

Magnetization along $\mathrm{a}, \mathrm{b}$, and $\mathrm{c}$ axis at $\mathrm{T}=15 \mathrm{~K}$ and $265 \mathrm{~K}$ are shown in Figs.7- 9 and 7-10. As shown in Fig.7-9, the $\mathrm{M}-\mathrm{H}$ curves for three axis were typical curves for a magnetic material, denoting the sample was in a ferromagnetic state at $\mathrm{T}=15 \mathrm{~K}$. A field of $10 \mathrm{kOe}$ can saturate each axis, but the $b$ axis needs less field for saturation, therefore, it is the easy axis, and $a$ axis is the hard axis, this results agrees with the finding in ref [39], where the VSM measurement was conducted at $265 \mathrm{~K}$. Here, the isothermal magnetization along the $\mathrm{b}$ axis was discussed in detail, as seen in Figs. 7-11 and 7-12.

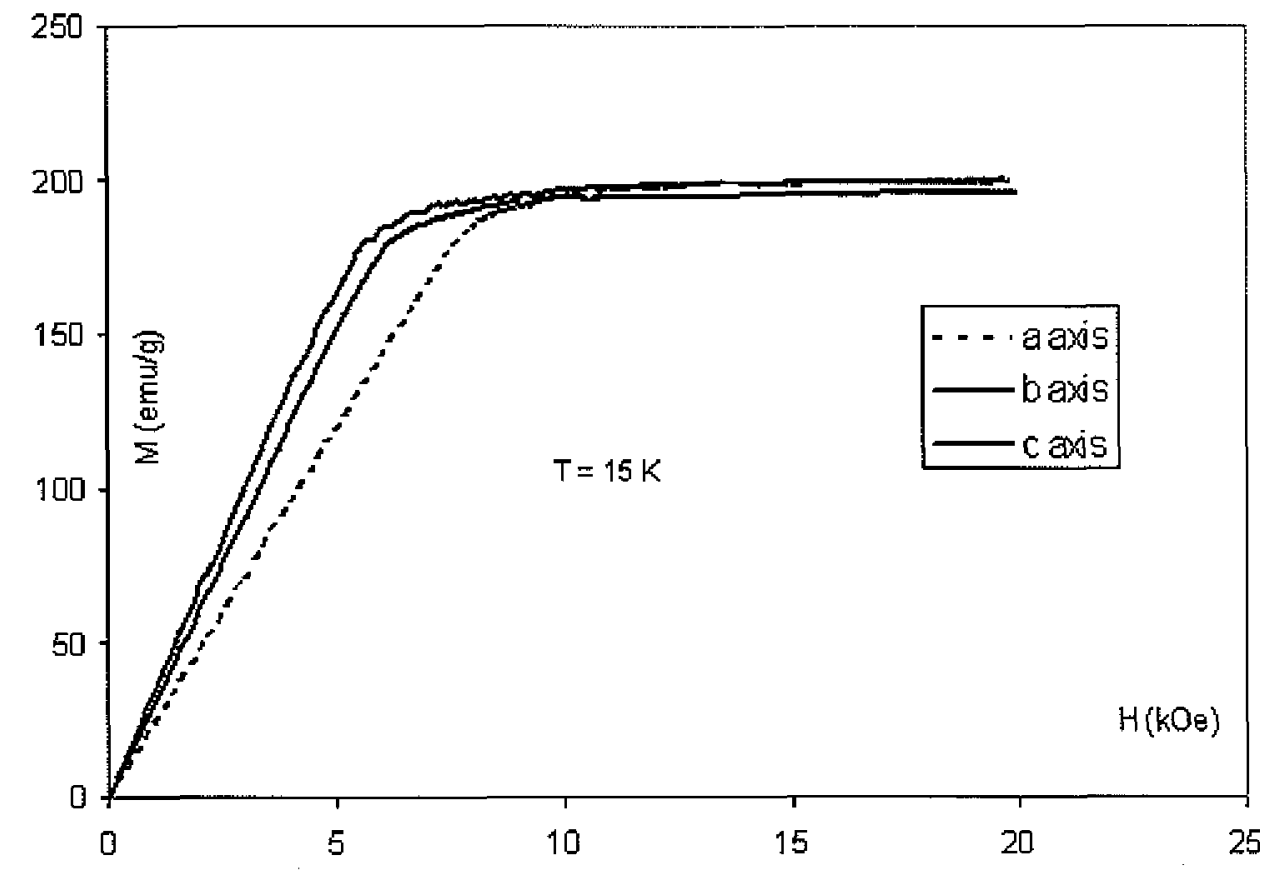

Fig. $7-9 \mathrm{M}$ vs. $\mathrm{H}$ along $\mathrm{a}, \mathrm{b}, \mathrm{c}$ axis at $\mathrm{T}=15 \mathrm{~K}$ 


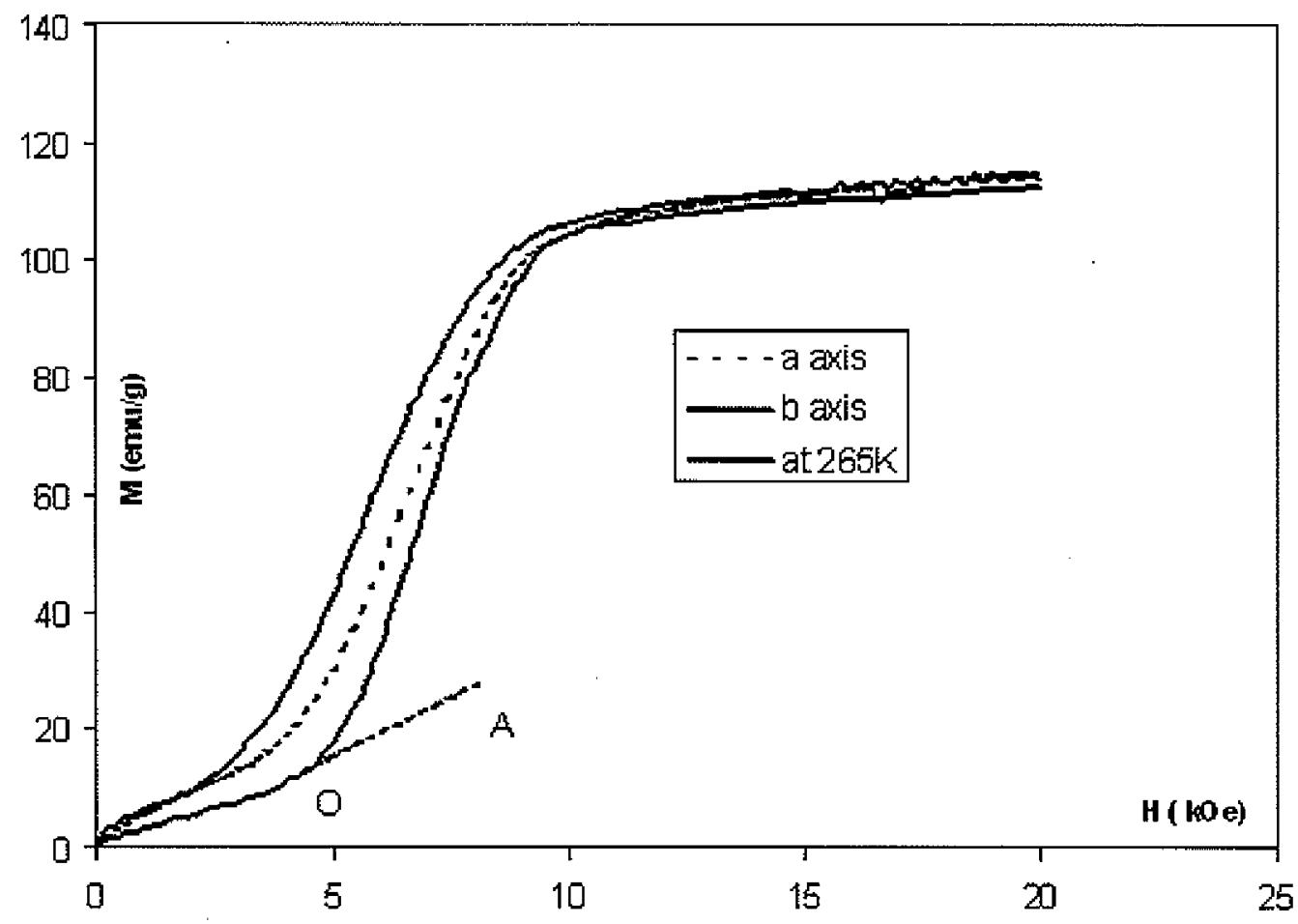

Fig. $7-10 \mathrm{M}$ vs. $\mathrm{H}$ along $\mathrm{a}, \mathrm{b}, \mathrm{c}$ axis at $\mathrm{T}=265 \mathrm{~K}$

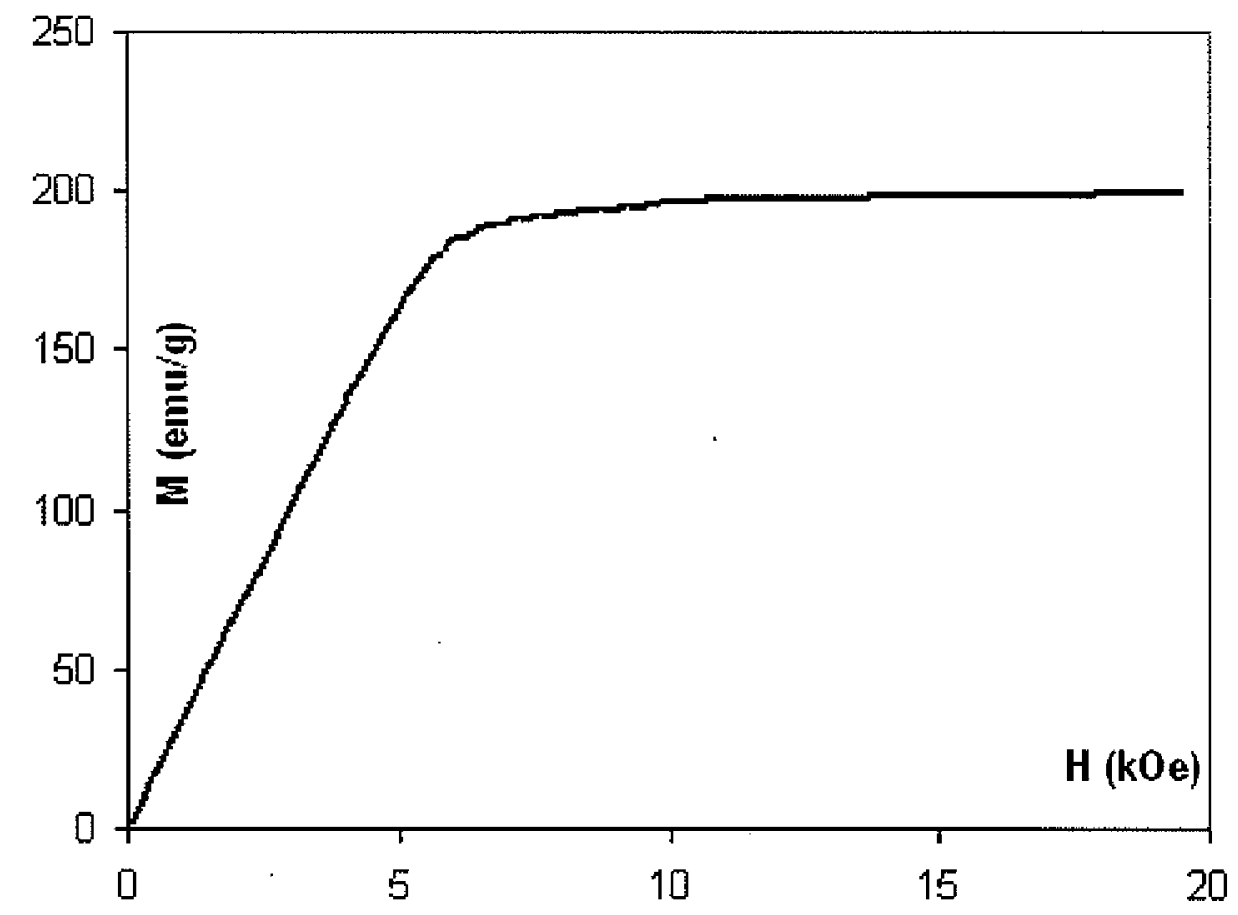


Fig.7- 11 Magnetization vs. $\mathrm{H}$ measured along $\mathrm{b}$ axis at $\mathrm{T}=15 \mathrm{~K}$ by using the SQUID

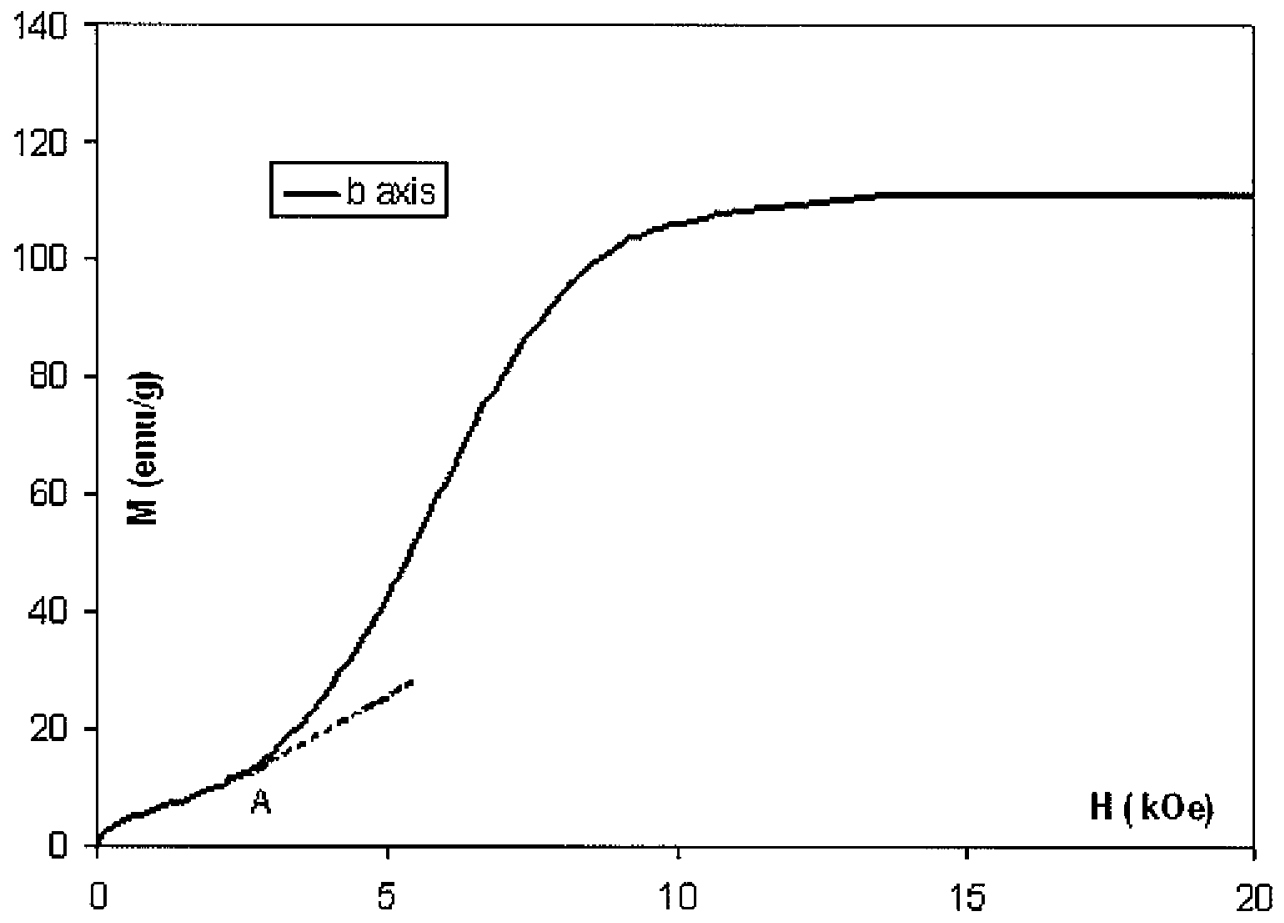

Fig.7-12 Magnetization VS H measured along $\mathrm{b}$ axis at $\mathrm{T}=265 \mathrm{~K}$ by using the SQUID

At $\mathrm{T}=265 \mathrm{~K}$, it is near the transition temperature ( $\mathrm{Tc}=259.5 \mathrm{~K}$ ) found under zero field, and at $\mathrm{T}=15 \mathrm{~K}$, it is far away from the transition temperature. At $\mathrm{T}=15 \mathrm{~K}$, as shown in Fig.7-11, the magnetization curve is a typical one for ferromagnetic materials. However, at $\mathrm{T}=265 \mathrm{~K}$, we observed a different shape curve, see Fig.7-12. Below the "A" point, the magnetization process was due to domain wall movement of a partial volume of the ferromagnetic material. Above A point, the magnetic field if strong enough will induce a transition from PM to FM, and results in a significant increase in magnetization for the remaining volume of PM. In other words, the PM (monoclinic) phase coexists with the FM (orthorhombic) phase. This is 
another characteristic of a first order transition: coexistence. When all the PM phase had transformed into FM phase, the magnetization gradually reached the saturation level.

At $15 \mathrm{~K}$, the saturation magnetization is about $200 \mathrm{emu} / \mathrm{g}\left(1.48 \times 10^{6} \mathrm{~A} / \mathrm{m}\right)$. However at 265 $\mathrm{K}$, it is about $100 \mathrm{emu} / \mathrm{g}\left(0.74 \times 10^{6} \mathrm{~A} / \mathrm{m}\right)$. A possible reason for it is that at low temperature, the net magnetic magnetization in each domain more easily rotates into the magnetic field direction, and therefore the magnetic moments become more aligned along the magnetic field direction.

\section{(b)Thermal expansion along a axis}

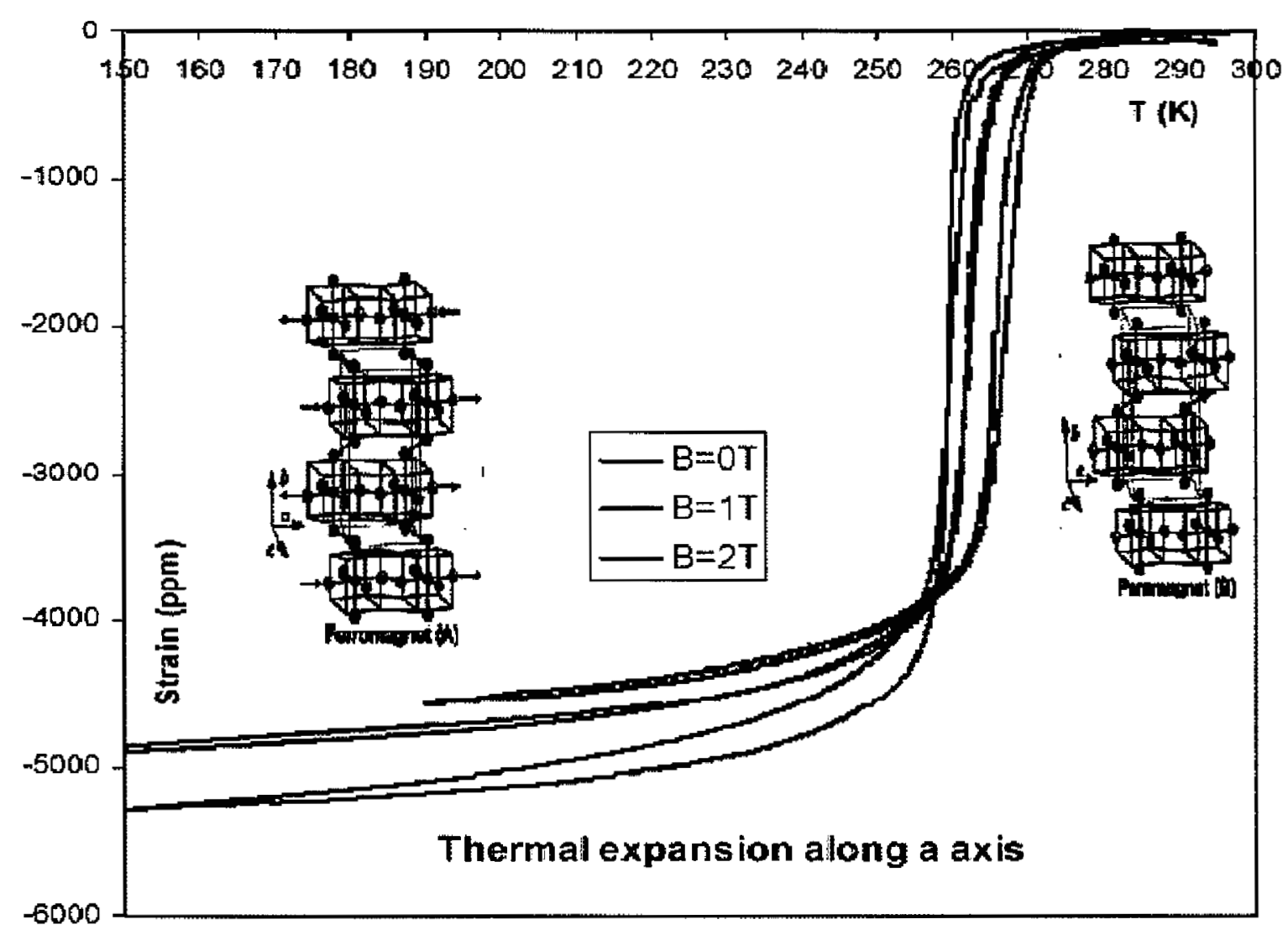

Fig.7- 13 Thermal expansion along a axis for $\mathrm{Gd}_{5}\left(\mathrm{Si}_{0.5} \mathrm{Ge}_{0.5}\right)_{4}$ showing the effect of applied magnetic field on the transition temperatures, the crystal structure pictures are from ref [17]. 
The thermal expansion measurement along the $a$ axis as shown in the Fig.7-13. At B $=0$ Tesla, the transition temperature is about $260 \mathrm{~K}$ on cooling, it is about $262 \mathrm{~K}$ on heating. The information about the coexistence found in the magnetization measurement discussed previously and the temperature hysteresis found here shows that it is a first order transition, where above the transition temperature, it is paramagnetic. Below the transition temperature, it is in a ferromagnetic state. The strain jump change at the transition temperature shows that there exists a structural transition. According to ref [14], it is due to the transition between the monoclinic structure (space group P112, $/$ a) and the orthorhombic structure (space group Pnma). For $\mathrm{Gd}_{5}\left(\mathrm{Si}_{\mathrm{x}} \mathrm{Ge}_{1-\mathrm{x}}\right)_{4}$ system, Choe [16] showed that the magnetic structural transitions are closely coupled with the crystal structural transitions by breaking or reforming the covalent $\mathrm{Si}(\mathrm{Ge})-\mathrm{Si}(\mathrm{Ge})$ bonds between the intra-layer building blocks by a shear movement of the building blocks along the a axis. The superexchange between $\mathrm{Gd}$ atoms within the adjacent layers is therefore believed to depend on these chemical bonds. It is well accepted that these two transformations happen simultaneously. Therefore, it can be inferred that any factors which can induce the breaking and reforming the $\mathrm{Si}(\mathrm{Ge})-\mathrm{Si}(\mathrm{Ge})$ bonds can trigger such as a unusual simultaneous transition. As shown above, temperature also can trigger this transition. Morellon, et al [37] have found the hydrostatic pressure can trigger this transition. As shown in Fig. 7-13, it was also found that the external magnetic field can trigger the transition for a single sample $\mathrm{Gd}_{5}\left(\mathrm{Si}_{2} \mathrm{Ge}_{2}\right)$ along its a axis, the results were shown in Fig. 714. 
As shown in Fig.7-13, the strain below Tc is about -5200 ppm, and deceasing with increasing magnetic field, and this indicates that the magnetostriction is possible with a positive value so that it can offset the thermal expansion due to the decreasing of temperature: This will be studied and discussed in detail later.

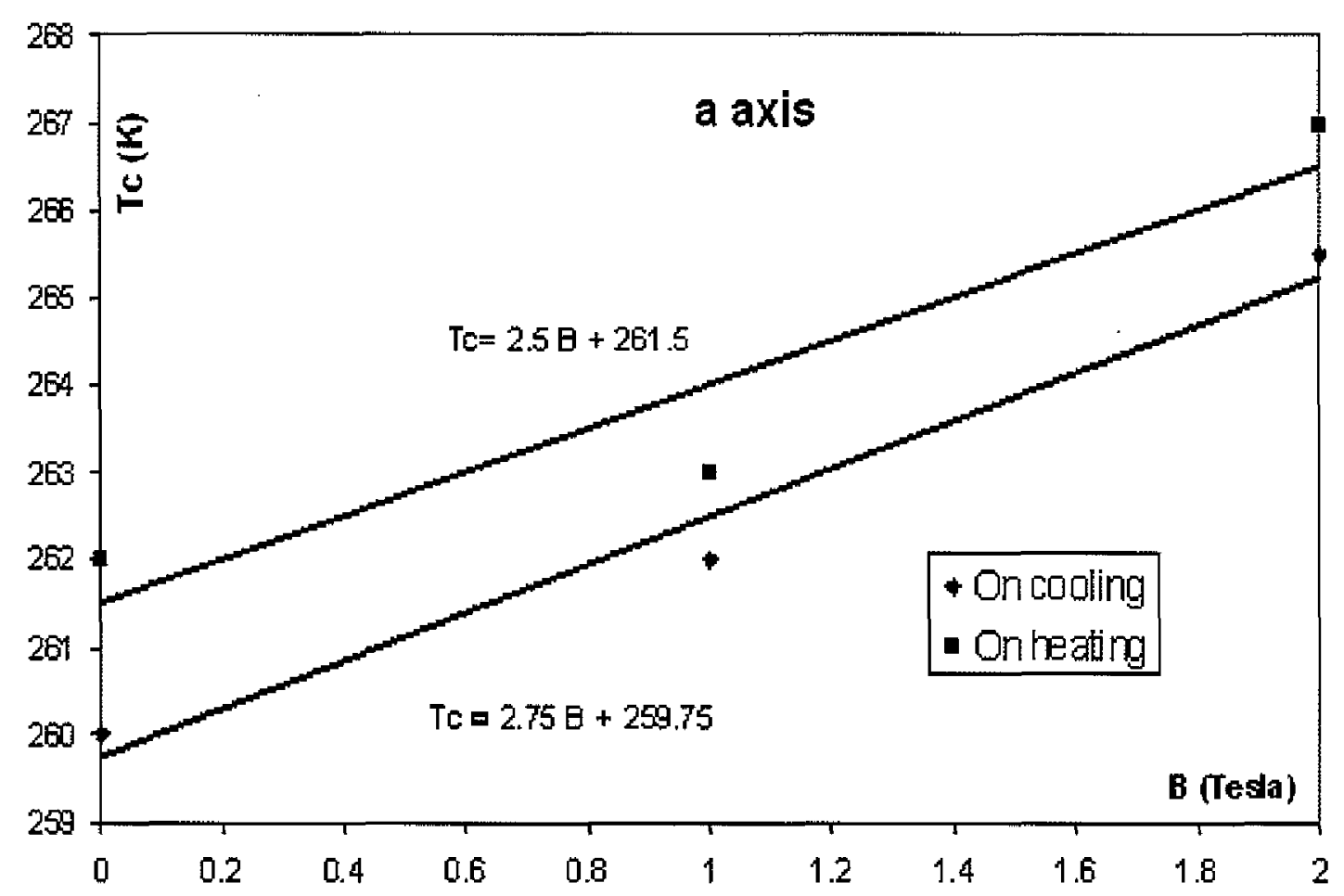

Fig.7-14 Tc dependence on magnetic field along a axis of $\mathrm{Gd}_{5}\left(\mathrm{Si}_{2} \mathrm{Ge}_{2}\right)$

\section{(c) Thermal expansion along the $b$ axis:}

Fig.7-15 shows the result of thermal expansion measurement under zero magnetic field along $\mathrm{b}$ axis. On cooling, Tc is found to be $259.5 \mathrm{~K}$, Tc on heating it was found to be $262.3 \mathrm{~K}$. The temperature hysteresis is about $3 \mathrm{~K}$, and this result also clearly shows that it is a first order transition. These transition temperatures found here are a little different from that found along the a axis. The abrupt change found in Fig.7-15 was also believed due to the 
combination of two transitions: a magnetic transition where spontaneous magnetization suddenly appears on cooling and disappears on heating, and the crystal structure transforms from monoclinic to orthorhombic on cooling, and from orthorhombic to monoclinic on heating.

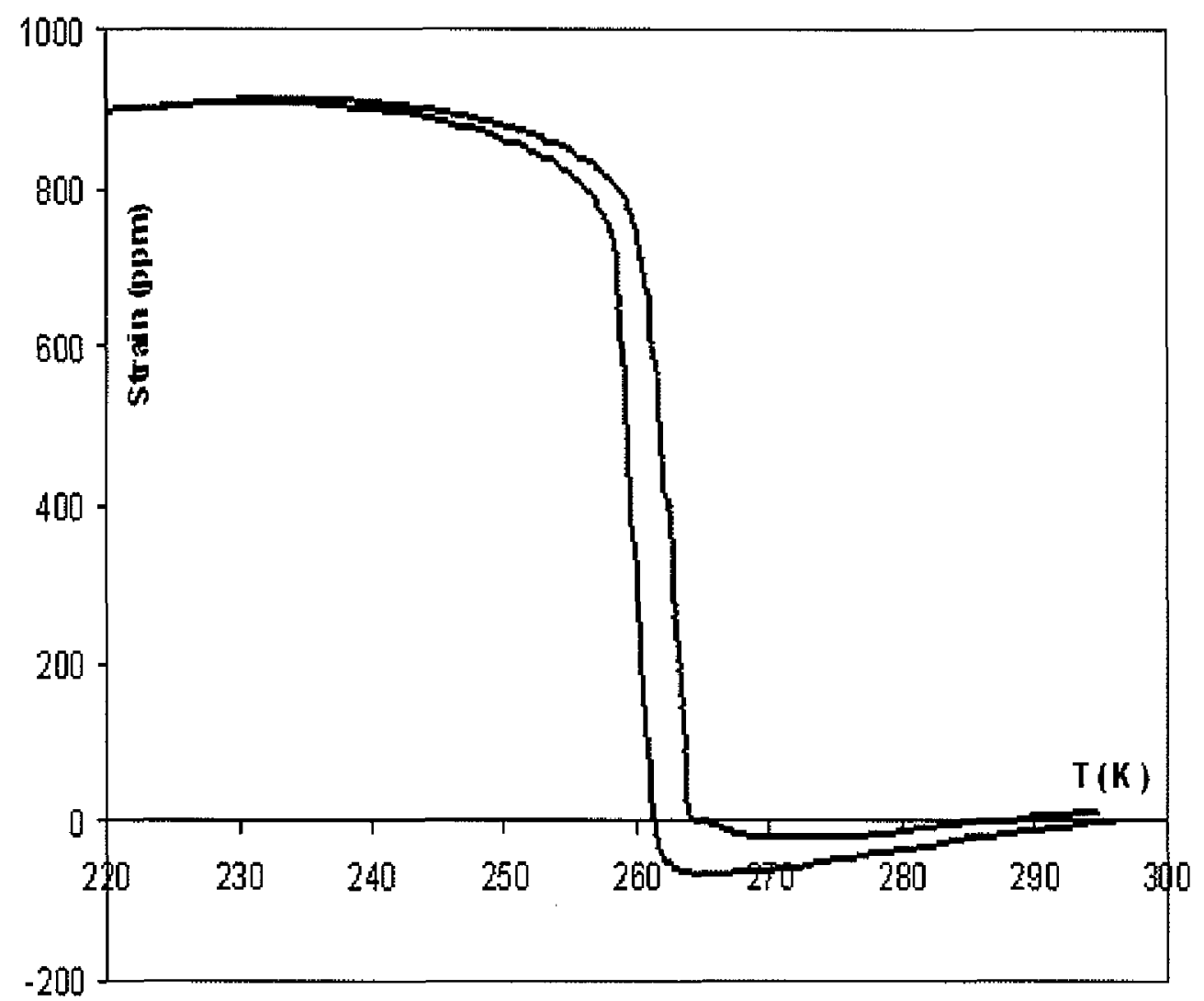

Fig.7-15 Thermal expansion along $b$ axis of single crystal $\mathrm{Gd}_{5}\left(\mathrm{Si}_{2} \mathrm{Ge}_{2}\right)$ under zero magnetic field, $\mathrm{Tc}=259.5 \mathrm{~K}$ on cooling, $\mathrm{Tc}=262.3 \mathrm{~K}$ 


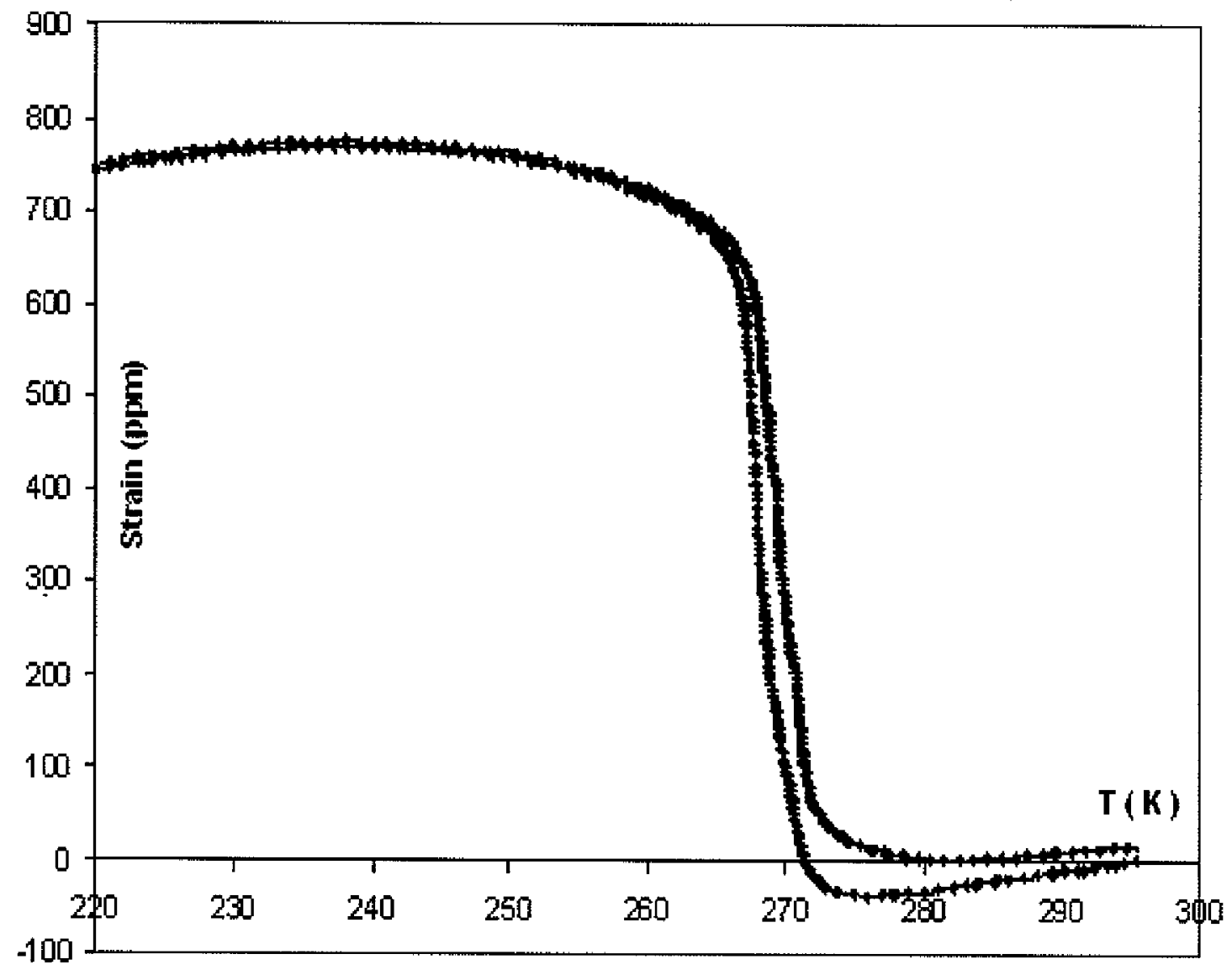

Fig.7- 16 Thermal expansion along $b$ axis of single crystal $\mathrm{Gd}_{5}\left(\mathrm{Si}_{2} \mathrm{Ge}_{2}\right)$ under a magnetic field of 1.5 Tesla 


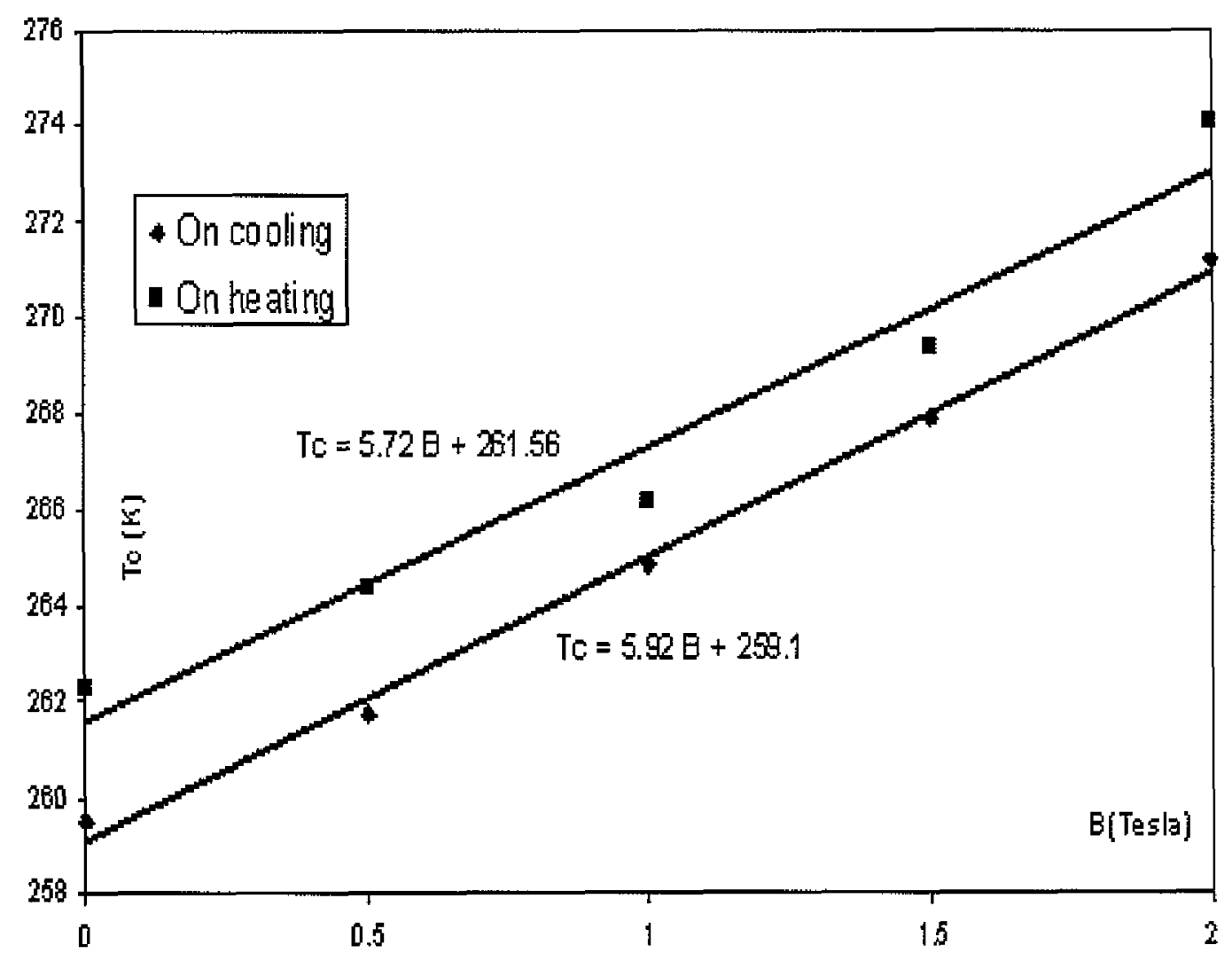

Fig.7- 17 Magnetic field dependence of Curie temperature of single crystal $\mathrm{Gd}_{5}\left(\mathrm{Si}_{2} \mathrm{Ge}_{2}\right)$ along $b$ axis

In order to study whether the magnetic field has different effects on the transition along different principal axes, magnetic fields of different magnitude: $0,0.5,1.0,1.5$, and 2.0 Tesla, were applied along the $\mathrm{b}$ axis. One example was given in Fig.7-16, which is the thermal expansion measurement under the 1.5 Tesla external magnetic field, Tc on cooling is about $268 \mathrm{~K}, \mathrm{Tc}$ on heating is about $270 \mathrm{~K}$. The dependence of Tc on magnetic field were shown in Fig. $7-17$, it is clear that on cooling, $\mathrm{dTc} / \mathrm{dB}$ is about $5.92 \mathrm{~K} / \mathrm{Tesla}$, while $\mathrm{dTc} / \mathrm{dB}$ is about $5.72 \mathrm{~K} /$ Tesla on heating. These data are different from the ones in ref $[36,38]$ and the $\mathrm{dT} / \mathrm{dB}$ value found along the $a$ axis. The reason for it is that $\mathrm{dTc} / \mathrm{dB}$ depends on the alloy composition, the measurement methods, and the way to find the Curie temperature. 


\section{(d) Thermal expansion along the $\mathrm{c}$ axis}

Thermal expansion along the $\mathrm{c}$-axis with $\mathrm{B}=0$ Tesla is shown in Fig 7-18. An abrupt change in strain appears at $\mathrm{T}=259.5 \mathrm{~K}$ on cooling, and at $\mathrm{T}=261.5 \mathrm{~K}$ on heating. It has been verified that this is also due to the so-called first order magneto-crystallographic transformation as found along $a, b$ axis. The Curie temperature has been found to increase with the applied magnetic field at a rate of about $4.9 \mathrm{~K} / \mathrm{T}$ on heating, $4.4 \mathrm{~K} / \mathrm{T}$ on cooling, as shown in Fig. 7-18, 7-19, 7-20.

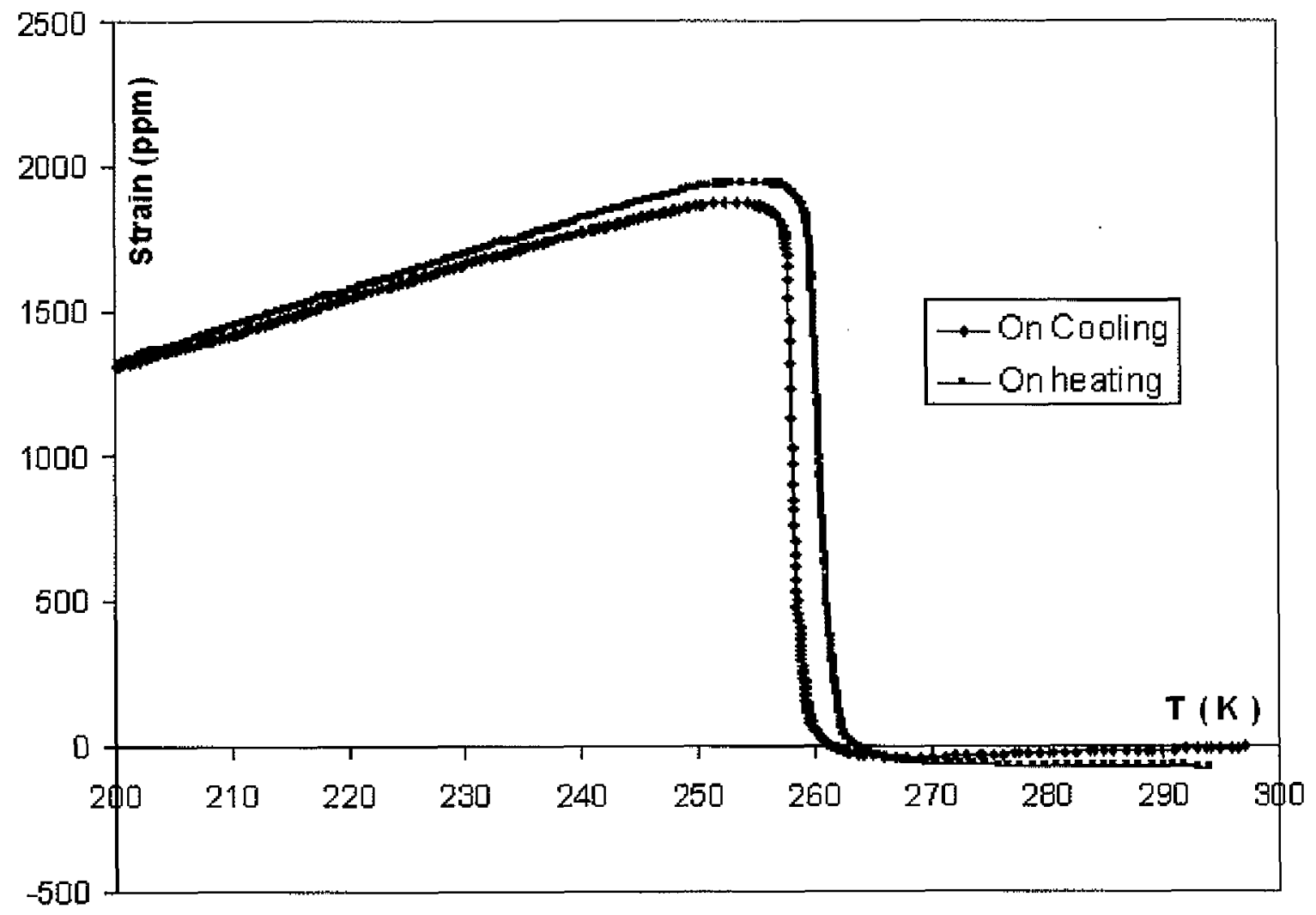

Fig.7-18 Thermal expansion along $\mathrm{c}$ axis for $\mathrm{Gd}_{5}\left(\mathrm{Si}_{2} \mathrm{Ge}_{2}\right)$ with $\mathrm{B}=0$ Tesla 


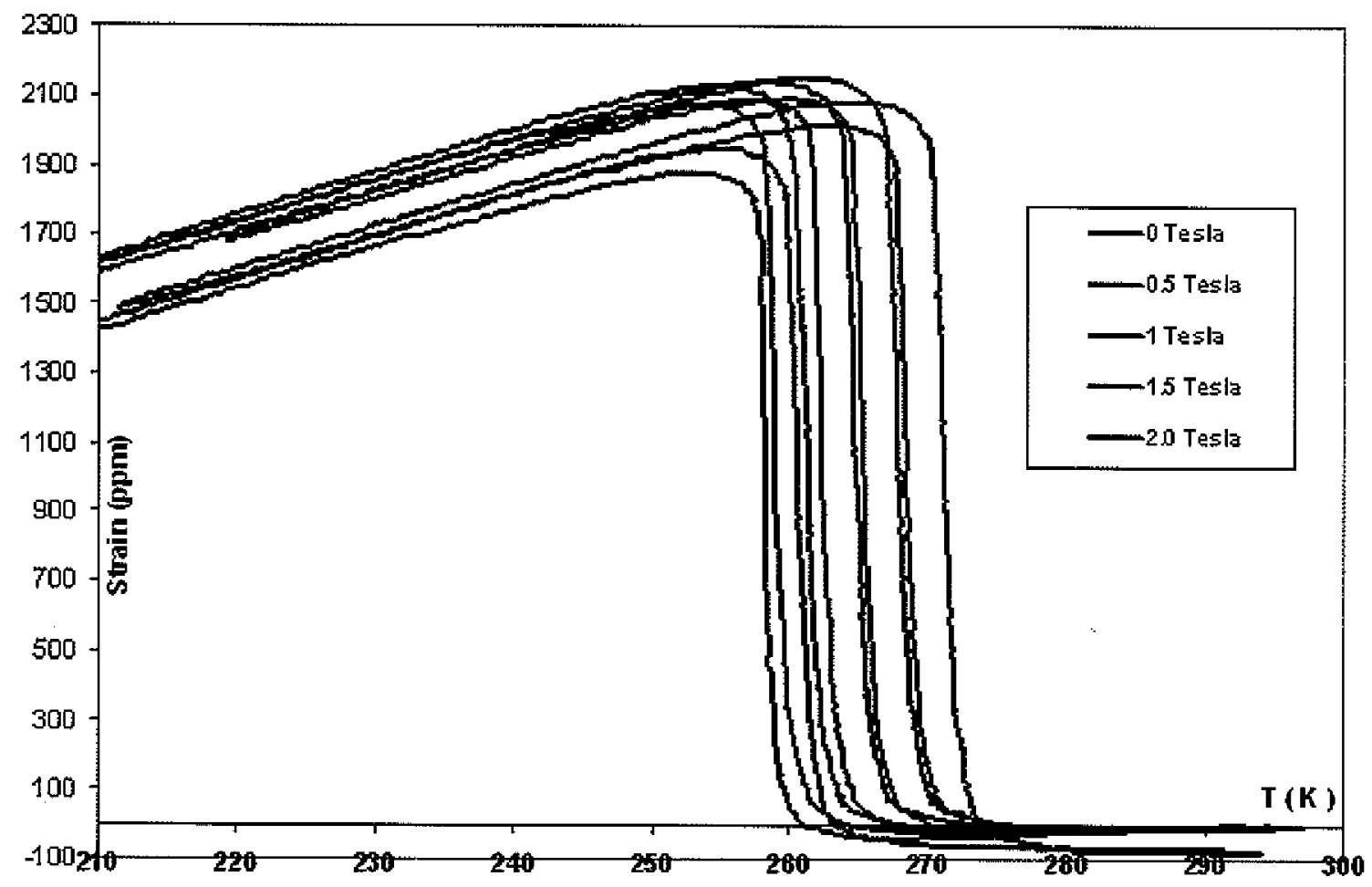

Fig.7-19 Thermal expansion along $c$ axis under different magnetic fields 


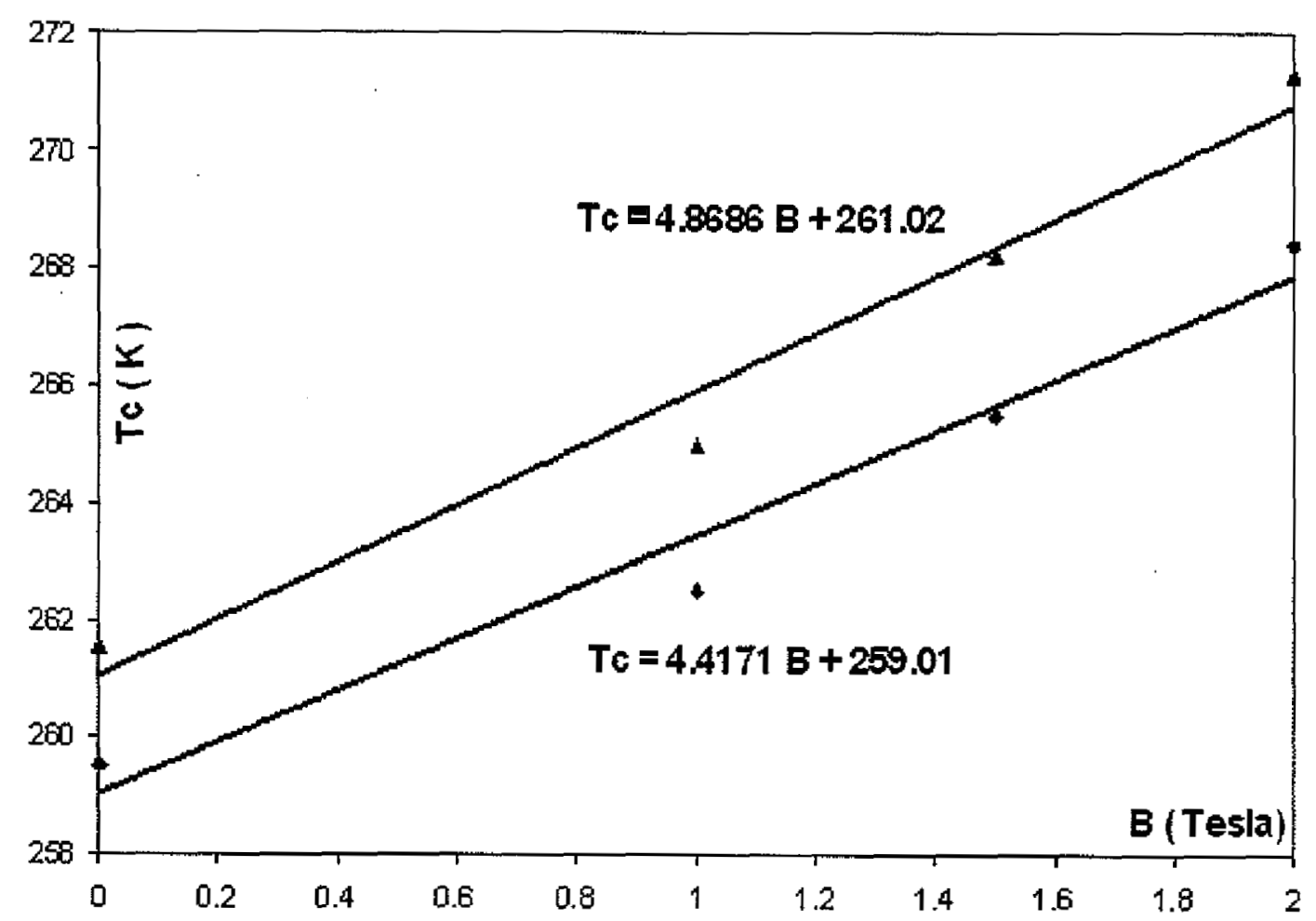

Fig.7- $20 \mathrm{Tc}$ dependence on magnetic field along $\mathrm{c}$ axis of $\mathrm{Gd}_{5}\left(\mathrm{Si}_{2} \mathrm{Ge}_{2}\right)$

\section{(e) Magnetostriction measurements along a, b, $c$ axis:}

Magnetostriction along the $\mathrm{a}, \mathrm{b}$ and $\mathrm{c}$ axes, at temperatures slightly above the transition temperature $(\mathrm{T}>\mathrm{Tc})$, are shown in Fig.7-21, 22, 23. The maximum value is about $-3200 \mathrm{ppm}$ (at $260 \mathrm{~K}$ ), $760 \mathrm{ppm}$ (at $268 \mathrm{~K}$ ), $2100 \mathrm{ppm}$ (at $265 \mathrm{~K}$ ) for a, b, c axis respectively, and showed a very strong anisotropic behavior. These giant magnetostriction values and the signs are in consistent with the thermal expansion experimental results. 


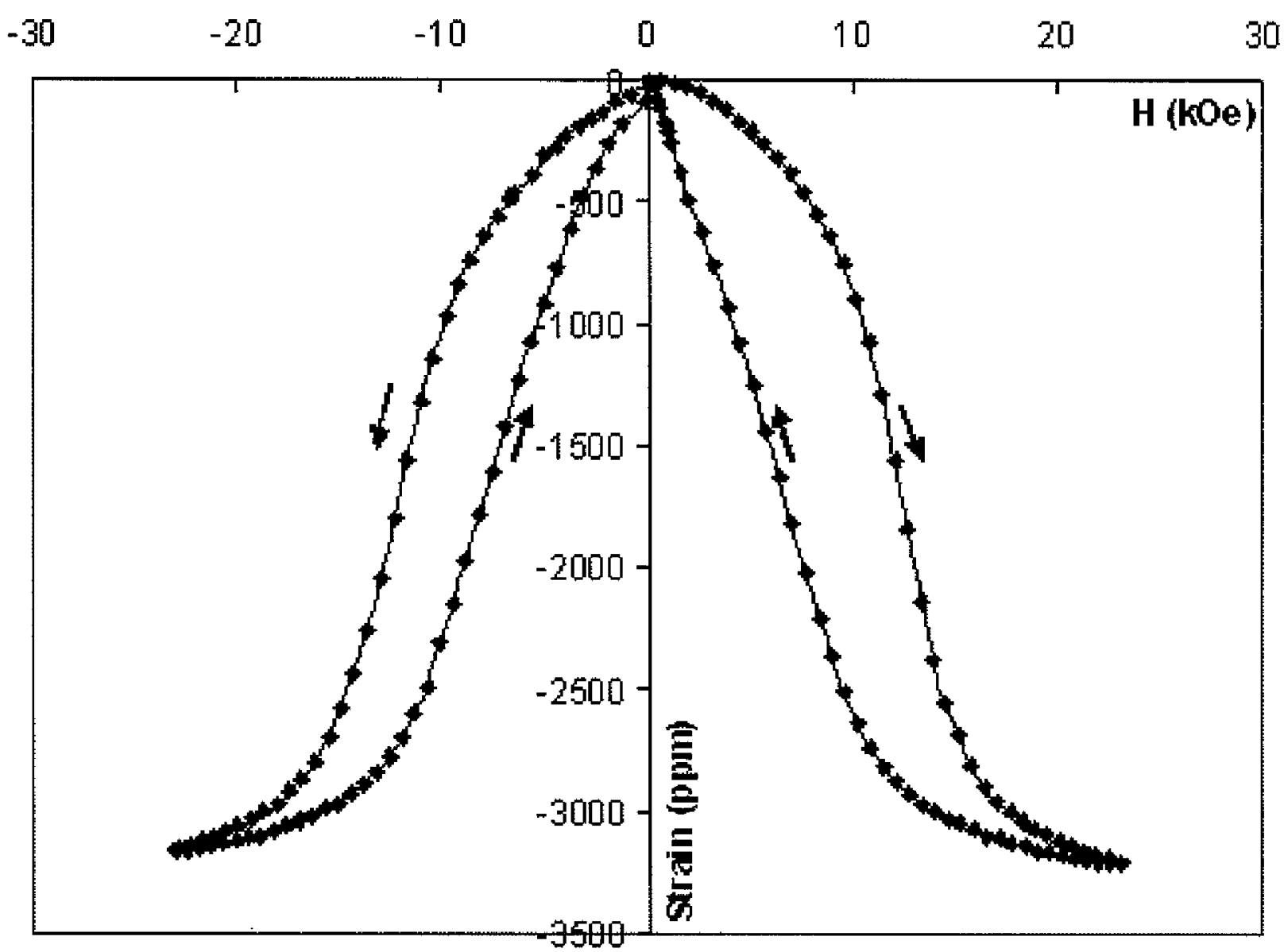

Fig.7-21 Magnetostriction along a axis at $\mathrm{T}=260 \mathrm{~K}$ for $\mathrm{Gd}_{5}\left(\mathrm{Si}_{2} \mathrm{Ge}_{2}\right)$ 


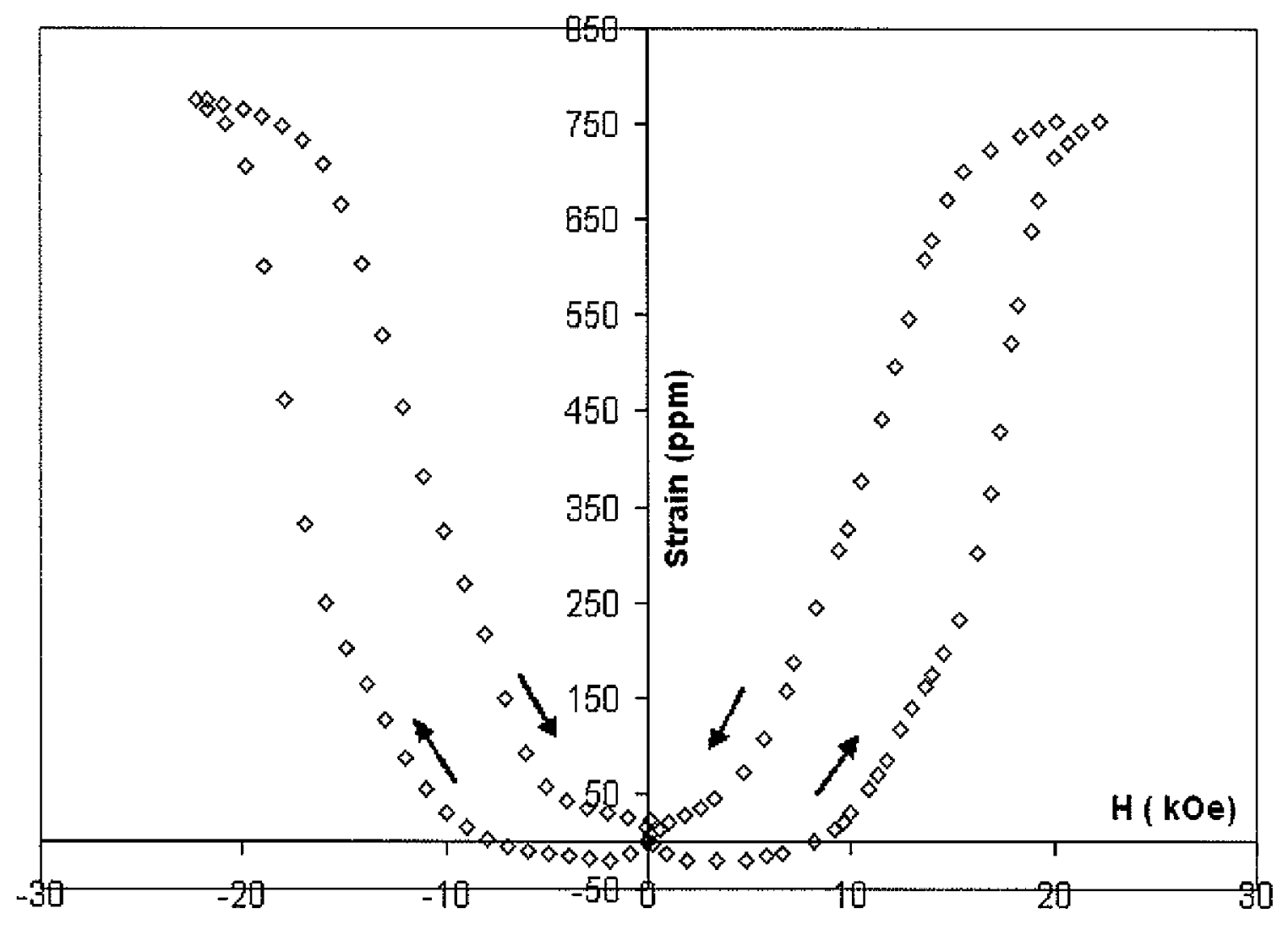

Fig.7-22 Magnetostriction along $b$ axis of single crystal $\mathrm{Gd}_{5}\left(\mathrm{Si}_{2} \mathrm{Ge}_{2}\right)$ at $\mathrm{T}=268 \mathrm{~K}$ 


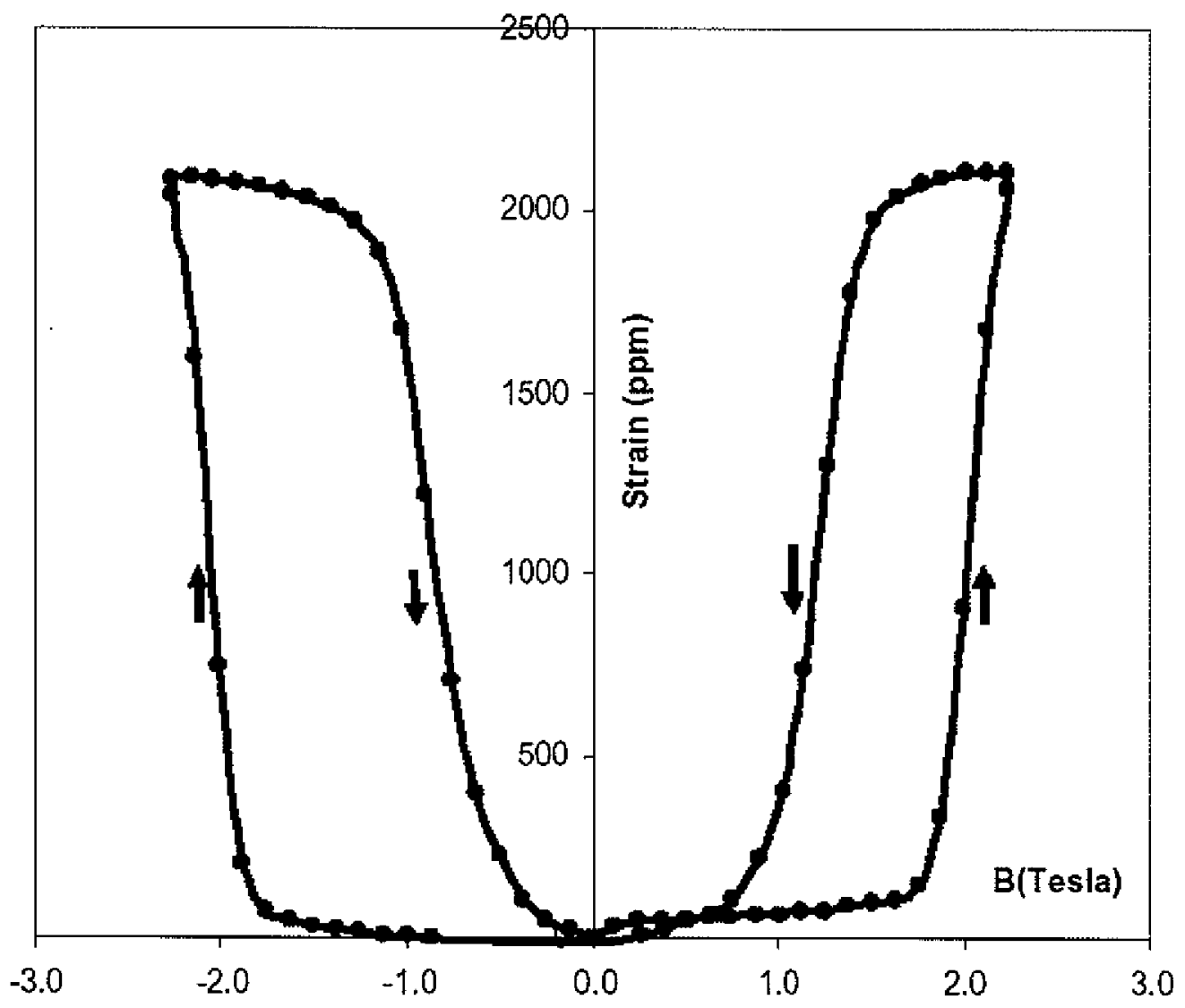

Fig.7-23 Magnetostriction measurement along c axis for $\mathrm{Gd}_{5}\left(\mathrm{Si}_{2} \mathrm{Ge}_{2}\right)$ at $\mathrm{T}=265 \mathrm{~K}$

Compared to the magnetostriction measurement at temperatures below the transition temperature $(\mathrm{T}<\mathrm{Tc})$, it was found that the magnetostriction values along three principal axes were much smaller, see Fig.7-24, 25, 26 at $\mathrm{T}=180 \mathrm{~K}$ ( a axis), $\mathrm{T}=214 \mathrm{~K}$ ( $\mathrm{b}$ axis), $\mathrm{T}=258$ $\mathrm{K}$. The explanation is that the magnetic field shifts the transition temperature to higher temperatures, so that no magneto-structural first order transition occurred, only field-induced magnetostriction was observed. 
It is well known that magnetostriction is a response of a magnetic material to the magnetic field, and is normally due to spin-orbit coupling, unless as in this case, it is due to a magnetostructural transformation. From the above results, we know that $\mathrm{Gd}_{5}\left(\mathrm{Si}_{2} \mathrm{Ge}_{2}\right)$ has two magnetic states with two crystal structures coupled tightly together. For instance, magnetostriction measurements along the $\mathrm{b}$ axis, at $214 \mathrm{~K}$ and zero field, the sample is in a ferromagnetic and orthorhombic state, the observed magnetostriction is due to the domain structures reconfiguration, such as non- $180^{\circ}$ domain wall movement, and the magnetization rotation in each domain. Generally, it is classified as a field-induced magnetostriction. At 214 $\mathrm{K}$, there is no structural transition making contribution to the observed strain value. Besides, the positive field magnetostriction value found in Fig. $7-24$ along the a axis and at $\mathrm{T}=180 \mathrm{~K}$ also explains the thermal expansion along the a axis at temperatures below $\mathrm{Tc}$ which decreases with increasing applied magnetic fields as shown in Fig.7-13.

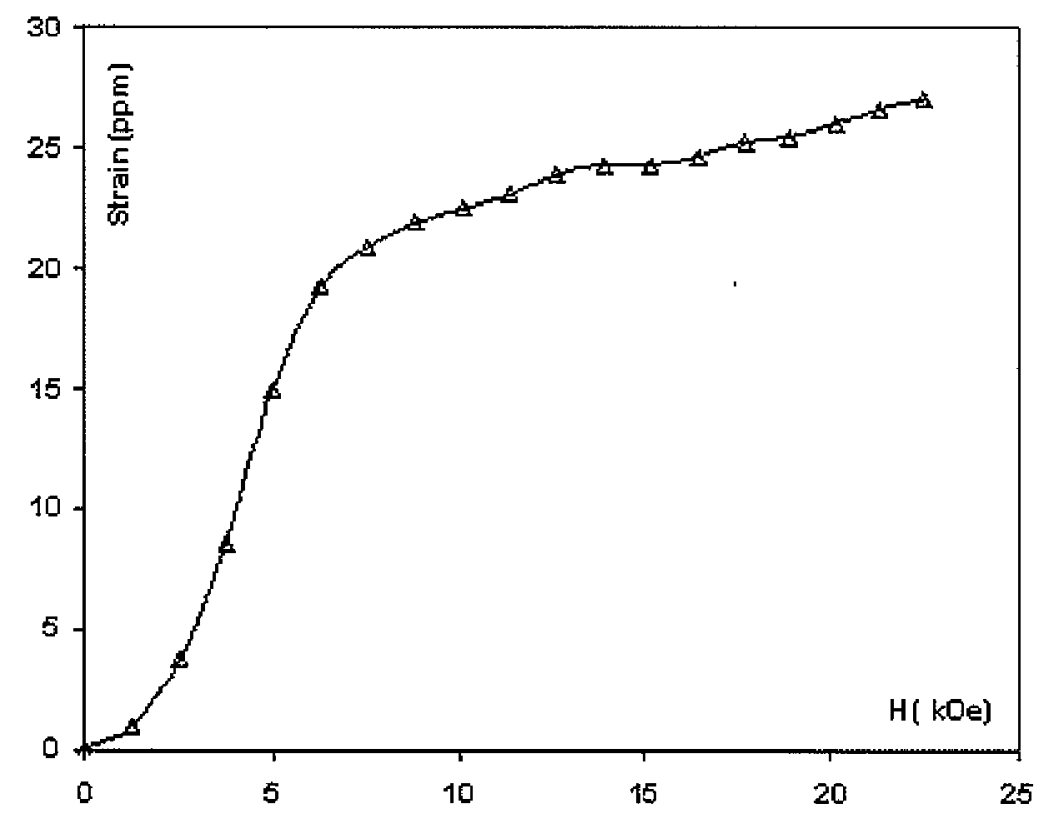

Fig.7-24 Magnetostriction along a axis of single crystal $\mathrm{Gd}_{5}\left(\mathrm{Si}_{2} \mathrm{Ge}_{2}\right)$ at $\mathrm{T}=180 \mathrm{~K}$ 
The observed magnetostriction of the material when it remains wholly in its ferromagnetic state is small (about $18 \mathrm{ppm}$ ) and this is shown in Fig.7-25. However, at $268 \mathrm{~K}$ and zero magnetic fields, the material can be driven across the transition from a paramagnetic and monoclinic state to a ferromagnetic and orthorhombic state. Since the transition temperature can be shifted by magnetic field, therefore, during the magnetostriction measurement process, the applied magnetic field can move the transition temperature across $268 \mathrm{~K}$ back and forth, resulting in adjacent slabs shear along a axis, and $\mathrm{Si}(\mathrm{Ge})-\mathrm{Si}(\mathrm{Ge})$ bonds breaking and reforming, which is believed to be responsible for the observed large strain, about $770 \mathrm{ppm}$.

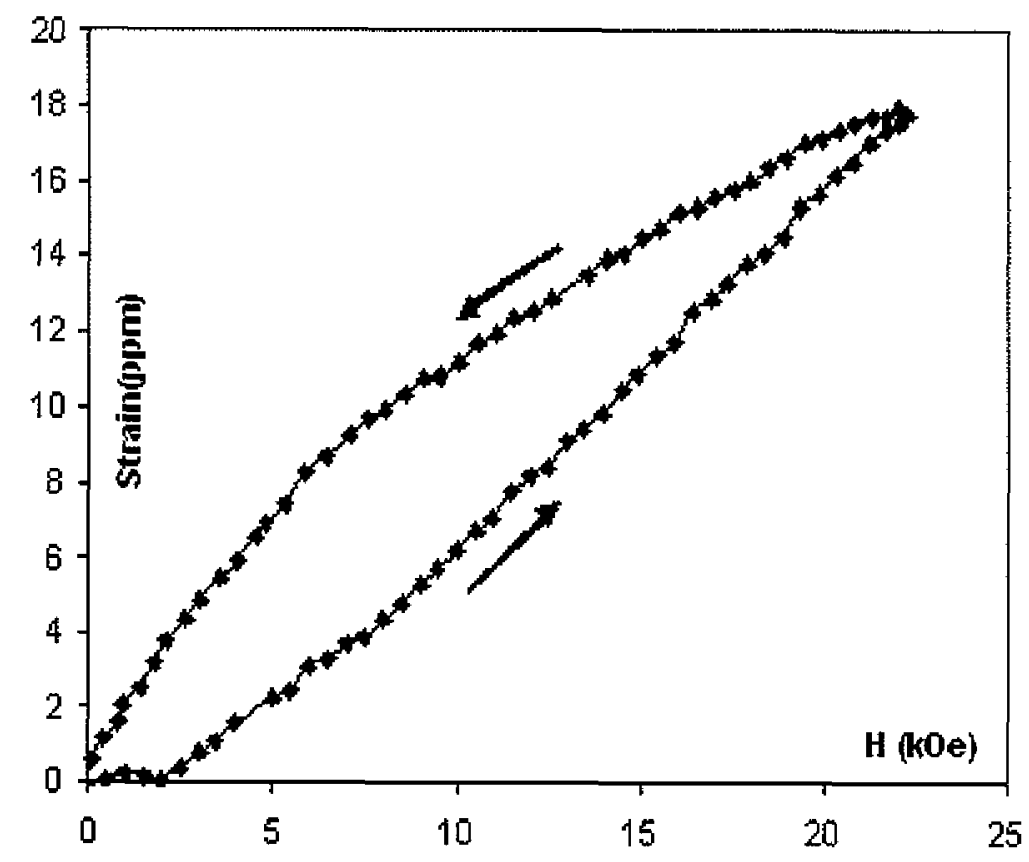

Fig.7- 25 Magnetostriction along b axis of single crystal $\mathrm{Gd}_{5}\left(\mathrm{Si}_{2} \mathrm{Ge}_{2}\right)$ at $\mathrm{T}=214 \mathrm{~K}$ 


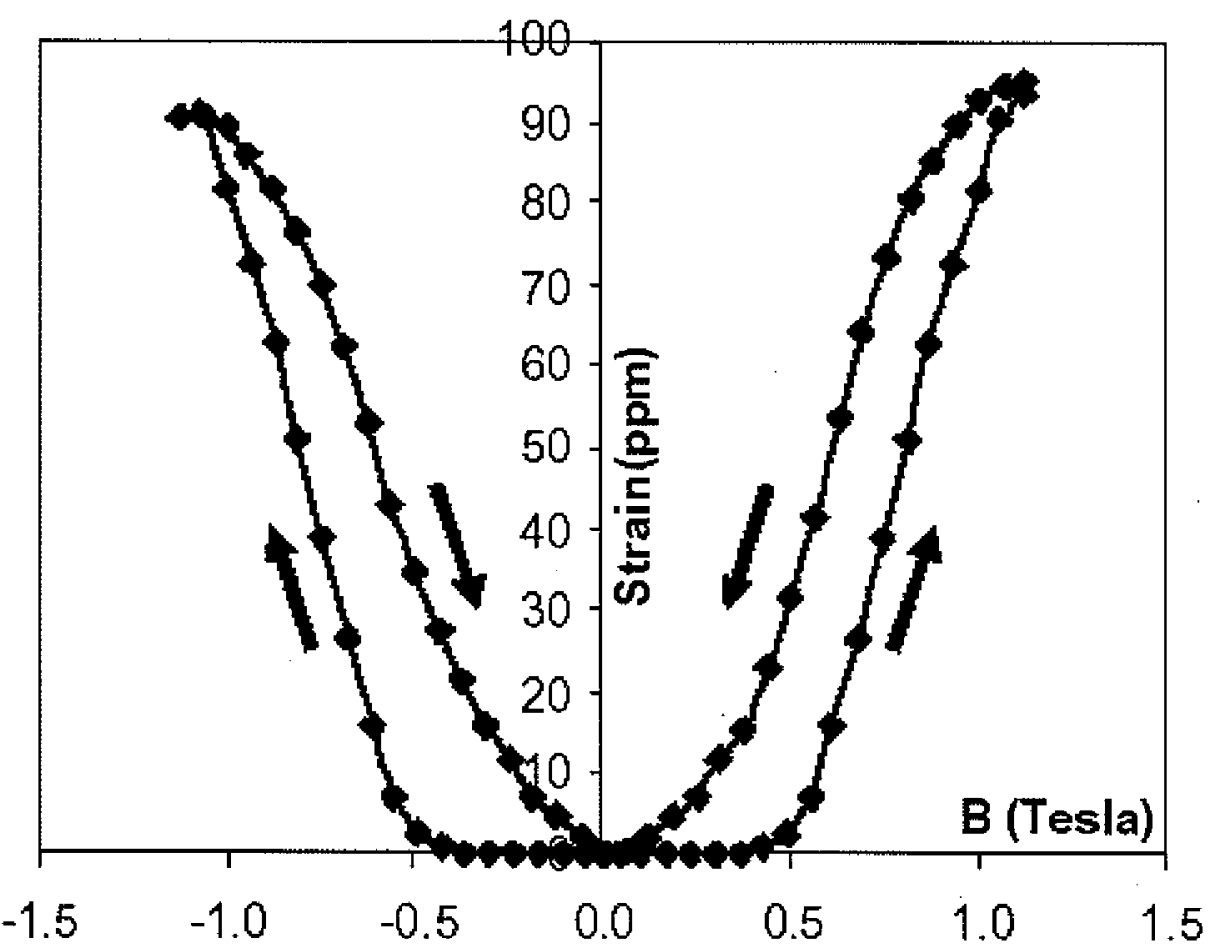

Fig.7-26 Magnetostriction measurement along $\mathrm{c}$ axis for $\mathrm{Gd}_{5}\left(\mathrm{Si}_{2} \mathrm{Ge}_{2}\right)$ at $\mathrm{T}=258 \mathrm{~K}$

Similar results have been found for $\mathrm{Gd}_{5}\left(\mathrm{Si}_{0.1} \mathrm{Ge}_{0.9}\right)_{4}$ by Morellon, et al [36]. $\mathrm{Gd}_{5}\left(\mathrm{Si}_{0.1} \mathrm{Ge}_{0.9}\right)_{4}$ exhibits a first order transition at $\mathrm{Tc}=81 \mathrm{~K}$ (when $\mathrm{B}=0$ Tesla), and a second order transition at $\mathrm{T}_{\mathrm{N}}=127 \mathrm{~K}$. Below its Curie temperature, it was found there was no significant magnetostriction in a fields of up to 12 Tesla. At $\mathrm{T}=110 \mathrm{~K}$, which is within the range of $\mathrm{Tc}<$ $\mathrm{T}<\mathrm{T}_{\mathrm{N}}$, it required application of an 8 Tesla magnetic field in order to produce giant magnetostriction.

The thermal expansion under zero field along three axes are shown in Fig.7-13, 7-15, 7-18. The maximum strains are $-5200,900,2000$ ppm along a, b, c axis respectively. The thermal expansion under different magnetic fields along $a, b, c$ axis are shown in Fig.7-13, 7-16, 7-19. All these figures show that the transition is a first order transition, and that the transition 
temperature can be shifted by magnetic fields, but with different rates along different axis, as shown in Fig.7-14, 17, 20. In addition, from Figs. 7-13, 7-15, 7-18, it can seen that below Tc the strain change vs. temperature along the $a$ axis is the largest, while along the $b$ and $c$ axes it is much smaller. Another observation is that at the transition temperature, the strain change along the $a$ axis has a negative value, while it has a positive value along the $b$ and $c$ axes. It is the result of a combination of structural change and appearance/disappearance of spontaneous magnetization and magnetostriction at the Curie point.

\section{Conclusions}

The unusual isothermal magnetization behavior along $\mathrm{a}, \mathrm{b}$ and $\mathrm{c}$ axis at $265 \mathrm{~K}$ shows the coexistence of paramagnetic and ferromagnetic phases, and the fact that the $b$ axis is the easy axis. All of the thermal expansion measurements along a, b, c axis show that the Curie point transformation is of the first order type, and can be triggered by external magnetic field, but with a different $\mathrm{dT} / \mathrm{dB}$ rates along different axis. Giant magnetostriction found along $a, b, c$ axis was only observed at the vicinity of and slightly above the Curie temperature ( $T>T c$ ), it was believed that this was due to the first order magneto-crystalline phase transformation. While the magnetostriction measured at $\mathrm{T}<\mathrm{Tc}$ was found to be very small compared to the giant magnetostriction found at $\mathrm{T}>\mathrm{Tc}$. The values and signs of thermal expansion and magnetostriction measurement along three axes and magnetization measurements show strongly anisotropic properties.

\section{Three polycrystalline samples $\mathrm{Gd}_{5}\left(\mathrm{Si}_{2.09} \mathrm{Ge}_{1.91}\right)$}

\section{(a) Prepared by arc melting method}


For these two polycrystalline samples, thermal expansion measurement results are shown in Fig.7-27 for the sample $\mathrm{Gd}_{5}\left(\mathrm{Si}_{2,09} \mathrm{Ge}_{1.91}\right)$ made from $\mathrm{AL} \mathrm{Gd}$ at $\mathrm{B}=0$ and $1 \mathrm{~T}$. An abrupt change in strain was observed at $283 \mathrm{~K}$ on cooling and $288 \mathrm{~K}$ on heating for $\mathrm{B}=0 \mathrm{~T}$; and at $287.5 \mathrm{~K}$ on cooling and $298 \mathrm{~K}$ on heating for $\mathrm{B}=1 \mathrm{~T}$. A discontinuity in thermal strain and thermal hysteresis are signatures of a first-order phase transition. Above the transition temperature, the sample is paramagnetic and monoclinic. Below the transition temperature, it is ferromagnetic and orthorhombic. Previous references have indicated that this first order coupled magnetic-crystallographic transition only occurs within the composition range 0.24 $\leqq \mathrm{x} \leqq 0.5$ [15]. Recently, a more complete magnetic and crystallographic phase diagram has been established for the $\mathrm{Gd}_{5}\left(\mathrm{Si}_{\mathrm{x}} \mathrm{Ge}_{1-\mathrm{x}}\right)_{4}$ system [14], where a two-phase region $(0.503<\mathrm{x}$ $<0.575)$ is added between two regions that show a single phase around room temperature.

The composition of the present samples $(x=0.5225)$ falls into this so-called two-phase region. For this composition the two crystallographic structures that can exist around room temperature are: monoclinic $\mathrm{Gd}_{5} \mathrm{Si}_{2} \mathrm{Ge}_{2}$-type paramagnetic state (the $\beta$ phase) which is formed above $1200 \mathrm{~K}$ and can be retained at room temperature, and orthorhombic $\mathrm{Gd}_{5} \mathrm{Si}_{4}{ }^{-}$ type paramagnetic state (the $\gamma$ phase) which is formed on heat treating the $\beta$ phase above 690 $\mathrm{K}$ but below $1200 \mathrm{~K}$ and which appears to be stable at room temperature. When the paramagnetic $\beta$ phase is cooled down below its Curie temperature, a coupled first-order magnetic-crystallographic phase transition occurs, and the ferromagnetically ordered $\mathrm{Gd}_{5} \mathrm{Si}_{4}{ }^{-}$ type structure ( $\alpha$ phase) is formed. This transformation is reversible. When the paramagnetic orthorhombic $\mathrm{Gd}_{5} \mathrm{Si}_{4}$-type $\gamma$-phase is cooled, it undergoes only an ordinary second-order 
transition from paramagnetic to ferromagnetic with no associated crystallographic change. This transition is also reversible. In the two-phase region, one can get either or both of $\beta$ or $\gamma$ phase to exist at room temperature, depending on heat treatment.

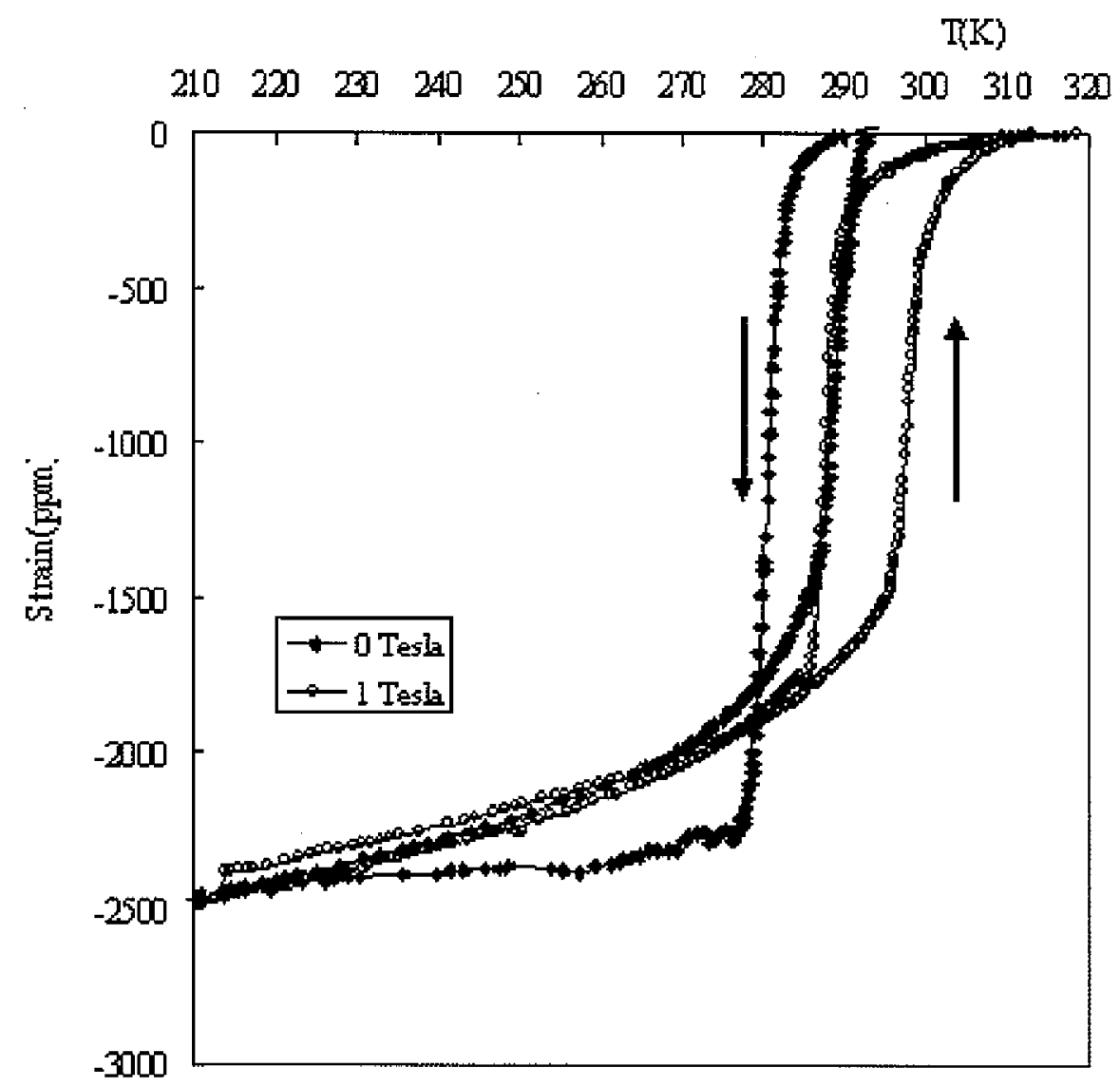

Fig.7-27 Linear thermal strain (under fields of 0 and $1 \mathrm{~T}$ ) of polycrystalline $\mathrm{Gd}_{5} \mathrm{Si}_{2.09} \mathrm{Ge}_{1.91}$ made from Ames Laboratory high purity gadolinium. 


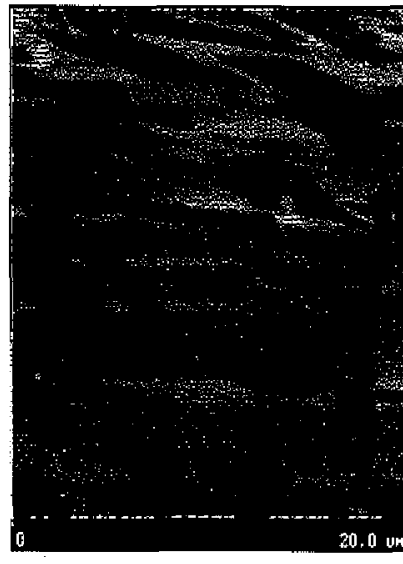

$285.7 \mathrm{~K}$

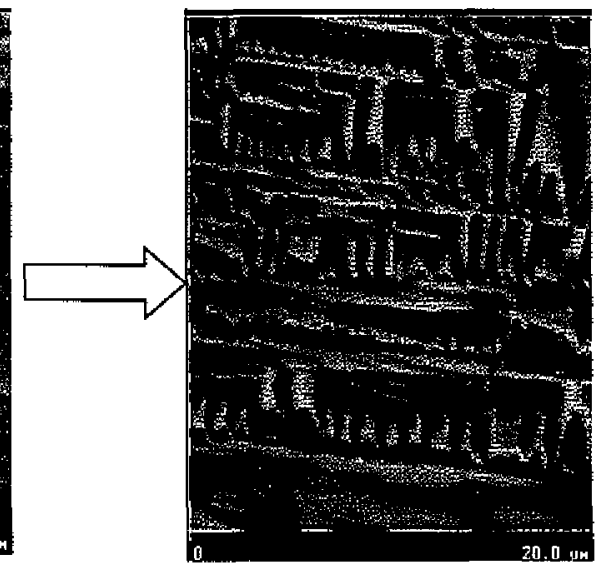

$283.9 \mathrm{~K}$

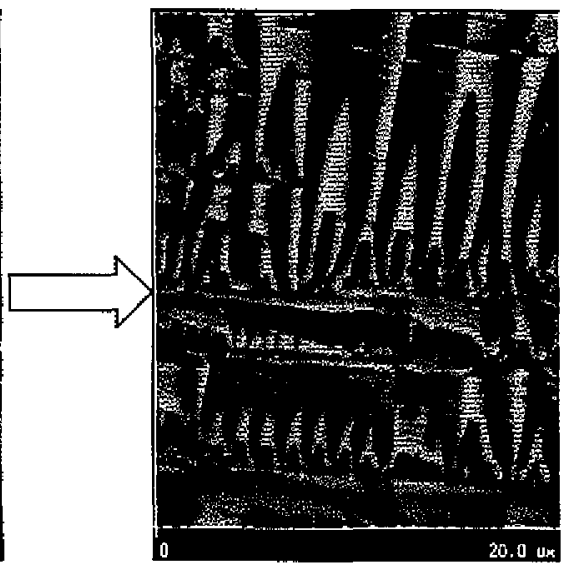

$281.7 \mathrm{~K}$

Fig.7-28. MFM images showing the magnetic phase transformation process for the sample made with high purity Ames Laboratory gadolinium. The leftmost image shows the sample in the paramagnetic state, the middle and the rightmost images show the ferromagnetic state with increasing domain size.

The present samples were heat treated to ensure single phase before they were used for thermal expansion measurement. In Fig.7-28, the MFM images show the transition from paramagnetic state to ferromagnetic state upon cooling, and when combined with the results in Fig.7-27 which shows a discontinuity in thermal strain at transition temperature of $283 \mathrm{~K}$ on cooling and at $288 \mathrm{~K}$ on heating, and hysteresis in the Curie temperature, these are indicative of a first order phase transition. Thus taken together, they appear to indicate a firstorder simultaneous magnetic-crystallographic phase transition. 
The $\gamma$ phase of $\mathrm{Gd}_{5} \mathrm{Si}_{4}$ is ferromagnetic at room temperature and therefore not subject to a phase transformation upon cooling. Therefore on the basis of these results, it is believed that at room temperature, these samples adopted the crystal structure of the $\beta$ phase. In addition,

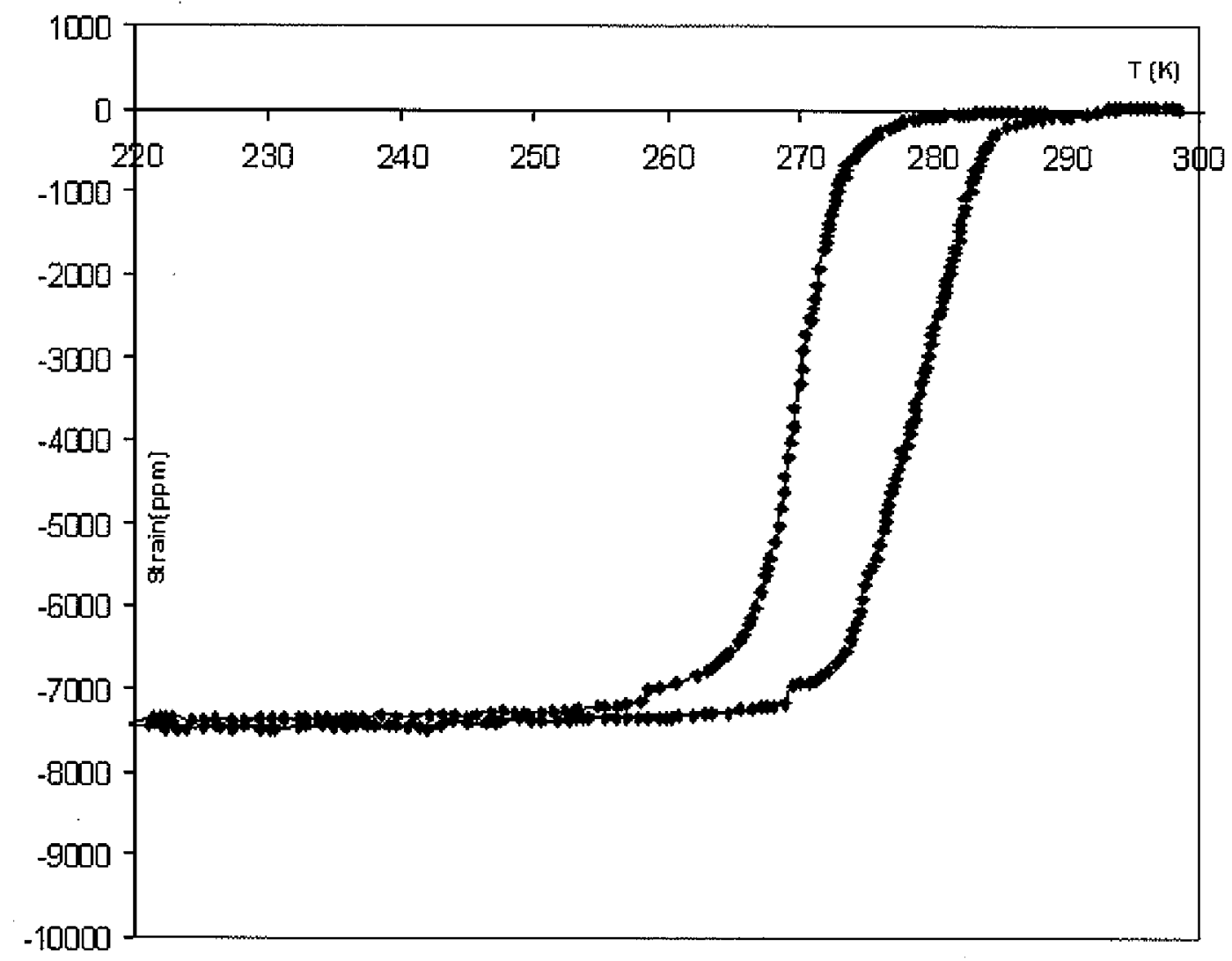

Fig.7-29 Linear thermal strain in zero applied field of polycrystalline $\mathrm{Gd}_{5} \mathrm{Si}_{2.09} \mathrm{Ge}_{1.91}$ made from commercial quality gadolinium.

The magnetic field can shift the Curie point to higher temperatures which also demonstrates that this first order transformation can be triggered by either temperature or applied magnetic field. In Fig.7-27, the linear thermal expansion $(\Delta / / /)$ of $0.26 \%$, corresponds to a volume expansion of $(\Delta V / V=3 \Delta l / l)$ about $0.78 \%$, assuming minimal texturing. The thermal expansion results for sample 2 made from commercial Gd are shown in Fig.7-29. On cooling 
$\mathrm{T}_{\mathrm{c}}=270 \mathrm{~K}$, and on heating $\mathrm{T}_{\mathrm{c}}=275 \mathrm{~K}$. Thus the higher levels of impurities in the commercial Gd appear to have the effect of decreasing the transition temperature by $\Delta \mathrm{T}_{\mathrm{c}}=$ $13 \mathrm{~K}$ (on either cooling or heating). The linear thermal expansion was $0.85 \%$, corresponding to volume expansion of $2.55 \%$, assuming minimal texturing. These values are considerably higher than those obtained in the sample made with high-purity Gd.

\section{(b) Prepared by induction melting method}

For this sample, thermal expansion was conducted first in a zero magnetic field; the results were shown in the Fig.7-30. It is obvious that there also exist a temperature hysteresis during the cooling and heating process. It is the fingerprint of a first order transition. According to the ref [17], for $\mathrm{Gd}_{5}\left(\mathrm{Si}_{2.09} \mathrm{Ge}_{1.91}\right)$, it is in the middle region, where on cooling, the sample was transformed from a monoclinic crystal structure and a paramagnetic state into an orthorhombic and ferromagnetic state. On heating, the sample was transformed from an orthorhombic and ferromagnetic state into a monoclinic crystal structure and a paramagnetic state. The discontinuous changes in strain found in Fig.7-30 show such a simultaneous magnetic and structural transformation. From this figure, it is shown that the transition temperature is $275.5 \mathrm{~K}$ on cooling, $278 \mathrm{~K}$ on heating, and a magnetic/structural transformation can induce a huge strain, about $7350 \mathrm{ppm}$, contracting on cooling, and expanding on heating. Our previous studies on polycrystal $\mathrm{Gd}_{5}\left(\mathrm{Si}_{2.09} \mathrm{Ge}_{1.91}\right)$ sample with different grade starting material Gd showed that the Curie temperature was lower and the thermally-induced strain higher, in the sample made from lower purity level Gd starting materials compared with the sample made from high purity Gd metal [40]. 


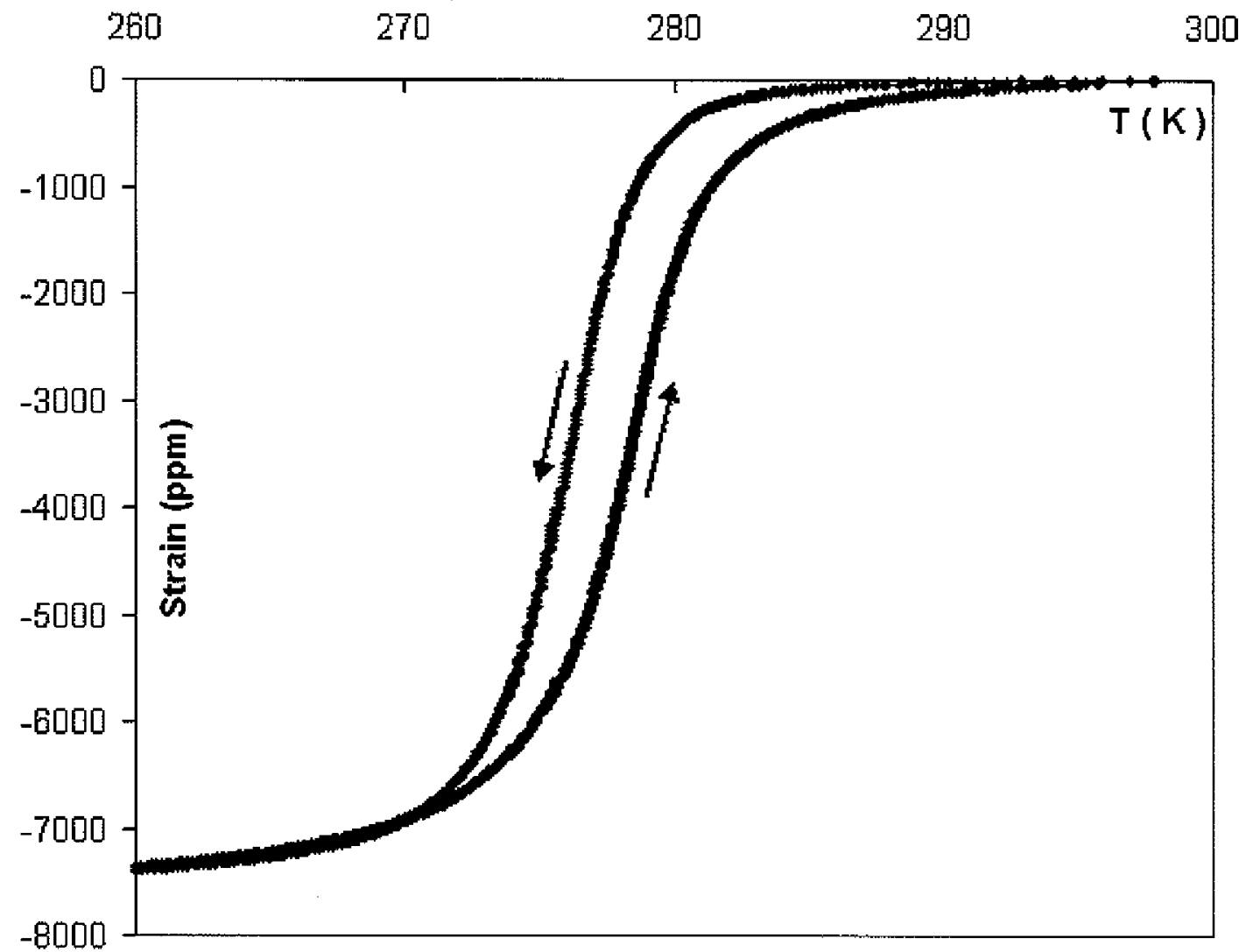

Fig.7-30 Thermal expansion for polycrystal sample $\mathrm{Gd}_{5}\left(\mathrm{Si}_{2.09} \mathrm{Ge}_{1.91}\right)$ under zero magnetic field, $\mathrm{Tc}=275.5 \mathrm{~K}$ on cooling, $\mathrm{Tc}=278 \mathrm{~K}$ on heating

The result in Fig. 7-30 shows the thermal expansion obtained with commercial grade Gd starting material, but with even higher transition temperature (in Fig.7-29, $\mathrm{T}_{\mathrm{c}}=270 \mathrm{~K}$ on cooling, $T_{c}=275 \mathrm{~K}$ on heating), and a smaller temperature hysteresis of $2 \mathrm{~K}$ compared to the $5 \mathrm{~K}$ found elsewhere. Therefore, a conclusion can be drawn that the first order transition also depends on the preparation method used. It could be that these two methods result in different weight loss, or different grain size. Grain size has no effect on transition temperature, it usually affects the coercivity of magnetic materials [4]. 
This also confirms that the results found previously that the Curie temperature of the polycrystal sample $\mathrm{Gd}_{5}\left(\mathrm{Si}_{2.09} \mathrm{Ge}_{1.91}\right)$ with commercial grade $\mathrm{Gd}$ (96.9 at.\% pure) starting material is lower than that of high purity Gd metals ( $99.8 \%$ pure) made by Ames Laboratory.

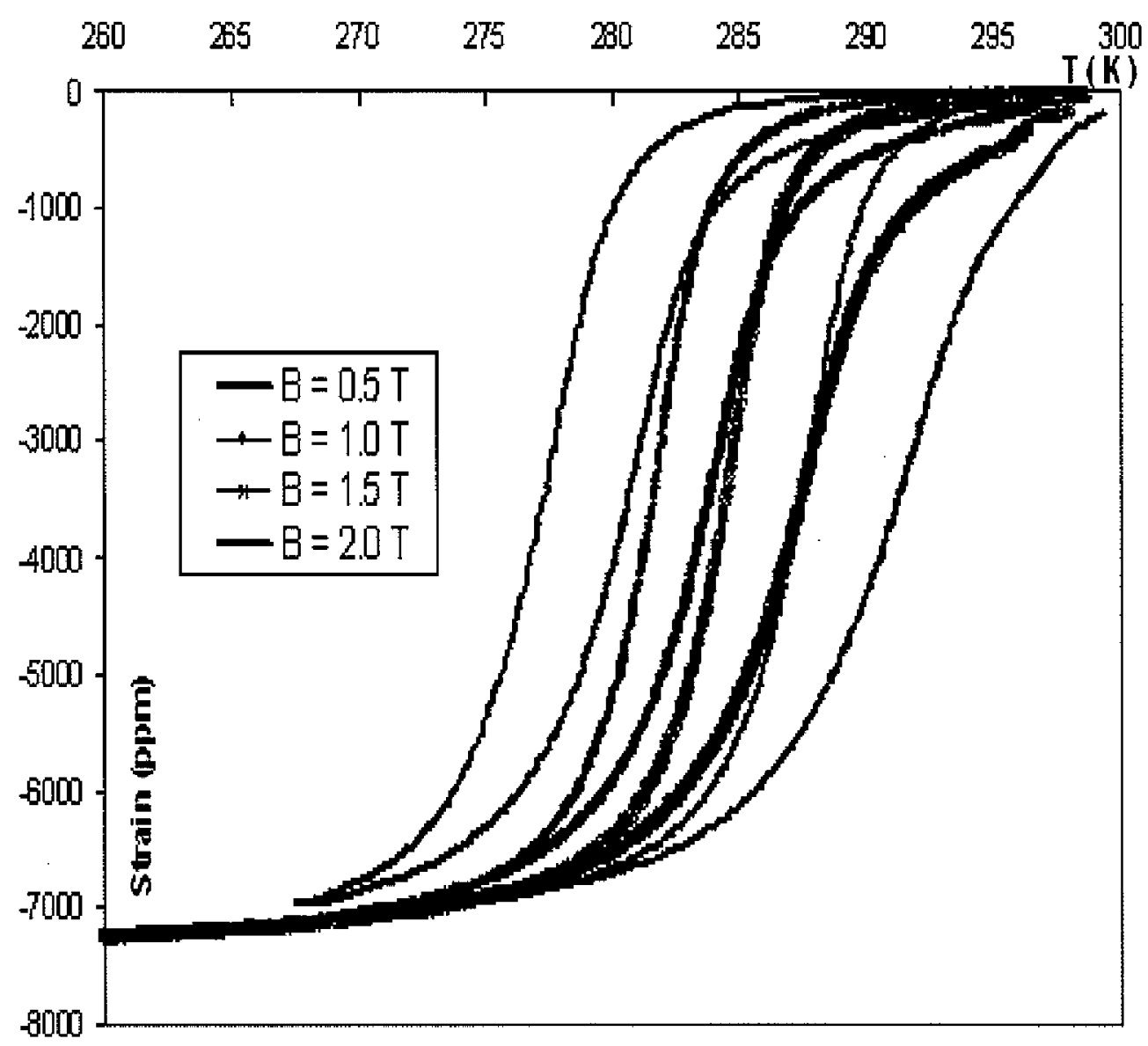

Fig.7-31 Thermal expansion measurement for polycrystal sample $\mathrm{Gd}_{5}\left(\mathrm{Si}_{2.09} \mathrm{Ge}_{1.91}\right)$ under 0.5, $1.0,1.5,2.0$ Tesla

Thermal expansion measurements have also been conducted under different strength magnetic fields. The results are shown in Fig.7-31. For this composition an unusual behavior was found that the first order transformation transition depends linearly on the applied magnetic field, as shown in Fig.7-32, for 0.5, 1.0, 1.5 and 2.0 Tesla, on cooling Tc is $277.3 \mathrm{~K}$, 
$281.5 \mathrm{~K}, 284.4 \mathrm{~K}$ and $287.4 \mathrm{~K}$ respectively, on heating $\mathrm{Tc}$ is $280.7,283.7,287.3,291.2 \mathrm{~K}$ respectively. The change on cooling is about $6.18 \mathrm{~K} /$ Tesla, whereas on heating, it is about 6.6 K/Tesla.

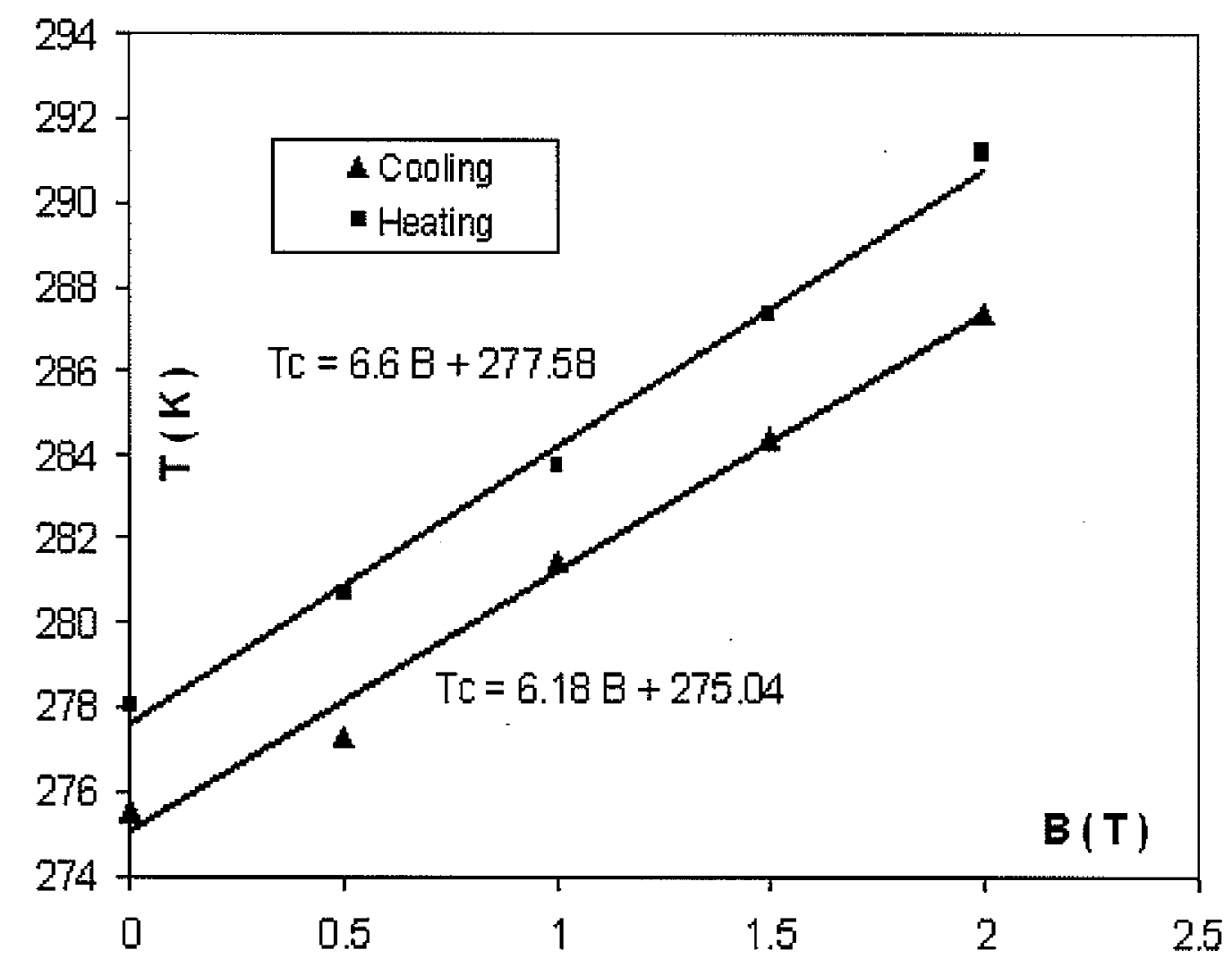

Fig.7-32 Dependence of Curie temperature of polycrystal sample $\mathrm{Gd}_{5}\left(\mathrm{Si}_{2,09} \mathrm{Ge}_{1.91}\right)$ on the applied magnetic field, and transformation directions

Magnetostriction measurements were performed within $|\mathrm{H}|=10 \mathrm{kOe}, 33 \mathrm{kOe}$ at $240 \mathrm{~K}, 285$ $\mathrm{K}$ respectively, as shown in Fig.7-33, 34. At $240 \mathrm{~K}$, which is in the ferromagnetic and orthorhombic state and is below the transition temperature, the applied magnetic field was found to increase the Curie temperature, and the transition temperature would not be reached during the magnetostriction process. Therefore, the first order magnetic-structural transition 
has not been triggered; the observed strain is a field induced magnetostriction, which is only about $40 \mathrm{ppm}$.

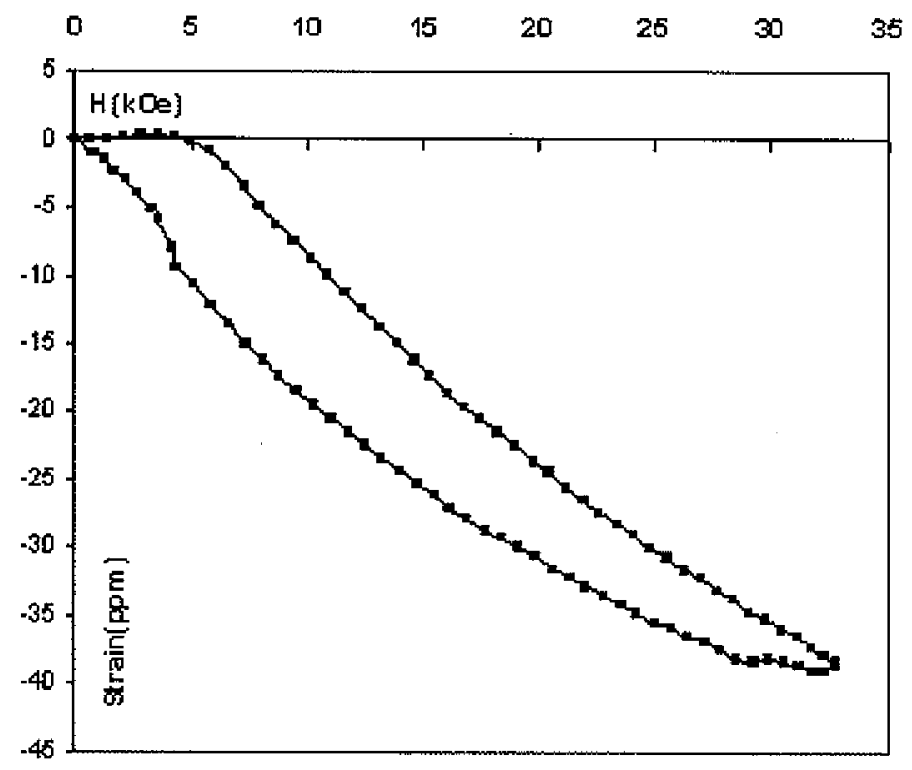

Fig.7-33 Magnetostriction measurement for polycrystal sample $\mathrm{Gd}_{5}\left(\mathrm{Si}_{2.09} \mathrm{Ge}_{1.91}\right)$ at $240 \mathrm{~K}$

However, when magnetostriction was conducted at $285 \mathrm{~K}$, which is in the vicinity of the transition temperature found under zero magnetic field, say $275.5 \mathrm{~K}$. Since a maximum magnitude magnetic field of $20 \mathrm{kOe}$ is strong enough to increase the Curie temperature above $285 \mathrm{~K}$, it follows that a simultaneous magnetocrystalline transformation results in a giant magnetostriction of about $-4500 \mathrm{ppm}$, which is due to the transition between the monoclinic and orthorhombic crystal structure, and ferromagnetic and paramagnetic structures. Compared to the strain in the thermal expansion in Fig.7-30, the magnetostriction in Fig.7-34 is smaller, which is due to the fact that when magnetic field change rate is faster than the transition rate so that before the transition is completely finished, the magnetic field shifts the transition temperature above $285 \mathrm{~K}$, when the transition stopped. 


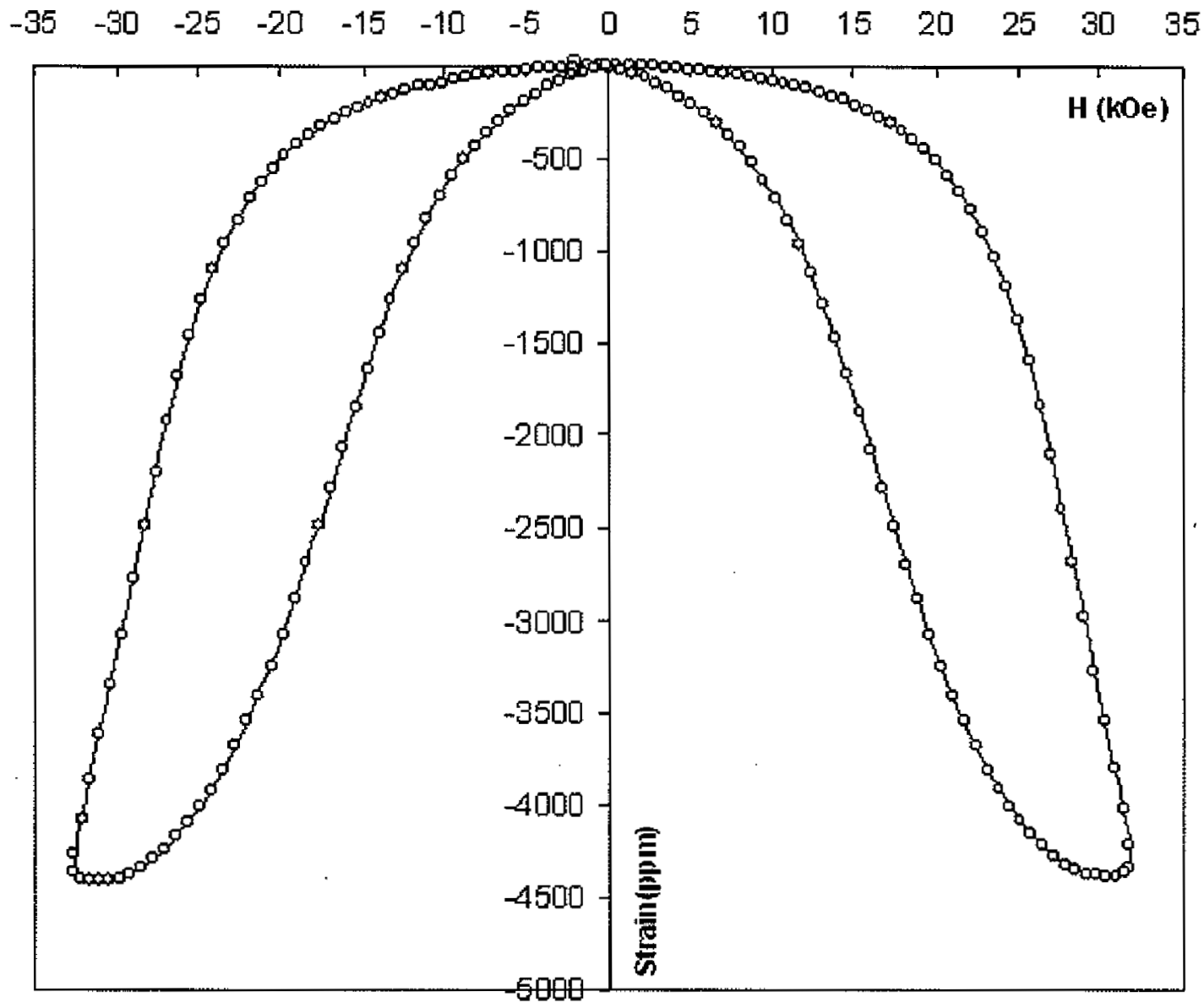

Fig.7-34 Magnetostriction measurement for polycrystal sample $\mathrm{Gd}_{5}\left(\mathrm{Si}_{2.09} \mathrm{Ge}_{1.91}\right)$ at $285 \mathrm{~K}$

As discussed before, different sample preparation methods can result in different grain size. According to the scanning electron microscopy (SEM) and transmission electron microscopy (TEM) studies by Chumbley et al [48], $\mathrm{Gd}_{5}\left(\mathrm{Si}_{\mathrm{x}} \mathrm{Ge}_{1-\mathrm{x}}\right)_{4}$ alloys were found to exhibit a "linear" feature in its microstructure $[47,48]$, as shown in Fig. 7-35 The linear features were found to exist in the samples which were in as prepared, polished, polished and etched conditions. Therefore, these linear structures were not due to the surface treatment and crystal growth [48]. 


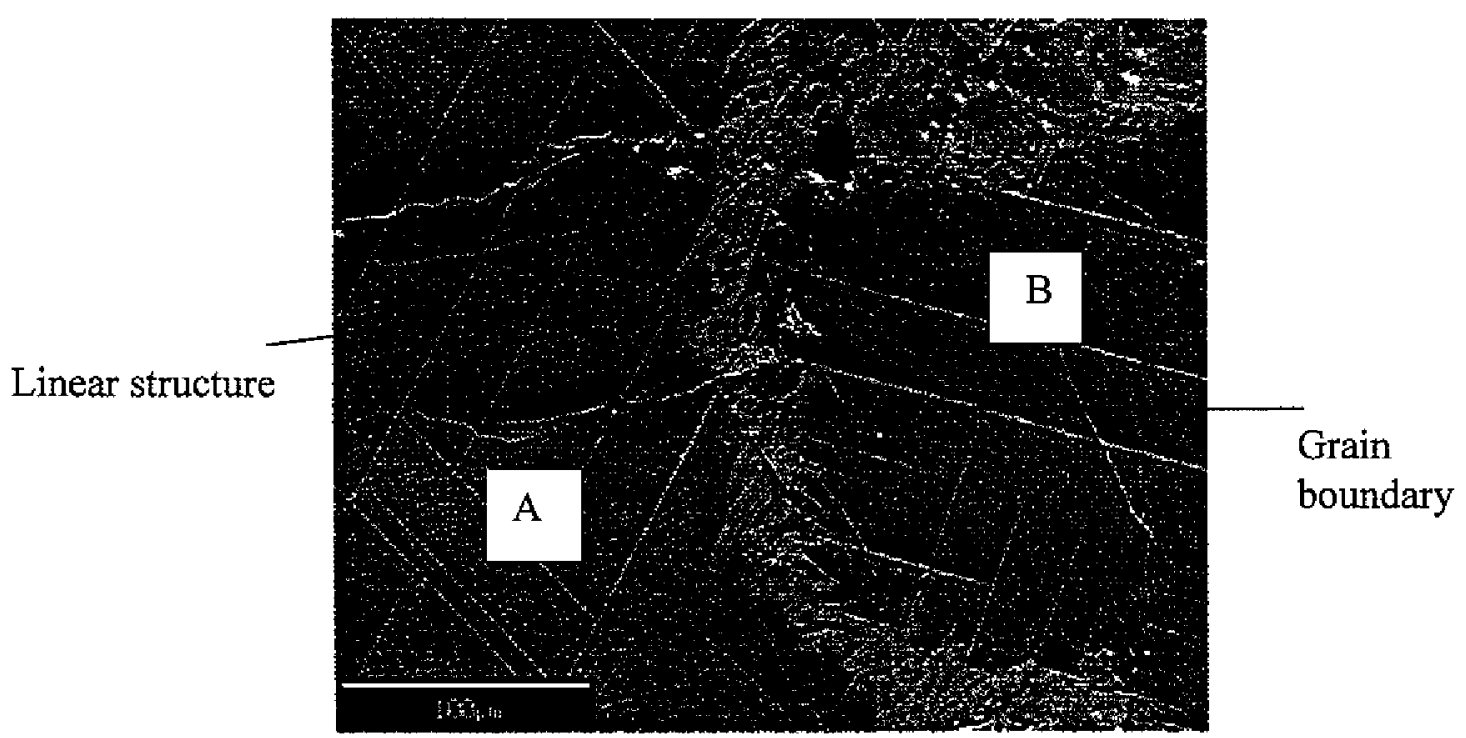

Fig.7-35 SEM picture of a polycrystalline sample $\mathrm{Gd}_{5}\left(\mathrm{Si}_{1.95} \mathrm{Gd}_{2.05}\right)$. Images taken by $\mathrm{J}$. Meyers [48].

The linear features are crystallographically oriented with respect to the crystal [48], and therefore they provide a means to distinguish different grains in a polycrystalline sample. In Fig. 7-35, the linear features in regions $\mathrm{A}$ and $\mathrm{B}$ show different orientations, indicating that they probably correspond to two grains. The grains appear to be in the order of hundreds of micron in size, although the image does not cover a large enough area for accurate measurements of the average grain size.

\section{Conclusions}

For $\mathrm{Gd}_{5}\left(\mathrm{Si}_{2,09} \mathrm{Ge}_{1.91}\right)$ samples prepared by the arc melting method, thermal expansion measurements have shown that a first order magneto-structural transformation occurs. Magnetic force microscopy has also been used to demonstrate the magnetic phase transformation process from paramagnetic to ferromagnetic upon cooling. It was found that 
the Curie temperature was lower and the thermally-induced strain higher, in the sample made from lower purity level Gd starting materials compared with the sample made from high purity Gd metal. These results indicate that the impurities (mainly $\mathrm{C}, \mathrm{O}, \mathrm{N}$ and $\mathrm{F}$ ) in the $\mathrm{Gd}$ starting material can significantly alter the transformation including the strain and Curie temperature of $\mathrm{Gd}_{5}\left(\mathrm{Si}_{\mathrm{x}} \mathrm{Ge}_{1-\mathrm{x}}\right)_{4}$ alloys.

For the sample prepared by induction melting method, thermal expansion measurements under $0,0.5,1.0,1.5,2.0$ Telsa magnetic fields were conducted. The results showed that the transformation is also first order, and has a temperature hysteresis about $2 \mathrm{~K}$. The transition temperature was found to be magnetic field dependent. On cooling, the change is about 6.2 $\mathrm{K} /$ Tesla. On heating, it is about $6.6 \mathrm{~K} /$ Tesla. The Curie temperature was higher when it was prepared by induction melting method than that prepared by arc melting method. Two different kinds of magnetostriction were found at $240 \mathrm{~K}, 285 \mathrm{~K}$. One was the usual fieldinduced magnetostriction which was about $-40 \mathrm{ppm}$ at $240 \mathrm{~K}$. The other one was a giant spontaneous magnetostriction at $285 \mathrm{~K}$, with a value about $-4500 \mathrm{ppm}$. 


\section{$7.3 \mathrm{Gd}_{5}\left(\mathrm{Si}_{\mathrm{x}} \mathrm{Ge}_{4-\mathrm{x}}\right)$ with $\mathrm{x}$ range of $0.575 \leq \mathrm{x} \leq 1$}

In this range of compositions, a single crystal sample $\mathrm{Gd}_{5}\left(\mathrm{Si}_{2.3} \mathrm{Ge}_{1.7}\right)$ and a polycrystalline sample $\mathrm{Gd}_{5}\left(\mathrm{Si}_{3} \mathrm{Ge}_{1}\right)$ were studied. VSM and Magnetic Force Microscopy were used to study the anisotropy of the single crystal sample. Thermal expansion and magnetostriction measurements were conducted to study the behaviors of phase transformations on both samples.

\subsubsection{Experimental Details}

\section{Single crystal $\mathrm{Gd}_{5}\left(\mathrm{Si}_{2.3} \mathrm{Ge}_{1.7}\right)$}

The single crystal preparation method is the same as the single crystal samples discussed before.

Thermal expansion measurements were conducted at two temperature ranges: (1) between room temperature and $150 \mathrm{~K}$; (2) between $296 \mathrm{~K}$ and $340 \mathrm{~K}$. The linear thermal expansion was measured during both cooling and heating. Measurements were made either when the strain changed by $4 \mathrm{ppm}$ or the temperature changed by $1 \mathrm{~K}$. The transition temperatures were determined by finding the maximum point in the derivative of strain vs. temperature. For the magnetostriction measurement, the magnetic field was applied along the a, b, c-axis respectively, and the field was cycled between $-23 \mathrm{kOe}$ to $23 \mathrm{kOe}$ at room temperature. The isothermal magnetization process was conducted along $a, b, c$ axis by using the VSM at room temperature. The sample shape for the VSM measurement was cubic with weight of $0.0255 \mathrm{~g}$. The magnetization vs. temperature measurement was conducted between room temperature and $350 \mathrm{~K}$. Before the MFM images were taken on the $a, b$ and $c$ plane, the sample was magnetized under a field of 2 Telsa. 


\subsubsection{Results and discussions}

(a) Magnetization vs. Temperature

Magnetization vs. temperature measurement is shown in Fig.7- 35. The $1 / \chi$ vs. $T$ relation derived from this showed that it follows the Curie-Weiss law, suggesting that the high temperature phase is paramagnetic above $307 \mathrm{~K}$. On heating, it transits from the lower temperature ferromagnetic phase into the paramagnetic phase. On cooling, it transits from the paramagnetic state into the ferromagnetic state. The Curie temperature was found from extrapolation of the $1 / \chi-\mathrm{T}$ curve to be $307 \mathrm{~K}$ as shown in Fig. 7-36. According to ref [14] with an $\mathrm{x}$ value of 0.575 , the crystal structure is the $\mathrm{Gd}_{5} \mathrm{Si}_{4}$-type orthorhombic structure with space group Pnma. During the transformation process, there was no observable structure change. Choe [16] et al. has pointed out that the $\mathrm{Si}(\mathrm{Ge})-\mathrm{Si}(\mathrm{Ge})$ bonds between the adjacent building layers, which are responsible for the superexchange interaction between the $\mathrm{Gd}$ atoms in the adjacent slab, are not broken. The temperature "hysteresis" found in the Fig.735 is most likely attributable to experimental error. Therefore, it is a second order transformation for the Si-rich region of $\mathrm{Gd}_{5}\left(\mathrm{Si}_{x} \mathrm{Ge}_{1-\mathrm{x}}\right)_{4}$. 


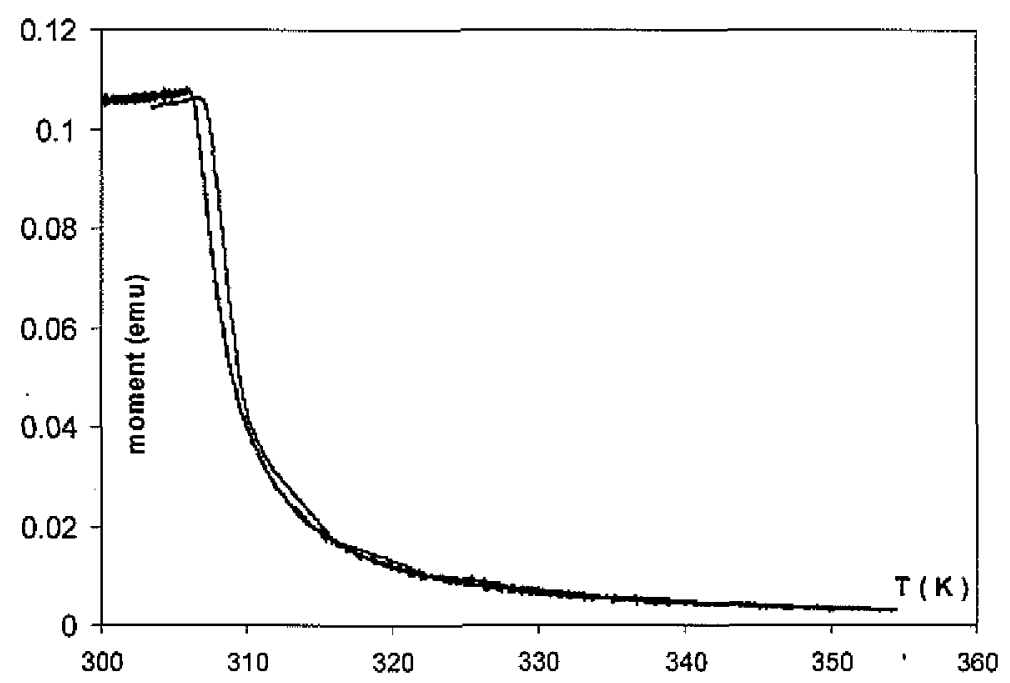

Fig.7- 35 The $\mathrm{M}$ vs $\mathrm{T}$ for $\mathrm{Gd}_{5}\left(\mathrm{Si}_{2.3} \mathrm{Ge}_{1.7}\right)$ single crystal

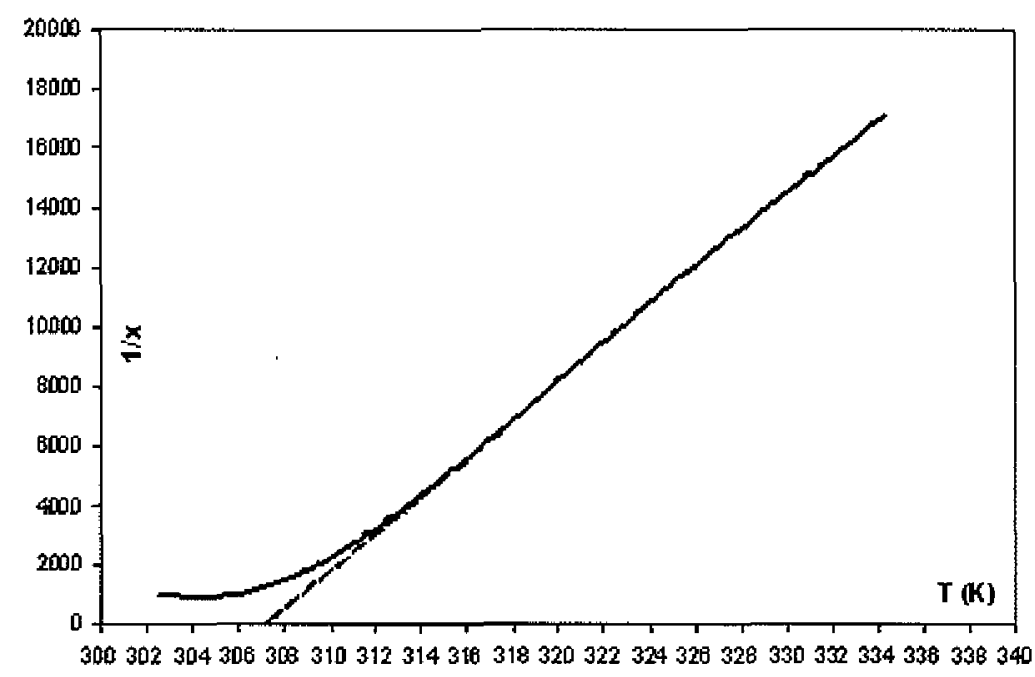

Fig.7- 36 The $1 / \chi$ vs. T relationship of singe crystal $\mathrm{Gd}_{5}\left(\mathrm{Si}_{2.3} \mathrm{Ge}_{1.7}\right)$

\section{(b) VSM measurement}

The $\mathrm{M}$ vs. $\mathrm{H}$ relationship is shown in Fig.7-37, which was measured at room temperature along $\mathrm{a}, \mathrm{b}$ and $\mathrm{c}$ axes. It is clear from this that it is a soft ferromagnetic material with coercivity of $7 \mathrm{Oe}$. The saturation magnetization moment was found to be $87 \mathrm{emu} / \mathrm{g}(0.64 \times$ 
$10^{6} \mathrm{~A} / \mathrm{m}$ ). The $\mathrm{M}-\mathrm{H}$ hysteresis loops for $\mathrm{a}, \mathrm{b}$ and $\mathrm{c}$ axis were shown in Fig. 7-37, the $\mathrm{c}$ axis was found to be the easy axis, b axis was found to be the hard axis, which is very different from the $\mathrm{Gd}_{5}\left(\mathrm{Si}_{2} \mathrm{Ge}_{2}\right)$ sample, which showed that the $b$ axis was found to be the easy axis. The VSM measurement also support the conclusion drawn previously: at room temperature, it is in ferromagnetic state.

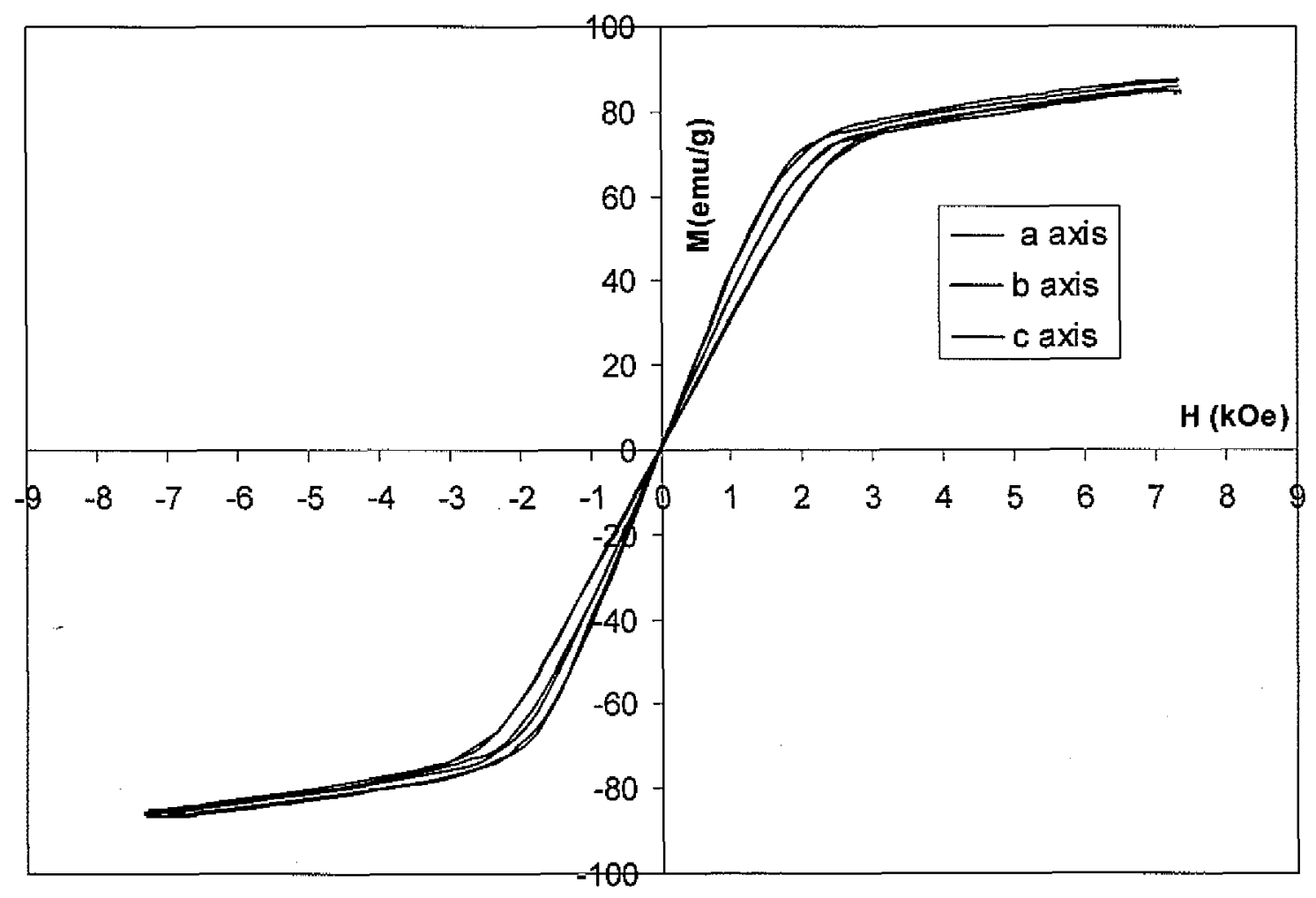

Fig.7-37 The hysteresis loops along the $\mathrm{a}, \mathrm{b}$ and $\mathrm{c}$ axis at room temperature.

\section{(c) Thermal expansion measurements}

(1) Along the $\boldsymbol{a}$ axis

Before the measurement, the sample was in the demagnetized state, no magnetostriction having been done before. Based on the previous results, there should be no first or second order transitions below room temperature. Thermal expansion measurements were shown in 
Figs.7-38, 39, 40. Above room temperature, there was an abnormal behavior found at $T=$ $305 \mathrm{~K}$ in Fig. 7-40. Tc is consistent with the result found in Fig. 7-36. Therefore, it is believed that this is due to the second order transformation between the ferromagnetic and the paramagnetic phases. Below room temperature, thermal expansion measurement results are shown in Fig. 7-38 and 7-39.

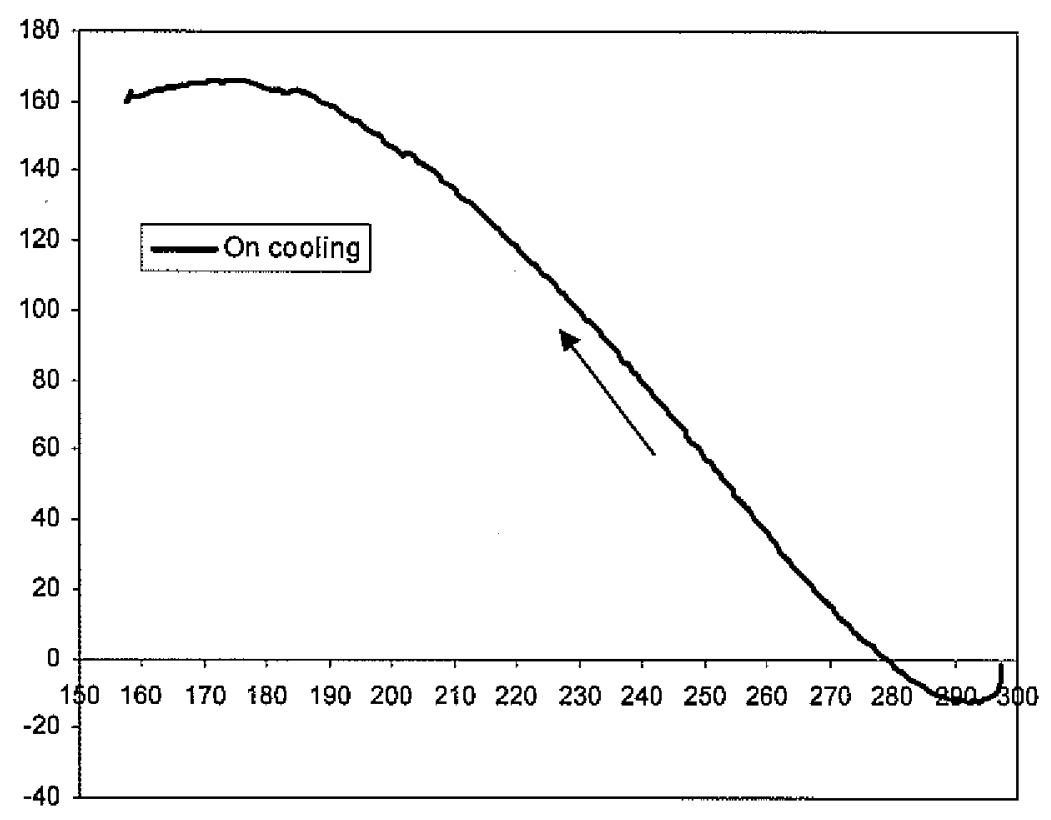

Fig.7-38 Thermal expansion for $\mathrm{Gd}_{5}\left(\mathrm{Si}_{2.3} \mathrm{Ge}_{1.7}\right)$ along a axis on cooling from room temperature

After correcting the measurement error caused by the voltage shift, the heating curve is shown in Fig.7-39. 


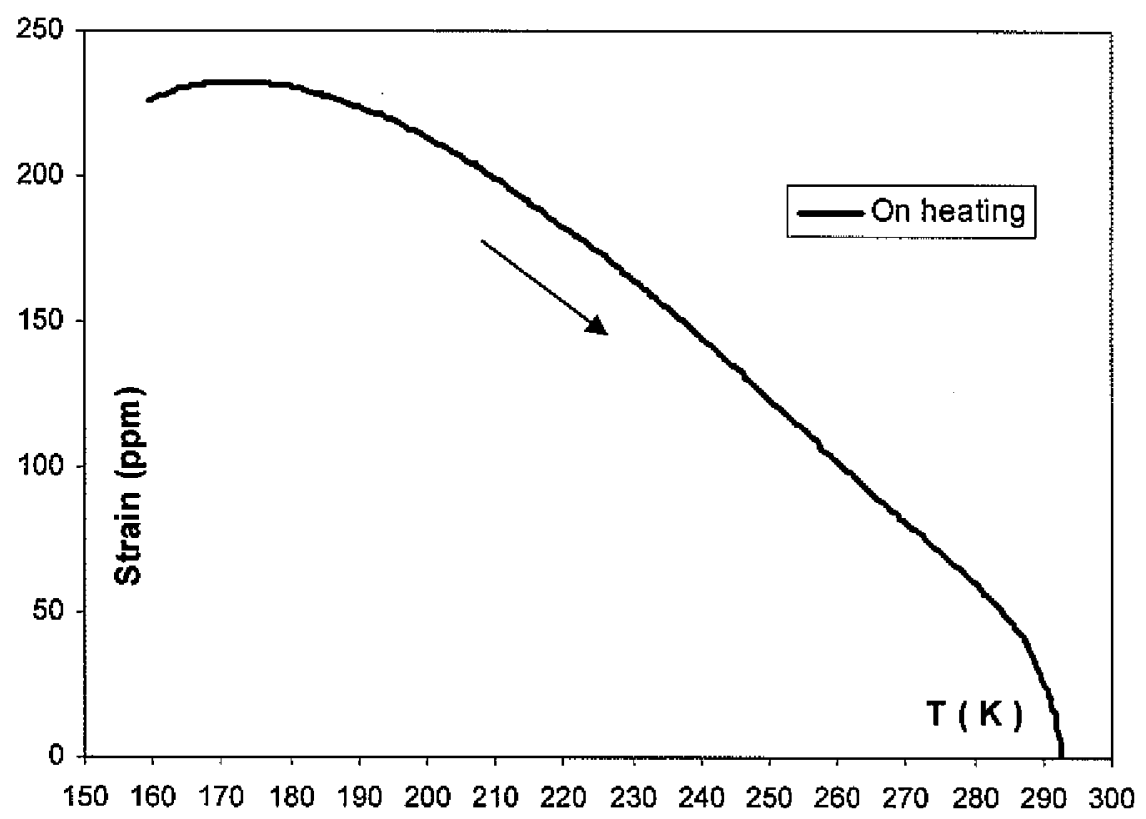

Fig. 7-39 Thermal expansion for $\mathrm{Gd}_{5}\left(\mathrm{Si}_{2,3} \mathrm{Ge}_{1.7}\right)$ along a axis on heating to room temperature

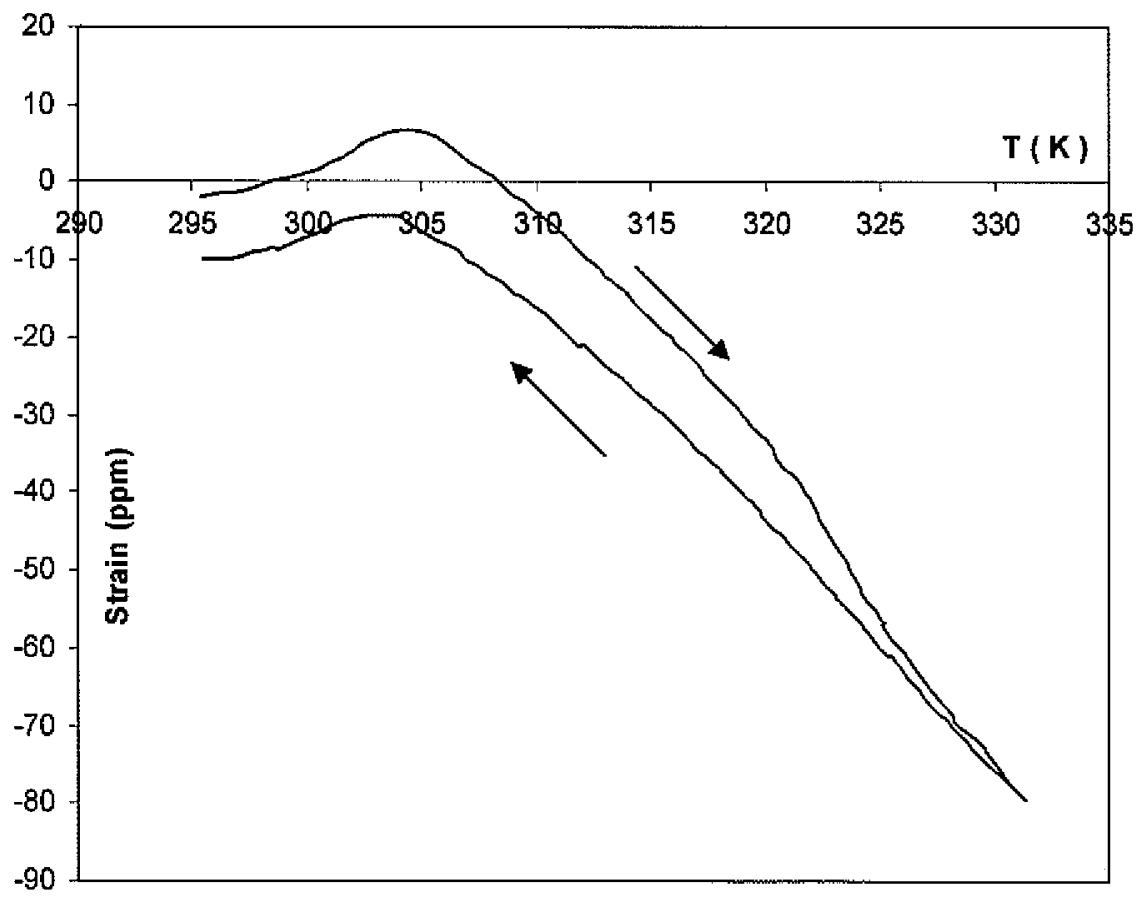

Fig. 7-40 Thermal expansion for $\mathrm{Gd}_{5}\left(\mathrm{Si}_{2.3} \mathrm{Ge}_{1.7}\right)$ along the a axis on heating from room temperature 
(2) Along $\mathbf{b}$ axis

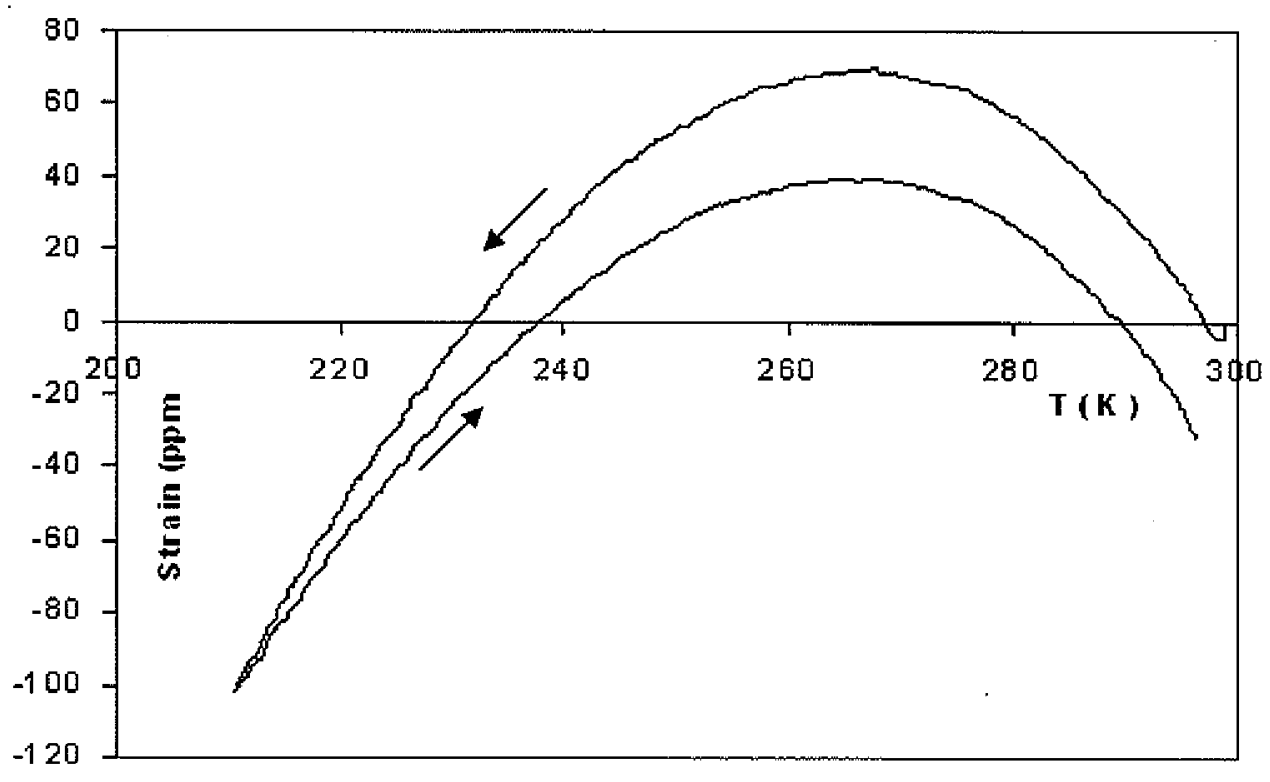

Fig.7- 41 Thermal expansion for $\mathrm{Gd}_{5}\left(\mathrm{Si}_{2.3} \mathrm{Ge}_{1.7}\right)$ along $\mathrm{b}$ axis between $210 \mathrm{~K} \sim 300 \mathrm{~K}$

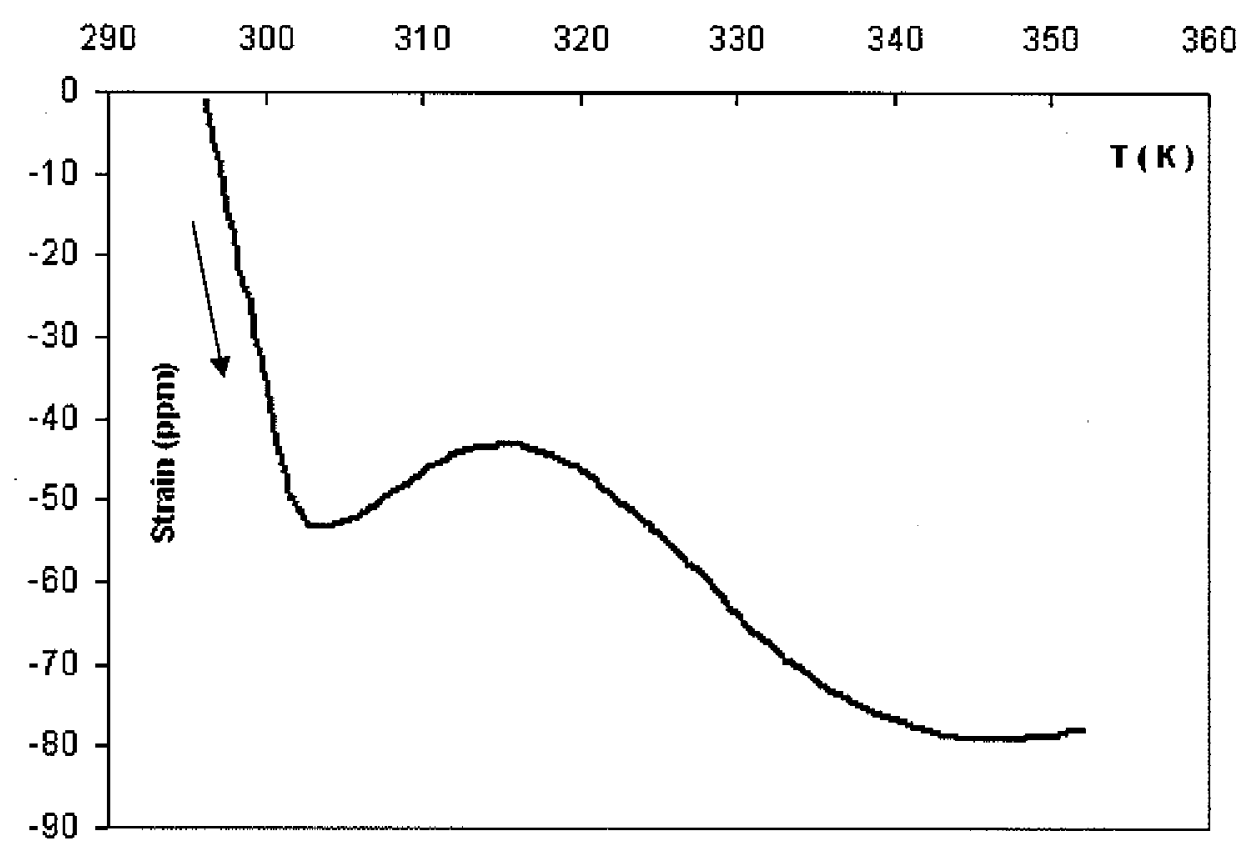

Fig.7-42 Thermal expansion for $\mathrm{Gd}_{5}\left(\mathrm{Si}_{2.3} \mathrm{Ge}_{1.7}\right)$ along $\mathrm{b}$ axis between $300 \mathrm{~K} \sim 350 \mathrm{~K}$, the peak in blue circle is due to the second order transition. 
Fig. 7-41 shows the thermal expansion measurement below room temperature. Fig.7-42 shows thermal expansion measurement above room temperature. Combined with the $\mathrm{Tc}$ found by the $M$ vs. $T$ measurement, the anomalous behavior found at $T=305 \mathrm{~K}$ in Fig. $7-42$ is due to the second order transition. The peak between $310 \mathrm{~K}$ and $320 \mathrm{~K}$ was due to an unknown reason, a possible explanation is that disorder of spins in the short range caused by the increasing energy.

(3) Along the $c$ axis

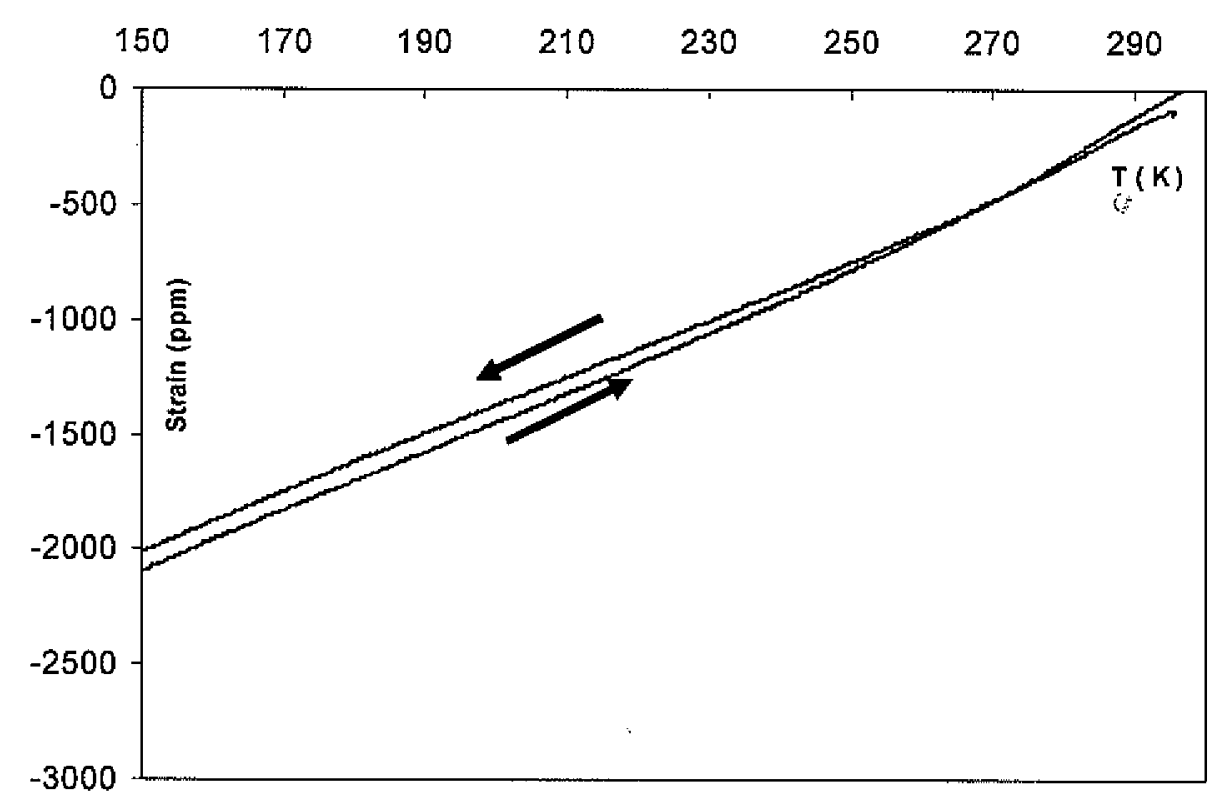

Fig.7-43 Thermal expansion for $\mathrm{Gd}_{5}\left(\mathrm{Si}_{2,3} \mathrm{Ge}_{1,7}\right)$ along $\mathrm{c}$ axis between $150 \mathrm{~K} \sim 300 \mathrm{~K}$ 


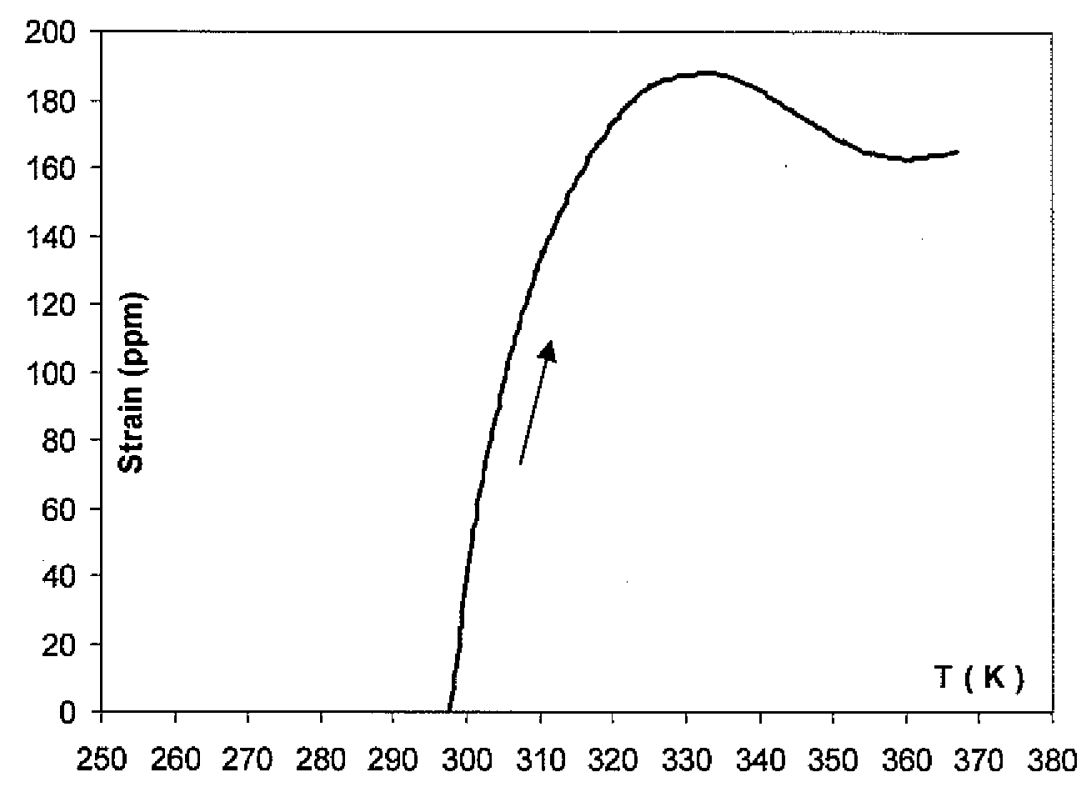

Fig.7-44 Thermal expansion for $\mathrm{Gd}_{5}\left(\mathrm{Si}_{2.3} \mathrm{Ge}_{1.7}\right)$ along $\mathrm{c}$ axis between $300 \mathrm{~K} \sim 350 \mathrm{~K}$

It is very interesting to note that the thermal expansion measurement along the $\mathrm{c}$ axis shown in Fig. 7-43, no anomalous behavior like long a, b axes was found. Above room temperature, there was a peak found as shown in Fig. 7-44, but no at about $\mathrm{T}=305 \mathrm{~K}$. 
(c) Magnetostriction measurements

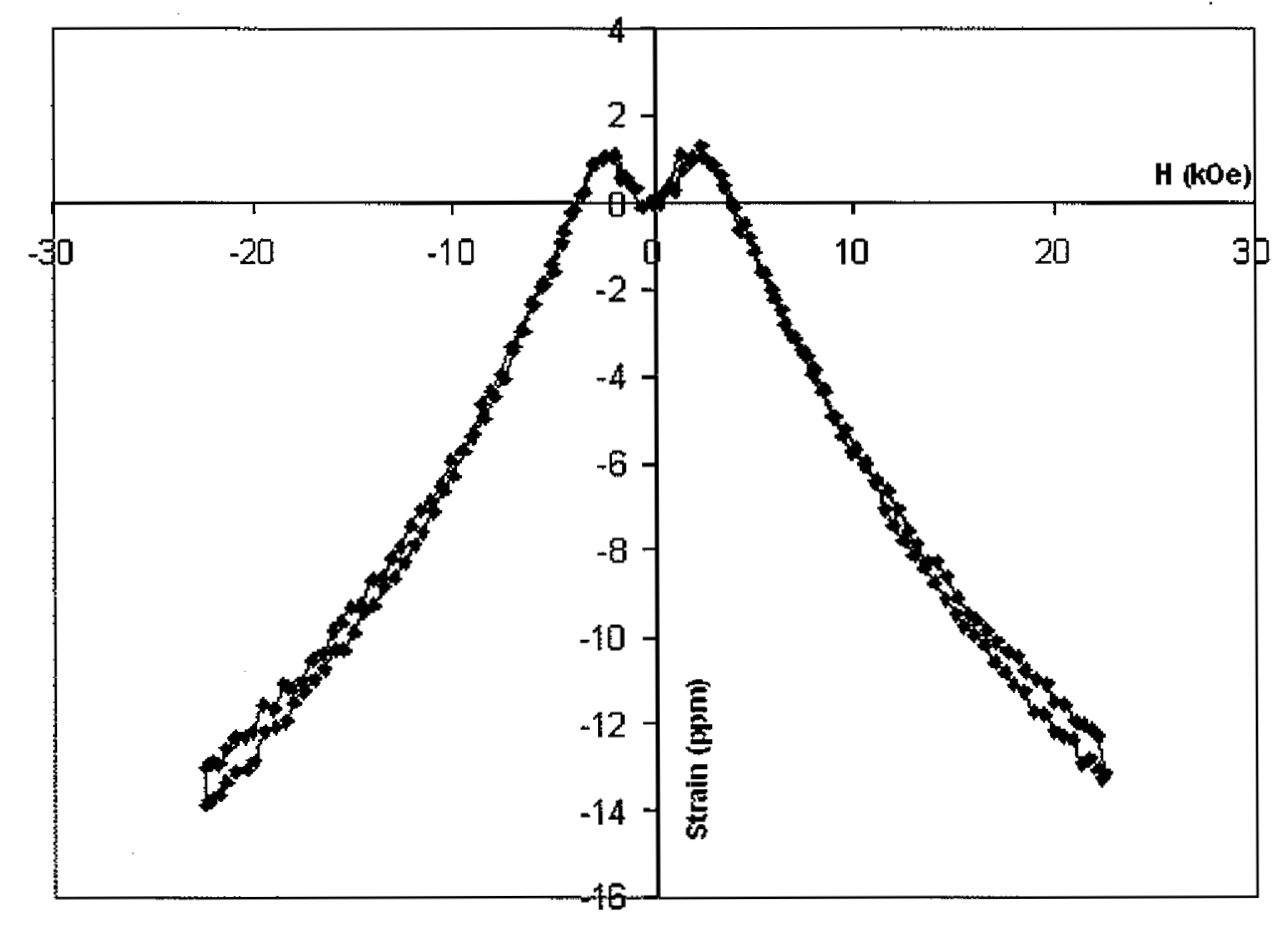

Fig.7-45 Magnetostriction measurement at room temperature for $\mathrm{Gd}_{5}\left(\mathrm{Si}_{2.3} \mathrm{Ge}_{1.7}\right)$ along a axis

This measurement of magnetostriction along the a axis is shown in Fig.7-45, which shows forced magnetostriction. The magnetostriction along $\mathrm{b}$ and $\mathrm{c}$ axes is shown in Fig.7-46, 47. The sign of magnetostriction along $a, b$ and $c$ axes is negative, positive and negative respectively. All of them were very small, compared to the giant magnetostriction found at other values of $\mathrm{x}$, where there exists a simultaneous magneto-structural transformation. These observed phenomena show a strong anisotropy of magnetostriction. The maximum magnetostriction values along a, $b, c$ axes are -14 ppm, 100 ppm, -135 ppm respectively. 


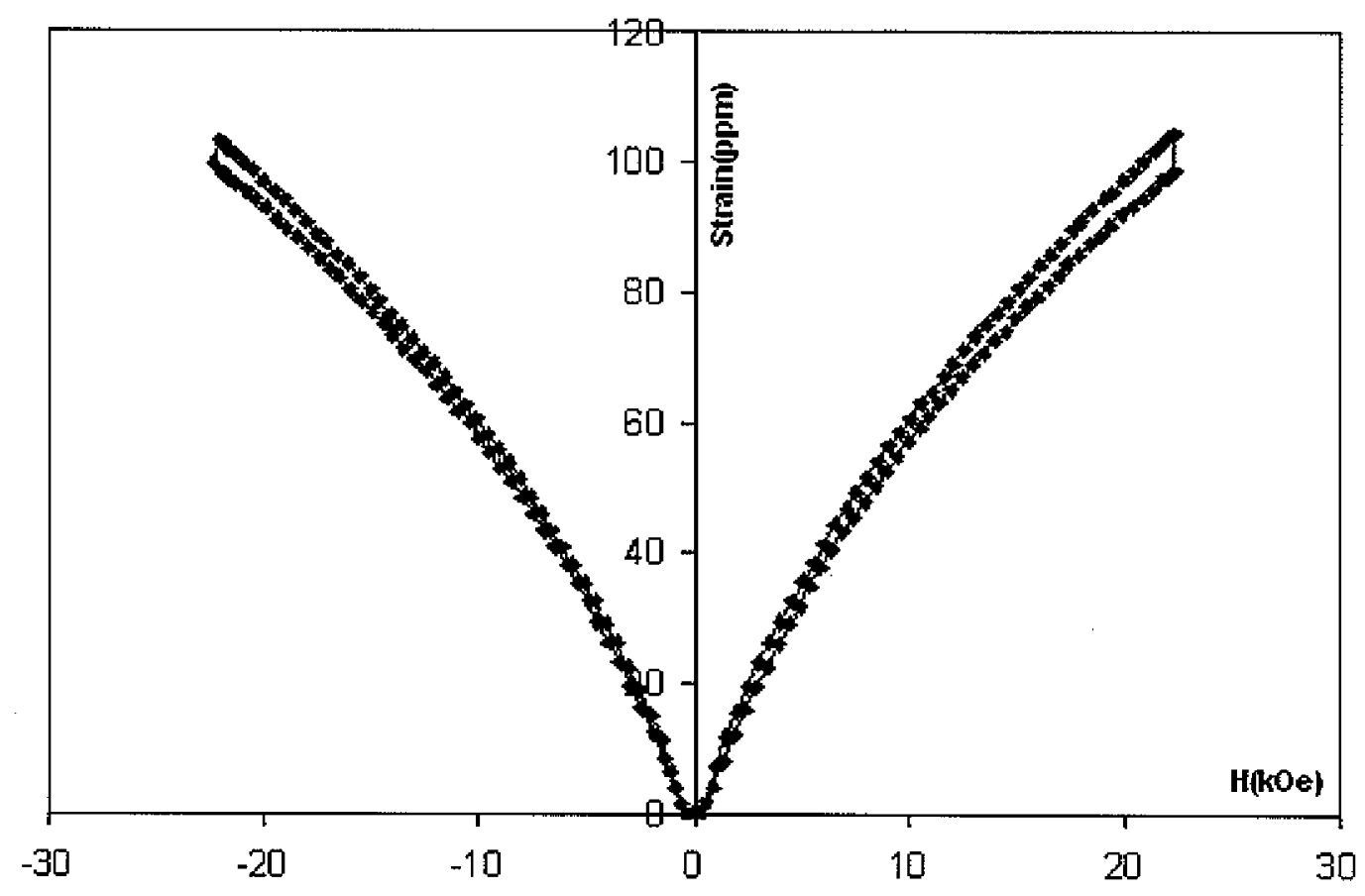

Fig.7-46 Magnetostriction measurement for $\mathrm{Gd}_{5}\left(\mathrm{Si}_{2.3} \mathrm{Ge}_{1.7}\right)$ along $b$ axis at room temperature

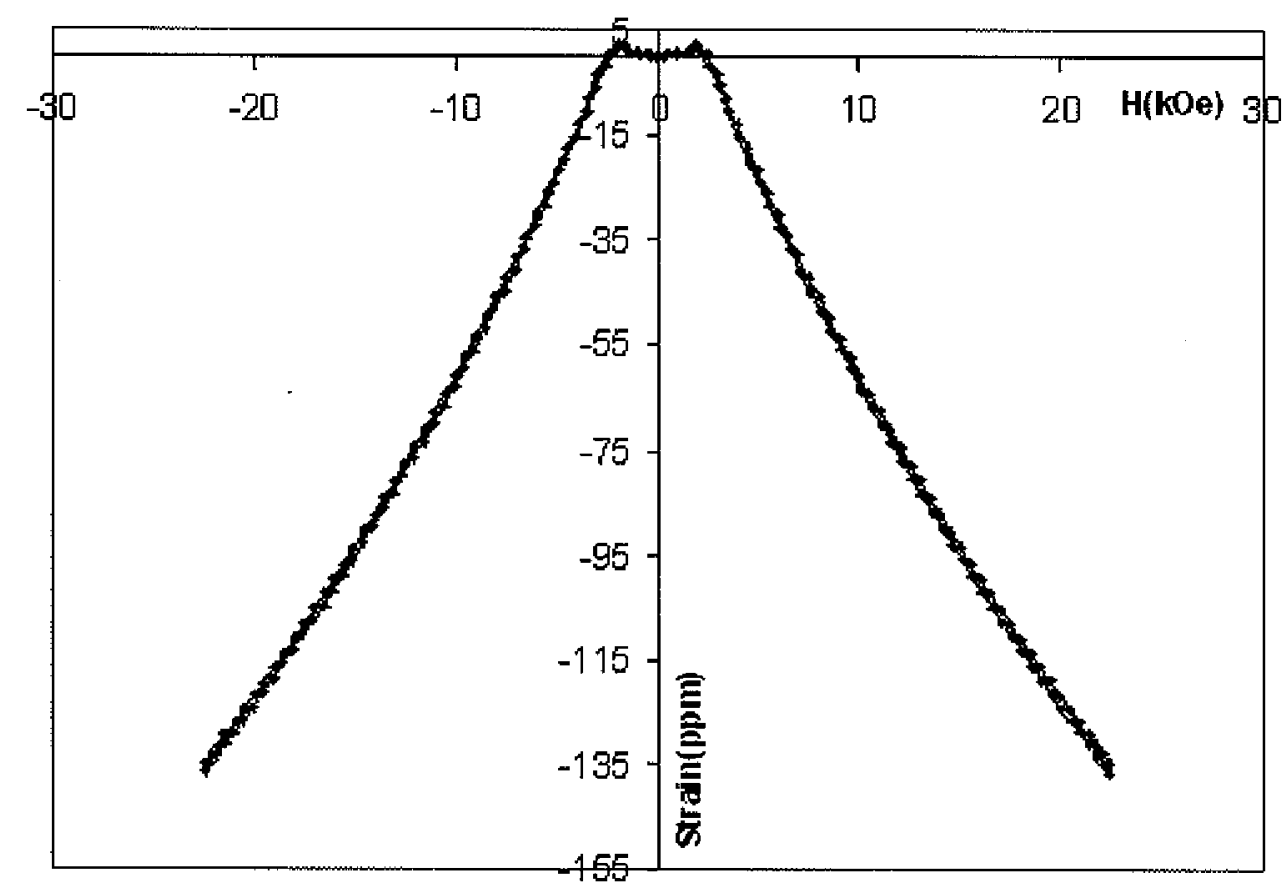

Fig.7-47 Magnetostriction measurement for $\mathrm{Gd}_{5}\left(\mathrm{Si}_{2.3} \mathrm{Ge}_{1.7}\right)$ along $\mathrm{c}$ axis at room temperature 
(d) MFM images taken on $\mathrm{a}, \mathrm{b}$ and $\mathrm{c}$ planes

MFM images taken on a, b, c planes were shown in Fig.7-48, 49 and 50. On the a, b plane, the images show that the magnetization in each domain in the selected area are oriented at random, resulting in a homogenous contrast distribution, some dark areas are caused by the surface topography, not the domain structures. However, on the c plane, we observed well defined stripe domain structures, which are formed by the 180 degree domain structures. The magnetizations in adjacent domains are aligned antiparallel. Therefore, we observed a strong anisotropy in domain structure between the $\mathrm{a}, \mathrm{b}$ and $\mathrm{c}$ planes.

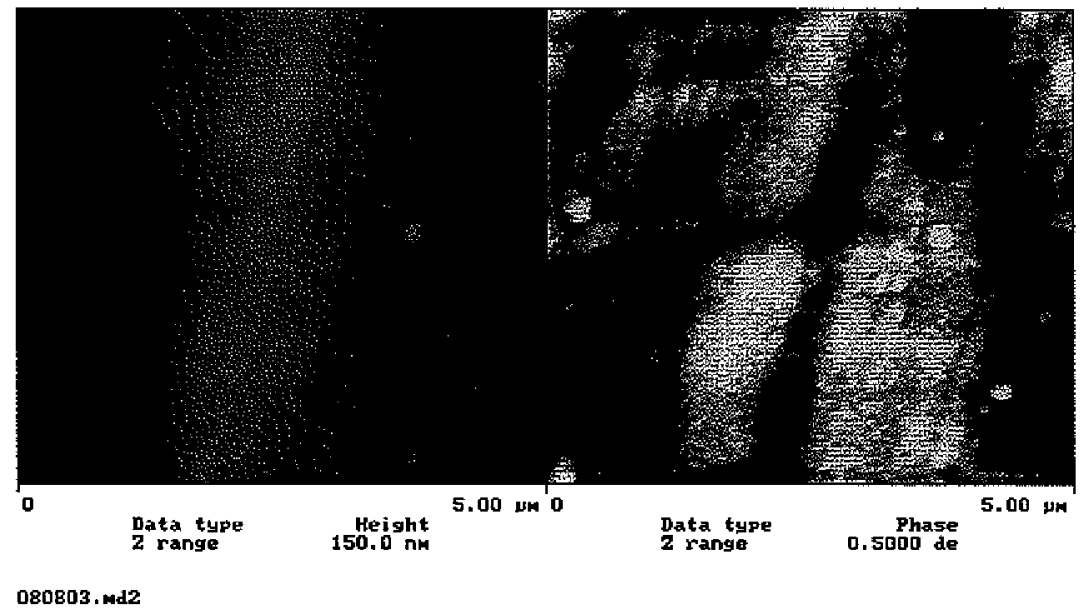

Fig.7-48 MFM image taken on the a plane. Left hand image is topographic, right hand image is magnetic force. 


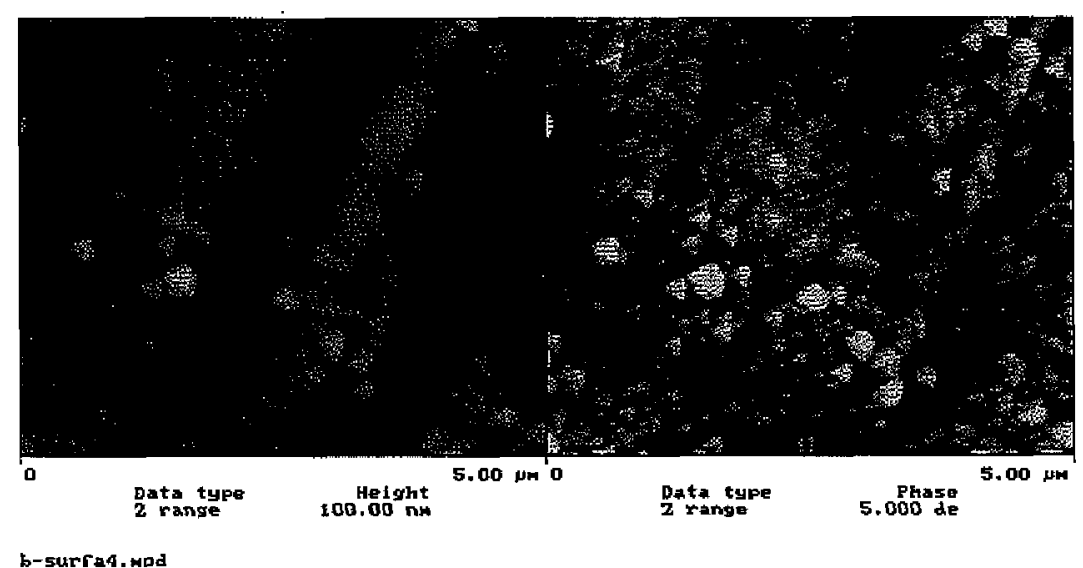

Fig.7-49 MFM image taken on the $b$ plane. Left hand image is topographic, right hand image is magnetic force.

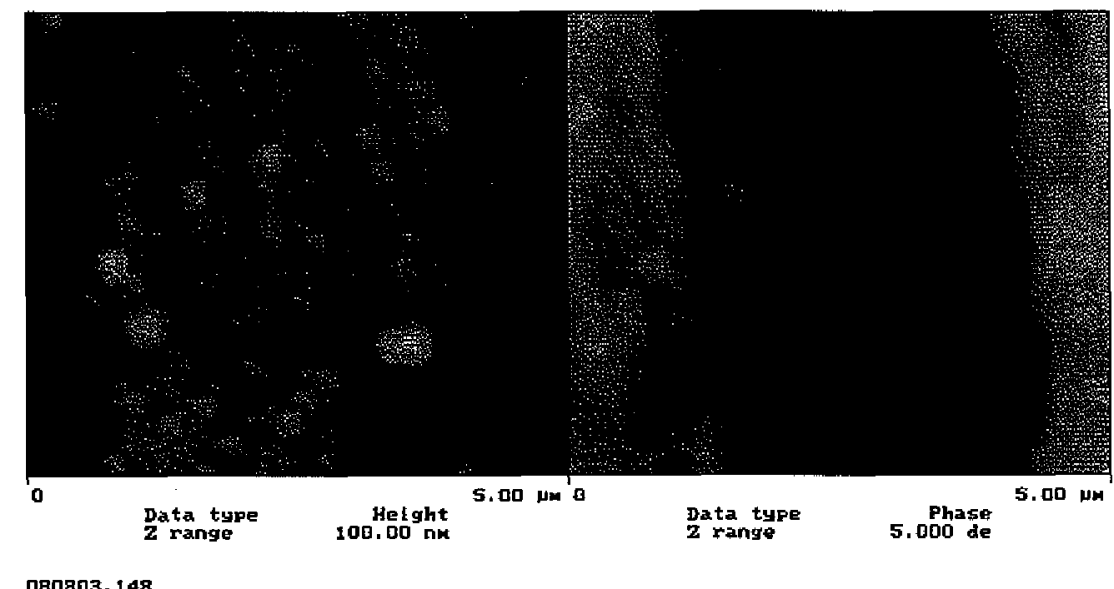

Fig.7-50 MFM image taken on the $c$ plane. Left hand image is topographic, right hand image is magnetic force.

\section{Conclusions}

The $M$ vs. $T$ measurement shows that for $\mathrm{Gd}_{5}\left(\mathrm{Si}_{2,3} \mathrm{Ge}_{1.7}\right)$ there exists a second order transformation at $\mathrm{T}=307 \mathrm{~K}$ : below $307 \mathrm{~K}$, it is in a ferromagnetic state; above $307 \mathrm{~K}$, it is in a paramagnetic state. The $\mathrm{M}$ vs. $\mathrm{H}$ measurements showed that it is anisotropic along $\mathrm{a}, \mathrm{b}$ and c axes and it is a soft magnetic material with coercivity of 7 Oe. Thermal expansion measurement shows a strong anisotropy not only in the measurement above room 
temperature, but also in the below temperature measurement. Magnetostriction measurements at room temperature along $a, b, c$ axes showed a small strain value, but a strong anisotropic behavior. MFM images taken on $a, b$ and $c$ plane demonstrated that the domain structures are also strongly anisotropic.

\subsubsection{Polycrystalline sample of $\mathrm{Gd}_{5}\left(\mathrm{Si}_{3} \mathrm{Ge}_{1}\right)$}

\section{Experimental Details}

The polycrystal sample was prepared by arc-melting method with AL Gd. Detailed preparation method can be found in the previous section in this chapter. The sample was first heated from room temperature to $326 \mathrm{~K}$, and then cooled down to the $220 \mathrm{~K}$ under zero magnetic field. Magnetostriction measurement was conducted at room temperature with magnetic field changing from $0 \mathrm{kOe}$ to $28 \mathrm{kOe}$. VSM measurement was conducted at room temperature by using the IDEAS-VSM Model 4500, Lake Shore Cryotronics, Inc., Magnetization versus temperature measurement was conducted in VSM between $295 \mathrm{~K}$ and $385 \mathrm{~K}$.

\section{Results and discussions}

According to the ref [14], for $\mathrm{Gd}_{5}\left(\mathrm{Si}_{3} \mathrm{Ge}_{1}\right)$, the crystallographic structure is orthorhombic (space group Pnma). The crystallographic data for it are: a axis $(7.4972 \AA)$, b axis $(14.765$ $\AA$ ), and c axis (7.7709 $\AA$ ), its unit cell volume is about $860.2 \AA^{3}$. Choe et al. [16], suggested that $\mathrm{Gd}_{5}\left(\mathrm{Si}_{\mathrm{x}} \mathrm{Ge}_{1-\mathrm{x}}\right)_{4}$ in the range of $0.5<\mathrm{x}<1$, there only exists one magnetic phase transition: paramagnetic $\leftrightarrow$ ferromagnetic. No crystal structure transformation is coupled with it, as was observed in the other ranges of $\mathrm{x}$. Our thermal expansion measurement between $220 \mathrm{~K}$ and $326 \mathrm{~K}$ under zero magnetic field are shown in Fig.7-51. This thermal expansion curve gives 
no evidence of a structural transition, but shows only a magnetic transition, Such behavior is unlike that found for other values of $\mathrm{x}$ within region II, which have been discussed before. The Curie point was found to be about $319 \mathrm{~K}$ from the plot of $1 / \chi$ vs. temperature in Fig.7-52 (b). The $M$ vs $T$ curve in Fig.7-52 (a) and (b) are a typical curve which follows the CurieWeiss mean-field theory, and there is no temperature hysteresis during heating and cooling. This also confirms that it is a second order transition.

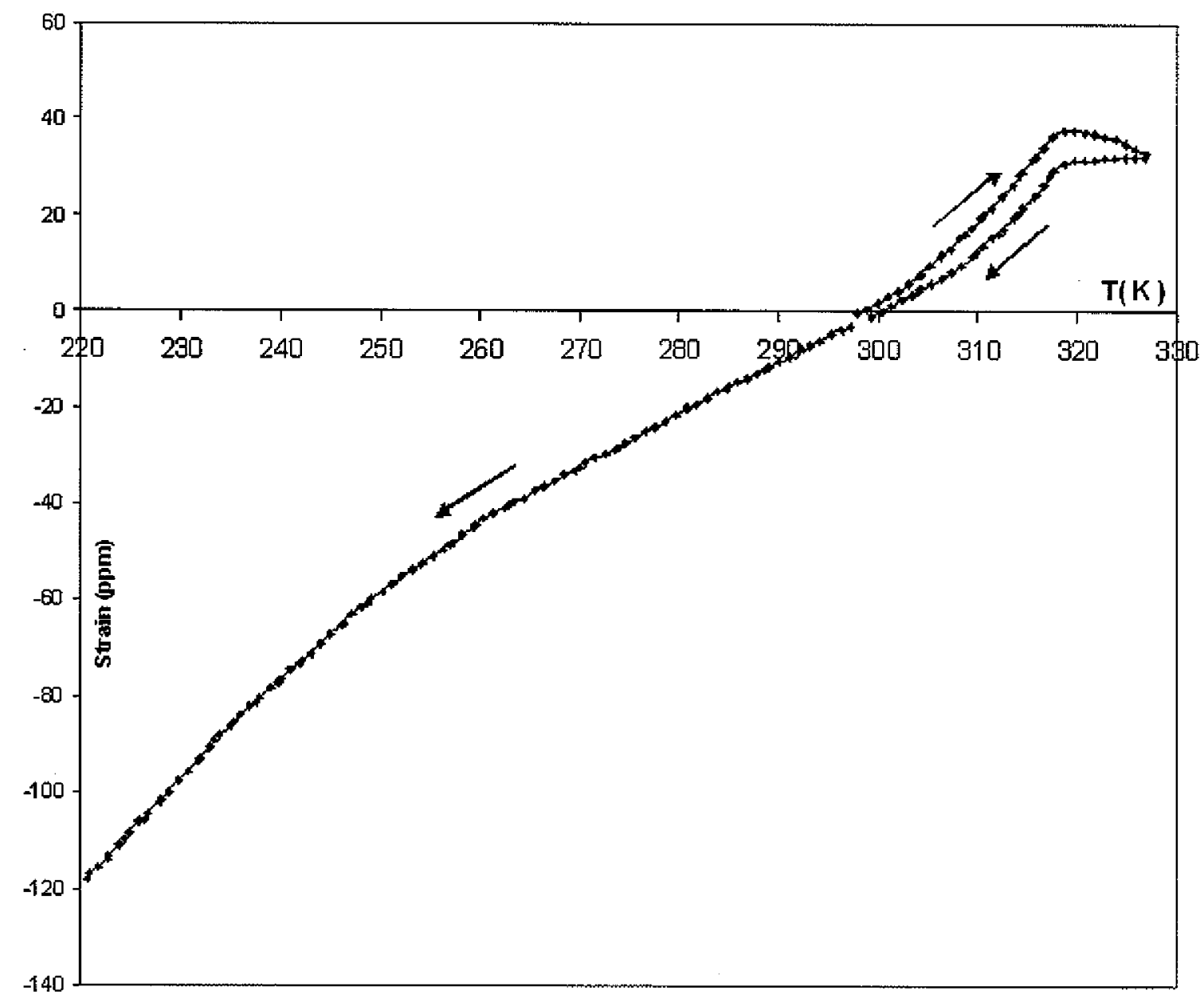

Fig.7-51 Thermal expansion for polycrystal sample $\mathrm{Gd}_{5}\left(\mathrm{Si}_{3} \mathrm{Ge}_{1}\right)$ 


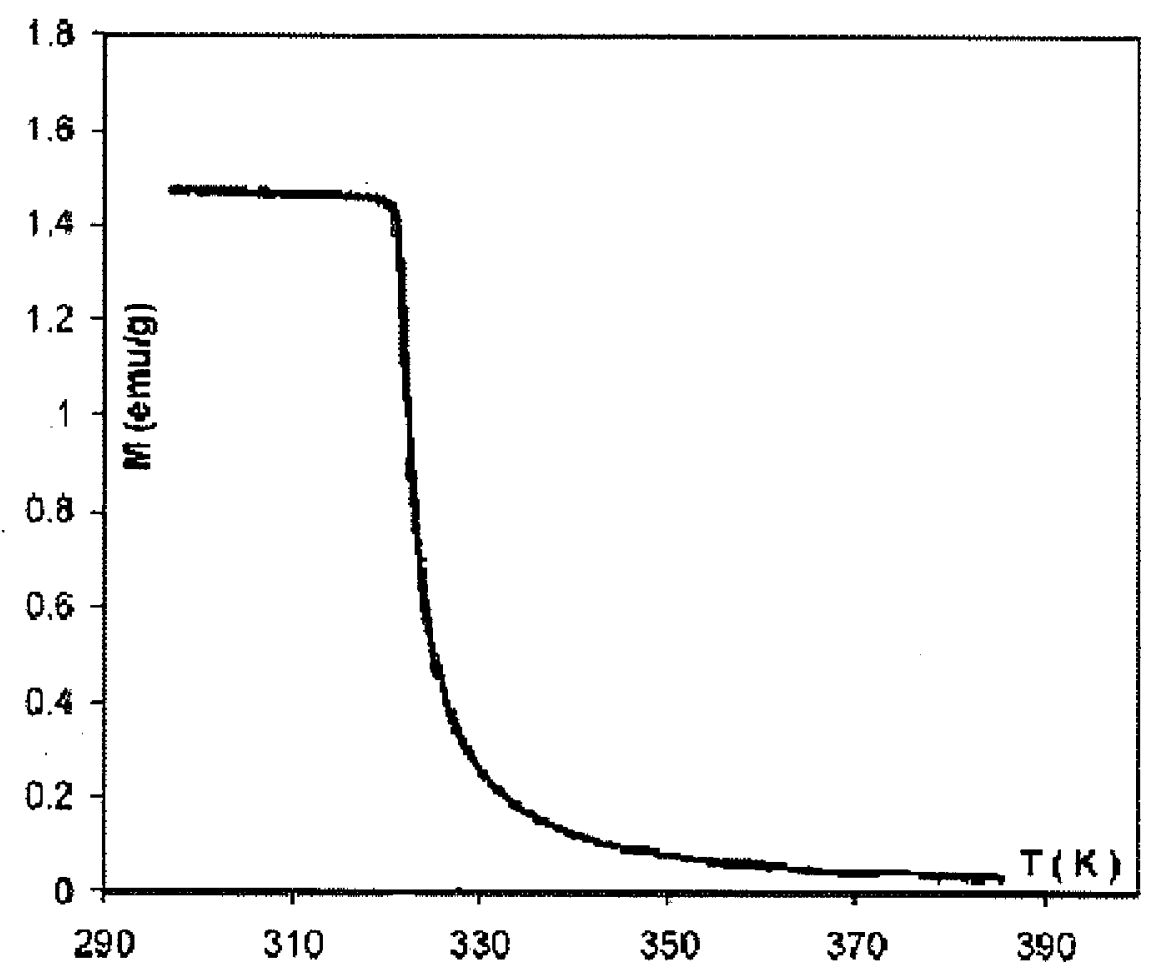

Fig.7-52 (a) M vs $\mathrm{T}$ measurement for $\mathrm{Gd}_{5}\left(\mathrm{Si}_{3} \mathrm{Ge}_{1}\right)$

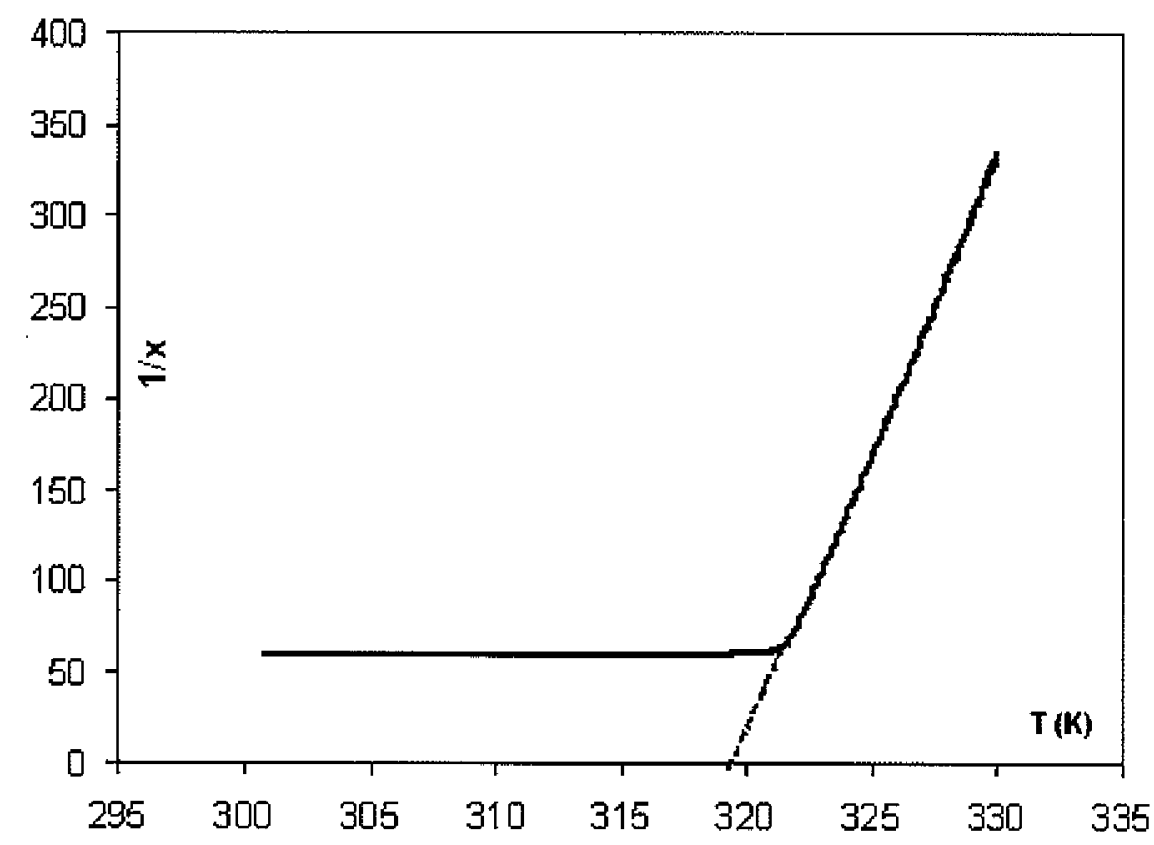

Fig. $7-52$ (b) $1 / \chi$ vs $\mathrm{T}$ relation for $\mathrm{Gd}_{5}\left(\mathrm{Si}_{3} \mathrm{Ge}_{1}\right), \mathrm{Tc}=319 \mathrm{~K}$ 


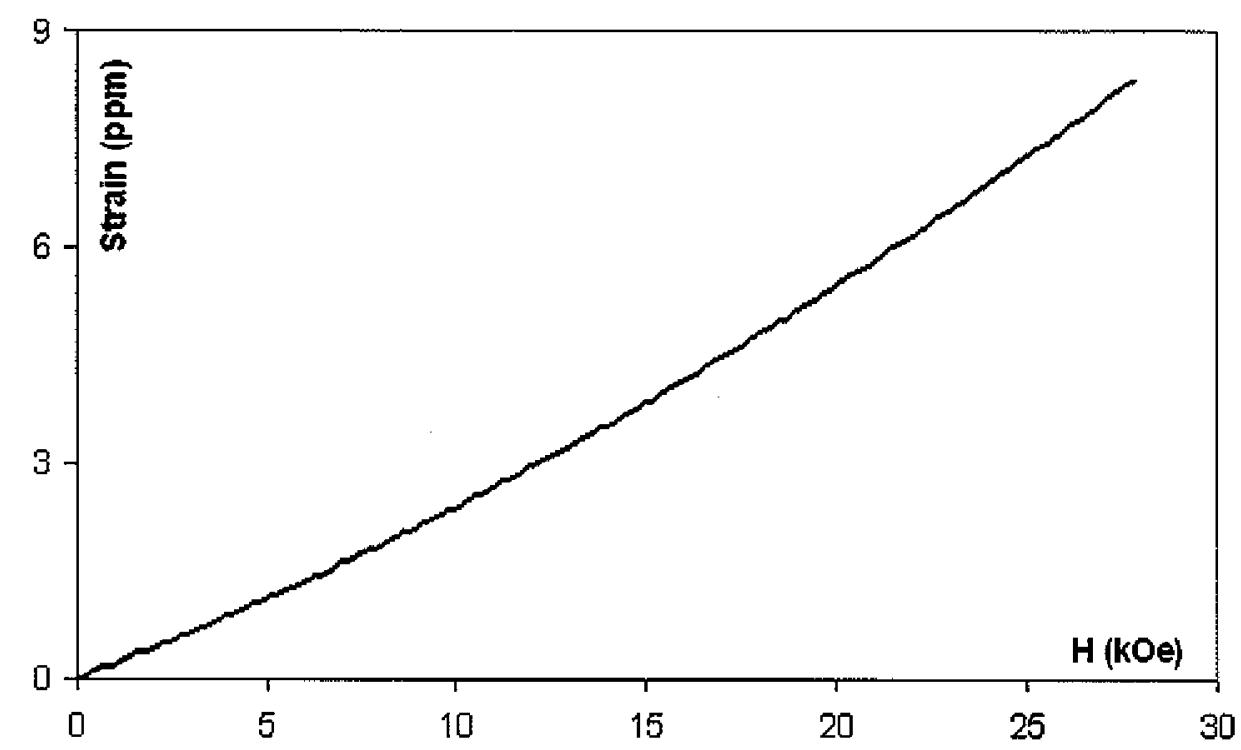

Fig.7-53 Magnetostriction measurement at room temperature

In Fig.7-53, magnetostriction results show a very small value, only about 8ppm under 28 kOe. In ref $[36,37]$, a polycrystal sample $\mathrm{Gd}_{5}\left(\mathrm{Si}_{1.8} \mathrm{Ge}_{2.2}\right)$ exhibited a giant magnetostriction above the Curie temperature, this phenomenon was also found in single crystal $\mathrm{Gd}_{5}\left(\mathrm{Si}_{2} \mathrm{Ge}_{2}\right)$ along c axis [41]. It is believed that these two magnetostriction values are due to different mechanism. For $\mathrm{Gd}_{5}\left(\mathrm{Si}_{3} \mathrm{Ge}_{1}\right)$ in these case, where there is no structural transformation at the Curie temperature, the magnetostriction is due to the non- $180^{\circ}$ domain wall movement. However, for $\mathrm{Gd}_{5}\left(\mathrm{Si}_{1.8} \mathrm{Ge}_{2.2}\right)$ and $\mathrm{Gd}_{5}\left(\mathrm{Si}_{2} \mathrm{Ge}_{2}\right)$, these are first order magnetocrystalline transitions, where a structural transition from the monoclinic to the orthorhombic, and a magnetic transition from the paramagnetic to ferromagnetic occurs together. Therefore giant magnetostriction was observed. As we know the physical origin of "normal" field-induced magnetostriction is due the spin-orbit coupling. However this material is very different from pure Gd, because in Gd the orbit angular momentum is zero and the spin-orbit coupling is zero. 


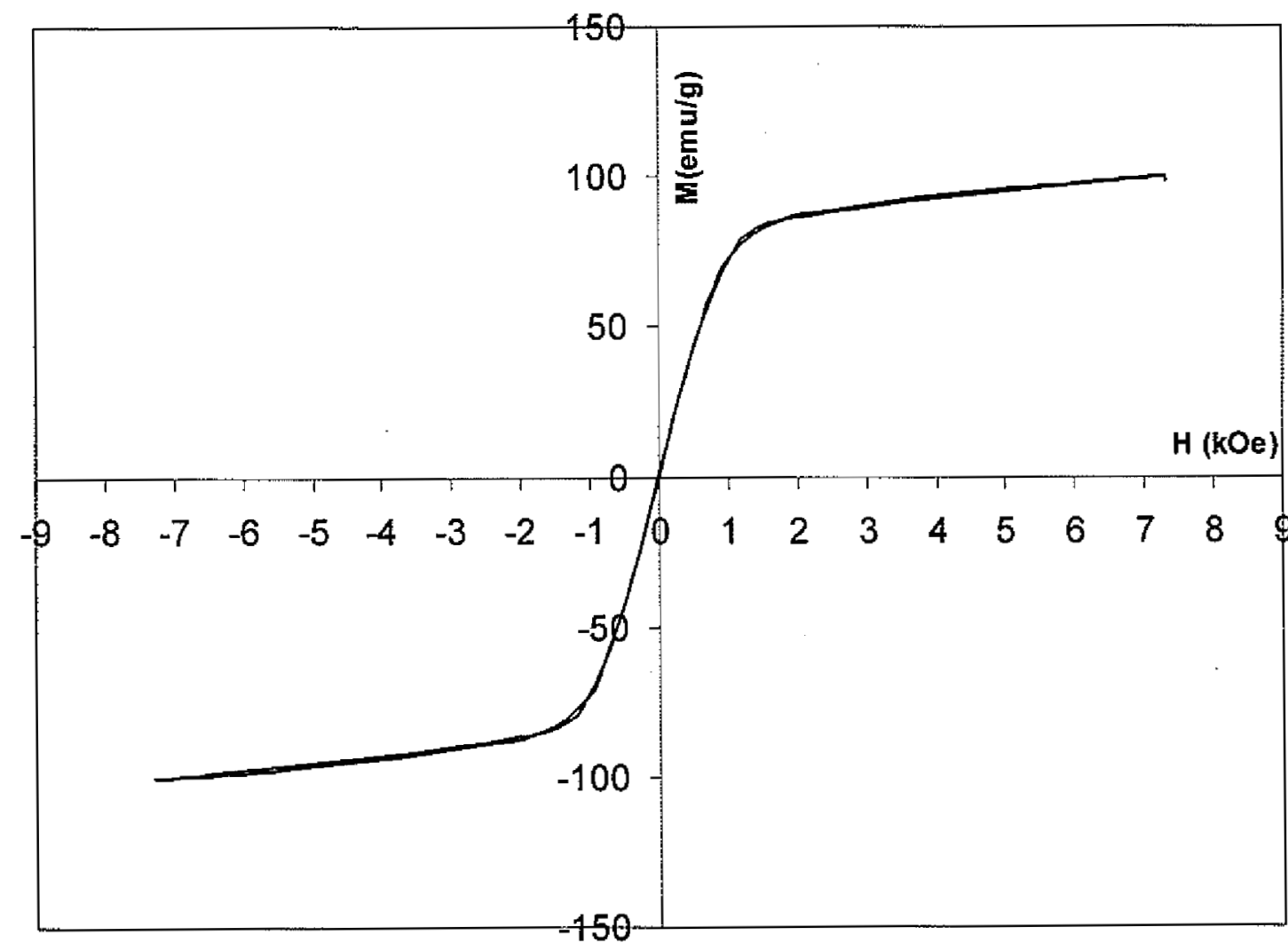

Fig.7- 54 VSM measurement for polycrystal sample $\mathrm{Gd}_{5}\left(\mathrm{Si}_{3} \mathrm{Ge}_{1}\right)$ at room temperature

The M. vs. H hysteresis loop measured by VSM method is shown in Fig.7-54. The result showed that $\mathrm{Gd}_{5}\left(\mathrm{Si}_{3} \mathrm{Ge}_{1}\right)$ is a soft magnetic material with a coercivity of 7 Oe and a saturation magnetization of $100 \mathrm{emu} / \mathrm{g}$ (about $0.74 \times 10^{6} \mathrm{~A} / \mathrm{m}$ ).

\section{Conclusions}

Thermal expansion measurement and magnetization versus temperature showed that $\mathrm{Gd}_{5}\left(\mathrm{Si}_{3} \mathrm{Ge}_{1}\right)$ has a second order transition, with a Curie temperature of $319 \mathrm{~K}$. 
Magnetostriction measurements showed a small value, and this suggests that giant magnetostriction only can be obtained in these materials when it is accompanied by a first order magnetocrystalline transition. VSM measurement shows it has a coercivity of $7 \mathrm{Oe}$. 


\section{Conclusions}

For clarity reason, the conclusions were made by regions and by sample examined.

\section{Region I: $0 \leq \mathrm{x} \leq \mathbf{0 . 3}$}

Magnetostriction and thermal expansion measurements were carried out on a polycrystal $\mathrm{Gd}_{5}\left(\mathrm{Si}_{0.15} \mathrm{Ge}_{3.85}\right)$ sample. When it was cooled from room temperature $(296 \mathrm{~K})$ down to $15 \mathrm{~K}$ with zero field, thermal expansion measurement shows that there is no abnormal strain change due to a second order transition ( from paramagnetic state (PM) to antiferromagnetic state (AFM) at high temperature region ) and a first order transition ( from AFM to ferromagnetic state (FM) at low temperature region). However, it is found that a magnetic field of $20 \mathrm{kOe}$ can induce a transition from AFM to FM at $15 \mathrm{~K}$. Subsequent magnetostriction measurements at $\Upsilon=15 \mathrm{~K}$ a giant magnetostriction about $1350 \mathrm{ppm}$ when $\mid \mathrm{H}_{\max }=20 \mathrm{kOe}$, and a transition found at $\mathrm{T}=25 \mathrm{~K}$ in heating curve under zero field is believed to be a transformation from $\mathbf{F M}$ to $\mathbf{A F M}$.

\section{Region II: Region II: $0.4 \leq x \leq 0.503$}

\section{(a) Single crystal $\mathbf{G d}_{5}\left(\mathbf{S i}_{1.95} \mathbf{G e}_{2.05}\right)$}

Measurements of thermal expansion of single crystal $\mathrm{Gd}_{5}\left(\mathrm{Si}_{1.95} \mathrm{Ge}_{2.05}\right)$ during cooling and heating were conducted for the first time. A very steep change in strain with temperature was observed when the material underwent a phase transformation. This was an unusual simultaneous magnetic and structural phase transformation from a ferromagnet with orthorhombic crystal structure below the transition temperature $T_{c}$ to a paramagnet with monoclinic crystal structure above $T c$. This transition temperature $T_{c}$ was found to depend on magnetic field, and to exhibit hysteresis with $T_{c}$ depending on whether the material was 
being cooled or heated. In the absence of a magnetic field $T_{0}$ is $267 \mathrm{~K}$ on cooling and $269 \mathrm{~K}$ on heating. However, when the material was subjected to a magnetic induction $\mathrm{B}$ in the range $0-2.5$ Tesla, the transition temperatures, on both cooling and heating, were found to increase linearly with temperature by about $4.9 \mathrm{~K} /$ Tesla. This rate of change of transition temperature with magnetic field was in good agreement with calculations based on the assumption that the additional energy due to the magnetic field can suppress the thermal vibration of $\mathrm{Gd}$ atoms and that the additional thermal energy per Gd atom needed to cause the phase transition to occur is equal to the additional magnetic energy of each $\mathrm{Gd}$ atom caused by the magnetic field.

\section{( b ) Single crystal sample $\mathrm{Gd}_{5}\left(\mathrm{Si}_{0.5} \mathrm{Ge}_{0.5}\right)_{4}$}

TE and MS were measured along the c-axis by the strain gauge method. The transition temperature was found to be $259.5 \mathrm{~K}$ on cooling, $261.5 \mathrm{~K}$ on heating. The abrupt change in strain and the temperature hysteresis indicate that it is a first order transition. MS measurements have been conducted at $15 \mathrm{~K}, 258 \mathrm{~K}$, and $265 \mathrm{~K}$. At $15 \mathrm{~K}$, the magnetostriction amplitude was only $3 \sim 4 \mathrm{ppm}$; at $258 \mathrm{~K}$, magnetostriction amplitude is about $100 \mathrm{ppm}$. At $265 \mathrm{~K}$, which is above the Curie temperature, a giant magnetostriction was found to be about $2000 \mathrm{ppm}$. This unusual behavior is due to the fact that the external magnetic field can increase the Curie temperature beyond $265 \mathrm{~K}$, resulting in a first order magnetocrystalline phase transition. The results reveal that the giant magnetostriction in $\mathrm{Gd}_{5}\left(\mathrm{Si}_{0.5} \mathrm{Ge}_{0.5}\right)_{4}$ only occurs as a result of the magnetic-structural transformation. 
Thermal expansion was also conducted along the $b$ axis of a single crystal sample $\mathrm{Gd}_{5}\left(\mathrm{Si}_{2} \mathrm{Ge}_{2}\right)$ under $0,0.5,1.0,1.5,2.0$ Tesla. It was found to be a first order magnetocrystalline transition, because a $3 \mathrm{~K}$ temperature hysteresis and a two phase coexistence were found in thermal expansion and isothermal magnetization process. It was also found that the dependence of transition temperatures on the applied magnetic field, on cooling, it is about $5.92 \mathrm{~K} / \mathrm{T}$; on heating it is $5.72 \mathrm{~K} / \mathrm{T}$. A magnetostriction of $770 \mathrm{ppm}$ was found at $\mathrm{T}=268 \mathrm{~K}$

Along the $\mathrm{a}, \mathrm{b}$ and $\mathrm{c}$ axes, magnetization measurements were conducted at $\mathrm{T}=15 \mathrm{~K}$ and 265 $\mathrm{K}$ by using a SQUID magnetometer. The results show that the easy axis is the $\mathrm{b}$ axis and coexistence of the ferromagnetic state and the paramagnetic state occurs at $\mathrm{T}=265 \mathrm{~K}$. Furthermore, a magnetic transition from the paramagnetic into the ferromagnetic state was also observed. The thermal expansion measurements under different magnetic field strengths showed a temperature hysteresis on cooling and on heating. This confirmed that it is a first order transition. It was found that an applied magnetic field can shift the transition temperature to higher temperatures, but with a different rate along different principal axes. Giant magnetostriction was observed only at temperatures higher than the transition temperatures found under zero field. It is believed that the giant magnetostriction is due to the magnetic-structural transition and the fact that this transition temperature is dependent on the applied magnetic field. At temperatures below the transition temperature, the magnetostriction values are small because they are due only to the spin-orbit coupling between $\mathrm{Gd}$ atoms, and are not caused by the structural transformation. 
These magnetostriction measurements show a series of anisotropic behaviors for single crystal sample $\mathrm{Gd}_{5}\left(\mathrm{Si}_{2} \mathrm{Ge}_{2}\right)$. It was found at temperatures above $\mathrm{T}_{\mathrm{c}}$ the magnetostriction was large and that the sign of the magnetostriction was different along different crystallographic axes. However for temperatures below $T_{c}$ the magnetostriction was small and the sign of the magnetostriction was same along different crystallographic axes.

\section{(c) Polycrystal sample $\mathbf{G d}_{5}\left(\mathbf{S i}_{2,99} \mathbf{G e}_{1,91}\right)$}

Two polycrystalline samples were made by using high purity $\mathrm{Gd}$ and commercial $\mathrm{Gd}$, respectively, but with $\mathrm{Si}$ and Ge starting materials of the same purity in both cases. Thermal expansion results showed that both samples exhibited a first order phase transformation, with a discontinuity in thermally-induced strain and with hysteresis in the Curie temperature. Magnetic force microscopy has been used to demonstrate the magnetic phase transformation process from paramagnetic to ferromagnetic upon cooling. It was found that the Curie temperature was lower and the thermally-induced strain higher, in the sample made from lower purity level Gd starting materials compared with the sample made from high purity Gd metal. These results indicate that the impurities (mainly $\mathrm{C}, \mathrm{O}, \mathrm{N}$ and $\mathrm{F}$ ) in the Gd starting material can significantly alter the strain and Curie temperature of $\mathrm{Gd}_{5}\left(\mathrm{Si}_{\mathbf{x}} \mathrm{Ge}_{1-\mathrm{x}}\right)_{4}$ alloys.

Magnetostriction and thermal expansion measurements were made on another polycrystalline sample of $\mathrm{Gd}_{5}\left(\mathrm{Si}_{2.09} \mathrm{Ge}_{1.91}\right)$, which was prepared by the induction melting with commercial grade Gd metal and high purity level $\mathrm{Si}$ and Ge starting materials. It was found that the transformation temperature is linearly dependent upon magnetic field of in the range $0-2$ Tesla. On cooling, the change rate is about $6.2 \mathrm{~K} /$ Tesla. On heating, it is about $6.6 \mathrm{~K} /$ Tesla. 
The transformation is classified as a first order type because it has a temperature hysteresis during a cooling and heating cycle. Magnetostriction measurements were conducted at $240 \mathrm{~K}$, $285 \mathrm{~K}, 297 \mathrm{~K}$; it was found that at $240 \mathrm{~K}$, the magnetostriction was the normal field induced one with a magnitude of $40 \mathrm{ppm}$. However, a giant magnetostriction about $-4500 \mathrm{ppm}$ was found at $285 \mathrm{~K}$. It was believed such a giant magnetostriction is due the unusual first order magneto-structural transformation.

\section{Region III: $0.575 \leq x \leq 1$}

\section{(a ) Single crystal $\mathrm{Gd}_{5}\left(\mathrm{Si}_{2,3} \mathrm{Ge}_{1.7}\right)$}

The $M$ vs. $T$ measurement showed that for $\mathrm{Gd}_{5}\left(\mathrm{Si}_{2.3} \mathrm{Ge}_{1.7}\right)$ there existed a second order transformation at $\mathrm{T}=307 \mathrm{~K}$ : below $307 \mathrm{~K}$ it was in a ferromagnetic state; above $307 \mathrm{~K}$ it was in a paramagnetic state. The $M$ vs. $H$ measurements showed that for this chemical composition the easy axis is the $c$ axis and the hard axis is the $b$ axis. These results also showed that it is a soft magnetic material with coercivity of 7 Oe. Thermal expansion measurement shows a strong anisotropy not only in the measurement above room temperature, but also in the below temperature measurement. Magnetostriction measurements at room temperature along $a, b, c$ axis shows a small value, but a strong anisotropic behavior. MFM images taken on $\mathrm{a}, \mathrm{b}$ and $\mathrm{c}$ plane demonstrated the domain structures are also strongly anisotropic.

\section{(b) Polycrystal sample $\mathrm{Gd}_{5}\left(\mathrm{Si}_{3} \mathrm{Ge}_{1}\right)$}

We conducted the first study of $\mathrm{Gd}_{5}\left(\mathrm{Si}_{3} \mathrm{Ge}_{1}\right)$ using thermal expansion and magnetostriction measurements. The results show that there exists a second order magnetic transition at $\mathrm{T}=$ 
$319 \mathrm{~K}:$ paramagnetic $\leftrightarrow$ ferromagnetic. Thermal expansion measurement from room temperature to $326 \mathrm{~K}$ showed an anomaly at $\mathrm{T}=320 \mathrm{~K}$, which was caused by this second order transition. There was no temperature hysteresis in magnetization vs. temperature between the cooling and heating curves, and this result confirmed the fact that it is a second order transition. Magnetostriction value was only $8 \mathrm{ppm}$ under a magnetic field of $28 \mathrm{kOe}$. VSM measurement showed it is a soft magnetic material with a coercivity of 7 Oe. 


\section{References}

[1] Song Desheng, Li Guodong, History of electromagnetism: observation and utilization of electrical and magnetic phenomenona Popular Press Publishers, Guang Xi, China, 1987.

[2] B. D. Cullity, Introduction to Magnetic Materials, Addison-Wesley, Reading, 1972.

[3] http://hyperphysics.phy-astr.gsu.edu/hbase/hframe.html.

[4] D. C. Jiles, Introduction to Magnetism and Magnetic Materials $2^{\text {nd }}$ edition, Chapman \& Hall, 1998.

[5] Du-Xing Chen, James A. Brug, IEEE Trans. Magn. Vol. 27, No. 4, July, 1991.

[6] Kittel, C. Revs. Mod. Phys., 21, 541, 1949.

[7] E.du Tremolet de Lacheisserie, D. Gignooux, M. Schlenker, Magnetism (I) Fundamentals, Kluwer Academic Publishers, 2002.

[8] P. Ehrenfest, Acad. Sci., Amesterdam Mitt. Kammerligh Onnes Inst., Leiden, 36, 153 Supl. $75 b, 1933$.

[9] Hae-Geon Lee, Chemical Thermodynamics for Metals and Materials, Imperial College Press, 1999.

[10] H. Eugene Stanley, Introduction to phase transitions and critical phenomena, Clarendon Press, Oxford, 1971.

[11] L. Morellon, J. Blasco, P. A. Algarabel, M. R. Ibarra, Phys. Rev. B. 2000, 62, 1022.

[12] K. A. Gschneidner, Jr., V. K. Pecharsky, D. Fort, Phys. Rev. Lett. 78, 4281, 1997.

[13] O. Tegus, E. Bruck, K. H. J. Buschow, F. R. de Boer, Nature, Vol, 415, 10, Jan., 2002.

[14] A.O. Pecharsky, K.A. Gschneidner Jr., V.K. Pecharsky, C.E. Schindler, J. Alloys \& Comps. 338, 126-135, 2002.

[15] V. K. Pecharsky, K. A. Gschneidner, Jr., J. Alloys and Compounds, 260, 98-106,1997.

[16] W. Choe, V. K. Pecharsky, V.G. Young, A. O. Pecharsky, K.A. Gschneidner, Jr., and G. J. Miller, Phys. Rev. Lett., 84, 4617, 2000.

[17] V.K. Pecharsky, K. A. Gschneidner Jr, Advanced Materials, 13, No.9, May 3, 683-686, 2001. 
[18] Kittel C. Introduction to Solid State Physics, pp114-115, $6^{\text {th }}$ edition, New York, Wiley, 1986.

[19] Levy R. A. Principles of Solid State Physics, pp141-144, New York, Academic, 1968.

[20] P. Mohazabi, F. Behroozi, Eur. J. Phys. 18, 237-240, 1997.

[21] F. C. Nix, W. Schockley, Rev. Mod. Phys. 10, 1, 1938

[22] C. Booth, J. S. Rowlinson, Trans. Faraday Soc., 51, 463, 1995.

[23] P. J. Wojtowciz, J. G. Kirkwood, J. Chem. Phys., 33, 1299, 1960.

[24] C. Tuijin, H. Bakker, Phys. Status Solidi (b) 155, 107, 1989.

[25] Robert C. O'Handley, Modern Magnetic Materials: Principles and Applications, John Wiley \& Sons, Inc., 2000.

[26] Kollie, T. G., Phys, Rev. B., 16, 4872, 1977.

[27] J. D. James, J. A. Spittle, S. G. Brown, and R. W. Evans, Meas. Sci. Technol., 12, R1R15, 2001.

[28] Soshin Chikazumi, Physics of Ferromagnetism, Clarendon Press, $2^{\text {nd }}$ edition, Oxford, 1997.

[29] M. Kornetzki, Z. Physik, 87, 560, 1933.

[30] E. Callen, H. B. Callen, Phys. Rev., 139, A455-A471, 1965.

[31] C. G. Shull, J. S. Smart, Phys. Rev., 1256, 76, 1949.

[32] Martin, Y., Wickramasinghe, H. K. Appl. Phys. Letts., 50, 1455, 1987.

[33] Binning, G., Quayte, C. and Gerbert, C. Phys., Rev. Letts., 56, 930, 1986.

[34] V. K. Pecharsky, A. P. Holm, K. A. Gschneidner, Jr., R. Rink, Massive magnetic fieldinduced structural transformation in $\mathrm{Gd}_{5} \mathrm{Ge}_{4}$ and the nature of the giant magnetocaloric effect. Phys Rev Lett 91: 197204/1-197204/4, 2003.

[35] E. M. Levin, A. O. Pecharsky, V. K. Pecharsky, and K. A. Gschneidner, Jr., Phys. Rev. B 63, 064426, 2001. 
[36] L. Morellon, P. A. Algarabel, M. R. Ibarra, J. Blasco, and B. Garcia-Landa, Phys. Rev. B 58, R14 721, 1998.

[37] L. Morellon, J. Blasco, P. A. Algarale, M. R. Ibarra, Phys. Rev. B, Vol. 62, Number 2, $1022,2000$.

[38] M. Han, D. C. Jiles, J. E. Snyder, T.A. Lograsso, D. L. Schlagel, IEEE Trans. on Magn., Vol.38, No. 5, 3252-3254, 2002.

[39] J. Leib, J. E. Snyder, C. C. H. Lo, J. A. Paulsen, P. Xi, and D. C. Jiles, Vol 91 (10) pp.8852-8854, May 15, 2002.

[40] M. Han, D. C. Jiles, J. E. Snyder, C. C. H. Lo, J. S. Leib, J. A. Paulsen, A. O. Pecharsky, 8486-8488, 93, J. Appl. Phys., 2003.

[41] M. Han, D. C. Jiles, J. E. Snyder, T. A. Lograsso, D. L. Schlagel, J. Appl. Phys. 95, No. 11, 6945-6947, 2004.

[42] A. K. Jena, M. C. Chaturvedi, Phase transformation in materials, Prentice Hall, 1992.

[43] D. C. Jiles, S. J. Lee, M. Han, C. C. H. Lo, J. S. Leib, J. A. Paulsen, A. O. Pecharsky Journal of Magnetics $8(1), 1-6,2003$.

[44] M. E. Fine, Introduction to phase transformations in condensed systems, Macmillan, New York, 1965.

[45] V. K. Pecharsky, K. A. Gschneidner, Jr., Magnetocaloric effect associated with magnetostructural transitions, MCE and Magnetostructural transformation, (upcoming publication), Springer, 2004.

[46] http:/www.vishay.com/brands/measurements_group/guide/index.htm

[47] J. Szade, G. Skorek, A. Winiarski, J. of Crystal Growth, 205, 289-93, 1995.

[48] J. S. Meyers, L. S. Chumbley, F. Laabs, A. O. Pecharsky, Determination of phases in as prepared $\mathrm{Gd}_{5}\left(\mathrm{Si}_{\mathrm{x}} \mathrm{Ge}_{1-\mathrm{x}}\right)_{4}$, where $\mathrm{x}=2$, accepted by Scripta Materialia for publication. Verhoeven, JD. Fundamentals of physical metallurgy, 2003. 


\section{Appendix A}

\section{Operation Procedures for Janis Closed Cycle Refrigerator}

These next steps should be performed with latex gloves on to reduce finger printing

\section{Opening the Cryostat}

1. Make sure the High-vacuum, roughing, and the foreline valves are closed

2. Open the vent valve to bring the cryostat to atmospheric pressure. Make sure the evacuation valve is also open

3. Disconnect the vacuum pump from the cryostat

a) Remove the tube clamp from the tube nearest to the vacuum pump

b) Note that there is rubber O-rings at these tube clamp connections

c) Remove the small tube clamp from at the other end of the flex tube.

4. Undo the two black latches holding the cryostat in place on the front side of the cryostat assembly

5. Tilt the entire assembly away from you. This will allow access to the vacuum chamber

6. Remove the tube clamp just below the helium pump.

7. Carefully slide off the outer cover

8. Care must be taken when removing the inner shroud.

9. Remove the 4 screws near the top of the inner shroud using an Allen wrench.

10. Carefully and slowly slide the inner shroud off.

\section{Closing the cryostat}

11. If a strain gauge is being used, check the resistance of the lead wire outside of the system before closing the cryostat. (Refer to page 10-12 for strain gauge information)

12. Wrap the lead wires in spiral fashion around the cold finger to minimize heat leaks.

13. Use the small plug to connect the wires together. Make sure that the plug doesn't contact the stage directly.

14. Carefully slide on the inner shroud

15. Be careful to not press the strain gauge leads or thermocouple leads into the inner shroud.

16. Fasten the shroud with the 4 screws.

17. Check the concentricity spacer at the bottom of the inner shroud to make sure that the screw is tight.

18. Put the outer shroud on rotating the shroud in a clockwise direction

19. Clamp the outer shroud in place with the tube clamp.

20. Connect the flexible tube to the vacuum pumping system using the tube clamps.

21. Make sure to keep the evacuation valve near the helium pump open.

22. Check the resistance between the strain gauge leads outside the system again.

\section{High vacuum pumping system}




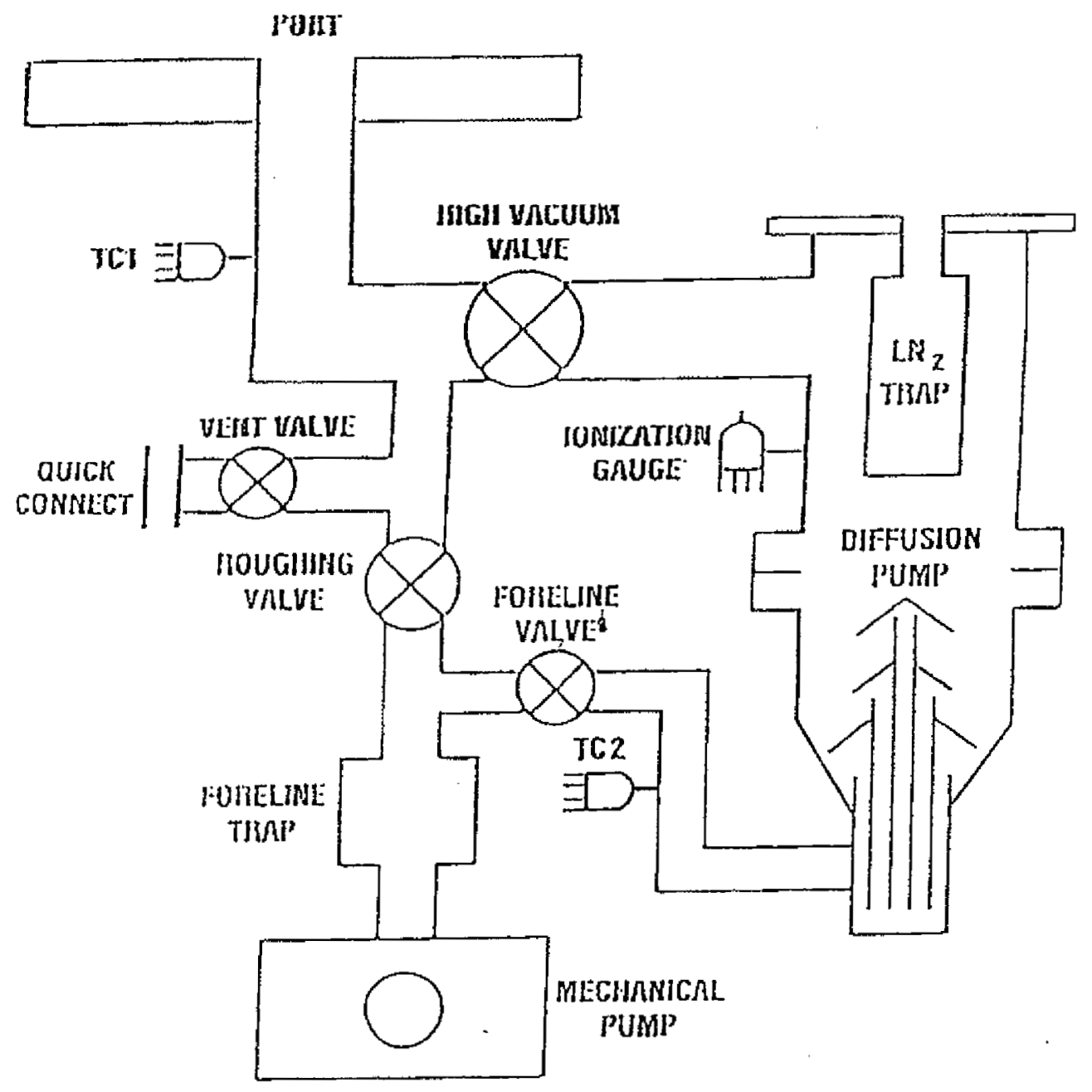

Fig. 1 


\section{Turn on the high vacuum pumping system}

A schematic diagram of the vacuum system can be seen on page 2

1. Close all valves

2. Turn on the main system switch

3. Turn on the mechanical pump. Make sure that the cooling fans are working. (Use your hand to feel the wind blowing)

4. Open the roughing valve.

5. Wait until the pressure in the system goes down to around $10^{-3}$ Torr (read from TC1)

6. Close the roughing valve and open the foreline valve.

7. After TC2 drops to about $10^{-2}$, turn on the diffusion pump.

8. Wait for about $20 \sim 30$ minutes for the oil in the diffusion pump to warm up sufficiently.

9. Wait for the pressure of the diffusion pump (read from TC2) to reach around 10-Torr.

10. Check the reading of TC1. If the pressure is higher than $10^{-2}$ Torr, close the foreline valve and open the roughing valve to pump it down to about $10^{-2}$ Torr. At the same time, keep an eye on TC2 and make sure that it is not higher than $10^{-2}$ Torr. You may need to switch between closing the foreline valve and opening the roughing valve and closing the roughing valve and opening the foreline valve.( Note: at the same time, do not turn off the diffusion pump)

11. When both $\mathrm{TC} 1$ and $\mathrm{TC} 2$ reach around $10^{-3} \mathrm{Torr}$ :

a) Close the roughing valve if not already closed

b) Open the foreline valve if not already open

12. Slowly open the high vacuum valve. (Mechanical Pump should be on too, foreline valve should be open)

13. Wait until TC1 \& TC2 are close to their lowest reading. (This may take $10 \mathrm{~min}$ )

14. Degas the filament of the Ionization gauge for $1 \sim 2$ minutes. Turn the degassing off.

15. Turn on the filament (Marked "FILAMENT") of the Ionization gauge and read the pressure in the close cycled system.

16. When the pressure goes down to the order of $10-6$ Torr, add liquid nitrogen to the cold trap located in the center of the top of the pumping cart.

NOTE: Do not put too much liquid nitrogen in; otherwise, it will take a long time for the nitrogen to evaporate. One liter may take about 9 hours to evaporate. Approximately $1 / 3$ to $1 / 2$ litters will work. More liquid nitrogen can be added if needed

17. For information on shutting down the compressor, refer to steps 6-12 in the next section -"Cooling Down the System (Turning on the compressor)"

\section{Cooling Down the System (Turning on the Compressor)}

The compressor can be turned on when the pressure in the system reaches the order of $10^{-7}$ Torr. It takes about 2.5 hours to reach $2 \times 10^{7}$ 
1. Turn on the temperature controller; write down the temperatures of Channel $A$ (which reads the Cernox sensor at the sample holder) and Channel B (which reads the silicon diode sensor at the refrigerator second stage)

2. On the operation $\log$ sheet, record

a) The ambient temperature (Channel A, B before starting operation)

b) The compressor pressure gauge (the helium pressure)

c) The cryostat system pressure (Ionization Gauge)

Static Helium pressure should be $245 \sim 255 \mathrm{psig}(1688 \sim 1757 \mathrm{kPa})$ at 70 to $80^{\circ} \mathrm{F}$

3. Open the valves of the water return and supply. There are 3 valves (ON THE RED PIPES) that need to be open. The sound of water running should be heard to verify the system is working

4. Turn on the compressor and record the following information in 15 minute intervals

a) The temperatures of Channel $A$

b) The temperatures of Channel $B$

c) The helium pressure

d) The vacuum of the system

Helium supply pressure should be 270 290 psig (1860 2000 kPa) at (50 to 100 \%)

5. Starting LabView Program, and compare the change of temperatures with time to the test result from Janis in table 1

6. When the temperature of Channel $A$ or $B$ is down to $80 \mathrm{~K}$, close the high vacuum valve. (Because the temperature of the liquid nitrogen in the cold trap is about $77 \mathrm{~K}$ )

Now the diffusion pump is not pumping the vacuum chamber of the system and the Ionization gauge is not reading the vacuum of the vacuum chamber. Only TCI is reading the pressure in the vacuum chamber.

7. Write down the pressure of the vacuum chamber when closing the high vacuum valve. NOTE: Keep an eye on the liquid nitrogen in the cold trap to make sure that there is some liquid nitrogen in the cold trap until the high vacuum valve has been closed. Otherwise, the pressure will increase to the order of 10-6.

8. Turn off the Ionization gauge. (Mark "Filament")

9. When all the liquid nitrogen in the cold trap is evaporated, close the foreline valve.

10. Turn off the diffusion pump and keep the mechanical pump running.

11. Wait at least 1 hour for the diffusion pump to cool down.

12. When the diffusion pump is cool. (The heater is near the bottom the diffusion pump). Turn off the mechanical pump.

13. The main switch can either be left on for pressure readings or turned off at this point.

14. Typically it takes about 4 hours to cool down from room temperature to $10 \mathrm{~K}$. 
Table 1: Test result of the close cycled system from Janis.

\begin{tabular}{l|c|c|l|l|c|c}
\hline Time & $\begin{array}{c}\text { Cernox } \\
\text { sensor }(\mathrm{K})\end{array}$ & $\begin{array}{c}\text { Silicon diode } \\
\text { sensor }(\mathrm{K})\end{array}$ & & Time & $\begin{array}{c}\text { Cernox } \\
\text { sensor }(\mathrm{K})\end{array}$ & $\begin{array}{c}\text { Silicon diode } \\
\text { sensor }(\mathrm{K})\end{array}$ \\
\hline 0 & 298.54 & 298.23 & & $2 \mathrm{~h} 15 \mathrm{~min}$ & 162.01 & 32.59 \\
\hline $15 \mathrm{~min}$ & 297.02 & 193.03 & & $2 \mathrm{~h} 30 \mathrm{~min}$ & 147.05 & 30.22 \\
\hline $30 \mathrm{~min}$ & 289.41 & 145.78 & & $2 \mathrm{~h} 45 \mathrm{~min}$ & 129.51 & 27.74 \\
\hline $45 \mathrm{~min}$ & 278.05 & 107.28 & & $3 \mathrm{~h}$ & 111.60 & 25.45 \\
\hline $1 \mathrm{~h}$ & 263.95 & 80.23 & & $3 \mathrm{~h} 15 \mathrm{~min}$ & 66.53 & 20.06 \\
\hline $1 \mathrm{~h} 15 \mathrm{~min}$ & 246.58 & 60.01 & & $3 \mathrm{~h} \mathrm{30 \textrm {min }}$ & 18.89 & 9.74 \\
\hline $1 \mathrm{~h} 30 \mathrm{~min}$ & 232.18 & 49.68 & & $3 \mathrm{~h} 45 \mathrm{~min}$ & 9.77 & 9.37 \\
\hline $1 \mathrm{~h} 45 \mathrm{~min}$ & 199.36 & 39.13 & & $4 \mathrm{~h}$ & 9.14 & 9.16 \\
\hline $2 \mathrm{~h}$ & 180.08 & 35.59 & & & & \\
\hline
\end{tabular}

\section{Warming up the Cryostat}

There are two procedures for warming the cryostat back up to room temperature. The first is for non-data acquisition and the second is for taking data during the warm up.

\section{Bring the cryostat back to room temperature without taking data}

1. Turn off the compressor. Keep the water running for a while to cool the compressor, and then close the water supply and return valves.

2. Warm the system up to room temperature. There are two ways to warm it up. One is to use the temperature controller and change the set point to room temperature, which is a faster way. The other is to leave the system to warm up by itself without the heater. (Read the next section for information on the temperature controller)

3. When the temperature of the system is around room temperature, STOP the CONTROL of the temperature controller if it was used to warm the system up.

4. The pressure in the system may go up to the order of $10^{-2} \sim 10^{-1}$ Torr. Close the foreline valve and open the roughing valve. Use the mechanical pump to pump the system down to around $10^{-3}$ Torr.

5. Turn off the temperature controller.

\section{Bring the cryostat back to room temperature while taking data}

This method requires use of the temperature controller.

\section{Temperature Controller for warming up at specific increments}

Channel A: Cernox sensor at the sample holder (4 325K) (to go to temperature higher than $325 \mathrm{~K}$, the Cernox sensor must be removed from the sample holder) 
Channel B: Silicon diode sensor at the refrigerator second stage (1.4 325K)

Channel C: Thermocouple at the high-temperature stage (no use in this system)

Channel D: Thermocouple at the sample holder (used mainly for measurement of temperatures higher than $325 \mathrm{~K}$ )

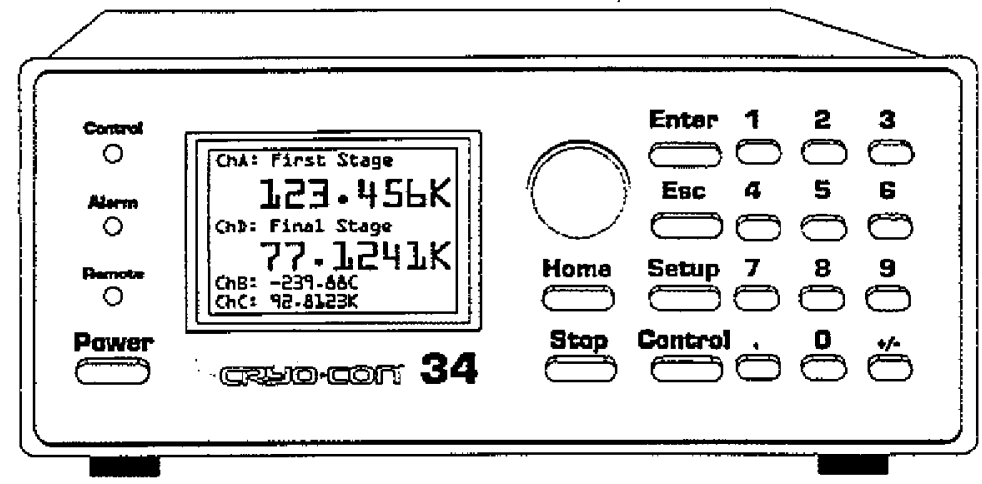

Fig. 2 Temperature controller (CRYO.CON ${ }^{\mathbb{B}}$ model 340)

\section{Easy operation of the heater temperature controller}

The heater controller is used to control and accelerate the warming up process.

NOTE: The heater should be used with the compressor still $\underline{\mathrm{ON}}$. This is because without the compressor on, the system will initially warm up too quickly.

1. The heater controller should already be on.

2. To get to the "Operate Display", press "Home".

This should be displaying the temp of channel $A$ and channel $B$ along with heater info.

\section{Change temperatures:}

1. Chose a control mode to use to reach the set point. (These modes are described below.)

a) From the Operate Display, press " 5 " to enter the "Heater Menu"

b) Rotate the knob to the lower left hand selection in the "heater menu".

c) Depress the knob and rotate it until the desired mode is displayed.

2. Change the set point.

a) From the Operate Display, press " 5 " to enter the "Heater Menu"

b) Rotate the knob until the curser is at the set point entry.

c) Enter the new value for the heater set point.

3. Press "Control" to start the heater and control the temperature. 
NOTE: A short cut to change the set point from the "Operate Display" is to press "Enter"

4. When the sample holder reaches room temperature, press the "stop" button.

5. Pressing, "stop" at anytime will stop the heater.

6. When testing is completed, turn off the compressor.

7. The cooling water should be left on for about 10 minutes to cool off the compressor

Wait for both "temp $A$ " and "temp $B$ " to reach $275 K$ before venting the system to prevent moisture entering the system and freezing.

8. Make sure the roughing, foreline, and high vacuum valves are closed and slowly open the vent valve to vent the system.

\section{Control Modes}

There are five control modes, i.e., Manual, PID, Table and RampP and RampT. "Table" is the one most likely to be used in this procedure however the user is free to use whichever works best for.

Table is most often used to reach a new set point and then stabilize at that temperature.

9. There are 2 PID tables stored in the temperature controller now.

10. PID Table 0 was set by Janis. Ranging from $12^{\circ} \mathrm{K}$ to $400^{\circ} \mathrm{K}$. It works in a wide range, but it cannot reach the lower temperature $\left(<150^{\circ} \mathrm{K}\right)$ set points exactly and get stable.

11. PID Table 1 was set by auto-tuning. Ranging from $12^{\circ} \mathrm{K}$ to $250^{\circ} \mathrm{K}$. At low temperatures it works better than PID Table 0 . PID at $400^{\circ} \mathrm{K}$ is copied from Table 1 to enlarge the range.

12. To read or change the parameters in the PID tables, from the Operate Display, press Setup to enter the Setup Menu, and then choose PID tables.

13. To change the Control Mode between PID Table 0 and PID Table 1, from the Operate Display, press " 5 " to go to the Heater Menu, choose Heater Configuration, and then change to the desired table.

14. Since it reaches the set point very quickly, wait for the sample to have the same temperature as the sample holder. It is recommended to increase the set point temperature slowly. Typically increase the set point temperature by no more than $5 \mathrm{~K}$ at any given time and wait for about 2 minutes for the temperature to stabilize before recording the strain at the set point temperature.

RampT is most often used to control the speed of changing the temperature.

15. In this control mode, the controller will approach a new set point at a user specified rate. When this set point is reached, the controller will revert to the PID Table control.

16. 16. To choose the rate and the PID table used, from the Operate Display, press 5 to go to the Heater Menu, choose Heater Configure. Here the ramp rate can be changed.

17. It is recommended to record the strain at the temperature interval set in the rate specified for the ramp. For example, if the set point is $150 \mathrm{~K}$, and you start from $100 \mathrm{~K}$, set the rate to be $+2 \mathrm{~K} /$ minute, then record the strain at the temperature of $100 \mathrm{~K}, 102 \mathrm{~K}$, $104 \mathrm{~K}, 106 \mathrm{~K}$, and so on at one-minute interval. 


\section{Auto Tuning}

Auto tuning is the process used to develop a PID table. This should not need to be done every time. Once a good functional PID table is created, another one should not be needed unless the system is changed in some way. It is recommended that if a new PID table is need, refer to the temperature controller manual and read pages $47-50$.

The two PID tables in the Temperature Controller:

00: PID table 0 (set by Janis)

\begin{tabular}{ccccc}
\hline Set point (K) & P & I & D & Power (W) \\
\hline 400 & 50 & 85 & 4 & 50 \\
\hline 250 & 40 & 80 & 3 & 50 \\
\hline 120 & 30 & 75 & 2 & 5.0 \\
\hline 40 & 25 & 60 & 2 & 0.5 \\
\hline 12 & 20 & 55 & 2 & 0.05 \\
\hline 0 & 0 & 0 & 0 & 0.05 \\
\hline
\end{tabular}

01: PID table 1 (set by auto tuning except the set point at 400K)

\begin{tabular}{cccccc}
\hline Set point (K) & $\mathbf{P}$ & I & D & Power (W) & $\begin{array}{c}\text { Power } \\
\text { output* }\end{array}$ \\
\hline 400 & 50 & 85 & 4 & 50 & \\
\hline 250 & 343 & 8 & 0 & 50 & \\
\hline 200 & 149 & 13.3 & 0 & 50 & \\
\hline 150 & 2184 & 4.18 & 0 & 50 & \\
\hline 120 & 71 & 19.9 & 0 & 5.0 & \\
\hline 100 & 127 & 16 & 0 & 5.0 & \\
\hline 70 & 4.43 & 35 & 0 & 5.0 & \\
\hline 50 & 3.31 & 27.1 & 0 & 5.0 & \\
\hline 35 & 1.29 & 21 & 0 & 0.5 & \\
\hline 30 & 0.99 & 18.3 & 0 & 0.5 & $83 \%$ \\
\hline 25 & 0.68 & 15.6 & 0 & 0.5 & $68 \%$ \\
\hline 14 & 0.29 & 8.98 & 0 & 0.5 & $31 \%$ \\
\hline 12 & 0.22 & 8.51 & 0 & 0.5 & $23 \%$ \\
\hline
\end{tabular}

* Power Output is the percentage of the heater power output when autotuning in Manual mode. It can be used as reference value for autotuning. 


\section{Analog Output on the Temperature Controller}

The "analog out" channel on the temperature controller can be used as a second control loop or as a scaled temperature output.

\section{For Temperature Output}

1. From the Operate Display, press "6" to reach the Analog Output Configuration Menu.

2 .To use it as a Scaled Temperature Output, scroll to the option on the lower right hand side of the menu and select the "Scale" setting.

3. Change the "P" value to the desired proportionality constant (see below for details)

4. Enter the value for the set point. (see below for details)

\section{Defining the Proportionality Constant and the Set point}

The analog output is scaled relative to the set point and the Proportional gain (P:) (They are different from the set point and the Proportional gain of the heater output), where the proportional gain is used as the scale factor. The analog output is displayed in percent. When the temperature is at the set point, the output is 50\%. The relation between the Proportional gain (P:) and the temperature range is shown in the table below:

\begin{tabular}{ccc}
\hline Proportional gain & $\begin{array}{c}\text { Temperature range displayed } \\
(0 \% \sim 100 \%)\end{array}$ & Total Range \\
\hline $\mathrm{P}=1$ & Set point $\pm 0.5^{\circ} \mathrm{K}$ & 1 \\
\hline $\mathrm{P}=0.1$ & Set point $\pm 5^{\circ} \mathrm{K}$ & 10 \\
\hline $\mathrm{P}=0.05$ & Set point $\pm 20^{\circ} \mathrm{K}$ & 40 \\
\hline $\mathrm{P}=0.01$ & Set point $\pm 50^{\circ} \mathrm{K}$ & 100 \\
\hline $\mathrm{P}=0.005$ & Set point $\pm 100^{\circ} \mathrm{K}$ & 200 \\
\hline $\mathrm{P}=0.002$ & Set point $\pm 250^{\circ} \mathrm{K}$ & 500 \\
\hline
\end{tabular}

For example, when the set point is $150^{\circ} \mathrm{K}$, and $\mathrm{P}=0.01$, then:

$$
\begin{aligned}
& \text { When } \mathrm{T}=100^{\circ} \mathrm{K} \text {, Output }=0 \% \text {; } \\
& \text { When } \mathrm{T}=120^{\circ} \mathrm{K} \text {, Output }=20 \% \text {; } \\
& \text { When } \mathrm{T}=150^{\circ} \mathrm{K} \text {, Output }=50 \% \text {; } \\
& \text { When } \mathrm{T}=199^{\circ} \mathrm{K} \text {, Output }=99 \% \text {; }
\end{aligned}
$$

The equation for finding a suitable value for $P$ is $1 /($ Total Desired Range $)=(P$ Value $)$

Typically, the Proportional gain of 0.01 with range of $100 \mathrm{~K}$ is used when high resolution is wanted, but you need to separate the whole range from about $10 \mathrm{~K}$ to $300 \mathrm{~K}$ into 3 parts.

Otherwise the proportional gain of 0.002 with range of $500 \mathrm{~K}$ is used to scan the whole range. 
NOTE: The current output of the Analog output is linear and $20 \mathrm{~mA}$ corresponds to $100 \%$. Resistors of different resistance can be used to convert the current into voltage. $(A$ $1 \%$ precision resistance of $100 \Omega$ is presently used) This gives us an output voltage of $0 \mathrm{~V}-2 \mathrm{~V}$ 


\section{Appendix B}

\section{Strain Gauge Installation Procedure}

Sample preparation for strain gauge measurements

1. Decide what type of strain gauge to be used.

2. Cut the sample to have two flat faces perpendicular to each other.

a) One is for sticking to the strain gauge, which should have an area a little larger than the gauge surface.

b) The other is for the bonding between the sample and the sample holder, which should be perpendicular to the strain direction to be measured. (As shown in the figure below)

3. Polish the two flat faces with abrasive paper, first with grit 400 then with grit 600 .

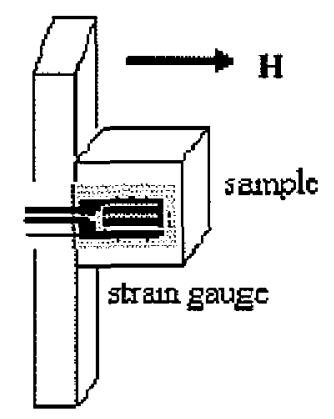

Fig. 3

Bonding strain gauges to samples

NOTE: This procedure should be performed while wearing latex gloves.

1 Polish surface or abrade surface of material with fine emery cloth (e.g. 600 grit).

2 Clean material surface with Micro-Measurements acid solution ( $\mathrm{HCl}$ ).

3 Neutralize surface with Micro-Measurements alkali solution.

4 Wipe surface with carbon isopropyl alcohol (or tetrachloride) to remove grease.

5 Lay gauge on "removable" scotch tape.

6 Put one end of tape down on sample and align gauge in desired direction.

7 When tape and gauge are correctly aligned, keeping one end of tape attached to the specimen, place a thin layer of adhesive on the surface of the gauge.

8 Run your finger along the tape beginning from the attached end to stick down the gauge and tape on to the specimen.

9 Cover the tape with non-adhesive material such as PTFE (Teflon) to apply continual pressure while adhesive sets.

10 Where necessary bake the sample and gauge or wait for about 24 hours to "cure" the bond. 


\section{Connecting the strain gauge to the lead wires}

We typically use a quarter bridge configurations. Three wires of different colors, i.e., white, red and black, are used for connecting the strain gauge to the pin by soldering

1. One lead wire is soldered to the two bare leads on one side of the gauge.

2. The other two lead wires are each connected to two separate leads on the other side of the gauge.

Remember the colors of the wires. The wires outside the system are connected to the wires inside by:

In

White

Red

Black
Out (Connecting to the Strain indicator)

White

Red

Brown

3. Wrap the bare wires with electrical-insulator such as Teflon tape.

4. Check the resistance between the wires outside the system to make sure that the electrical connection is correct and not shorted. (Typically it is $120 \Omega$ or $350 \Omega$.)

Bonding the sample to the sample holder

1. It is recommended to check the resistance between the wires outside the system before bonding.

2. To avoid stretching the strain gauge during the bonding, use removable tape to hold the sample near the sample holder.

3. Clean the surface of the sample holder and the face of the sample to be adhered to the sample holder, first with Acetone, then with Isopropanol.

4. Be careful not to get any chemicals on the Cernox sensor

5. GE7031 is used to bond the sample and the sample holder. Shake the bottle well before use.

6. Apply a very thin layer of adhesive to the sample holder (The wooden stick of a Q-tip can be used to do this)

7. Place the sample on it.

8. Hold the sample for a few minutes for it to stick to the sample holder.

9. Where necessary, non-adhesive materials such as removable tape or PTFE can be used to wrap the sample and sample holder together. (Remember to take the wrap off before closing the system)

10. To cure the bond faster, bake the sample and sample holder to no more than $52^{\circ} \mathrm{C}$, otherwise, wait for about 24 hours to cure the bond. 


\section{Appendix C}

\section{Strain Gauge Indicator (Model 3800)}

\section{Preliminary setup}

a) Set the POWER switch on.

b) Depress the AMP ZERO push button

c) Adjust the AMP ZERO potentiometer for a display reading of \pm 0000 .

d) Allow the unit to stabilize for a minimum of five minutes and repeat adjustment.

\section{Input connections}

Resistive strain gauges are normally connected at the binding posts on the front panel. These binding posts are color-coded in accordance with conventional practice and are clearly labeled. Fig4. shows input connections for quarter bridge configuration.
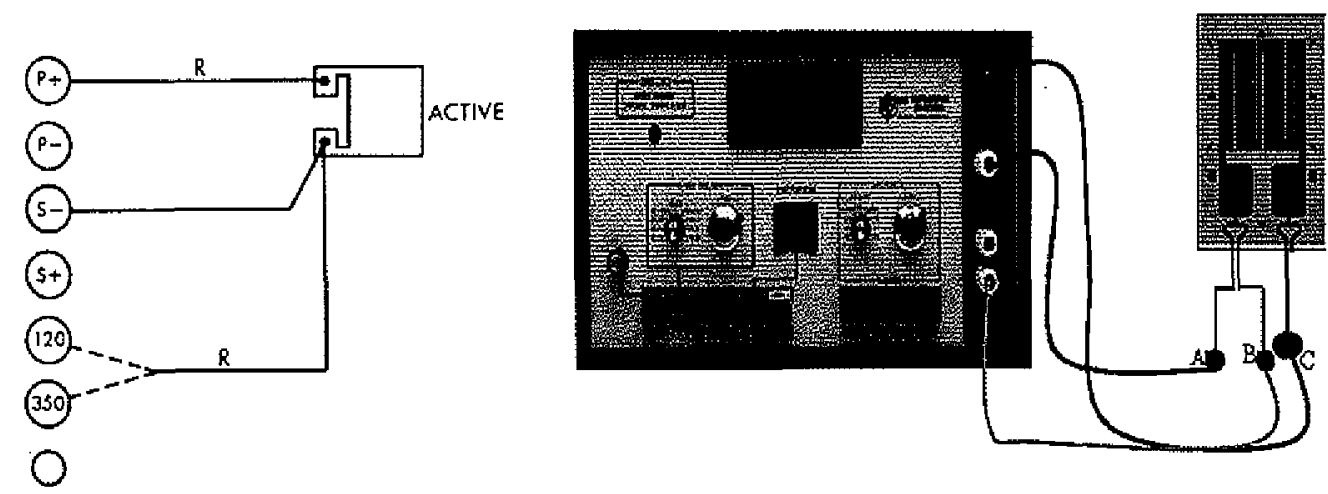

\section{Quarter Bridge}

Select the $1 / 4$ bridge configuration using the Full/ $1 / 2-1 / 4$ push button.

\section{Excitation}

Set the EXCITATION thumbwheel switch to the desired excitation voltage.

\section{Gauge Factor}

Depress the SET push button. Set the GAUGE FACTOR switch to the required range. Adjust the ten-turn Gauge FACTOR potentiometer for the exact gauge factor. (Gauge Factor is displayed by the LED readout when the SET push button is depressed).

\section{AMP ZERO}

It is desirable that AMP ZERO be adjusted at the gauge factor setting and the excitation voltage that will be used during a particular test sequence. Depress the AMP ZERO push button. Adjust the AMP ZERO potentiometer for a display of \pm 0000 .

Note: If EXCITATION of GAGE FACTOR will be varied during a test sequence, always adjust AMP ZERO at the lowest excitation voltage and the lowest gage factor. 


\section{RUN}

Depress the RUN push button. In this mode, the instrument is configured to read the strain as presented to the $\mathrm{S}+, \mathrm{S}$ - terminals.

\section{BALANCE}

Depress the $10 \%$ push button. Set the COARSE BALANCE switch to \pm 0 . Adjust FINE BALANCE potentiometer for a display of \pm 0000 .

If balance cannot be achieved, rotate the COARSE BALANCE switch to reduce reading to near zero. Complete balance with the FINE BALANCE potentiometer.

If balance still can not be achieved, depress the next higher BALANCE RANGE push button and repeat above step.

Note: if the GAGE FACTOR or EXCITATION will be changed during a particular test sequence, initial BALANCE adjustments should be made at the lowest gage factor and the lowest excitation voltage.

\section{CALIBRATION}

The model 3800 utilize the shunt calibration resistors across the internal $120 \Omega$ and 350 $\Omega$ dummy gages for quarter-bridge calibration. The calibration resistors simulate 5000 $\mu \varepsilon$ at a gage factor of 2.0. At gage factor other than 2.0, the displayed reading will be:

$$
5000 \times \frac{2.000}{G F}=\text { Calibration Number }
$$

When using the quarter-bridge configuration, the CAL position can be used in conjunction with the GAGE FACTOR potentiometer to compensate for lead wire resistance. This procedure is as followings:

a) Depress CAL push button

b) Calculate "Calibration Number"

c) Adjust the GAGE FACTOR potentiometer to give a reading equal to the calibration number.

d) Lock the GAGE FACTOR potentiometer in place. 


\section{Appendix D}

\section{Operation Procedure for VSM Measurement}

\section{Manual calibration procedure:}

1. Mount the standard Ni sample on the sample holder

2. Turn on the VSM controller to NORMAL.

3. Turn on the power supply. Turn the upper power supply on first before turning on the lower power supply. Ensure that the power supply has zero offset (zero output for zero input).

4. Check the Oersted range. Press "SWEP GENERATOR: SET PK FLD". Use the arrows to set it to $10 \mathrm{kOe}$.

5. Set the time constant by pressing "TIME CONSTANT". Set it to $\mathbf{1 0 0} \mathbf{m s e c}$.

6. Set the "SWEEP MODE" to FIELD.

7. Set the "SAMPLE DRIVE" to ON.

8. Press "STATIC". Use the arrows to set the applied field to the desired range, typically $10 \mathrm{kOe}$.

9. Use "DISPLAY SELECT" to change the display to EMU.

10. Use the arrows in the EMU sub-panel to select the correct display range.

11. Centering the sample:

a. Adjust the "Up-Down" screw to maximize the measured moment.

b. Adjust the "Front-Back" screw to maximize the measured moment.

a. Adjust the "Left-Right" screw to maximize the measured moment.

12. Adjust the "EMU CALIBRATE". Change the moment indication to the standard value.

13. Use "DISPLAY SELECT" to change the display to Oe.

14. Decrease the applied field by $1 \mathrm{Oe}$ at a time, waiting for it to settle, to $0 \mathrm{Oe}$ using arrows. (It takes about 20 seconds to settle)

15. Press the "STATIC" button.

16. Set the "SAMPLE DRIVE" to OFF.

17. Unmount the standard NiFe sample. 


\section{Manual sample set up procedure:}

1. Mount the specimen on the sample holder

2. Turn on the VSM controller to NORMAL.

3. Turn on the power supply. Turn the upper power supply on first before turning on the lower power supply. Ensure that the power supply has zero offset (zero output for zero input).

4. Check the Oersted range. Press "SWEEP GENERATOR: SET PK FLD". Use the arrows to set it to $10 \mathrm{kOe}$.

5. Set the time constant by pressing "TIME CONSTANT". Set it to $\mathbf{1 0 0} \mathbf{~ m s e c .}$

6. Set the "SWEEP MODE" to FIELD.

7. Set the "SAMPLE DRIVE" to ON.

8. Press "STATIC". Use the arrows to set the applied field to the desired range, typically $10 \mathrm{kOe}$.

9. Use "DISPLAY SELECT" to change the display to EMU.

10. Use the arrows in the EMU sub-panel to select the correct display range.

11. Centering the sample:

c. Adjust the "Up-Down" screw to maximize the measured moment.

d. Adjust the "Front-Back" screw to maximize the measured moment.

a. Adjust the "Left-Right" screw to maximize the measured moment.

12. Adjust the "EMU CALIBRATE". Change the moment indication to the standard value.

13. Use "DISPLAY SELECT" to change the display to Oe.

14. Decrease the applied field by $1 \mathrm{Oe}$ at a time, waiting for it to settle, to $0 \mathrm{Oe}$ using arrows. (It takes about 20 seconds to settle)

15. Press the "STATIC" button.

16. Set the "SAMPLE DRIVE" to OFF.

17. Unmount the sample. 
Sample set up procedure with the software:

1. Mount the specimen on the sample holder

2. Turn on the VSM controller to NORMAL.

3. Turn on the power supply. Turn the upper power supply on first before turning on the lower power supply. Ensure that the power supply has zero offset (zero output for zero input).

4. Check the Oersted range. Press "SWEEP GENERATOR: SET PK FLD". Use the arrows to set it to $10 \mathrm{kOe}$.

5. Set the time constant by pressing "TIME CONSTANT". Set it to $\mathbf{1 0 0} \mathbf{m s e c}$.

6. Set the "SWEEP MODE" to FIELD.

7. Open the Lake Shore Cryotronics, Inc. Software by clicking on the icon labeled "IDEAS VSM-Version 2"

8. Click "Ramp to", check "Field" in the pop up window, and enter a field.

9. Open the "MOMENT METER" window on the task bar and check to see if the units on the controller face and moment meter are the same. Change the time constant if necessary

10. Set the "HEAD DRIVE" to ON.

11. Centering the sample:

a. Adjust the "Up-Down" screw to maximize the measured moment.

b. Adjust the "Front-Back" screw to maximize the measured moment.

c. Adjust the "Left-Right" screw to maximize the measured moment.

12. Adjust the "EMU CALIBRATE". Change the moment indication to the standard value.

13. Use "DISPLAY SELECT" to change the display to Oe.

14. Click "Ramp to", check "Field" in the pop up window, and enter a 0 Oe field.

15. Once the field is back to zero, the sample is ready to run an experiment.

Running an experiment for hysteresis loops with software:

1. Click on "New Experiment"

2. Enter an experiment file name

3. Set the maximum field, number of points, experiment time, and the time constant; then click "OK"

4. Click "OK" on the "Experiment Box"

5. Enter the "Sample ID" and "File Name" (use same title)

6. Depending on the type of field being used change the Gaussmeter range on the VSM controller to cover the maximum field noted in the software.

e. i.e., if the max field is 50000 e use the $1000 \mathrm{kOe}$ setting on the VSM controller if the max field is 5000 e use the $300 \mathrm{kOe}$ setting on the VSM controller

7. Click "START".

8. When experiment is finished, turn off the "HEAD DRIVE" first

9. Save the experiment to your folder on the file server

10. Carefully remove the sample from the sample holder in the VSM

11. Place the sample back into it's box and the holder into it's container 


\section{Shutting down}

Shutting down the software:

1. Hold down alt, ctl, and del on the keyboard

2. click on the task manager

3. Click on the "Processes" tab

4. Select "ntvdm.exe" and click on "End Process"

5. Click "Abort" on the pop-up window for IDEAS-VSM

\section{Shutting down the system:}

1. Be sure that all materials are cleaned up

2. turn off the lower power supply

3. Turn off the upper power supply

4. Turn the VSM Controller key to the off position 


\section{List of Publications}

1. "Giant magnetostriction Behavior Around the Curie Temperature of the Single Crystal $\mathrm{Gd}_{5}\left(\mathrm{Si}_{0.5} \mathrm{Ge}_{0.5}\right)_{4}$ ", M. Han, D. C. Jiles, J. E. Snyder, T. A. Lograsso, D. L. Schlagel,6945-6947, Journal of Applied Physics, Vol.95, No. 11, June, 2004.

2. "Extraordinary Magnetomechanical Coupling as a Result of a Combined Magnetic Structural Transition in a New Class of Rare Earth Compound", D. C. Jiles, S. J. Lee, M. Han, C. C. H. Lo, J. E. Snyder, K. A. Gschneidner, V. K. Pecharsky, A. O. Pecharsky, T. Lograsso and D. Schlagel, Journal of Magnetics 8(1), 1-6, 2003.

3. "Thermal expansion studies on the unusual first order transition of $\mathrm{Gd}_{5}\left(\mathrm{Si}_{2.09} \mathrm{Ge}_{1.91}\right)$ : effect of purity of Gd", M. Han, D. C. Jiles, J. E. Snyder, C. C. H. Lo, J. S. Leib, J. A. Paulsen, A. O. Pecharsky, Journal of Applied Physics, 8486-8488, Vol. 93, Number 10, 15, May, 2003.

4. "Thermal expansion of single crystal $\mathrm{Gd}_{5}\left(\mathrm{Si}_{1.95} \mathrm{Ge}_{2.05}\right)$ showing unusual first order transformation", M. Han, J. A. Paulsen, J. E. Snyder, D. C. Jiles, T. A. Lograsso, and D. L. Schlagel, IEEE Transactions on Magnetics, 3252-3254, Vol. 38, No.5, September 2002.

5. "Unique Angular Dependence of the Unusual First Order Transition Temperature in $\mathrm{Gd}_{5}\left(\mathrm{Si}_{0.5} \mathrm{Ge}_{0.5}\right)_{4}$ ", M. Han, D. C. Jiles, S. J. Lee, J. E. Snyder, T. A. Lograsso, D. L. Schlagel, HD-02, International Magnetics Conference, March28-April 3, Boston, Massachusetts, USA, 2003.

6. "Thermal Expansion Studies on the Unusual First Order Transition of $\mathrm{Gd}_{5}\left(\mathrm{Si}_{2,09} \mathrm{Ge}_{1,91}\right)$ Made From the High Purity and Commercial Gd Metals", M. Han, D. C. Jiles, J. E. Snyder, C. C. Lo, J. A. Paulsen, GU-03, the 47th Annual Conference on Magnetism \& Magnetic Materials, Tampa, Florida, Nov. 2003.

7. "The Effect of Magnetic Field on the First Order Curie Point Transition of $\mathrm{Gd}_{5}\left(\mathrm{Si}_{0.5} \mathrm{Ge}_{0.5}\right)_{4}$ ", M. Han, D. C. Jiles, S. J. Lee, J. E. Snyder, T. A. Lograsso, and D. L. Schlagel. International Conference on Magnetism, Rome, Italy, July 27 -August 1 , 2003.

8. "The effect of magnetic field on the transition temperature along the a-axis of single crystal $\mathrm{Gd}_{5}\left(\mathrm{Si}_{1.95} \mathrm{Ge}_{2.05}\right)$ ", M. Han, D. C. Jiles, C. C. H. Lo, J.A. Paulsen, J. E .Snyder, American Physical Society, March Meeting, Indiana Convention Center; Indianapolis, Indiana, USA, 2002.

9. "Thermal Expansion Measurements Along a Axis for $\mathrm{Gd}_{5}\left(\mathrm{Si}_{1.95} \mathrm{Ge}_{2.05}\right)$ Showing a Unusual First Order Transformation", M. Han, J. A. Paulsen, D. C. Jiles, J. E. Snyder, T. A. 
Lograsso, D. L. Schlagel, DS-08, International Magnetics Conference, Amesterdam, the Netherlands, 2002.

10. "Microstructural and Magnetic Characterization of the Magnetic/Structural Transition in $\mathrm{Gd}_{5}\left(\mathrm{Si}_{\mathrm{X}} \mathrm{Ge}_{1-\mathrm{x}}\right)_{4}$ Materials", invited presentation, K. A. Gschneidner,Jr., V. K. Pecharsky, A. O. Pecharsky, G. Miller, D. C. Jiles, J. E. Snyder, S. J. Lee, M. Han, H. Tang, L. Lin, etc, TMS Fall Meeting, Columbus, Ohio, 2002.

11. "Giant magnetostriction Behavior Around the Curie Temperature of the Single Crystal $\mathrm{Gd}_{5}\left(\mathrm{Si}_{0.5} \mathrm{Ge}_{0.5}\right)_{4}$ ", M. Han, D. C. Jiles, J. E. Snyder, T. A. Lograsso, D. L. Schlagel, EC04, accepted for oral presentation in the 9th Joint Intermag/Magnetism and Magnetic Materials Conference, Anaheim, California, Jan., 2004

12. "Giant magnetostriction and thermal expansion in the germanium rich range of $\mathrm{Gd}_{5}\left(\mathrm{Si}_{\mathrm{x}} \mathrm{Ge}_{1-\mathrm{x}}\right)_{4}$ “, M. Han, D. C. Jiles, J. E. Snyder, A. O. Pecharsky, 2004 American Physical Society March Meeting, Montreal, Canada, March 22-26, 2004. 


\section{ACKNOWLEDGEMENTS}

This work was supported by the U.S. Department of Energy, Office of Basic Energy Science, Materials Science Division. The research was performed at Ames Laboratory. Ames Laboratory is operated for the US Department of Energy by Iowa State University under contract number W-7405-ENG-82.

I would like to express my sincerest appreciation to my major professor, Dr. David C. Jiles, who gave me such a wonderful opportunity to join the magnetic group in Ames Laboratory and opened such an exciting research area for me. I cannot be more grateful for all his guidance and encouragement throughout all my research work and study.

Ames Laboratory is well-known for its research on rare earth elements in the world, especially in recently years its leading research on room temperature magnetic refrigeration using the colossal magnetic caloric effect of $\mathrm{Gd}_{5}\left(\mathrm{Si}_{\mathrm{x}} \mathrm{Ge}_{1-\mathrm{x}}\right)_{4}$. I feel very lucky and proud to be one of the members in the Complex Materials Group, Materials \& Engineering Physics, I am also grateful for their financial support to my research.

I would like to thanks those people who have given me wonderful help: Dr. Chester. C. H. Lo, Dr. John. E. Snyder, Dr. Tom. A. Lograsso, Deborah Schlagel, Alexandra Pecharsky, Jason. A. Paulsen, Andy Ring, Fran Laabs. 
Finally, I am grateful for my lovely wife, Li Yuelin, for her encouragement, love, and sacrifice. Without her, I don't know whether I could go so far in this area. I am also indebted to my dear parents, brothers, and sister for their great love, hope on me. 